



\title{
STRUCTURAL AND BIOCHEMICAL INVESTIGATION OF tRNA MODIFYING ENZYMES
}

\section{Dissertation}

\author{
for award of the degree \\ Doctor rerum naturalium
}

by the Georg-August University Göttingen

within the graduate program

Biomolecules: Structure-Function-Dynamics

of the

Göttingen Graduate School for Neuroscience, Biophysics and Molecular Biosciences (GGNB)

by

Sven Johannsson

from Barsinghausen, Germany

Göttingen, 2018 



\section{Members of the Thesis Committee:}

Prof. Dr. Ralf Ficner (Reviewer)

Department for Molecular Structural Biology

Institute for Microbiology and Genetics

Georg-August-University Göttingen

Prof. Dr. Holger Stark (Reviewer)

Department of Structural Dynamics

Max-Planck-Institute for Biophysical Chemistry

Prof. Dr. Claudia Höbartner

Institute of Organic Chemistry

University of Würzburg

Members of the Extended Examination Board:

Prof. Dr. Henning Urlaub

Bioanalytical Mass Spectrometry Research Group

Max-Planck-Institute for Biophysical Chemistry

Prof. Dr. Kai Tittmann

Department of Molecular Enzymology

Schwann-Schleiden Research Centre

Georg-August-University Göttingen

Prof. Dr. Markus Bohnsack

Department of Molecular Biology

Centre for Biochemistry

Georg-August-University Göttingen 

I hereby declare that the PhD thesis "STRUCTURAL AND BIOCHEMICAL INVESTIGATION OF tRNA MODIFYING ENZYMES" is my own work that was prepared with no other sources and aids than quoted. This thesis, or parts thereof, have not been submitted elsewhere for any academic award or qualification.

Sven Johannsson

11th September 2018, Göttingen 



\section{PREFACE}

This doctoral thesis summarizes my work on the tRNA modifying enzymes tRNA guanine transglycosylase and the methyltransferase Dnmt2. The underlying work was carried out from August 2015 to September 2018 under supervision by Prof. Dr. Ralf Ficner at the GeorgAugust-University Göttingen and resulted in the following publications:

Sven Johannsson, Piotr Neumann, Alexander Wulf, Luisa M. Welp, Hans-Dieter Gerber, Matthias Krull, Ulf Diederichsen, Henning Urlaub and Ralf Ficner, Structural insights into the stimulation of $S$. pombe Dnmt2 catalytic efficiency by the tRNA nucleoside queuosine. 2018, Sci. Rep. 8, 8880 DOI: 10.1038/s41598-018-27118

Sven Johannsson, Piotr Neumann and Ralf Ficner, Crystal Structure of the Human tRNA Guanine Transglycosylase Catalytic Subunit QTRT1, 2018, Biomolecules, 8(3), 81 DOI: 10.3390/biom8030081

Parts of this thesis were presented at the following international conferences:

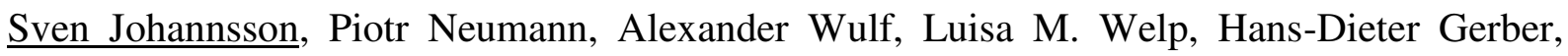
Matthias Krull, Ulf Diederichsen, Henning Urlaub and Ralf Ficner, Structural basis of increased Dnmt2 activity by queuine tRNA modification, $26^{\text {th }}$ Annual Meeting of the German Crystallographic Society (DGK), Essen, Germany, March 2018

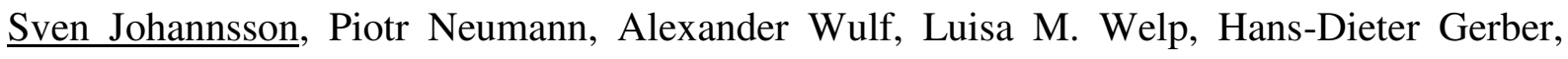
Matthias Krull, Ulf Diederichsen, Henning Urlaub and Ralf Ficner, Structural Basis for Dnmt2 Stimulation by Queuine tRNA Modification, $23^{\text {rd }}$ Annual Meeting of the RNA Society, Berkeley, USA, June 2018

Both first authored publications are included as part of this thesis, although not in chronological but topical order. The individual contribution of each author is stated at the beginning of the respective chapter. 


\section{CONTENTS}

Abstract

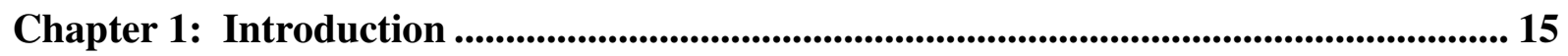

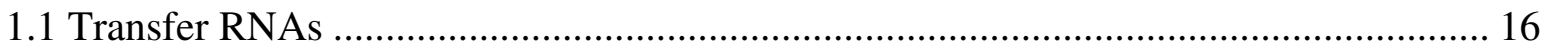

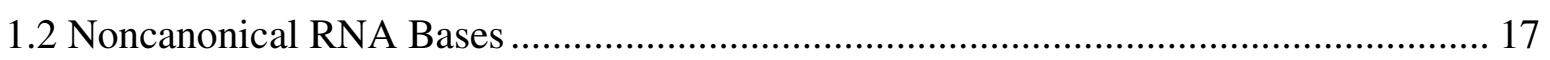

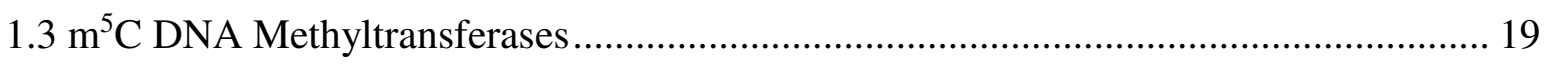

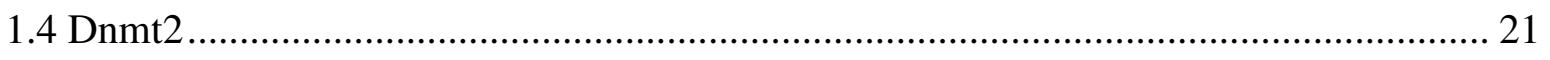

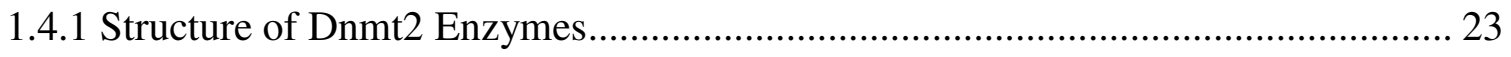

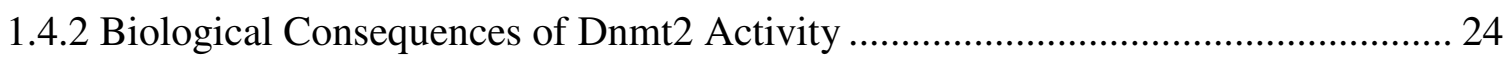

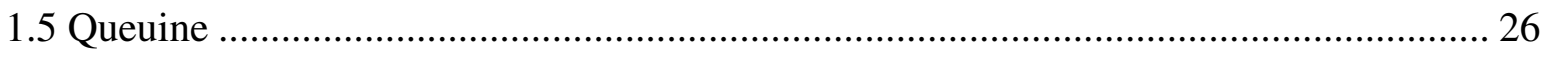

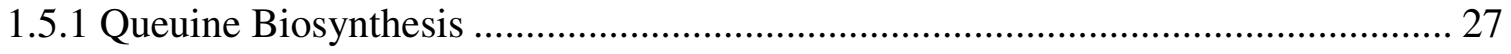

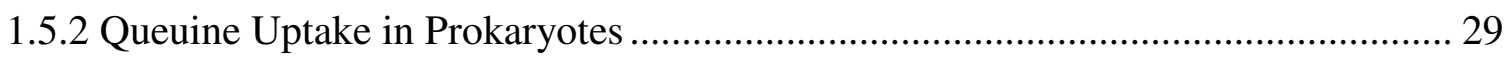

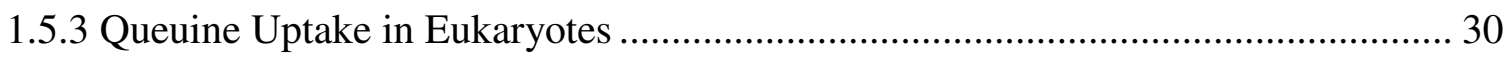

1.5.4 Further Modification of the Queuine Base .......................................................... 31

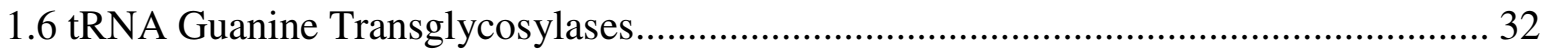

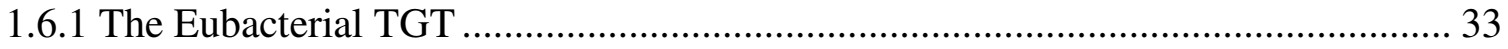

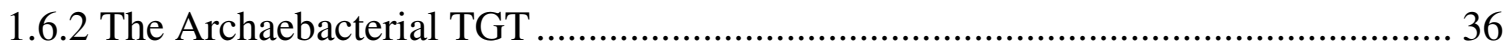

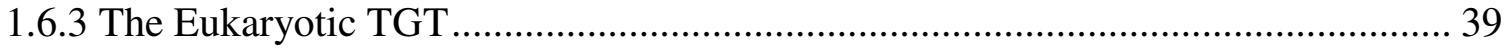

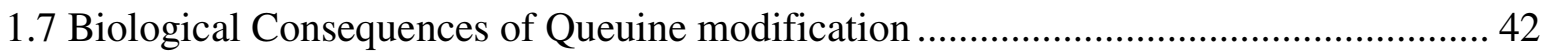

1.7.1 Modulation of Dnmt2 Dependent C38 Methylation............................................. 42

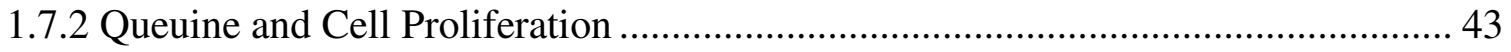

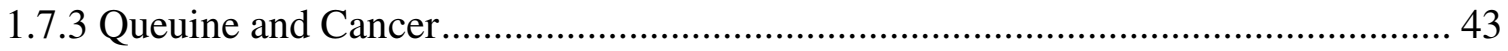

Chapter 2: Structural Analysis of QTRT1 ................................................................................. 45

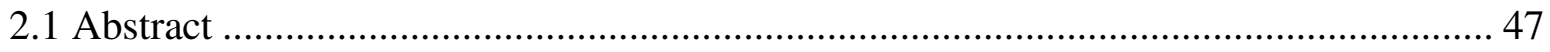

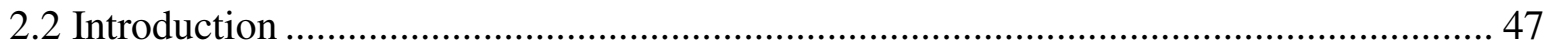

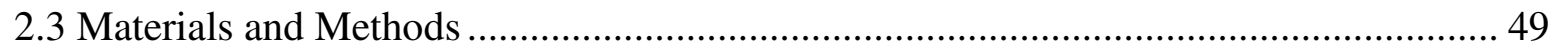

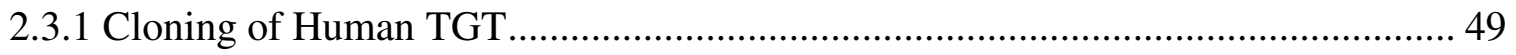

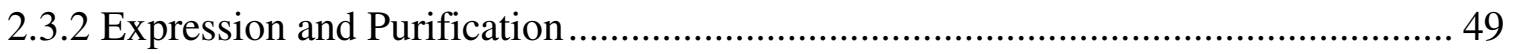

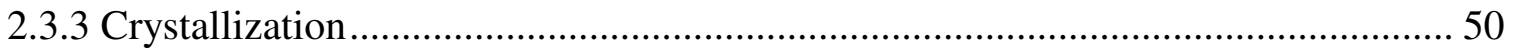

2.3.4 Data Collection, Molecular Replacement and Refinement ................................... 50

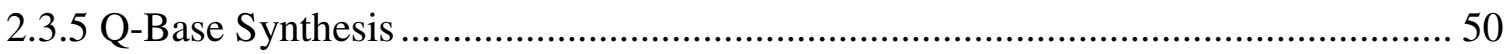

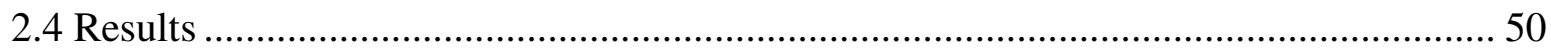


2.5 Discussion 54

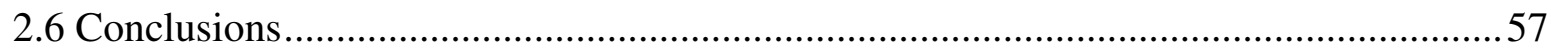

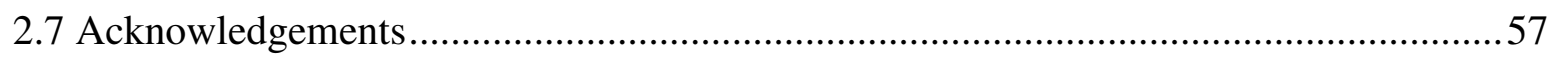

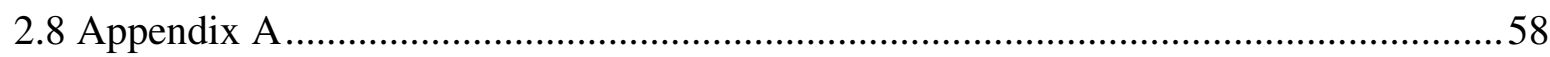

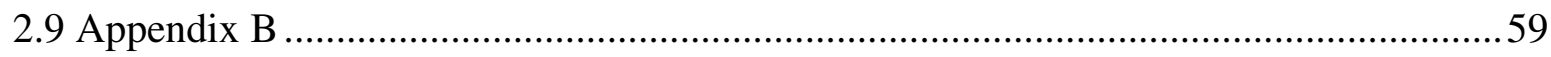

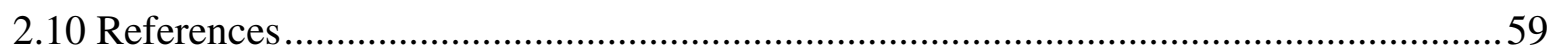

Chapter 3: Insights into Dnmt2 Stimulation by Queuosine ................................................63

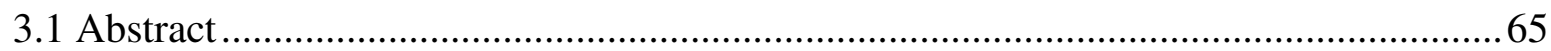

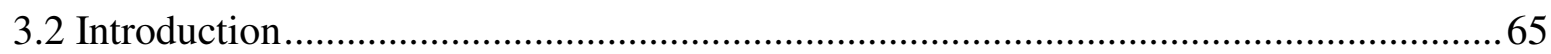

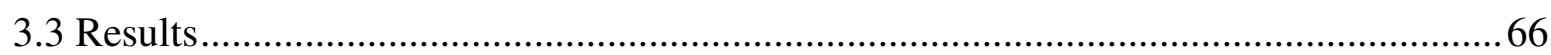

3.3.1 Methyltransferase Efficiency is Stimulated by Q34 in tRNA ${ }^{\text {Asp }}$...............................66

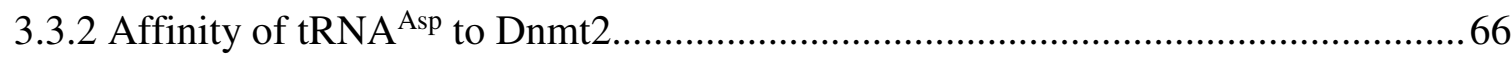

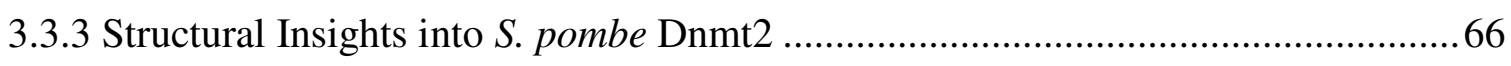

3.3.4 Analysis of the Dnmt2-tRNA ${ }^{\text {Asp }}$ Complex with UV Induced Cross-Linking ..........67

3.3.5 Dnmt2-tRNA Docking Places G34 in Close Proximity to the Active Site...............68

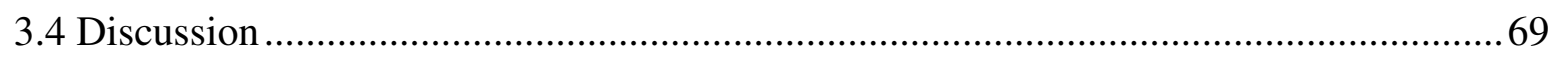

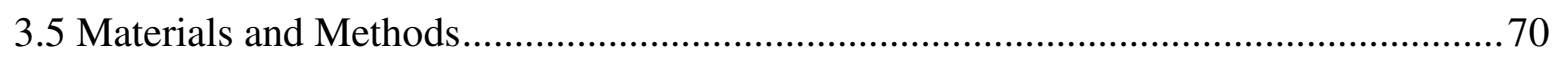

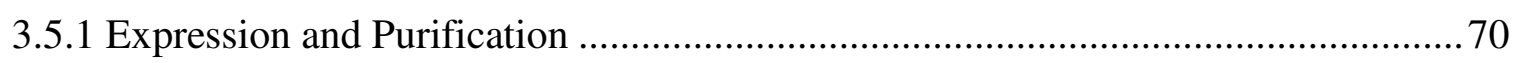

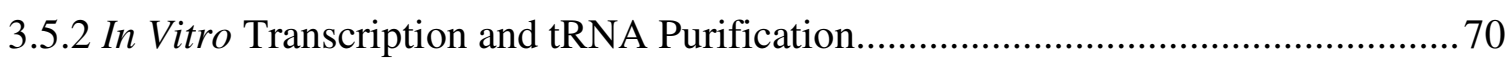

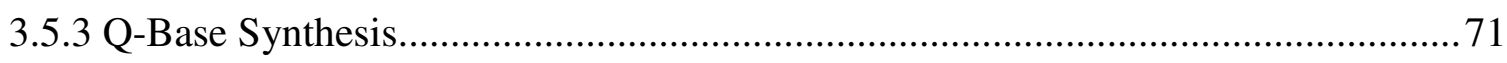

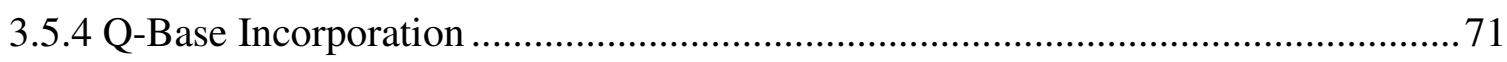

3.5.5 tRNA Labelling and Affinity Measurements .................................................... 71

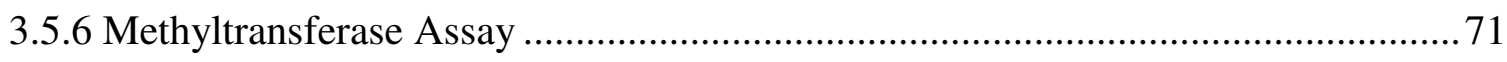

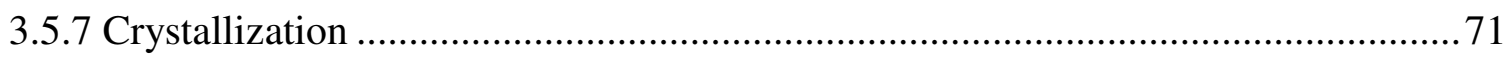

3.5.8 Data Collection, Molecular Replacement and Refinement .................................... 71

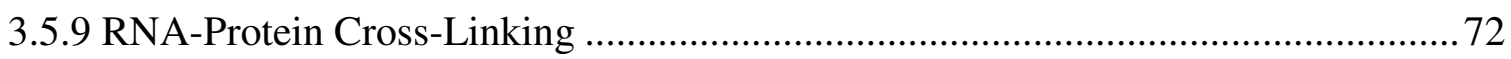

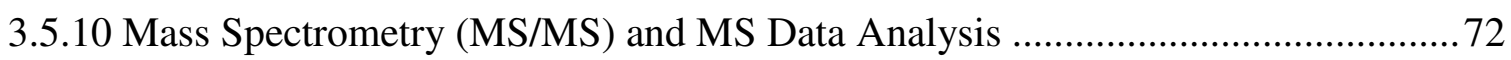

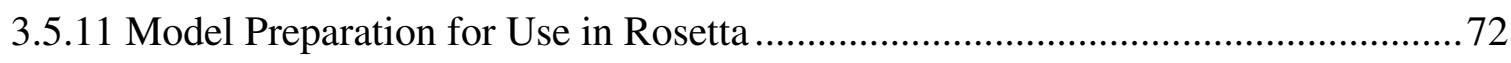

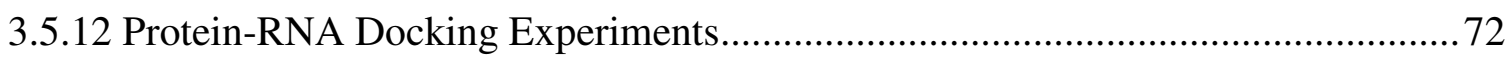

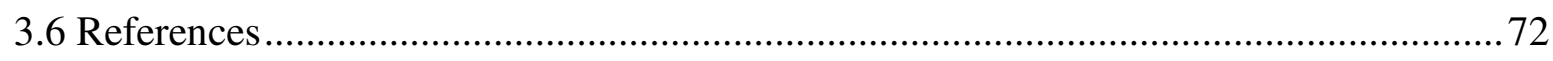

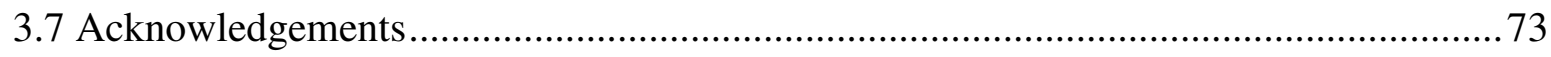

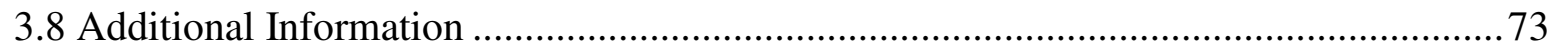

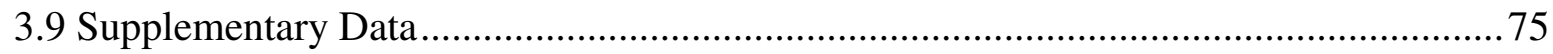


Chapter 4: Discussion .......................................................................................................................... 95

4.1 Investigation of RNA-Interacting Residues in QTRT1 ................................................. 96

4.2 Structural Investigation of QTRT1 Phosphorylation ................................................. 98

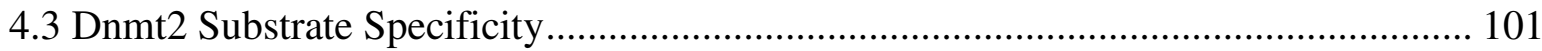

4.4 Impact of the S. pombe Dnmt2 Crystal Structure.................................................... 102

4.5 Biological Significance of Dnmt2 Complex Models ................................................... 103

4.6 How Queuosine Might Trigger Dnmt2 Activity ....................................................... 107

4.7 Discussion of a Dnmt2 Working Model................................................................. 108

Chapter 5: Synopsis and Outlook........................................................................................ 110

Chapter 6: References .................................................................................................................. 113

Abbreviations ........................................................................................................................................ 126

Acknowledgements............................................................................................................................. 129

Curriculum Vitae ................................................................................................................................. 131 


\section{ABSTRACT}

Posttranslational and posttranscriptional modifications have long been known to modulate and extend the properties of proteins and nucleic acids. A vast variety of non-canonical bases have been found on RNA molecules, with a huge number of them occurring on transfer RNAs (tRNAs).Here they modulate the molecule's stability, chemical properties or even its shape, which are crucial elements for the correct function of tRNAs. In contrast to modifications that have been described on proteins like histones, where a close interplay of modifications has been elucidated, least is known about how RNA modifications affect each other and whether they may be linked. Considering an average of 14 modifications occurring on an tRNA molecule simultaneously, the study of an eventual link between these modifications becomes imminent. In contrast to the numerous studies focusing on a single modification, recent publications have presented a link between the 7deaza-guanosine derivative queuosine $(\mathrm{Q})$, which occurs on the tRNA $^{\text {Asp }}$ wobble base 34 (Q34), and Dnmt2 mediated methylation of the C38 base $\left(\mathrm{m}^{5} \mathrm{C} 38\right)$ in a downstream manner. While investigation of this linkage identified C38 methylation to strongly depend on presence of queuosine in the tRNA target, least in known about the underlying molecular mechanism of this interplay.

Prior to this thesis, the structure of the tRNA guanine transglycosylase (TGT), which establishes Q34 modification by incorporating the modified queuine base into the tRNAs tRNA ${ }^{\text {Asp }}$, tRNA $^{\text {Asn }}$, tRNA ${ }^{\text {His }}$ and $\mathrm{tRNA}^{\mathrm{Tyr}}$, was unknown. Within this work the first structure of the catalytic subunit QTRT1 of the TGT heterodimer is reported. Investigation of this structure of the human QTRT1 reveals a high conservation, suggesting the reaction mechanism to be conserved from bacteria to men. Furthermore, the QTRT1 structure was solved in complex with the queuine base providing first insights into the accommodation of this hypermodified base. Within this thesis, the newly solved QTRT1 crystal structure is further investigated with focus on RNA interaction and phosphorylation, latter of which is implicated in TGT activity.

In a second part, the Dnmt2 methyltransferase is investigated biochemically and structurally with focus on Q34 substrate modification, as the dependence of tRNA ${ }^{\text {Asp }}$ C38 methylation in $S$. pombe has been shown to depend on the presence of TGT reaction product queuosine as part of the substrate in vivo. However, the underlying mechanism how queuosine modulates the activity of the $\mathrm{m}^{5} \mathrm{C} 38$ depositing enzyme Dnmt 2 is not known. As part of this work, queuine modification of tRNA ${ }^{\text {Asp }}$ alone is found to be sufficient to trigger Dnmt2 activity in vitro. 
Furthermore, a model of Dnmt2 tRNA substrate complex was generated by computational docking of the tRNA to the newly solved S. pombe Dnmt 2 crystal structure. Combination of biochemical and structural data lead to the conclusion that triggering of Dnmt2 activity by Q34 is mediated by optimal positioning of the relevant reaction components.

Substrate specificity of Dnmt2 is further investigated and set into relation with the $S$. pombe Dnmt 2 structure as well as previously deposited Dnmt2 structures. Furthermore, the docked Dnmt2 tRNA complex is found to be in high agreement with cross link data and identified as the most advanced model of Dnmt2 substrate interaction. Finally, a working model for Dnmt2 mediated methylation is proposed by combining data reported as part of this work with the other reported studies on the Dnmt2 enzyme. 


\section{CHAPTER 1: INTRODUCTION}

The discovery of deoxyribonucleic acid (DNA) molecules over a century ago (1), marked the first encounter of what is now understood as one most important discoveries in life sciences ever made: The genetic code. DNA consists of only four canonical nucleosides: The nucleosides comprising a purine base deoxycytidine $(\mathrm{dC})$ and deoxythymidine $(\mathrm{dT})$, and the pyrimidine base containing nucleosides deoxyguanosine $(\mathrm{dG})$, deoxyadenosine $(\mathrm{dA})$, with the corresponding ribonucleosides $\mathrm{C}, \mathrm{U}, \mathrm{G}$ and $\mathrm{A}$ in ribonucleic acids (RNAs). Followed by groundbreaking discoveries about its components and its architecture DNA and RNA are generally renowned as the basic building blocks of life.

The basic building blocks of DNA and RNA are the nucleic bases, which are covalently linked to a (deoxy-) ribose molecule. Linked by a phosphate group between the 5' position of one (deoxy-) ribose and the $3^{\prime}$ of a neighboring ribose molecule, multiple nucleosides are capable to form huge polymers that can store the genetic information through the specific sequence of the bases. To preserve the genetic information this polymer is stabilized by contact formation with an antisense strand, mediated by the bases, with consequent formation of the renowned double helix architecture $(2,3)$. In this helix base contacts are mediated by hydrogen bond formation between the purine bases and the pyrimidine bases, which allow biomolecules to "read" the genetic information and replicate it or transcribe the sequence into RNA. In RNA molecules the same specific base paring rules apply with $\mathrm{G} / \mathrm{C}$ and $\mathrm{A} / \mathrm{U}$. However, different types RNA molecules exist that do exhibit distinct functions apart from serving as a coding template for protein synthesis, as it is the case for messenger RNA (mRNA). Certain RNAs are capable to catalyze (bio-) chemical reactions $(4,5)$, while micro RNAs, small nuclear RNAs (snRNAs) and small nucleolar RNAs (snoRNAs) have been associated with gene expression, mRNA splicing and RNA modification respectively (6-8). One of the most abundant RNA molecules in the cell are transfer RNAs (tRNAs). These RNAs an essential tool to translate mRNA sequences into protein and therefore are featured by organisms belonging to all kingdoms of life (9). 


\section{$\underline{1.1 \text { TRANSFER RNAS }}$}

Transfer RNAs (tRNAs) were discovered more than 50 years ago and their discovery was vital for the understanding of the central biochemical dogma that DNA is transcribed into RNA which subsequently is translated into protein. With several million molecules, tRNAs are the most abundant RNA molecules in the cell (10). More than 400 tRNA genes are annotated in the human genome, with several of them being encoded not by one but multiple genes (11). Despite their high sequence diversity, tRNAs possess a similar secondary structure which is commonly referred to as the "cloverleaf", based on an analogy of both shapes. This tRNA fold is build up by five parts (Figure 1), the acceptor stem, that comprises the 5'- and 3'- end of the molecule, the D-arm, the anticodon stem and loop, which includes the name giving anticodon triplet, and the $\mathrm{T} \psi \mathrm{C}$-arm or $\mathrm{T}$-arm, named after the thymidine modification that was observed in this region. Part of every tRNA is also the CCA triplet, which is enzymatically linked to the 3'end of all tRNAs by the CCA-adding enzyme after their transcription (12-14). This sequence, consisting of only three nucleotides, is of imminent importance for aminoacylation, positioning of the tRNA in the ribosome and transcription termination (15-17).

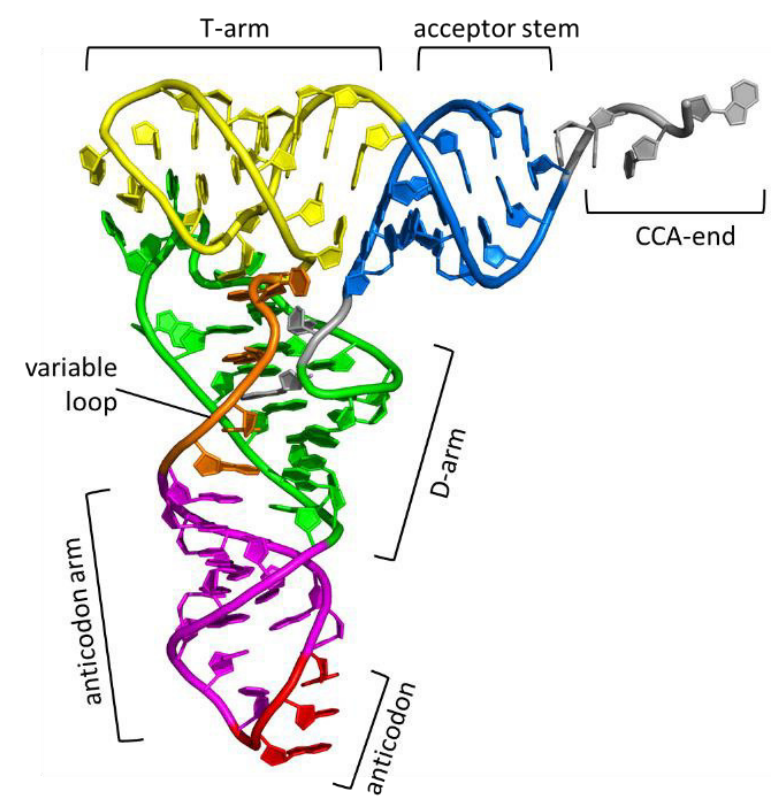

Figure 1. Structural organization of L-shaped tRNAs

Crystal structure of yeast tRNA ${ }^{\text {Phe }}$ (PDB-ID: 1EHZ) depicted as cartoon with individual representation of the four canonical bases. The individual parts comprised by the canonical tRNA fold are colored individually. 
In contrast to the canonical shape exhibited by most tRNAs, alterations in the structure have been observed for some tRNAs. They occur mainly in tRNAs from mitochondria, such as the mitochondrial tRNAs tRNA ${ }^{\text {Asp }}$ from human and the bovine tRNA ${ }^{\text {Phe }}$. In these tRNAs a highly altered nucleotide content and altered sizes of the D- and T-loop lead to an impaired interaction between these regions that is usually of importance for the global stability of tRNAs $(18,19)$. Even more drastic changes have been observed in tRNAs from nematodes, missing entire elements of the canonical tRNA structure like the D-loop and/or the T-loop, which in consequence adopt a fold very distinct from the common cloverleaf $(20,21)$. Interestingly, several studies indicate that these non-canonical tRNAs may still be fully functional in protein translation. However, these structural specialties are restricted to individual tRNAs and the Lshape is typical for the vast majority of tRNAs $(12,22)$.

After maturation, the biological purpose of tRNAs is mediated by multiple protein interactions upon which the tRNA structure might undergo conformational changes (23). Interaction with aminoacyl-tRNA-synthases (aaRS), who charge the tRNA with the corresponding amino acid at the CCA end, is mainly localized at the anticodon loop and the CCA end, which serve as recognition- and as target site for the aaRS respectively $(24,25)$. AaRS tRNA complex structures revealed that the tRNAs do adopt a different fold at these interacting regions upon binding that allows the modifying enzyme to specifically recognize its substrate and perform the enzymatic transfer reaction $(26,27)$, thus highlighting the flexibility of the tRNA fold $(23)$.

\subsection{NONCANONICAL RNA BASES}

To date, only a single digit number of modifications on DNA are known. These are mainly limited to methylation of the bases cytosine, adenine and uracil (28). In contrast to this low number, at least 171 different modifications are present on RNAs (29) adding a high variety to the chemical properties of the canonic RNA bases. Alterations of the RNA's canonical structure mainly involve the bases themselves and are found to occur on all four nucleosides. The nature of these modifications is highly diverse, ranging from rather simple modifications, such as methylation or acetylation, to the addition of complex structures, so called hypermodifications, which can even involve changes of the base ring system (28).

With a total of 93 modifications, tRNAs are one of the most heavily modified RNA species (29, 30). The composition of these modifications as well as their abundance differs in the three 
kingdoms of life $(28,31)$, with $6.6 \%$ of all tRNA bases carrying modifications in prokaryotes and a generally higher abundance of non-canonical nucleotides in eukaryotes where an average of $16.6 \%$ of all bases are modified (32). Some of the modifications, such as pseudouridine $(\psi)$, are observed on most tRNAs and generally more abundant while other, usually more elaborate structures like queuine (Q) or isoprenyl rests, are typically found only on a tRNA subset (28, 33).

The consequences of tRNA modifications are as diverse as their chemical properties, ranging from effects on stability over induction of structural alterations to fine tuning of interactions (34-36). A prominent example how a small modification can be important for a global tRNA structure is the methylation of the A9 base in the mitochondrial tRNA ${ }^{\text {Lys }}$. In vitro transcription of the respective RNA sequence results in a misfolded tRNA, which is highly diverse from the canonical cloverleaf structure of tRNAs. The introduction of a methyl group at position 1 of the A9 base $\left(\mathrm{m}^{1} \mathrm{~A} 9\right)$ results in the disruption of the watson-crick base paring with U64 and consequently results in the formation of the classical tRNA topology $(35,37)$. Beside supporting the tertiary structure, it has been shown that tRNA modifications have the potential to increase the stability of the respective RNA molecule (31). Thermophilic organisms rely on increased stability of their cellular components to heat. To meet this requirement, thermophiles do utilize a high diversity of modifications, like pseudouridine $(38,39)$. Presence of this modified base at position 39 of the tRNA ${ }^{\text {Lys }}$ anticodon stem stabilizes the structure through improved stacking of the bases and stronger base paring, resulting in a $5{ }^{\circ} \mathrm{C}$ higher melting temperature (40).

A similar effect has been observed as a consequence of archaeosine $\left(\mathrm{G}^{*}\right)$ modification at position G15 in concert with $\mathrm{Mg}^{2+}$ resulting in a higher tRNA stability through promoted base paring (41). The integral position of the G15 base in the tRNA structure enables its modification to impact the structural integrity of the molecule, nicely illustrating that the purpose of a modification is tightly associated with its location. This does also apply to base modifications located in the anticodon stem loop of tRNAs. Within the anticodon, the bases at position 34 are 37 are extensively modified (42). The importance of modification in this case becomes clear in their absence, which results in a shift in the translation reading frame, causing transcription of alternative protein sequences (43). This effect may be due to loss of anticodon stability which is normally promoted by anticodon loop modifications (44) such as queuosine, which is located at position 34 of certain tRNAs (33), or the hypermodified base wybutosine (yW) (42). The importance of $\mathrm{yW}$ modification, which has been observed exclusively on position 37 of 
tRNA $^{\text {Phe }}$ for protein translation, was further investigated by Konevega et al., who found yW to promote codon-anticodon interaction through stabilization of tRNA ${ }^{\text {Phe }}$ binding to the ribosomal A site during protein translation (34), thus explaining how absence of this modification can result in frame shifting during translation. Queuine modification and its biological consequences are an important part of this thesis and are described in detail in paragraph 1.5 and following.

Modifications of tRNA molecules in total seem to promote correct function of tRNAs as an integral part of the tRNA molecule. But although the consequences of tRNA modification seem to be generally of beneficial nature, their individual abundance is subject to change. With the level of modification differing from $10 \%$ to over $80 \%$ depending on the type of modification and their location (45), tRNA modifications seem to underly accurate fine tuning mechanisms that may be important for cellular responses to altered environmental conditions. Indeed, the abundance of RNA modifications are subject to change upon the induction of cellular stress as a consequence of reduction of the corresponding RNA modifying enzyme, suggesting that tRNA modifications are also part of the cellular stress response (46). Next to the variety of tRNA modifications, the dynamic change of their abundance adds another layer to this complex biochemical matter. Considering that about 60 million tRNA molecules exist in a mammalian cell (10), the study of tRNA modification and its regulation is highly complex.

\section{$\underline{1.3 \mathrm{M}^{5} \mathrm{C} \text { DNA METHYLTRANSFERASES }}$}

The various modifications on nucleic acids are deposited by "writer" proteins. Specific positions of genomic DNA information have been observed to serve as an acceptor site for methyl groups, transferred from a donor substrate by enzymes, which are therefore called methyltransferases. DNA modification with methyl groups have been observed as N4methylcytosine $\left(\mathrm{m}^{4} \mathrm{C}\right)$, N6-methyladenine $\left(\mathrm{m}^{6} \mathrm{~A}\right)$ and $\mathrm{C} 5$-methylcytosine $\left(\mathrm{m}^{5} \mathrm{C}\right)(47)$. These modifications are not universally distributed among the kingdoms of live as $\mathrm{m}^{4} \mathrm{C}$ modification has only been described in bacteria (48) and $\mathrm{m}^{6} \mathrm{~A}$ levels are most abundant in prokaryotic genomes $(49,50)$. In contrast, $\mathrm{m}^{5} \mathrm{C}$ is the most abundant DNA methylation variant in eukaryotes (51), with the methylation sites often found to belong to a $\mathrm{CpG}$ dinucleotide (52). 
This abundant $\mathrm{m}^{5} \mathrm{C}$ modification is deposited by DNA methyltransferases (Dnmts), which share a conserved catalytic mechanism (Figure 2) to transfer a methyl group from the methyl donor S-adenosyl-methionine (SAM) onto the C5 atom of the nucleic base cytosine.

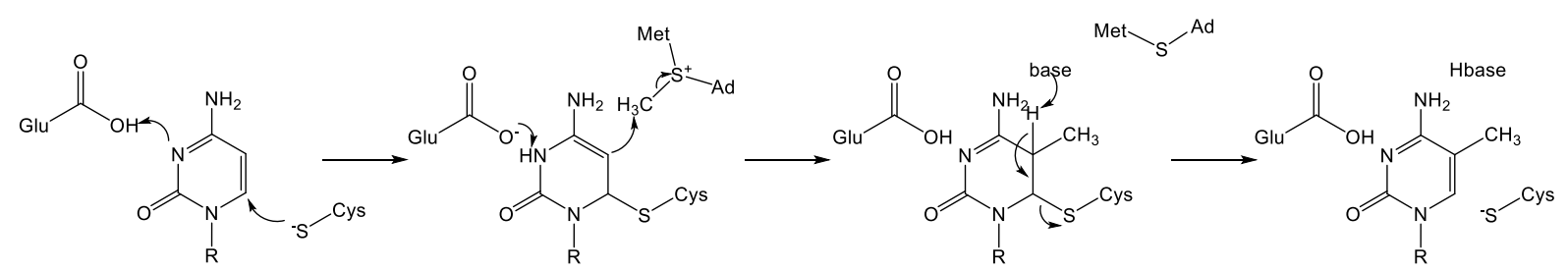

Figure 2. Catalytic mechanism of Dnmt enzymes

In a first step, a covalent enzyme-nucleic acid intermediate is formed through a nucleophilic attack on the cytosine C6 atom by a cysteine side chain that is part of the enzyme's PCQ motive. This attack is aided by a conserved glutamate residue (ENV motive). In a second step, the cytosine`s C5 atom is covalently bound to the SAM`s methyl group, which consequently results in formation of the reaction product SAH (Met-SAd). In a last step, the modified cytosine base is released from the cysteine in a base dependent manner. This figure is based on the previously reported Dnmt mechanism by Jeltsch et al. (53).

Dnmt enzymes are encoded by a broad variety of genomes not only in mammalian organisms, but are also a feature of plants and insects such as the honey bee (52). However, even among the animal kingdom, the set of Dnmt proteins that are encoded in the genome is highly diverse. While five different Dnmts are known in the human system, the extensively studied model organism Drosophila melanogaster is deficient of Dnmt1 and Dnmt3 (54), with no known homologs of this methyltransferases identified. The presence of Dnmt2 as the only Dnmt enzyme make D. melanogaster a valuable tool to study the effects of Dnmt2 in this "Dnmt2 only" organism. Furthermore, there are organisms that show a complete lack of Dnmt enzymes, such as the nematode Cenerohabditis elegans, whose genome does not code for a single Dnmt enzyme (47). 


\subsection{DNMT2}

An extensively studied member of the Dnmt enzyme family is Dnmt2, a protein of about 40 $\mathrm{kDa}$, whose role has emerged to be rather distinct from other members of its enzyme family. The Dnmt2 enzyme shares the same catalytic domain architecture and the catalytic mechanism with other members of the Dnmt family, as of which Dnmt2 has also been suggested to methylate DNA targets like other Dnmts. However, in contrast to the well-studied homologs Dnmt1 and Dnmt3, DNA methylation by Dnmt2 is still controversially discussed. Modification of DNA by Dnmt2 has been investigated employing more than 10 different techniques, including the use of methylation specific antibodies, high pressure liquid chromatography (HPLC), bisulfate sequencing, and even more elaborate approaches such as the combination of HPLC with mass spectrometry $(51,54-58)$. Several of these studies report genome wide methylation of the D. melanogaster genome at a generally low level. However, critical evaluation by Krauss \& Reuter (59), highlights missing controls and technical limitations in some of the aforementioned studies. This view is supported by a more recent study failing to detect specific DNA methylation patterns in D. melanogaster and Schistosoma mansoni in which the authors point out that the low amounts of $\mathrm{m}^{5} \mathrm{C}$ detection level in their experiments is likely caused by technical limitations of the applied bisulfate sequencing technique (54). In general, DNA methylation by Dnmt2 in Drosophila seems to occur at more restricted locations such as retrotransposons or at promotors of specific genes, where it might be involved in the regulation and gene expression respectively (60-63). Despite the controversial discussion about $\mathrm{m}^{5} \mathrm{C}$ methylation in Drosophila, $\mathrm{m}^{5} \mathrm{C}$ methylation seems to be generally absent in the genome of other Dnmt2 only organisms such as $S$. pombe (56).

In contrast to the enigmatic activity of Dnmt2 on DNA, a much higher activity of Dnmt2 was observed for RNA. First evidence for a Dnmt2 target other than DNA came from Goll et al. (64), who used fluorescence microscopy to localize Dnmt2 to the cytoplasm, a finding strongly contradicting the hypothesis of DNA methylation by Dnmt2. As the most striking result, the authors were able to link presence of Dnmt2 in mice to specific methylation of the cytosine base C38 in the anti-codon stem loop of tRNA ${ }^{\text {Asp }}$. A more elaborate approach employing RNA immunoprecipitation from human cell line lysates, confirmed Dnmt2 substrate specificity in the human system and furthermore identified tRNA ${ }^{\mathrm{Gly}}$ and tRNA ${ }^{\mathrm{Val}}$ as additional Dnmt2 targets. Altogether, these three tRNAs are the currently known cognate substrates for Dnmt2 in mammals and are also confirmed in S. pombe (65), however, this substrate specificity does not 
apply to every organism. A different Dnmt2 specificity has been reported for Geobacter sulfurreducens, where investigation of Dnmt2 activity led to the surprising discovery that tRNA $^{\text {Asp }}$ is not methylated by Dnmt2. Instead, tRNA ${ }^{\text {Glu }}$ is a Dnmt 2 target in this organism(66). Furthermore, a weak methylation of tRNA ${ }^{\text {Glu }}$ by Dnmt2 has been also been observed in $S$. pombe (67). While these altered specificities do apply to some organisms, the tRNAs tRNA ${ }^{\text {Asp }}$, tRNA $^{\mathrm{Val}}$ and tRNA ${ }^{\mathrm{Gly}}$ have emerged as the common targets in most organisms, with tRNA ${ }^{\mathrm{Asp}}$ being the most abundant target (68).

A common feature of all Dnmt2 target tRNAs is the presence of a cytosine at the target position 38. However, C38 is not exclusive to Dnmt2 cognate tRNAs, which raises the question how Dnmt2 discriminates cognate from non-cognate tRNAs. Investigation of the binding-surface of the human Dnmt2 enzyme by mutational analysis of basic surface residues revealed that Dnmt2 tRNA interaction might mostly (but not exclusively) be mediated by the anticodon stem and loop (69). However, the sole anticodon stem loop only weakly interacts with Dnmt2 and is not methylated by Dnmt2 $(70,71)$. Consequently, other regions of the tRNA substrate have to contribute to substrate recognition by Dnmt2, which is supported by the observation that also residues more distant from the active site are of importance for Dnmt2 activity (69). A potential candidate region could be the variable loop of the tRNA, as modification by the human Dnmt2 enzyme was lost when the loop was exchanged in the murine tRNA ${ }^{\text {Asp }}$ substrate by the variable loop of the G. sulfurreducens tRNA Asp (66). Also, the tRNA D-loop and L-loop might be involved in substrate recognition. When these regions are replaced with a DNA stretch of the same sequence (containing 3-desoxy-nucleotides) in a tRNA/DNA molecule, the chimeric tRNA is no longer targeted by Dnmt2 (71) indicating that interaction of Dnmt2 might be mediated by the sequence unspecific phosphate ribose backbone in these regions. Interestingly, replacement of C38 and adjacent bases with DNA nucleotides does not negatively impact Dnmt2 activity (71). The observation that Dnmt2 is active on DNA in structural context of tRNA and the importance of other regions of the RNA molecule indicates that specificity may not only be determined by the sequence but that the overall shape of the target is of importance for methylation by Dnmt2. However, the molecular properties of this interaction are still largely unknown. 


\subsubsection{STRUCTURE OF DNMT2 ENZYMES}

To date, three structures of the Dnmt2 enzyme from human (PDB-ID:1G55), Entamoeba histolytica (PDB-ID: 3QV2) and Spodoptera frugiperda (PDB-ID: 4H0N) have been reported $(70,72,73)$. The structure of the human Dnmt2 is presented in figure 3.
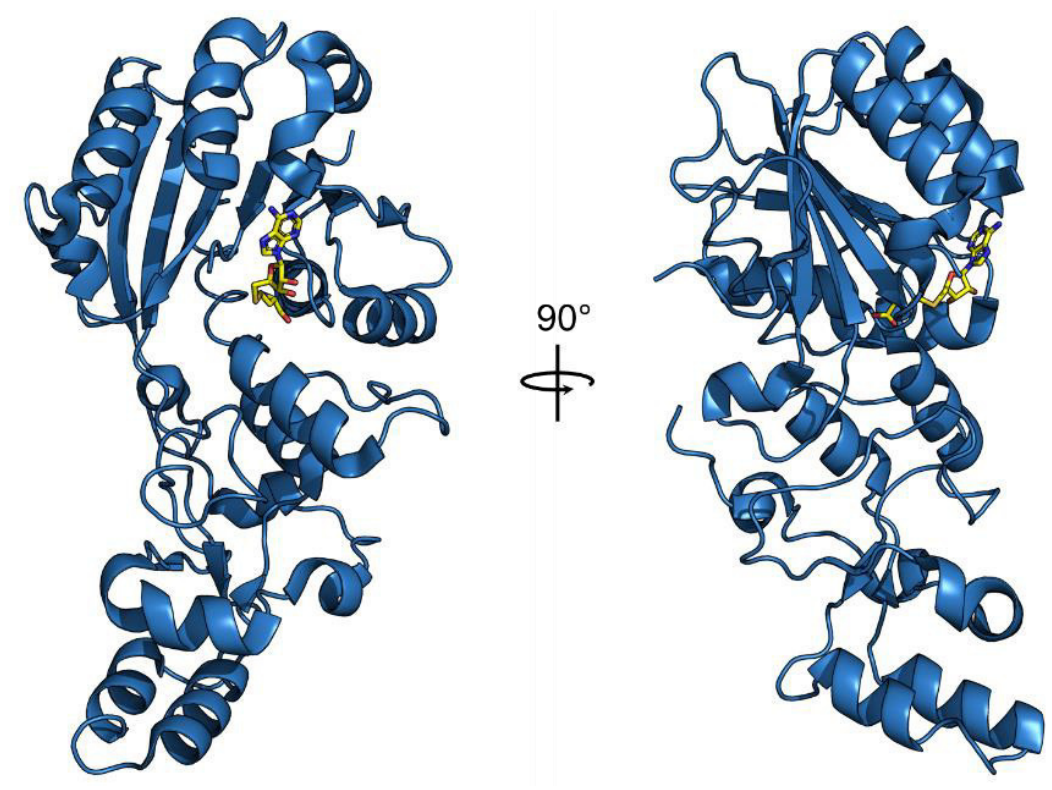

Figure 3. Crystal structure of the Homo sapiens Dnmt2 methyltransferase

The crystal structure of the human Dnmt2 enzyme (PDB-ID: 1G55) is depicted as blue cartoon. The binding pocket of the reaction product SAH (shown as yellow sticks) is formed by the underlying seven stranded $\beta$-sheet. The active site loop, protruding out of the catalytic pocket is not featured by this structure.

All these crystal structures were solved in complex with the reaction product S-adenosylhomocysteine (SAH) that is formed by demethylation of SAM during the enzymatic methyl transfer. However, none of these structures comprises any RNA. Based on these structures, the overall fold and topology of Dnmt2 is highly conserved with a seven stranded $\beta$-sheet as a central structural element that provides the binding pocket for the co-substrate. Differences between the crystal structures are mainly observed in loop regions lacking secondary structures. One prerequisite of Dnmt2 structures seems to be a highly unstructured stretch of about 20 amino acids, which protrudes out of the active site. In the human Dnmt2 structure this loop, including catalytic residue Cys79, is not a featured (72). The S. frugiperda Dnmt2 also possesses the active site loop, but in contrast to the structure of the human enzyme, in this case it is resolved exhibiting two short anti parallel beta-strands (73). This loop conformation is stabilized by an extensive integral interaction with the corresponding active site loop of a 
symmetry mate. Dnmt2 from the pathogenic amoeba E. histolytica also comprises the entire active loop, which here adopts a $\alpha$-helical conformation. This fold, in analogy to the $S$. frugiperda structure, is also stabilized by crystal packing of the $\alpha$-helix (70), however to a much lesser extent. Interestingly, both loops have a different conformation with different secondary structures probably resulting from crystallization. The conformation of this loop in the ternary complex might be different from the crystal structure, as the DNA complex structure of the unrelated, but structurally similar DNA methyltransferase HhaI suggests. Here, the corresponding flexible loop is interacting with the DNA substrate (74), highlighting the possibility that the Dnmt2 active site loop may fulfil a similar purpose (68).

\subsubsection{Biological CONSEQUENCES OF DNMT2 ACTIVITY}

Dnmt2 is the most abundant methyltransferase of the Dnmt family in eukaryotes (75). Despite its high conservation, phenotypes of Dnmt2 deficient model organisms are rather mild and divergent (76), consequently raising the question why Dnmt2 is evolutionary preserved at such a high degree.

Investigation of the function of tRNA methylation by Dnmt2 has provided several insights into biological consequences of methylation by Dnmt2. $\mathrm{m}^{5} \mathrm{C} 38$ is known to protect Dnmt 2 substrate tRNAs from nucleolytic cleavage by the endonuclease angiogenin in Drosophila (77). In opposition, m5C38 hypomodification in Drosophila results in an increase of tRNA degradation products (tsRNAs) produced by endonuclease digest upon heat shock induced cellular stress (78). These tsRNAs lower translation initiation (79) and interfere with activity of the Dicer2 nuclease leading to less production of small interfering RNAs (siRNAs) that consequently results into dysregulation of siRNA pathway dependent genes (78). Furthermore, absence of $\mathrm{m}^{5} \mathrm{C} 38$ modification on tRNA ${ }^{\text {Asp }}$ results in an impaired charging with aspartate in vitro and in reduced synthesis of poly-aspartate containing proteins in Dnmt2 knocked-out mouse embryonic fibroblasts $(80)$.

The consequences of an increased level of tRNA degradation products, resulting from reduced levels or absence of $\mathrm{m}^{5} \mathrm{C} 38$, are not the sole downstream effects of this modification. Moreover, this modification itself is impacting protein translation. Dnmt2 specifically methylates tRNA $^{\text {Asp }}$, tRNA $^{\text {Gly }}$ and tRNA ${ }^{\mathrm{Val}}$ in human and Drosophila at C38 position in the anticodon stem loop $(64,81)$. Modifications in this tRNA region, especially of the wobble base, have been shown to impact protein translation through pre-structuring the anticodon and increasing tRNA 
stability to ensure correct recognition of the tRNA by the ribosome (42). The close proximity of the Dnmt2 methylation target site to the anticodon and to the wobble base, raises the possibility that also $\mathrm{m}^{5} \mathrm{C} 38$ modification of these tRNAs may affect protein translation (68). First indications for a function of Dnmt 2 mediated tRNA methylation came from double knock out mice, deficient of Dnmt2 and NSun2, a ${ }^{5} \mathrm{C}$ tRNA methyltransferase with a distinct target specificity (82). In these Dnmt2 $2^{-{ }^{-}}, N S u n 2^{-{ }^{-}}$mice, protein biosynthesis as well as tRNA levels were substantially reduced while mRNA levels remained unchanged compared to the wild type (WT) (83). Furthermore, ribosome foot printing of phenotypically affected tissues derived from Dnmt2 $^{-/-}$only mice revealed that C38 methylation is required for codon fidelity. Absence of $\mathrm{m}^{5} \mathrm{C} 38$ leads to an increased misinterpretation of the near cognate codons CAG and CAC, which code for aspartate and glutamate respectively, resulting in mutual misincorporation of the two amino acids. The result is an increased degradation of these, presumably misfolded, proteins by the ubiquitin proteasome machinery (84). Additionally, Dnmt2 activity is required for correct differentiation of bone marrow multipotent stem cells (MSCs), in which absence of Dnmt 2 results in a hematopoietic defect causing impaired differentiation. These findings accumulate into the hypothesis that Dnmt2 aids differentiation by modulated expression of certain proteins through adapting tRNAs to codons (84).

Recent results suggest that tsRNAs do not only affect protein translation in one generation alone, but are involved in intergenerational transmission of paternally acquired phenotypes in a Dnmt2 dependent but DNA independent manner $(85,86)$. Application of a high-fat diet (HFD) to mice results in an upregulation of Dnmt2 in parts of the male reproductive tract, where sperm cells maturate. In consequence, upregulation of Dnmt 2 does not only tune tsRNA levels, but also correlates with an increase of $\mathrm{m}^{5} \mathrm{C}$ methylation levels on sperm cell small non-coding RNAs. This RNA fraction contains transcripts of about 30-40 nucleotides and is mainly composed of tsRNAs (85), with degradation products from tRNA ${ }^{\text {Glu }}$ being the most abundant tsRNA in sperm $(87,88)$. Injection of this RNA fraction, isolated from sperm of mice kept under a high-fat-diet, into oocytes, caused development of a metabolic disorder in the offspring generation (87). In contrast, deletion of Dnmt2 prevented elevated $\mathrm{m}^{5} \mathrm{C}$ modification in this RNA fraction and abolished transmission of the HFD induced metabolic disorders (86). Furthermore, this study shows that methylation of the tsRNAs alters their secondary structure properties arguing that Dnmt2 activity contributes to a secondary level of paternal hereditary information (86). Besides HDF induced disorders, other paternal hereditary phenotypes have been shown to be transmitted intergenerationally by RNAs $(89,90)$. These novel findings reveal 
previously unidentified functions of Dnmt2 and harbor the potential of new discoveries that may further contribute to the understanding why Dnmt2 is strictly conserved (86).

\subsection{QUEUINE}

In 1968 a previously unknown nucleoside was identified in the tRNA ${ }^{\text {Tyr }}$ anticodon of the gram negative bacterium Escherichia coli that leads to suppression of a stop codon and instead to incorporation of a tyrosine into the polypeptide chain (91) (92). The nucleoside was assigned the letter Q, with the base called queuine and the nucleoside named queuosine (93). Later, this nucleoside was also identified on three other tRNAs (tRNA ${ }^{\text {Asp }}$, tRNA ${ }^{\text {Asn }}$ and tRNA ${ }^{\text {His }}$ ) located at the wobble base same position (33). In following investigations, the structure of queuosine (Figure 4) was determined by gas chromatography, mass spectrometry (MS) and nuclear magnetic resonance (NMR) $(94,95)$.

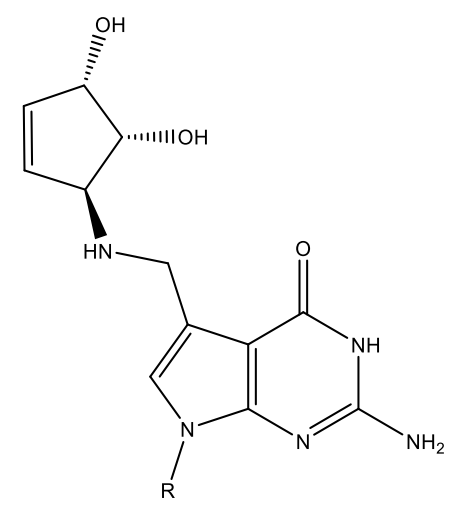

Figure 4. Chemical structure of queuosine

The ribose is substituted by the letter $\mathrm{R}$ in the structure

Queuosine was identified to be a modified guanosine analogue in which the N7 is substituted by a carbon atom. At this carbon an aminomethlyether bridge links to a ring of five carbon atoms. In this ring a double bond as well as two hydroxy groups are present at C4 and C5 position which exhibit a cis-conformation completing the structure of this cyclopentene-diol. Currently this 7-(4,5-dihydroxyl-1-1-cyclopentene-3-yl-aminomethyl)-7-deazaguanine is referred to as queuine (96). 


\subsubsection{QUEUINE BIOSYNTHESIS}

Early investigation of the queuine content in E.coli found no free queuine base in extracts, arguing that queuine is not synthesized as a free base in the bacterium's cytoplasm (97). To date, no direct queuine synthesis has been observed in any organism (98). Instead, the queuine precursor, 7-ammoniomethyl-7-deazaguanine ( $\mathrm{preQ}_{1}$ ), is synthesized in the bacterial cytoplasm by five enzymatic reactions before it is incorporated into tRNA by the bacterial tRNA guanine transglycosylase enzyme (Figure 5) (97).

The biosynthesis of preQ ${ }_{1}$ in bacteria is a multi-step process involving five different enzymes of several enzyme classes. The first step of preQ ${ }_{1}$ synthesis is catalyzed by the first enzyme of the folate pathway, GTP cyclohydrolase I (99). Starting from guanosine-5'-triphosphate, this enzyme, which is encoded by the FolE gene in E.coli (100), catalyzes the cleavage of the imidazole ring at the $\mathrm{C} 8$ via protonation of the $\mathrm{N} 7$ atom and cleavage of the furanose ring, generating a Schiff-base from the glycoside in a proposed concerted action (101). Followed by a rearrangement and subsequent re-cyclization, a pterin ring is formed, finalizing the reaction product 7,8-dihydroneopterin triphosphate $\left(\mathrm{H}_{2} \mathrm{NTP}\right)(101,102)$. This product is a substrate for the QueD enzyme (6-Carboxy-5,6,7,8-tetrahydropterin synthase), a homohexameric protein that binds the pterin moiety at one of its six active sites all located at the monomer interfaces as observed in the QueD crystal structure $(103,104)$. QueD catalyzes the turnover of $\mathrm{H}_{2} \mathrm{NTP}$ into the reaction product 6-carboxy-5,6,7,8-tetrahydropterin $\left(\mathrm{CPH}_{4}\right)$ under generation of the leaving group acetaldehyde (103). This reaction is succeeded by the QueE enzyme and metal ion dependent ring contraction of $\mathrm{CPH} 4$ into a guanine derivative, in which the $\mathrm{N} 7$ is replaced by a carbon atom with a covalently linked carboxy group (7-carboxy-7-deazaguanine) in a SAM dependent manner (105). The carboxy group of 7-carboxy-7-deazaguanine has to be converted into a nitrile to yield preQ $\mathrm{Q}_{0}$. The underlying reaction is catalyzed by the hydrolase QueC in an ATP dependent step in which the cyano nitrogen is derived from ammonia $(106,107)$. The crystal structure of QueC from Bacillus subtilis reveals a homotetrameric architecture of the enzyme with each monomer containing a zinc ion tetragonally coordinated by three cysteines (PDB-ID: 3BL5) (108). Despite the quaternary assembly in the crystal, just the monomer is annotated as the biological assembly, as it contains the substrate binding cleft. However, whether the QueC quaternary structure contributes to catalysis and the role of the zinc binding domain has yet to be determined. The unprecedented reduction of the preQ $_{0}$ nitrile to the primary amine of preQ $_{1}$ is promoted by another enzyme of the queuine34 tRNA synthetic pathway, the QueF transferase (109). This enzyme exhibits a homodecameric, quaternary 
structure with two head to head facing pentameric assemblies providing ten catalytic centers at their interfaces (PDB-ID: 4F8B) (110). The energy necessary for the cleavage of two of the N$\mathrm{C}$ bonds is provided by the oxidation of two NADPH/ $/ \mathrm{H}^{+}$molecules (109). The queuine precursor preQ $_{1}$ is then incorporated into the tRNA substrate at the wobble base position by the bacterial TGT enzyme, which is described in more detail in paragraph 1.6.1.

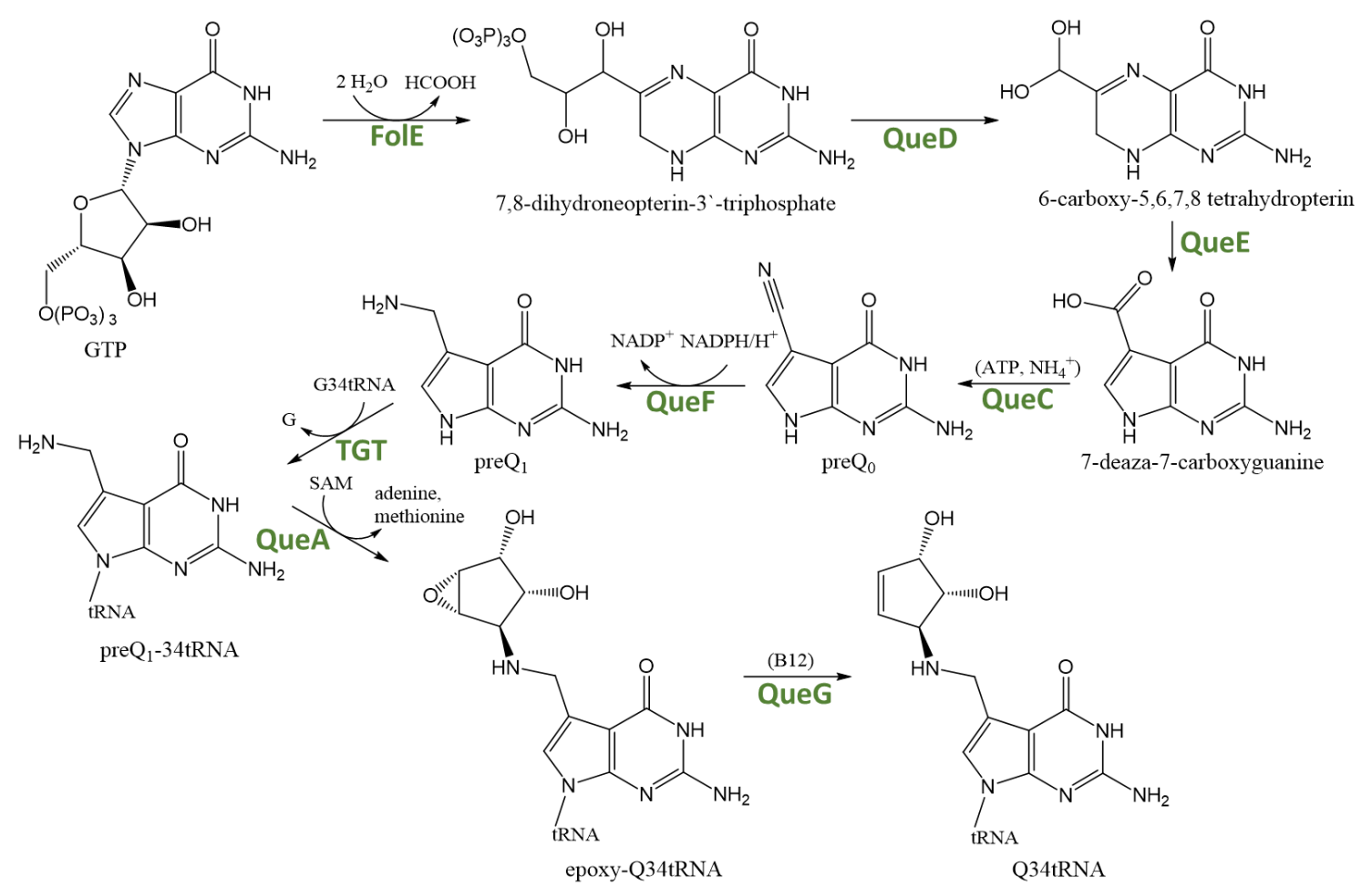

Figure 5. Biosynthesis of pre $\mathrm{Q}_{1}$ and $\mathrm{Q}_{34 \mathrm{tRNA}}$ in bacteria

The queuine precursor pre $\mathrm{Q}_{1}$, which is incorporated at position 34 into cognate tRNAs by the tRNA guanine transglycosylase enzyme is built up from guanosine 5'-triphosphate (GTP) in bacteria in a sequential reaction catalyzed by the five enzymes FolE, QueE, QueC and QueF. After generation of preQ $1-34$ tRNA, the 7-deaza-guanine derivative is further modified under use of SAM by QueA. The final queuine modification is established by QueG.

After generation of the preQ ${ }_{1}-\mathrm{G} 34 \mathrm{tRNA}$, the fully modified queuine base needs to be established on the tRNA. The first step of the addition of the cyclopentene-cis-diol ring to the modified base is facilitated in a complex reaction by the tRNA ribosyltransferase isomerase (QueA) consisting of two domains one of which comprises a 6-stranded $\beta$-barrel $(111,112)$. The preQ ${ }_{1}$ tRNA and the SAM is sequentially bound, forming a ternary complex followed by the reaction and the release to the epoxy-queuosine tRNA (epoxy-Q34tRNA) (113). In a last 
step, a two-electron reduction results in removal of the epoxide and subsequent formation of the characteristic cyclopentene-diol moiety, finalizing the Q34tRNA synthetic pathway with the fully established queuine tRNA modification. The enzyme catalyzing this final reaction is the epoxy-queuosine reductase QueG (114). The structure of the QueG enzyme (PDB-ID: 5D08) reveals a cob(II)alamine and two 4Fe4S clusters at the active site (115). Both of them were identified to be catalytically essential in an earlier study (114). Lately, a structural study by Dowling et al. revealed a convincing model for opening of the epoxide until the release of the final queuine modified tRNA molecule. Reduction of the cobalamine's $\mathrm{Co}^{2+}$ to $\mathrm{Co}^{+}$likely involving action of the $\mathrm{Fe}_{4} \mathrm{~S}_{4}$ cluster, marks the start of this reaction. Subsequently, $\mathrm{Co}^{+}$is able to perform a nucleophilic attack on the epoxide ring forming a covalent Co-alkyl intermediate, which results in the opening of the epoxide. Upon reduction of $\mathrm{Co}^{3+}$ to $\mathrm{Co}^{2+}$ and condensation of a water molecule, the characteristic double bond in the queuine's cyclopentene moiety is established and the final reaction product of the queuosine biosynthetic pathway, Q34tRNA, is released (116).

\subsubsection{QueUine UPTAKE IN PROKARYOTES}

In total, the synthesis of queuine and its precursors involves multiple enzymes, different cosubstrates and even a vitamin. This complex cascade poses a substantial energetic burden for the cell and lowering of the energetic costs without sacrificing the benefits of queuine modification might be beneficial to the cells energy management. Therefore, the uptake of queuine from the environment surrounding the cell is an obvious way to circumvent queuine biosynthesis. Furthermore, not all bacteria are capable to synthesize queuine by themselves as they lack enzymes of the queuine biosynthetic pathway (117). These organisms rely on scavenging queuine precursors which in consequence requires a mechanism that facilitates their uptake. Interestingly, E. coli has been observed not only to synthesize queuine and its precursors itself, but also to salvage $\operatorname{pre}_{0}$ and preQ $_{1}$ from an extracellular source. Not only the uptake of the precursors has been observed, but it was shown by comparative genomics analyses to involve the COG1738 member YhhQ. In the experimental setup this transporter was essential for transport of the queuine precursor $\mathrm{preQ}_{0}$ into the cell. However, whether this transporter is directly facilitating the uptake of $\mathrm{preQ}_{0}$ or facilitates another essential step of the uptake mechanism remains unknown (118). Furthermore, YhhQ seems not to affect preQ ${ }_{1}$ uptake, pointing out how little of the uptake mechanism is understood so far. 


\subsubsection{QUEUINE UPTAKE IN EUKARYOTES}

No homologous proteins for bacterial enzymes involved in the queuine biosynthetic pathway have been identified in eukaryotes. Therefore, deficiency of queuine biosynthesis in eukaryotic organisms has been the accepted opinion in the field for quite some time, although this may not be a definitive proof of absence. However several studies show that eukaryotic organisms, such as Drosophila melanogaster, Caenorhabditis elegans, Dictyostelium discoideum and the eukaryotic algae Chlorella pyrenoidose as well as Chlamydomonas reinhardtii can be fully depleted of their queuine pool, if held strictly under queuine deficient conditions for an extended time period (119-122) arguing that in these organisms indeed do not synthesize queuine. Full depletion of Q-tRNA from the organism can take as long as one year after start of the queuine free diet, as has been shown for mice held under axenic (germ free) conditions (123), indicating the presence of a highly efficient recycling system for this essential base in eukaryotes.

The apparent disability of eukaryotes to synthesize the queuine base consequently makes them rely on the content of this essential nutrient in extracellular sources. However, the amount of queuine required by the human organism might be minimal as is suggested by the low content of queuine in human milk ( $1 \mathrm{ng} / \mathrm{mL}$ ) (98) arguing for a highly efficient system present in men to keep this modified base inside the organism. Investigation of the queuine content of dietary products, such as plant derived sources or dairy products, finds them to contain sufficient amounts of queuine to meet the human dietary needs (98). The amount of queuine in nutritional sources is highly variable ranging from $1 \mathrm{ng} / \mathrm{mL}$ in evaporated and canned goat milk over 21 $\mathrm{ng} / \mathrm{g}$ in tomato up to $87-530 \mathrm{ng} / \mathrm{mL}$ in coconut water. The high content of queuine especially in plants raises the question how it traverses from the bacterial to the eukaryotic organism. To animals, queuine sources may be exposed in the gut environment upon the normal rate of the microbial turnover in the intestine (124), while plants seem to rely on the bacterial turnover in soil. The transfer of queuine sources might be promoted by the specificity of the eubacterial TGT enzyme, which does not accept queuine, queuosine nor the queuine nucleotide as substrates $(125,126)$.

Despite the disability of eukaryotic cells to synthesize queuine, the observation that queuine is found incorporated in the tRNA of eukaryotic organisms implies the uptake of peripheral queuine into the eukaryotic cell as a logical consequence. Indeed, the concentration of queuine circulating in the human system has been estimated to be in the range of 1-10 $\mathrm{nM}(127,128)$. In analogy to bacteria, queuine uptake in eukaryotes is still largely unclear. Studies trying to 
decipher the mechanism of queuine uptake are exploiting the properties of a reduced queuine tritium derivative $\left(\mathrm{rQT}_{3}\right)$, which is not a substrate for the human TGT but gets efficiently taken up into the cell at a rate comparable to queuine, making this compound suitable for investigation of queuine uptake (129-131). A study by Elliot et al. focused on quantitative evaluation of the uptake of queuine into cultured human foreskin fibroblasts (HFF) cells, describes two phases of queuine salvation. They monitored the cellular $\mathrm{rQT}_{3}$ concentration over time after its application to the culture, which resulted in a diphasic diagram. Initially after $\mathrm{rQT}_{3}$ application a first uptake system is responsible for a quick saturation of the $\mathrm{rQT}_{3}$ cellular concentration after 2-4 min with a $\mathrm{K}_{\mathrm{m}}$ value of about $30 \mathrm{mM}$, while another uptake system with an estimated $\mathrm{K}_{\mathrm{m}}$ of $350 \mathrm{mM}$ is responsible for an steady increase of the $\mathrm{rQT}_{3}$ concentration, which saturates at $1 \mu \mathrm{M}$ after 3-4 hours (132). Despite the apparent queuine uptake in eukaryotes, the components underlying these two systems are yet to be described.

\subsubsection{FURTHER MODIFICATION OF THE QUEUINE BASE}

GTP is extensively modified to preQ $_{1}$ before it gets incorporated into target $\mathrm{tRNAs}$ in bacteria but does not represent the final modification, because the fully established queuine bases possesses an additional cyclopentene-diol moiety, that is formed after incorporation of the preQ $_{1}$ into the tRNA target. However, the final queuine modification does not necessarily represent the final modification stage of this base. The majority of queuine molecules derived from rat, rabbit and starfish carry an additional modification, themed as $\mathrm{Q}^{*}$, which was observed by means of thin layer chromatography and mass spectrometry. Although frequently found in mammalian samples, queuine but no $Q^{*}$ species are observed in samples derived from plants, such as wheat germ (133). MS experiments with tRNA from rat liver showed a mass shift of queuine by $571 \mathrm{~m} / \mathrm{z}$, caused by O-linked mannosylation (manQ) and/or galactosylation (galQ) of the cyclopentene-diol moiety. While mannose modification is exclusively overserved for $\mathrm{tRNA}^{\mathrm{Asp}}$, queuine in $\mathrm{tRNA}^{\mathrm{Tyr}}$ carries an additional galactose. $\mathrm{tRNA}^{\mathrm{Asn}}$ and $\mathrm{tRNA}^{\mathrm{His}}$, although being queuinylated, are not further targeted by glycosyltransferases. Despite Omannosylation and O-galactosylation of queuine occurs on different tRNAs, they both are localized at the $\mathrm{C} 4 \mathrm{OH}$ group of the cyclopentene, as observed by proton resonance shift of the hypermodified base in comparison to the unmodified queuine in NMR (134). A further study revealed that queuine hypermodification in eukaryotes is not only restricted to rodents or asteroidea, but is also present in human (64). Here, tRNA ${ }^{\text {Asp }}$ harbors manQ at the wobble base position. 
The glycosyltransferase(s) modifying queuine in the structural context of tRNA are yet unknown. However, a mannosyltransferase was isolated from cell free rat liver extract by chromatography and enzymatically characterized by using the substrate uridine diphosphate (UDP) - $\alpha$-mannose as a donor. Mannosylation activity of this enzyme was specific for the queuine base in structural context of tRNA ${ }^{\text {Asp }}$ and no other Q34tRNAs (tRNA ${ }^{\text {Asn }}$ tRNA $^{\text {His }}$, tRNA $^{\text {Tyr }}$ ) were modified (135). Furthermore, the authors found the transfer of mannose on queuine to be crucially dependent on the presence of $\mathrm{Mg}^{2+}$, an observation that is in line with the current knowledge that most enzymes of this class rely on $\mathrm{Mg}^{2+}$ or $\mathrm{Mn}^{2+}$ as a cofactor. These divalent metal ions are supposed to promote leaving of the nucleoside diphosphate group by stabilizing the developing negative charge (136).

\subsection{TRNA GUANINE TRANSGLYCOSYLASES}

Transfer RNA guanine transglycosylases (TGTs) are tRNA modifying enzymes that are encoded in archaebacteria, eubacteria and eukaryotes where they catalyze the incorporation of 7-substituted 7-deazaguanine derivatives into cognate tRNAs (137). While the 7-deazaguanine core moiety of the substrate is the same for every TGT, the base accepted by the according enzyme differs in the substituent at position 7 of the 7-deazaguanine which is specific to the kingdom of the respective organism (138). Despite their differences in substrate specificity, TGT enzymes share a fairly similar fold (139-141), that is mainly constituted of an $(\beta / \alpha)_{8}$ TIM barrel which provides elements involved in substrate binding and its recognition $(142,143)$. Substrate recognition is further facilitated by the second prominent structural element of TGT enzymes, a zinc binding domain $\mathrm{N}$-terminally to the barrel, comprising a divalent zinc ion $\left(\mathrm{Zn}^{2+}\right)$. This overall highly similar fold classifies TGTs as an own superfamily (EC 2.4.2.29).

Members of this superfamily can be divided in eubacterial, archaebacterial und eukaryotic TGTs that differ in the modified base they transfer, tRNA substrate specificity and/or the target site (Figure 6). 


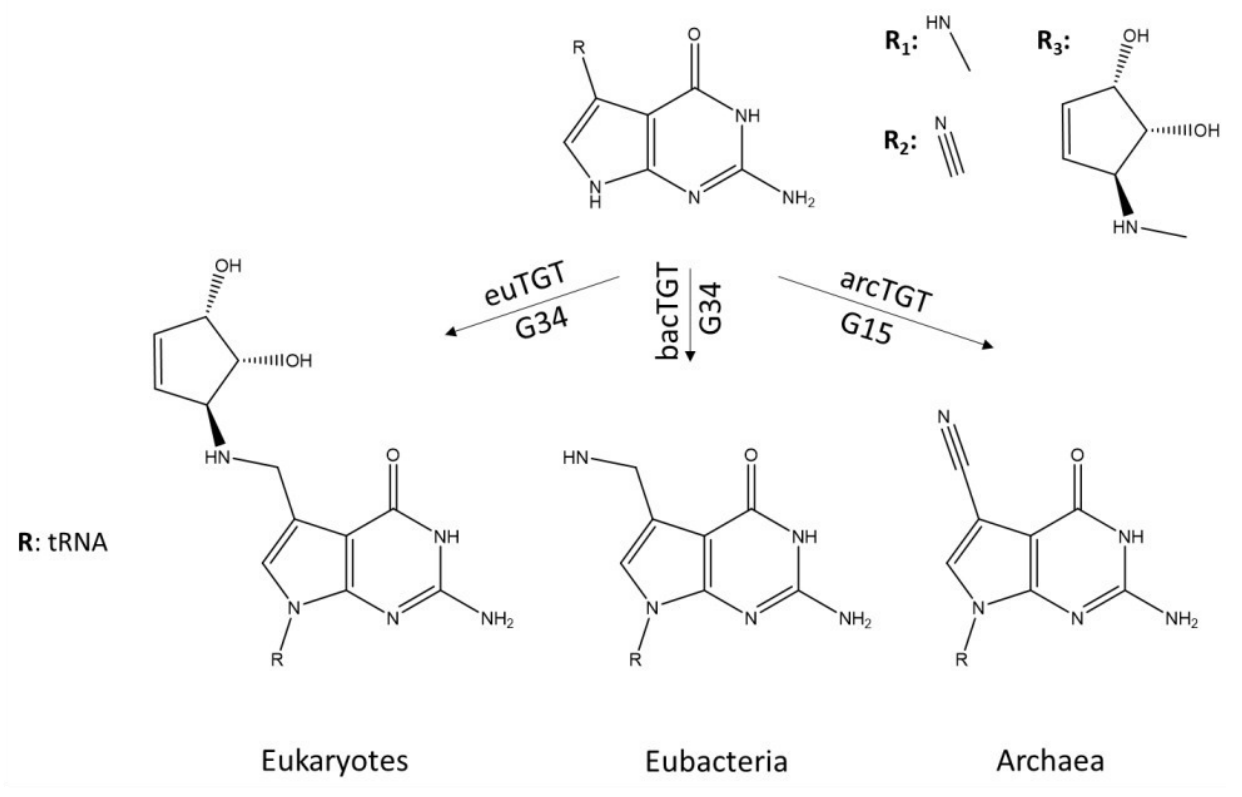

Figure 6. Kingdom specific incorporation of 7-deazaguanine derivatives by TGTs

With respect to the kingdom, the bacterial TGT (bacTGT), the eukaryotic TGT (euTGT) and the archaeal TGT (arcTGT) incorporate preQ ${ }_{1}\left(\mathrm{R}_{1}\right)$, preQ $\mathrm{Q}_{0}\left(\mathrm{R}_{2}\right)$ or queuine $\left(\mathrm{R}_{3}\right)$ into cognate tRNAs respectively. While the bacTGT as well as the euTGT target the guanine at the wobble base position (G34), the arcTGT exhibits a different specificity and exchanges G15 with the modified base.

\subsubsection{THE EUBACTERIAL TGT}

TGTs of eubacterial organisms (bacTGTs) incorporate the queuine precursor 7-aminomethyl7-deazaguanine or preQ ${ }_{1}$ into the cognate tRNAs tRNA ${ }^{\text {Asp }}$, $\mathrm{tRNA}^{\text {Asn }}$, $\mathrm{tRNA}^{\mathrm{His}}$ and tRNA ${ }^{\mathrm{Tyr}}$ (125). These tRNAs share their anticodon sequence, G34 U35 N36, with N representing one of the four canonical nucleotides ( $\mathrm{G}, \mathrm{C}, \mathrm{A}$ or $\mathrm{U}$ ), with the triplet nucleotide sequence U33 G34 and U35 representing the minimal recognition sequence for bacTGT $(144,145)$. Next to the sequence, the tertiary structure to the acceptor tRNA is of importance for the substrate recognition by TGTs, as has been observed for a truncated helix mimicking the anticodon of a tRNA substrate. This helix serves as a substrate for TGT at $20^{\circ} \mathrm{C}$, but at $37^{\circ} \mathrm{C}$ no TGT activity is observed on this substrate anymore, due to loss of the substrate's secondary and tertiary structures (145).

The two structures of a bacTGT currently available are the crystal structure of Thermatoga maritima bacTGT (PDB-ID: 1ASH) and Zymomonas mobilis (PDB-ID: 1PUD) (139) latter of which is the structurally most extensively studied enzyme of the TGT superfamily with currently over 100 entries in the PDB database. 


\subsubsection{STRUCTURE OF THE BACTERIAL TGT}

First insights into the structure of a TGT enzyme were provided by the crystal structure of the Z. mobilis TGT by Romier et al. (139), who observed the bacTGT's characteristic $(\beta / \alpha)_{8}$ to be capped at the $\mathrm{N}$-terminal site with a three-stranded $\beta$-sheet, belonging to the $\mathrm{N}$-terminus of the protein (Figure 7).

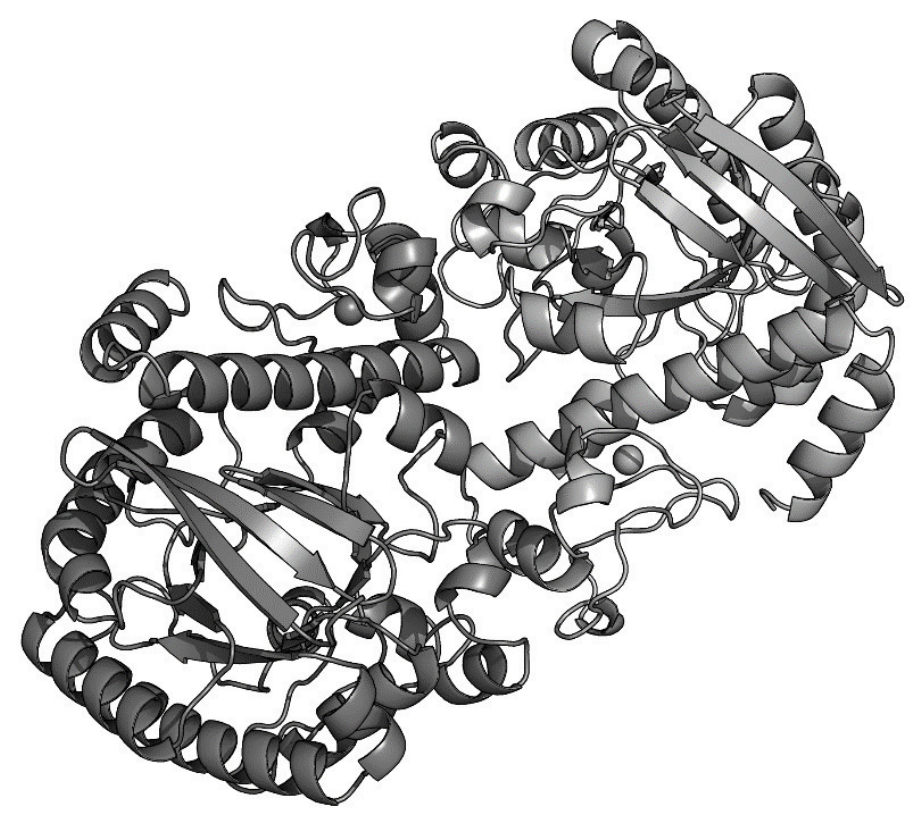

Figure 7. Crystal structure of the Z. mobilis bacTGT assembly

The functional homodimeric assembly of the bacTGT from Z. mobilis (PDB-ID: 1PUD) is shown with both subunits depicted as cartoon. The two identical subunits are colored individually in light- and dark grey.

The zinc atom of each subunit it shown as a sphere and colored accordingly to the subunit.

Furthermore, they observed that the $\beta$-strands of the barrel are not only linked by one $\alpha$-helix connecting two sheets, but in some cases by multiple helices or even an additional threestranded beta sheet. The barrel is bridged by an $\alpha$-helix to a C-terminal domain, which features a structurally important zinc ion that is coordinated by three cysteines and an $\alpha$-helix bound histidine, stacking from the top to complete the tetragonal coordination of the metal ion. This binding motive can be described as $\mathrm{CXCX}_{2} \mathrm{CX}_{29} \mathrm{H}$, and is conserved among bacTGTs (139). 


\subsubsection{STRUCTURE OF THE BACTERIAL TGT SUBSTRATE COMPLEX}

Crystallization of protein RNA complexes is a challenging task, as protein RNA interactions are usually transient and exhibit low binding constants, which hinders crystallization. To overcome this problem, researchers have been using modified bases or cross linking to stall the enzymatic reaction at a distinct point in the reaction cycle and thus trap the protein RNA complex as covalent reaction intermediate $(146,147)$.

To trap the Z. mobilis TGT RNA reaction intermediate, Xie et al. (142) used 9-deaza-guanine $(9 \mathrm{dzG})$, which prevents bond formation between the modified base and the ribose. By using this modified base, they were able to stall the enzymatic reaction after cleavage of the glycosidic bond and crystallized the complex with a covalent bond formed between the ribose and the Asp 280 residue of the TGT (Figure 8). This crystal structure offers first insights into RNA binding by a bacTGT. In the complex structure, TGT forms a homodimer, with $9 \mathrm{dzG}$ bound to the active site of both subunits. However, only one subunit has the RNA, which is comprised of an tRNA anticodon stem anticodon loop, bound to its active site. The question why only one subunit contains the tRNA substrate, cannot be resolved by this structure, but in presence of the full length tRNA, the second subunit might aid binding of the RNA substrate, as it is the case for the arcTGT (143). In contrast to the arcTGT (Chapter 1.6.2), both active sites in the homodimer are facing a similar direction, a fact that might obstruct binding of a second tRNA substrate to the unoccupied second catalytic center, due to steric hinderance by the tRNA bound to the other subunit (142). The fold adopted by the bacTGT monomer in the ternary complex is fairly similar to the bacTGT crystal structure without RNA. In the complex structure the RNA sits on top of the $\mathrm{C}$-terminal end of the $(\beta / \alpha)_{8}$ barrel, representing the conformation of a tRNA anticodon stem and loop. The stem interacts with the enzyme in a sequence unspecific manner via van der Waals contacts and hydrogen bonds involving not the bases but the phosphate backbone. In contrast to the stem, the anticodon loop adopts an unusual conformation that is subjected to a global conformational change upon binding, compared to the unbound tRNA full length structure. Furthermore, the loop is engaged in extensive interaction involving the bases C32A38 (142). 


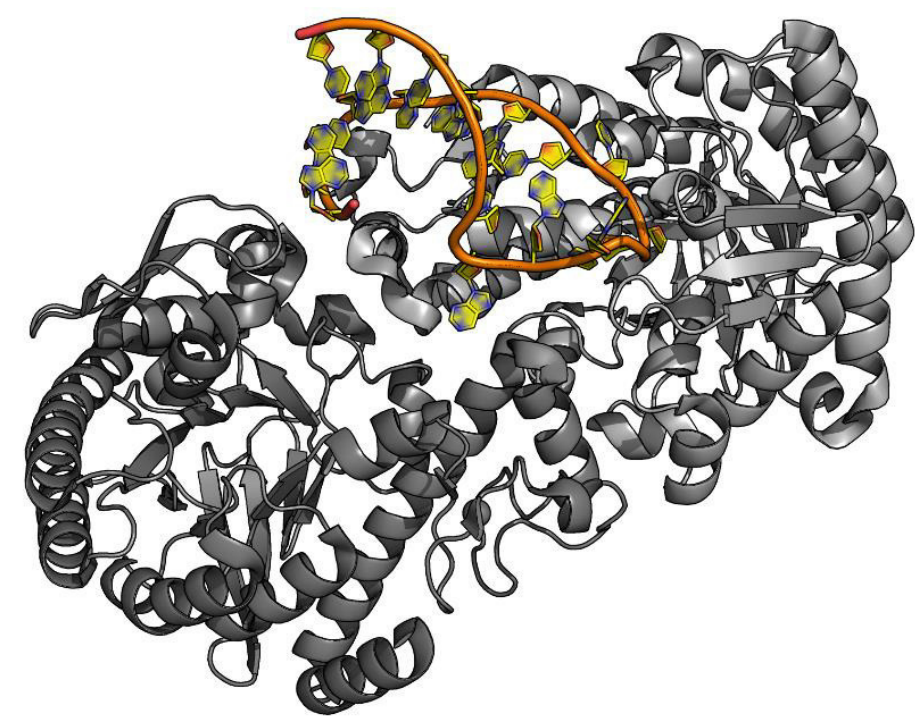

Figure 8. Crystal structure of a bacTGT RNA complex

The bacTGT from Z.mobilis (PDB-ID: 1Q2R) is shown in complex with a tRNA anticodon stem loop all depicted as cartoon. The RNA is bound exclusively to the active site of one subunit (light grey) while the active site of the homodimer's other subunit (dark grey) in unoccupied. All molecules are shown as cartoon, while the zinc ion of each monomer is depicted as a sphere in the respective color of the subunit.

\subsubsection{THE ARCHAEBACTERIAL TGT}

TGT enzymes found in archaea (arcTGTs) exhibit outstanding properties that clearly distinguish them from their eubacterial and eukaryotic counterparts. Sharing only 20-25\% sequence identity with bacTGTs (148), the most remarkable difference is the completely different target site, which is the guanine 15, located in the D-arm of most archaeal tRNAs (15, 149). Compared to the bacTGT, archaeal TGTs do not incorporate preQ $\mathrm{Q}_{1}$, but 7-cyano-7deazagunanine or preQ $\mathrm{Q}_{0}$, that harbors a nitrile group as part of the substituent at position 7 (150). In contrast to eubacteria where $\operatorname{preQ}_{1}$, which is subsequently modified into queuine on tRNA, in archaea preQ $_{0}$ tRNA is converted into 7-formamidino-7-deazaguanine-tRNA in a single enzymatic reaction (151). Because of the occurrence of this modified base on the tRNA of archaebacteria it is referred to as archaeosine or $\mathrm{G}^{*}$. Archaeosine modification is thought to promote tRNA stability, a crucial task for thermophile archaeal bacteria such as Pyrococcus horikoshii $(36,138)$ 


\subsubsection{STRUCTURE OF THE ARCHAEBACTERIAL TGT}

The first structure of an arcTGT was described for the TGT from the thermophile organism $P$. horikoshii (PDB-ID: 1IQ8) (Figure 9) (140). This arcTGT comprises the characteristic $(\beta / \alpha)_{8}$ barrel and the zinc binding domain, which, together with the shared catalytic mechanism, clearly identify this enzyme as a member of the TGT enzyme family. Furthermore, arcTGT is organized in a homodimeric assembly with the zinc binding domain heavily involved in dimer formation. Although the arcTGT structure shares several similarities to queuosine TGTs, it exhibits pronounced structural differences. The arcTGT contains three additional domains at the C-terminus, consequently named C1-C3 that are specific to archaeal TGTs and completely absent in queuosine TGTs. Interestingly, the binding site for the $\mathrm{pre}_{0}$ substrate is provided by an $\alpha$-helix which is unfolded in the free state and adopts a fold upon binding of the modified base (140).

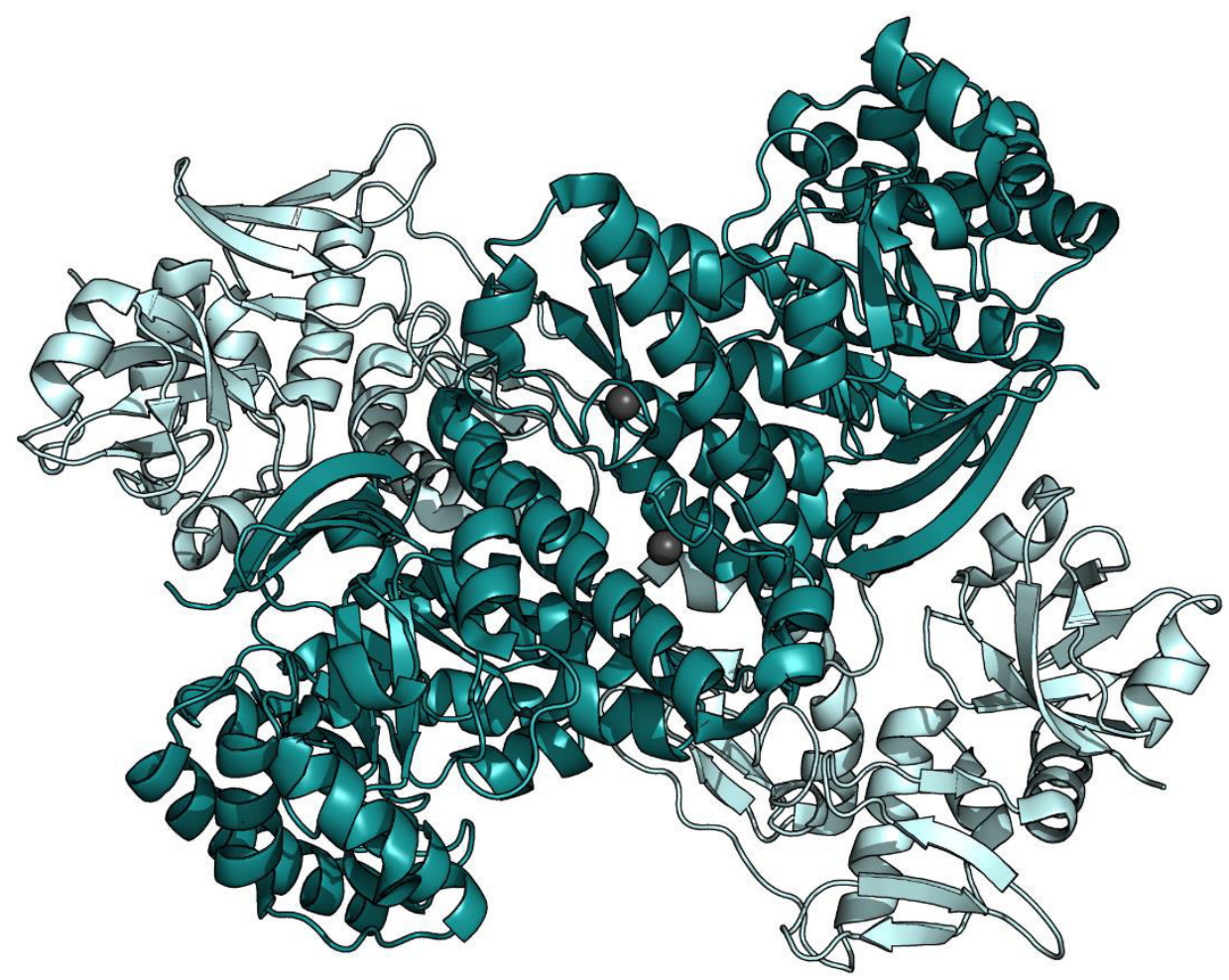

Figure 9. Crystal structure of the $P$. horikoshii arcTGT homodimer

Both subunits of the P. horikoshii arcTGT homodimer crystal structure (PDB-ID: 1IQ8) are depicted as cartoon and colored identically. The N-terminal domain, which resembles the fold of the bacTGT, is colored in turquoise, while the three C-terminal domains $\mathrm{C} 1-\mathrm{C} 3$, that are not featured by TGTs from other kingdoms are shown in pale turquoise. The zinc atoms that are coordinated by each subunit individually are presented as grey spheres. 
The crystal structure of the $P$. horikoshii arcTGT homodimer in complex with a full length tRNA (PDB-ID: 1J2B) describes a novel tRNA conformation. In the free tRNA, the G15 target base is buried in the D-loop of the L-shaped tRNA molecule (PDB-ID: 1EHZ) (152). The arcTGT tRNA complex structure (Figure 10) reveals the same homodimeric assembly as in the tRNA-free structure of the arcTGT, but to access G15 for the enzymatic reaction, the tRNA is forced into a different conformation by the arcTGT, referred to as the $\lambda$-conformation. Promoted by the C-terminal domains, this tRNA shape exposes bases of the D-loop to the protein surface and allows to insert the G15 base into the active site of the arcTGT. In contrast to the bacTGT, two tRNA molecules are bound to the homodimer without steric hinderance in the complex structure with one G15 accommodated in each catalytic pocket.

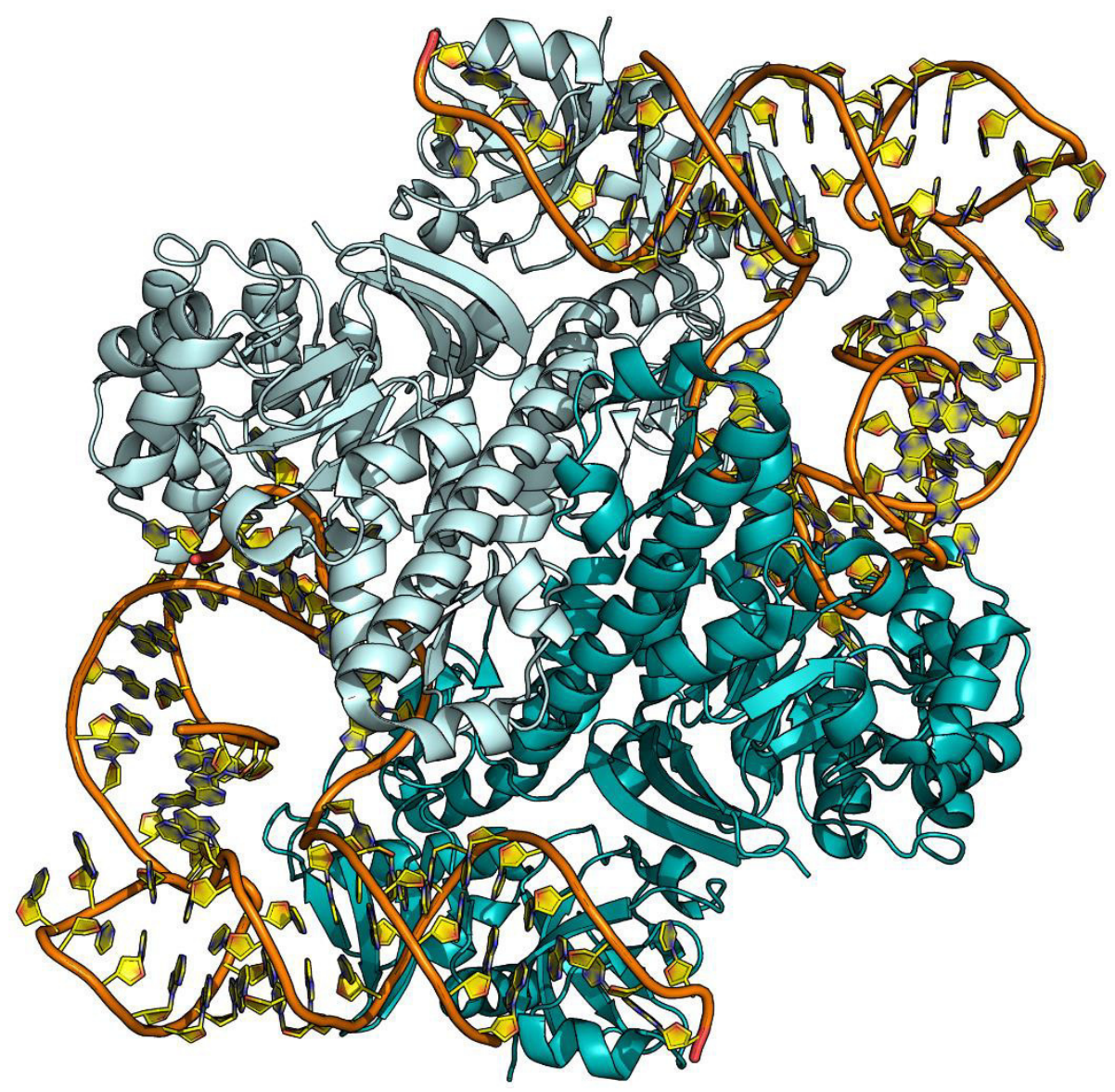

Figure 10. Crystal structure of the arcTGT tRNA complex.

The arcTGT homodimer from P. horikoshii (PDB-ID: 1J2B) is shown with individual coloring of both subunits (turquoise and light turquoise). A tRNA (orange/yellow) is bound to the active site of each monomer in a 1:1 ratio. The three $\mathrm{C}$-terminal domains of one subunit are engaged in extensive contact formation with the RNA, facilitating the insertion of the target base G15 in the active site of the respective other molecule. All components are depicted as cartoon. 


\subsubsection{THE EUKARYOTIC TGT}

In analogy to eubacteria, the G34 of the tRNAs tRNA ${ }^{\text {Asp }}$, tRNA ${ }^{\text {Asn }}$, tRNA ${ }^{\text {His }}$ and tRNA ${ }^{\text {Tyr }}$ is replaced by the modified base queuine in most eukaryotic organisms. But in contrast to bacteria, no biosynthetic pathway for queuine is known in eukaryotes. Therefore, these rely on scavenging this modified base from nutritional sources (153). The absence of a biosynthetic pathway and the more complex cellular organization of eukaryotic cells impose altered requirements on the TGT enzyme and indeed, the eukaryotic TGT exhibits striking structural differences to their bacterial counterparts. While bacterial TGTs are organized as a homodimer, the quaternary structure of the eukaryotic TGT is of heterodimeric organization. It is composed of a catalytical QTRT1 and a non-catalytical QTRT2 (or QTRTD1) subunit (154). This altered subunit organization, from a homomer in bacteria to a heteromeric assembly in eukaryotes, has been observed previously for a different tRNA modifying enzyme, the $\mathrm{m}^{1} \mathrm{~A} 58$ methyltransferase of Thermus thermophilus. This bacterial enzyme is a homotetramer, but in analogy to the bacTGT can only bind half the number of tRNA substrates, while the homologous enzyme from S. cerevisiae is organized as a homodimer of a heterodimer(155, 156).

QTRT1 and QTRT2 of the eukaryotic TGT are homologous to the eubacterial TGT exhibiting $42 \%$ sequence identity (157). Analysis of the sequence conservation found both subunits to harbor the three cysteines and a histidine which are required for coordination of the $\mathrm{Zn}^{2+}$ ion in the Z. mobilis TGT structure $(139,157)$. The catalytically essential residues identified in bacTGT are likely conserved in QTRT1. Asp279, the QTRT1 residue aligning to the catalytically important Asp280 in bacTGT, has been shown to be crucial for activity of the human TGT (154). In contrast to QTRT1, these amino acids are mutated to residues exhibiting substantially different chemical properties in the QTRT2 subunit $(141,157)$. Because of this observation, the QTRT2 subunit is generally considered to be deficient of a TGT related catalytic function (154). Despite its degeneration of the of the active site, QTRT2 does not only colocalize with QTRT1 in a cellular context (157), but its presence in the heterodimeric assembly is crucial for TGT activity in eukaryotes (154). Neither QTRT1- nor QTRT2-only samples do exhibit any transglycosylation activity which is exclusively observed in presence of the heterodimer $(141,154,157)$ highlighting the importance of QTRT2 for the enzymatic activity of the eukaryotic TGT. Investigations of the quaternary structure of the mammalian TGT by immunoprecipitation of lysate obtained from COS-7 cells, which were transiently transfected with the two TGT subunits, found both subunits to tightly interact. However, the 
authors were also able to identify a homodimer composition of QTRT2, while QTRT1 failed to self-associate ex vivo (157). Investigation of the recombinantly expressed and purified human TGT enzyme confirmed these observations of the quaternary structure of the subunits under in vitro conditions (154). Additionally, the authors observed a homodimerization not only of the QTRT2, but also of the catalytic QTRT1 subunit using intact mass analysis. An even more detailed analysis of the TGT quaternary assembly was conducted by Behrens et al. for the mouse enzyme (141). Mass-spectrometric analysis under non-denaturing conditions of either QTRT1 or QTRT2 in absence of the other respective subunit, allowed to quantify homodimerization. QTRT1 was found to weakly self-associate under the experimental conditions with only $20 \%$ of the molecules exhibiting a dimeric assembly, while $80 \%$ remained as a monomer in solution. Interestingly, this ratio was stable upon increased protein concentration. Investigation of the QTRT2 subunit alone revealed a much higher tendency of this subunit to self-associate, with $80 \%$ of the proteins forming dimers. Despite the high tendency of QTRT2 to be engaged in homodimeric assemblies, the addition of QTRT1 to the sample resulted in the large majority of molecules to form the heterodimeric TGT complex, with only a small minority of both subunits present as monomer or homodimer, confirming previous observations for the human and mouse TGT enzyme (141).

\section{6.3.1 STRUCTURE OF THE EUKARYOTIC TGT}

The recently reported crystal structure of the mouse QTRT2 subunit (PDB-ID: 6FV5) provids first structural insights into a eukaryotic TGT subunit and is shown in figure 11 (141). The overall fold and topology of the non-catalytic QTRT2 strongly resembles the structure of the bacterial monomer from $Z$. mobilis with a largely similar organization of the $(\beta / \alpha){ }_{8}$ barrel and the zinc ion being coordinated at the respective location. Investigation of the QTRT2 structure reveals the previously noted assumption that amino acids involved in the catalytic mechanism in the bacterial enzyme are not conserved in QTRT2, with the two catalytical residues Asp 280 and Asp102 from the Z. mobilis structure being replaced by a glutamate (Glu272) and a cysteine (Cys94) residue in the mouse QTRT2 subunit. Furthermore, the binding site for the modified base is degenerated with the $\alpha$-helix absent in QTRT2 that harbors the preQ $_{1}$ stacking residue Tyr106 in the bacTGT (PDB-ID: 1P0E). The QTRT2 structure furthermore shows a homodimeric assembly of two subunits, which is organized similarly to the homodimer of the Z. mobilis bacTGT (141). Because of the unknown function of this subunit outside of the TGT heterodimer, a biological consequence of this assembly remains elusive. 


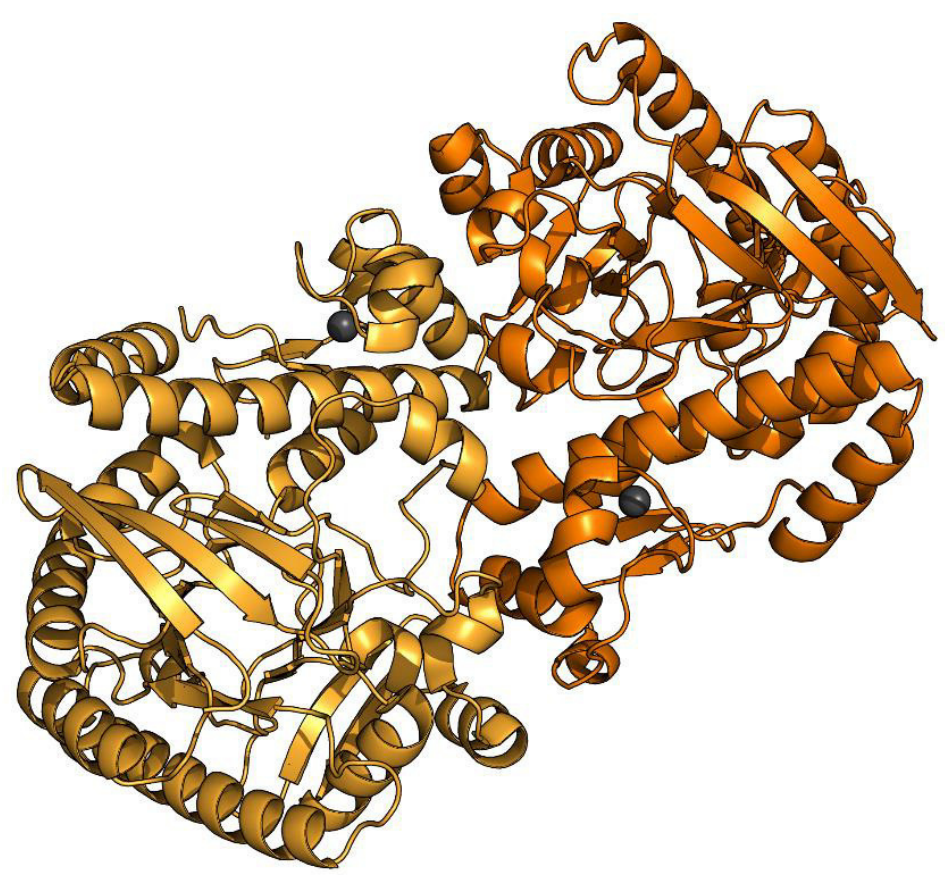

Figure 11. Crystal structure of the Mus musculus QTRT2 homodimeric assembly

The non-catalytic subunit, QTRT2, from mouse was crystallized as a homodimeric assembly (PDB-ID: 6FV5). Both subunits of the assembly depicted as cartoon, with individual coloring of the subunits. The zinc atom of each subunit it shown as grey sphere. The QTRT2 homodimer is organized similarly to the bacTGT homodimer, however no biological significance for this homodimer has been reported.

Do date no crystal structure of an eukaryotic TGT catalytic subunit has been reported. Hence, efforts were undertaken to provide structural insights into this subunit by deducting its structural organization from homology modeling based on the bacTGT structure from Z. mobilis as well as engineering of the bacTGT to mimic the eukaryotic TGT's active site (158). Indeed, the diffraction data of the bacTGT Tyr106Phe/C158Val/Val233Gly triple mutant does show electron density for the 7-deaza-guanine moiety of the co-crystallized queuine base but not for the cyclopentene ring. Furthermore, this mutant does not accept queuine as a substrate for the transglycosylation reaction (158), highlighting the necessity for a report of a QTRT1 structure.

\subsubsection{REGULATION OF THE EUKARYOTIC TGT}

Not only the uptake of queuine in eukaryotic cells, but also the activity of the TGT enzyme has been shown to be regulated. A study by Morris et al., investigating activity of the purified TGT derived from rat liver finds the enzyme to lose nearly all its activity over 4 days (159). This observation may not be surprising, as it is common knowledge that most enzymes are subject to activity loss over time when kept under artificial conditions. However, TGT activity can be 
restored to nearly $100 \%$ by adding a low amount of protein kinase C (PKC), which had also been implicated in queuine uptake in eukaryotic cells (1.5.3). The addition of phosphatases, such as alkaline phosphatase results in a contrary effect most likely caused by removal of phosphorylation. Transfer of ${ }^{32} \mathrm{P}$ by PKC and subsequent autoradiograph analysis of an SDSPAGE gel, loaded with the treated TGT protein, finds most radioactivity to be incorporated into the lower migrating band which, according to the authors, corresponds to the active subunit of the rat TGT heterodimer. Altogether, TGT activity and hence Q34tRNA levels seem to be positively regulated by PKC and its activity is counteracted by phosphorylases (159).

\subsection{BIOLOGICAL CONSEQUENCES OF QUEUINE MODIFICATION}

\subsubsection{Modulation of DnMt2 Dependent C38 Methylation}

The interplay of RNA modifications and how they affect each other is largely unknown. The presence of an average of 14 different modifications on a single tRNA molecule at the same time contributing to the tRNA's function rises the possibility that they may not only aid function of the tRNA but also could influence each other's abundance (160). Within their study, the authors who firstly identified Dnmt2 as a tRNA methyltransferase reported on the side that Dnmt2 dependent C38 methylation was strictly observed in concert with another modification (64). This modification was further investigated by mass spectrometry and identified as a mannosylated queuine which was incorporated at position 34 of the methylated tRNA. (64). Despite this observation the link between these two modifications was initially not further investigated by the field. A first hint that Dnmt2 activity might be altered by another factor came from the Ehrenhofer-Murray laboratory, who observed unexpectedly low C38 methylation of Dnmt2 target RNAs when $S$. pombe cells were grown in standard complete or minimal medium. On the other side, Dnmt 2 dependent methylation was much higher when the cells were grown under presence of peptone, which contains also several nutrients $(67,161)$. This nutrient was identified as the 7-deaza-guanine derivative queuine, a modified base that was supplemented to the cells as a component of the peptone mixture. Sole supplement of queuine to the cell medium had a drastic effect on Dnmt2 activity. In cells grown under absence of the free queuine base C38 modification of tRNA ${ }^{\text {Asp }}$ was low, with only $6 \%$ of the tRNAs modified, but when queuine was added to the medium, the tRNA ${ }^{\text {Asp }}$ pool became fully methylated. The authors could also show ex vivo that queuine modification of tRNA ${ }^{\text {Asp }}$ is 
responsible for this modulation of Dnmt2 activity (65). How exactly queuine mediates Dnmt2 stimulation on tRNA ${ }^{\text {Asp }}$ a molecular basis, and if other factors are involved in this effect is currently unclear.

\subsubsection{QUEUINE AND CELl PROLIFERATION}

The growth of human foreskin fibroblasts (HFF) in culture under constant presence of nanomolar concentration of the PKC activity-modulating compound phorbol-12,13didecanoate (PDD) results in a substantial decrease of Q34tRNA (162). Simultaneously, the constant addition of PDD results in a 5-10 fold increase of culture saturation density at early passages (163). Application of exogenous, purified queuine at $50 \mathrm{nM}$ concentration to the cells abolishes the PDD induced effect on maximal cell density and re-establishes cell growth similar to PDD untreated cells (162), suggesting that either the free queuine base or Q34 modified tRNA counteract the effect of the PDD. In contrast to these results, investigation of $\mathrm{rQT}_{3}$ transport in later-passage fibroblasts finds the $\mathrm{rQT}_{3}$ uptake to be stimulated by PDD. Simultaneously it was shown that the effect observed in the early-passage stage of HFF cells was caused by a cell produced factor that inhibits queuine uptake (164). The identity of this factor is not described in this study. However, other studies identified a $10-30 \mathrm{kDa}$ factor that was responsible for queuine inhibition (164) and finally led to the observation that interferons $\left(\alpha-, \beta\right.$ - and $\left.\gamma_{-}\right)$are responsible for the inhibition of queuine uptake (165). This led to the revised view that PKC activity is stimulating queuine uptake in the cells (124).

\subsubsection{QUEUINE AND CANCER}

Besides the observation that TGT is subject to regulation, queuine has been investigated in various tumor cells, where a lack of queuine at the wobble base position in the anticodon of TGT target tRNAs have been observed (166). Extraction of the tRNA pool and subsequent analysis identified various degrees of queuine hypomodification in tissues that show abnormal and excessive growth (neoplastic tissues) and transformed cells. While high queuine deficiency was observed in lung cancer (167), the degree of queuine hypomodification correlates with the severity of malignancy in several cancerous tissues in human, such as leukemia, lymphoma, brain and ovarian tumor as well as lung cancer (167-170). In particular, lack of tRNA queuine modification in high grade lymphomas is substantially more severe than in less malignant cancer types such as lymphocytic lymphoma at a favorable diagnostic stage (168). Evaluating 
the outcome of their study, the authors highlight the importance of queuosine for disease activity and imply its role in maturation of certain types of cancer.

The apparent strong correlation between a lack of Q34tRNAs and the proliferation state of the malignant tissue, raises the idea that the lack of queuine modified tRNA may be simply due to the lack of free queuine in the cell, a consequence of limited supply caused by the abnormal fast growth of the respective tissue. This theory is supported by Osaka et al., who measured the amount of queuine incorporated into tRNA by quantifying the amount of radioactively labelled guanine incorporated by the $E$. coli TGT enzyme. Results indicate queuine hypomodification to be closely related to the state of cell differentiation (171). Analysis of mitochondrial tRNA from Morris hepatoma cells highlights a defective queuosine incorporation into tRNA ${ }^{\text {Asp }}$ at an early stage of cancer development, involving abnormalities in mitochondria. The authors hypothesize that queuine hypomodification is caused either by a deficient uptake of queuine or inhibition of the TGT enzyme. Indeed, TGT activity as well as queuine uptake into the cell have been found to depend on activity of $\operatorname{PKC}(159,162)$, which is involved in cell proliferation and tumor genesis and is a prominent receptor for cancerogenic phorbol esters (172). A hint that queuine hypermodification may indeed be caused substrate limitation came with the observation that the administration of external queuine to cancerous mice results in an increased level of Q-modified tRNA in cancerous tissues and coincidentally causes the inhibition of tumor growth (173). However, administration of higher queuine levels might also affect activity of a potentially inhibited TGT enzyme. In support of this, Emmerich et al. found that queuine hypomodification might not be caused by lack of queuine as they observed similar free queuine concentrations in neoplastic and non-neoplastic tissues of about $100 \mathrm{nM}$, a concentration that is sufficient to allow full queuine modification of the tRNA pool $(119,168)$. Whether modulation of PKC activity during tumor genesis is the causative factor and which step of Qmodification is hindered, has not been shown. Furthermore, characteristic features of malignant tumor growth, such as higher turnover of tRNAs and excretion of modified nucleosides, might contribute to hypomodification of the respective tRNAs with queuine $(174,175)$. 


\section{CHAPTER 2: STRUCTURAL ANALYSIS OF QTRT1}

This manuscript was originally published in Biomolecules.

\section{Crystal Structure of the Human tRNA Guanine Transglycosylase Catalytic Subunit QTRT1}

Sven Johannsson, Piotr Neumann and Ralf Ficner*

\section{Author affiliations:}

Department of Molecular Structural Biology, Institute of Microbiology and Genetics, GZMB, Georg-August-University Göttingen, 37077 Göttingen, Germany; sjohann1 @ gwdg.de (S.J.); pneuman2@gwdg.de (P.N.)

* $\quad$ Correspondence: rficner@uni-goettingen.de; Tel.: +49-551-3914072

Keywords: TGT; QTRT1; queuine; tRNA modification; wobble base

Biomolecules 2018, 8(3), 81

DOI: 10.3390/biom8030081

Received: 24 July 2018; Accepted: 20 August 2018; Published: 24 August 2018 


\section{Author contributions:}

S.J. and R.F. designed the experiments. S.J. performed cloning and purification of proteins. Crystallization and sample preparation were done by S.J. S.J. also performed initial molecular replacement. PN did the MR-SAD experiment and respective data interpretation. Building of the final structures was performed by S.J. The structures were interpreted by S.J., P.N. and R.F.. S.J., P.N., and R.F. prepared the manuscript. 
Article

\title{
Crystal Structure of the Human tRNA Guanine Transglycosylase Catalytic Subunit QTRT1
}

\author{
Sven Johannsson, Piotr Neumann and Ralf Ficner * \\ Department of Molecular Structural Biology, Institute of Microbiology and Genetics, GZMB, \\ Georg-August-University Göttingen, 37077 Göttingen, Germany; sjohann1@gwdg.de (S.J.); \\ pneuman2@gwdg.de (P.N.) \\ * Correspondence: rficner@uni-goettingen.de; Tel.: +49-551-3914072
}

Received: 24 July 2018; Accepted: 20 August 2018; Published: 24 August 2018

check for updates

\begin{abstract}
RNA modifications have been implicated in diverse and important roles in all kingdoms of life with over 100 of them present on tRNAs. A prominent modification at the wobble base of four tRNAs is the 7-deaza-guanine derivative queuine which substitutes the guanine at position 34 . This exchange is catalyzed by members of the enzyme class of tRNA guanine transglycosylases (TGTs). These enzymes incorporate guanine substituents into tRNA ${ }^{\text {Asp }}$, $\mathrm{tRNA}^{\mathrm{Asn}} \mathrm{tRNA}^{\mathrm{His}}$, and tRNA ${ }^{\mathrm{Tyr}}$ in all kingdoms of life. In contrast to the homodimeric bacterial TGT, the active eukaryotic TGT is a heterodimer in solution, comprised of a catalytic QTRT1 subunit and a noncatalytic QTRT2 subunit. Bacterial TGT enzymes, that incorporate a queuine precursor, have been identified or proposed as virulence factors for infections by pathogens in humans and therefore are valuable targets for drug design. To date no structure of a eukaryotic catalytic subunit is reported, and differences to its bacterial counterpart have to be deducted from sequence analysis and models. Here we report the first crystal structure of a eukaryotic QTRT1 subunit and compare it to known structures of the bacterial TGT and murine QTRT2. Furthermore, we were able to determine the crystal structure of QTRT1 in complex with the queuine substrate.
\end{abstract}

Keywords: TGT; QTRT1; queuine; tRNA modification; wobble base

\section{Introduction}

RNA molecules have been shown to undergo extensive post-translational modification with 171 modifications known to date and over 100 of them occurring on transfer RNAs (tRNAs) [1,2]. In tRNA these modifications fulfill diverse functions, ranging from their influence on the three-dimensional (3D) structure to tuning of interactions with proteins. Incorporation of modifications into tRNA is also a dynamic process, as their distribution, as well as abundance level, might change upon nutrient alteration and stress changes [3]. The function of a particular modification is not only determined by its chemical properties but also highly dependent on its location in the tRNA molecule. While modifications in the structurally important D-loop and T-loop regions may alter and fine tune the tRNA's three dimensional shape [4], modifications harbored in the anticodon stem loop are implicated to modify codon-anticodon interaction and/or are relevant during protein translation [5].

A prominent modification in the anticodon loop, which is present in the majority of bacterial and eukaryotic organisms, is the 7-deaza-guanine derivative queuine, which substitutes the nucleic base guanine at the wobble base position 34 of four tRNAs: tRNA ${ }^{\text {Asp }}$, tRNA ${ }^{\text {Asn }}, \mathrm{tRNA}^{\mathrm{His}}$, and tRNA ${ }^{\mathrm{Tyr}}$ [6]. In contrast to eukaryotes, bacteria synthesize queuine de novo. Starting from guanosine $5^{\prime}$-triphosphate the queuine precursor 7-(aminomethyl)-7-deazaguanine (preQ1) is built up outside the tRNA [7-13]. The incorporation of $\mathrm{preQ}_{1}$ into the tRNA is catalyzed by the enzyme tRNA-guanine transglycosylase (TGT) in a proposed ping-pong mechanism [14]. The 3D structure of a eubacterial TGT was firstly 
described by Romier et al. for the Zymomonas mobilis enzyme, a $43 \mathrm{kDa}$ protein. This eubacterial TGT (bacTGT) harbors an eight stranded beta $(\beta / \alpha)_{8}$ triose-phosphate-isomerase like fold, a $(\beta / \alpha)_{8}$ TIM barrel, which is a characteristic of this enzyme class that provides the structural basis for substrate binding and catalysis [15]. Furthermore, it contains a zinc ion binding site which, in concert with the TIM barrel, provides the scaffold for binding of the tRNA substrate by this enzyme class [16-18].

Due to the scarce structural knowledge, insights into tRNA recognition are mainly provided by the structure of the archeosine Pyrococcus horikoshii TGT (arcTGT) in complex with a full length tRNA. However, arcTGT specifically targets the G15 base of cognate tRNAs for modification, rather than G34 like the eubacterial and eukaryotic enzymes [18-20]. Insights into the tRNA protein interaction and the underlying mechanism of the transglycosylation reaction for the bacterial enzyme were provided by the crystal structure of the covalently bound tRNA anticodon stem loop to bacTGT from Z. mobilis. Interestingly, this complex structure revealed Asp280 to execute the nucleophilic attack on the $C$ atom of the $\mathrm{N}$-glycosidic bond between the ribose and the guanine base rather than Asp102, the residue that was originally proposed to be involved in this cleavage $[15,21]$. The consequence of this nucleophilic attack is the formation of a covalent tRNA-enzyme reaction intermediate in which RNA and protein are linked by a covalent bond between the Asp280 and the remaining ribose of the tRNA backbone [15]. The cleaved guanine base is free to dissociate from the active site and is subsequently replaced by the preQ1 substrate. Formation of the $\mathrm{N}$-glycosidic linkage between the tRNA and preQ1 is then achieved by deprotonation of the preQ1's N5 atom through the TGT's Asp102, which facilitates a nucleophilic attack of preQ1 on the covalent tRNA Asp280 intermediate leading to formation of the final reaction product. Finally, the modified tRNA product dissociates from the enzyme in a rate limiting step [22].

This mechanism of queuine incorporation by bacterial TGTs has recently become of interest for drug design as TGT has been identified as an important virulence factor. In Shigella, a gram negative bacterium and the causative factor of shigellosis in human, the absence of a functional TGT strongly reduces pathogenicity, allegedly caused by queuine hypomodification of the mRNA encoding the transcriptional factor virF [23-25]. Therefore the bacterial TGT has emerged as a valuable target for structure based inhibitor design [26,27]. Beside infection by Shigella, queuine has also been proposed to promote host infection of the pathogenic ameba Entamoeba histolytica, which might scavenge the queuine base from gut bacteria [28].

As for E. histolytica, most eukaryotes possess TGT enzymes, but, in contrast to their bacterial counterparts, they do not encode for enzymes necessary to build up queuine and therefore have to incorporate the queuine base, instead of a precursor, into RNA targets. The essence of queuine in eukaryotes requires humans and other organisms to scavenge this modified base from nutritional sources and/or from microbiomes [29,30]. Likely to meet these altered demand and more complex pathways and regulation in eukaryotic cells, properties of eukaryotic TGTs have emerged to show significant differences to their bacterial counterparts. In contrast to the homodimeric architecture of the bacterial and archeal TGT, the eukaryotic, heterodimeric TGT is composed of a catalytically active queuine tRNA-ribosyltransferase subunit 1 (QTRT1) and a catalytically inactive QTRT2 subunit. This quaternary structure has been confirmed to be essential for catalyzing the incorporation of queuine into tRNA [31]. Recently, the crystal structure of the noncatalytic mouse QTRT2 subunit has been solved and found to confirm the prior suggestion that this subunit is incapable to catalyze the guanine to queuine exchange reaction as catalytic residues, and the residues involved in substrate coordination are largely replaced by chemically different amino acids and thus are extremely unlikely to facilitate queuine binding or even the transfer reaction [32]. In contrast, alignment of the human QTRT1 sequence with the Z. mobilis TGT reveals this subunit to be likely catalytically active with relevant residues in place. However, despite the potential importance of this subunit as a drug target, its structure remains unknown.

Understanding the mechanism of queuine incorporation by the eukaryotic TGT enzyme is of great interest as altered presence of queuine in TGT substrates has been associated with various disease related effects such as cancer. Here queuine hypomodification in tRNA has been linked to 
increased cancer progression $[33,34]$ as well as impaired cell differentiation [35]. Furthermore, mice which were genetically modified to lack a functional TGT enzyme showed symptoms similar to those associated with the disease polyphenylketonurea as they were deficient in producing tyrosine from phenylalanine [36]. The underlying mechanism of this effect is not known to date, but Rakovich et al. were able to link this observation to absence of QTRT1 and consequently to the lack of queuine in the tRNA pool.

Additionally, queuine has been proposed to increase stress tolerance [37], an effect unraveled more than 20 years ago of which the mechanistic details were only recently described. Here, queuine modification of tRNA ${ }^{\text {Asp }}$ has been proven in vivo and in vitro to trigger activity of the methyltransferase Dnmt2 which results in establishment of $\mathrm{m}^{5} \mathrm{C}$ modification at cytosine $38\left(\mathrm{~m}^{5} \mathrm{C} 38\right)$ of this tRNA $[38,39]$.

Despite the numerous implications of TGTs in diseases in mammals, little is known on the structural level of queuine incorporation by eukaryotic TGT enzymes, as information about the catalyzed reaction was deducted from analysis of bacterial TGT structures through homology modeling. Recently, the QTRT2 crystal structure allowed the first structural insights into the arrangement of the noncatalytic subunit and potential dimer assembly, leaving the biological significance without a functional catalytic site unsolved. The 3D structure of a eukaryotic TGT catalytic subunit, which would provide reliable information about the architecture of the active site and thus the mechanism, is not known.

Here we report the crystal structure of the human QTRT1 subunit in its apo form and in complex with the queuine base. The performed analysis reveals the QTRT1 fold to be highly related to the bacterial TGT of Z. mobilis while the active site is altered in order to accommodate queuine base, which is bulkier than preQ1. Furthermore, comparison with the QTRT2 crystal structure provides evidence that this noncatalytic subunit is unlikely to harbor the ability of binding queuine in the active center.

\section{Materials and Methods}

\subsection{Cloning of Human TGT}

QTRT1 and QTRT2 were ordered as synthetic genes from Invitrogen GeneArt (ThermoFisher Scientific, Waltham, MA, USA) as codon optimized sequences for expression in E. coli. QTRT2 and QTRT1 were cloned sequentially into the pCDF Duett 1 vector using the restriction enzymes NdeI/KpnI and EcoRI/HindIII, respectively, with QTRT1 harboring a N-terminal PreScission site for proteolytic cleavage of the 6 xHis tag.

\subsection{Expression and Purification}

The human TGT heterodimer QTRT1 and QTRT2 were co-expressed from pCDF-Duett vector with a cleavable N-terminal 6xHis tag to QTRT1 (his-hTGT) in Escherichia coli BL21(DE3)-STAR cells using autoinduction. The medium was supplemented with additional $100 \mu \mathrm{M} \mathrm{ZnCl}_{2}$. Cells were grown at $18{ }^{\circ} \mathrm{C}$ for $50 \mathrm{~h}$ before harvesting. Cell pellets were flash-frozen in liquid $\mathrm{N}_{2}$ and stored at $-80{ }^{\circ} \mathrm{C}$ until further use. For purification purposes the cells were thawed and disrupted by microfluidization (M-110S Microfluidizer (Microfluidics, Westwood, MA, USA)) (50 mM HEPES $\mathrm{pH} 7.5,100 \mathrm{mM} \mathrm{NaCl}, 10 \mathrm{mM}$ imidazole). Soluble protein was isolated by ultracentrifugation at 50,000 g for $30 \mathrm{~min}$. His-hTGT was loaded onto His-Talon Superflow (GE Healthcare, Chicago, IL, USA) and washed with additional $1 \mathrm{M} \mathrm{LiCl}$. Protein was eluted in a $20 \mathrm{CV}$ gradient to $50 \mathrm{mM}$ HEPES pH7.5, $100 \mathrm{mM} \mathrm{NaCl}, 500 \mathrm{mM}$ imidazole. Target protein containing fractions were pooled and supplemented with PreScission protease (1:100 w/w) and incubated for 16-18 h under mild agitation. The protein was further purified by Superdex S200 (GE Healthcare) size exclusion chromatography (20 mM HEPES pH 7.5, $100 \mathrm{mM} \mathrm{NaCl}$ ). hTGT was concentrated to $6-8 \mathrm{mg} / \mathrm{mL}$ and further processed, or flash frozen in liquid $\mathrm{N}_{2}$ and stored at $-80^{\circ} \mathrm{C}$ until further use. 


\subsection{Crystallization}

QTRT1 was crystallized using sitting-drop or hanging drop vapor diffusion technique with protein concentration adjusted to $6 \mathrm{mg} / \mathrm{mL}$ as determined with Bradford reagent (Bio-Rad, Hercules, CA, USA). Equal volumes of protein containing solution and crystallization condition (100 mM Tris pH 7.8, 200 mM KBr, 200 mM KSCN, 3\% (w/v) $\gamma$-polyglutamic acid-LM (PGA-LM), 5\% PEG 4000) were mixed. Crystallization was performed in EasyXtal-15-well tool plates (Qiagen, Hilden, Germany) with the cap loosened at a $\frac{1}{4}$ turn to allow for evaporation or in loosely sealed 24 well Cryschem plates (Hampton Research, Aliso Viejo, CA, USA). Crystals were obtained after five to seven days at $20^{\circ} \mathrm{C}$ from crystallization drops of $0.5-6 \mu \mathrm{L}$ total volume. To obtain QTRT1 in complex with queuine, respective crystals were soaked in the crystallization condition containing the free queuine base at $50 \mu \mathrm{M}$ concentration. Crystals were cryo-protected by a stepwise increase of glycerol and PEG400 concentration to $15 \%(v / v)$ each.

\subsection{Data Collection, Molecular Replacement and Refinement}

X-ray data collection was performed at $-173^{\circ} \mathrm{C}$; diffraction images were collected at P14 beamline, operated by EMBL Hamburg, at the PETRA III storage ring (DESY, Hamburg, Germany). Diffraction images were indexed, integrated, and scaled using the XDS-package [40]. The structure of QTRT1 was solved by molecular replacement by PHASER [41], as implemented in the CCP4 suite [42]. The crystal structure of the Zymomonas mobilis tRNA guanine transglycosylase [16] (PBD-ID: 1PUD) was used as a model and modified with CHAINSAW, as implemented in the CCP4 suite. MR-SAD was performed using the phenix.autosol pipeline. To overcome the difficulties in manual model rebuilding, both QTRT1 monomers were subjected to energy and density-guided refinement in Rosetta [43] utilizing constrains from the MR-SAD electron density map. Model building was done with subsequent iterative cycles of automated refinement with PHENIX [44] and manual model adjustments in Coot [45]. Crystallographic values are listed in the Appendix A.

\subsection{Q-Base Synthesis}

The queuine base was synthesized as described previously, starting from inexpensive methyl $\alpha$-D-galactopyranoside for the (1R,2S,3S)-1-bromo-2,3-O-isopropylidene-cyclopent-4-ene building block and preQ1 [38].

\section{Results}

To obtain the structure of the human TGT, we cloned the coding sequences for both human TGT subunits, QTRT1 and QTRT2, into a pCDF Duett vector and co-expressed both full length constructs in E. coli BL21 DE3 cells. The heterodimer was purified with subsequent affinity purification, cleavage of the 6xHis tag from the QTRT1 subunit and size exclusion chromatography, yielding a pure, nucleotide free, and active TGT heterodimer. The purified TGT heterodimer was submitted to crystallization process immediately after purification. The protein containing solution was mixed with the crystallization condition ( $100 \mathrm{mM}$ Tris pH 7.8, $200 \mathrm{mM} \mathrm{KBr}, 200 \mathrm{mM} \mathrm{KSCN}, 3 \%(w / v)$ PGA-LM and 5\% PEG 4000) in a 1:1 ratio. Crystals were obtained after 5 to 14 days as plates with varying sizes of 40 to $150 \mu \mathrm{m}$ utilizing sitting and hanging drop experimental setups. Crystallization was favored by slowly increasing the concentration of components in the drop with controlled evaporation. The crystals were cryoprotected and exposed to synchrotron radiation. Multiple diffraction images datasets of the best diffracting crystal were collected, processed with XDS and subsequently merged with a resolution cutoff at $2.45 \AA$. The space group was identified to be $\mathrm{C} 2$. We were able to obtain initial phases by molecular replacement using the crystal structure of the Z. mobilis TGT monomer (Protein Data Bank (PDB)-ID: 1PUD) [16]. The crystal's asymmetric unit comprises two molecules with only one of them (chain A) exhibiting reasonable fit to electron density after initial refinement steps. The other molecule (chain B) only marginally fit the electron density and no interpretable 
density was observed for about 160 of the N-terminal amino acids. For validation of the molecular replacement (MR) model we used the initial phases from the MR and performed MR-SAD utilizing the weak anomalous signal of the merged dataset following by model rebuilding with Rosetta software. MR-SAD confirmed location of the zinc ion in both molecules occupying the asymmetric unit and improved phases which allowed to build both molecules simultaneously, thus advancing productivity during the model building and refinement process.

The purified TGT used for protein crystallization consisted of both subunits as confirmed by SDS-PAGE and was catalytically active, as shown by Q-incorporation into G34tRNA Asp [38]. However, structural analysis of the asymmetric unit's content revealed both molecules to be the catalytic QTRT1 subunit.

The structure consisting of two QTRT1 subunits (PDB-ID: 6H42) was refined to a final $\mathrm{R}_{\text {work }}$ and $R_{\text {free }}$ of 20.13 and $23.96 \%$ respectively, with the first subunit (chain A) exhibiting B-factors in the mid $60 \AA^{2}$ while chain B appeared to be more flexible within the crystal lattice with an averaged B-factor of around $70 \AA^{2}$. However, analysis of both molecules with respect to folding revealed no significant discrepancies between them. Analysis of the human QTRT1 catalytic subunit reveals the enzyme to possess a bended $(\alpha / \beta)$ barrel, consisting of an eight stranded $\beta$-barrel at the core and eight flanking helices (Figure 1). In addition, the protein contains a $\mathrm{Zn}^{2+}$ binding domain, in which the zinc ion is coordinated by a triangular arrangement of three cysteine residues Cys317, Cys319, and Cys322, while the $\alpha$-helix bound His 248 completes the tetragonal coordination of the metal ion. A detailed listing of data processing and refinement statistics can be found in the crystallographic table (Appendix A). Furthermore, an alignment of the sequences from the human QTRT1, Z. mobilis TGT and mouse QTRT2 is presented in Appendix B.

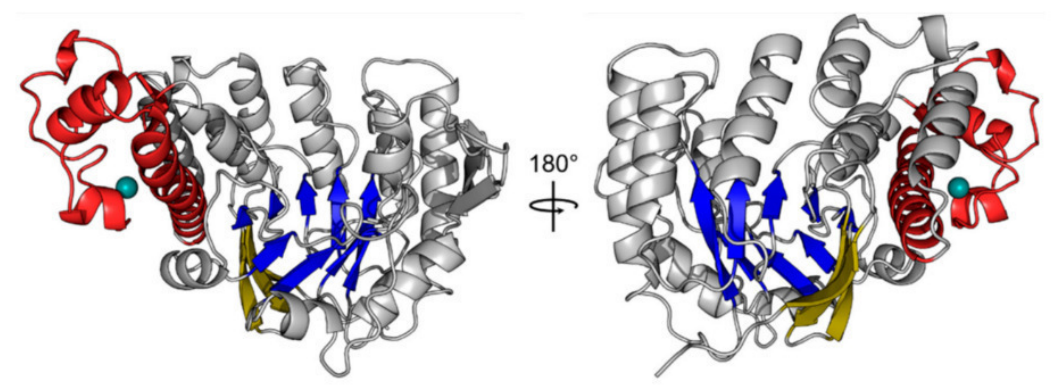

Figure 1. Crystal structure of the human tRNA guanine transglycosylase (TGT) catalytic subunit queuine tRNA-ribosyltransferase (QTRT1). The crystal structure of the QTRT1 monomer (PDB-ID: $6 \mathrm{H} 42)$ is presented as cartoon. The eight $\beta$-sheets of the $(\alpha / \beta)_{8}$ barrel at the core of the protein are shown in blue with a three stranded $\beta$-sheet acting as a lid (yellow) which covers the bottom of the barrel. QTRT1 additionally harbors a zinc binding domain (red), which tetragonally coordinates a zinc-ion (turquoise). Both structures show the same molecule rotated $180^{\circ}$ around the $y$-axis.

Comparison of the human QTRT1 structure with the previously reported structure of its bacterial counterpart from Z. mobilis reveals both proteins to share a fairly similar fold with a root-mean-square deviation (RMSD) of $2.86 \AA$ (calculated between all equivalent C $\alpha$ positions). Especially the arrangement of the $(\alpha / \beta)_{8}$ barrel and the zinc ion binding domain in this protein seems to be structurally conserved from bacteria to eukaryotes. Differences between both structures were observed in the conformation of the region comprising amino acids 114-138 which adopts a three stranded beta sheet with an extended loop between the $\beta$-strands two and three that folds into a more open conformation compared to the bacterial TGT. Furthermore, we did observe electron density for an additional C-terminal $\alpha$-helix. Most significant differences are observed in regions lacking a defined secondary structure. In the bacTGT enzyme two aspartate residues have been identified as catalytically active, with Asp280 performing the initial nucleophilic attack and the nucleophilic Asp102 sidechain supporting the formation of the reaction product. In the reported 
QTRT1 structure we observed conserved Asp105 and Asp279, occupying structurally equivalent positions, thus arguing for a conserved catalytic mechanism (Figure 2). As a consequence of the purification strategy, which included a high salt concentration, we did not observe additional electron density in the active site that may correspond to a bound ligand.

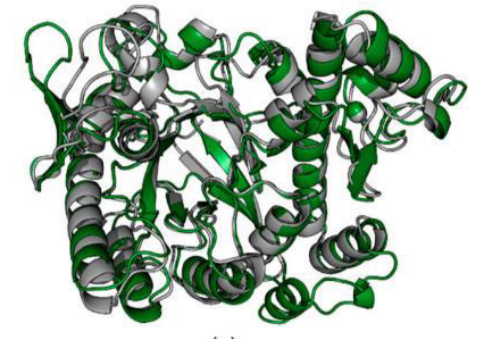

(a)

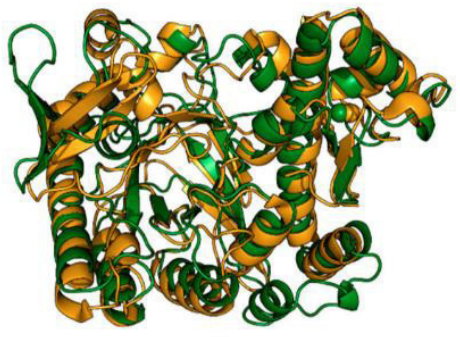

(b)

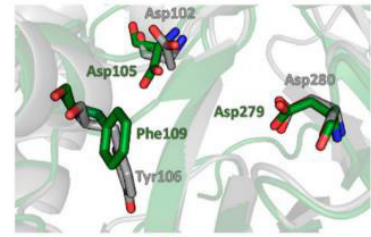

(c)

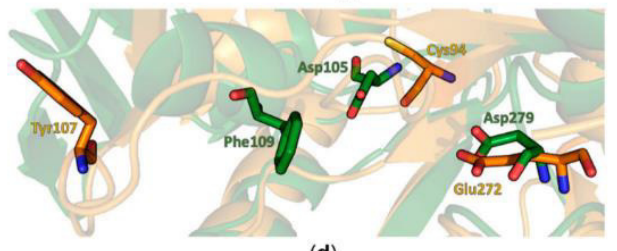

(d)

Figure 2. Structural comparison of QTRT1 to bacTGT and QTRT2. (a) Overlay of the human QTRT1 structure (green) with the bacterial TGT monomer of Zymomonas mobilis in grey (PDB-ID: 1PUD). Both structures are depicted as cartoons. The zinc ion is presented as a sphere in the respective color of the structure. (b) Alignment of the human QTRT1 structure (green) to the monomer structure of mouse TGT noncatalytic subunit QTRT2 (orange). Both structures are presented as cartoons with the respective zinc ions depicted as spheres. (c) Zoom into the active site of the overlay shown in (a) with according coloring of the molecules. bacTGT active residues Asp102 and Asp280 as well as the preQ1 stacking Tyr106 are depicted as grey sticks. Equivalent residues in QTRT1 are presented as green sticks. (d) Zoomed in view into the QTRT1 active site. QTRT1 residues presented in (c) are depicted in green. The respective residues in QTRT2 (orange) were identified upon alignment of the two protein sequences. The QTRT1 Phe109 aligned QTRT2 residue Tyr107 is displaced in the QTRT2 structure.

Comparison of the reported structure of the human QTR1 with the crystal structure of the noncatalytic QTRT2 subunit from mouse unveiled more striking differences. While the beta barrel and the zinc ion binding domain colocalize well in an alignment of both monomers (Figure 2b), more substantial differences could be observed in the active core. The catalytically active Asp279 of QTRT1 is exchanged by a chemically similar but more spacious glutamate in QTRT2 (Glu272) and Asp105 of QTRT1 is replaced by a chemically distinct cysteine residue (Cys94). The Phe109 residue, which is structurally equivalent to the bacTGT Tyr106 in QTRT1 (Figure1b), is replaced by a tyrosine in QTR2 (Tyr107). As a consequence of an altered conformation of the underlying polypeptide chain, Tyr107 is displaced from the active site in QTRT2. Comparison of the residues flanking the active site reveals striking differences between QTRT2 and both QTRT1 and bacTGT. In QTRT1 amino acids 108 to 114 adopt a $\alpha$-helical conformation that consecutively results in placement of Phe109 in close proximity to the active aspartate residues, an arrangement highly similar to the respective residues in bacTGT. This $\alpha$-helix is likely not to obstruct substrate binding as the active site is still accessible for substrates. Therefore, we refer to this structural arrangement as the "competent" conformation. In contrast, the respective stretch of amino acids in QTRT2 adopts a strikingly different conformation. Instead of formation of the mentioned helix, the peptide chain protrudes into the degenerated active site as a loop. This conformation is stabilized by stacking formed between the Try116 and Pro104 side chains and results in the mentioned displacement of Tyr107. We refer to this arrangement as the "incompetent" conformation, as is very likely would interfere with substrate binding. 
Binding of the bacterial TGT to its substrate preQ1 has previously been proposed as a valuable target for drug design [46]. A dilemma for structure based drug development has been the unknown structural differences of the bacterial and eukaryotic TGT active site, as avoiding cross-targeting of the human TGT is one of the main challenges in small molecule drug development. Efforts with structural prediction, alignments and even engineering of the bacterial TGT's active site to mimic its eukaryotic counterpart through amino acid exchanges have been undertaken to tackle this problem, but are still not the ultimate proof [47].

We were able to solve the crystal structure of the QTRT1 subunit in complex with queuine (PDB-ID: 6H45), by soaking the fully modified base into QTRT1 crystals. As a consequence of soaking, we did observe additional electron density corresponding to queuine in the active site (Figure 3), that clearly is absent in the apo QTRT1 structure. Binding of the queuine 7-deaza-purine moiety is promoted by stacking interactions and the shape of this density corresponds well to the queuine chemical structure. In addition, the excess electron density map localizes at the site which is equivalent to the preQ1 binding site in the bacterial TGT enzyme. The 7-deaza-guanine moiety is stacking between Phe109 and Met 259 side chains which have previously been implicated in queuine binding in eukaryotic TGTs. Furthermore, correct positioning of the queuine base for the enzymatic reaction is largely provided through polar contacts formed with Asp159. The exocyclic oxygen forms contacts with the polypeptide mainchain at residue Gly229, thus completing the coordination of the 7-deaza-purine. We also did observe density in the $\mathrm{mF}_{\mathrm{o}}-\mathrm{DF}_{\mathrm{c}}$ omit map for the additional cyclopentene-cis-diol, a hallmark of this modification, which distinguishes queuine from the bacTGT substrate $\mathrm{preQ}_{1}$. The di-hydroxy-cyclopentene moiety is located in a grove of the protein surface, distant from the QTRT1 catalytic residues and thus also to the anticipated binding site of the RNA substrate. While the space to accommodate the cyclopentene ring is provided by the Gly232 and Gly233 residues, its coordination is achieved by polar contacts of the cyclopentene C5 hydroxy group and Ser164 of the protein chain. Compared to the QTRT1 apo structure the conformational changes induced upon queuine binding are rather minimal. The QTRT1 main chain and side chains involved in stacking or polar contacts formation with queuine exhibit a fairly similar conformation in both, the complex and the apo structure. The most striking difference is observed for Ser 231 which is shifted away from the queuine ligand presumably to provide necessary space for accommodation of the cyclopentene moiety.

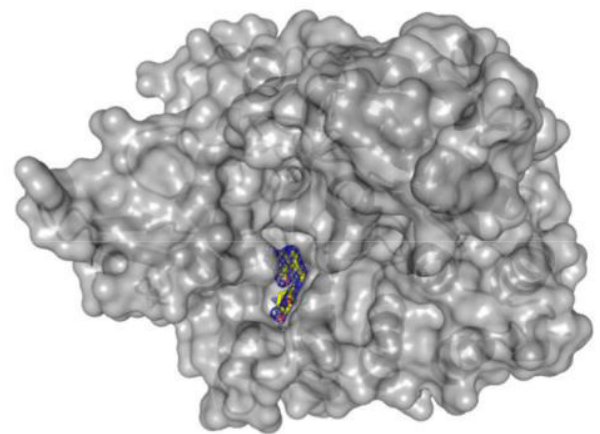

(a)

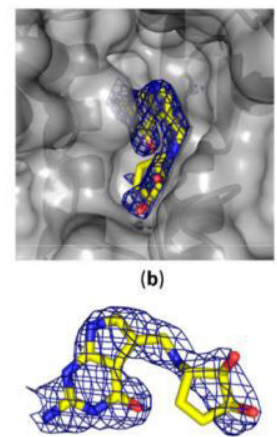

(c)

Figure 3. Crystal structure of human QTRT1 in complex with queuine. (a) Crystal structure of the human QTRT1 subunit in complex with queuine (PDB-ID: 6H45). QTRT1 is depicted in grey with a partly transparent surface and underlying polypeptide chain presented as a cartoon. Queuine is presented in yellow as sticks with blue nitrogen atoms and oxygens in red. $\mathrm{DF}_{0}-\mathrm{mF}_{\mathrm{c}}$ map for the omitted queuine base is shown as blue mesh with $2.0 \sigma$ contour level. (b) Close-up view into the active site with the complexed queuine base. (c) Conformation of the queuine base bound to QTRT1. Blue electron density shows $\mathrm{DF}_{0}-\mathrm{mF}_{\mathrm{c}}$ omit map contoured at $2.0 \sigma$. 


\section{Discussion}

To date structural investigation of tRNA guanine transglycosylases mainly focused on the bacterial TGT of Z. mobilis and the archeal TGT from Pyrococcus horikoshi. While these enzymes catalyze an incorporation of the modified bases preQ1 at position 34 and preQ0 at position 15 into cognate tRNAs respectively, the structure of the eukaryotic TGT that incorporates the finally modified queuine base was unknown. Here we report the first crystal structure of the human QTRT1, which is the catalytic subunit of a eukaryotic TGT enzyme.

The QTRT1 crystal structure resembles the previously reported two hallmarks of TGTs, namely the $(\alpha / \beta)_{8}$ barrel that builds the supporting structure for the catalytic center, and a zinc binding domain. We find these two structural motifs to be organized in a fairly similar manner as was observed in the previously reported structures of the eubacterial Z. mobilis TGT and the noncatalytic mouse QTRT2 protein.

Furthermore, we were able to obtain the structure of human QTRT1 in complex with queuine. Presence of the bound substrate has been confirmed by the calculated $\mathrm{mF}_{0}-\mathrm{DF}_{\mathrm{c}}$ map (Figure 3). Queuine localizes at the proposed binding site which corresponds to the preQ1 binding site in bacTGT. We tried also to obtain crystals with queuine present in the crystallization condition, but were unsuccessful to obtain crystals of suitable quality. Therefore, we chose to soak the ligand into apo crystals. We like to note that soaking vs. co-crystallization has the disadvantage that conformational changes upon ligand binding might not be accounted for, which might result in ligand positional deviations between the soaked and the co-crystallized structure. However, we find the queuine base in our complex structure is consistent with results from a previous study by Biela et al., that aimed to enable the bacTGT enzyme to bind the queuine base [47]. The authors identified residues that may allow for binding of queuine in the human QTRT1 by homology modeling. They were able to observe electron density for the queuine's dicyclic purine when they introduced Tyr106Phe and Cys158Val mutations in bacTGT to mimic the queuine binding site of the human TGT. However, they were not able to locate the cyclopentene moiety. Our QTRT1 queine complex structure confirms their observation that the human QTRT1 Phe109 (the human equivalent of Try106 in bacTGT) stabilizes queuine binding through stacking with the purine part of the molecule. We can also confirm that indeed the bacTGT's Cys158 residue colocalizes with Val161 of the human QTRT1, when both structures are aligned. Furthermore, we observe that absence of a sidechain at position 232 and 233 in QTRT1 does allow the accommodation of the bulky cyclopentene-diol ring which would otherwise be obstructed. This is not the case in the bacTGT enzyme, where instead of Gly232, a valine (Val233) is present at this location, that would interfere with binding of the cyclopentene.

A structural alignment of the QTRT1 structure with the structure of the bacTGT solved in complex with an RNA substrate reveals Asp105 and Asp279 occupying the same positions as the respective catalytic residues in the catalytic bacTGT enzyme (Figure 4). The observation that queuine is bound by QTRT1 at the respective site where preQ1 is bound in the bacTGT enzyme argues for a conserved catalytic mechanism, although the substrate base is different. 


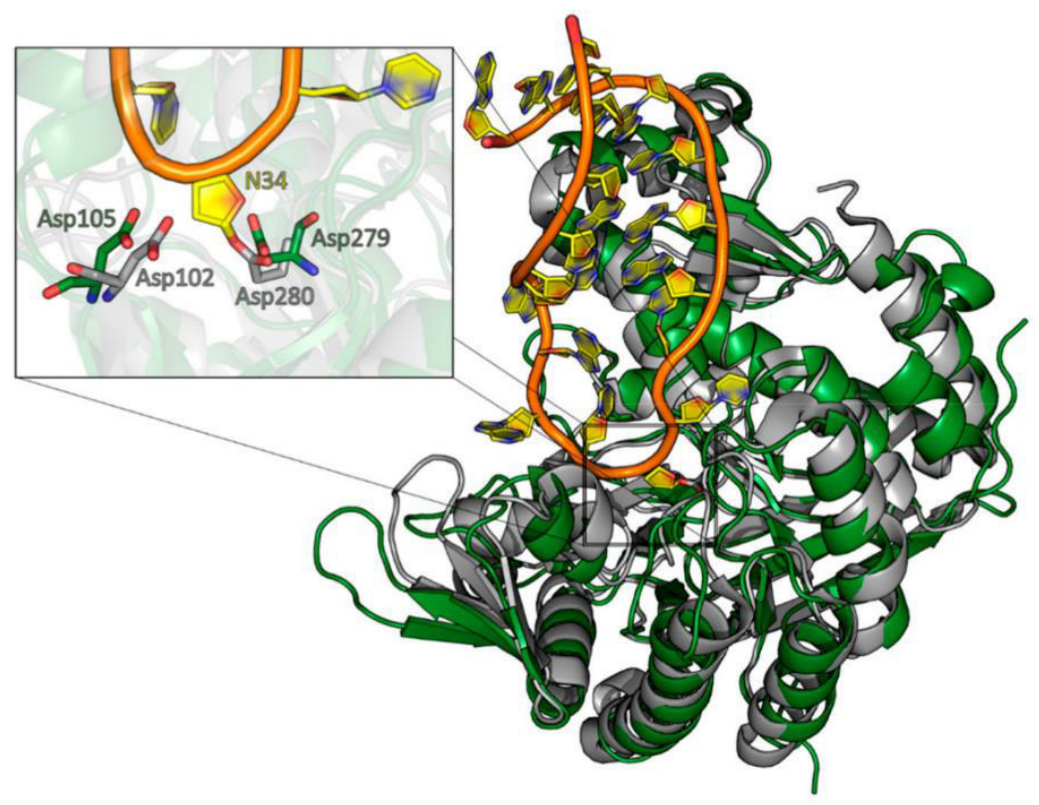

Figure 4. Alignment of the QTRT1 crystal structure with the Z. mobilis TGT RNA complex. The crystal structure of QTRT1 (green) was aligned to the protein part of the Z. mobilis TGT structure in complex with a tRNA anticodon stem loop (PDB-ID: 1Q2R). The complex structure was stalled by addition of 9-de-azaguanine thus exhibiting the deglycosylated state of the guanine 34 . The zoomed view into the active site (left) shows the covalent link of the N34 ribose to the catalytic Asp280 residue and the close by Asp102, which is suggested to perform the nucleophilic attack on preQ1 in the bacterial enzyme. The overlay with QTRT1 finds the proposed catalytic residues D279 and D105 at the respective sites.

While we do observe the structure of QTRT1 to be closely related to the structure of the bacTGT, its atomic model exhibits substantial differences to the previously described structure of the noncatalytic QTRT2 subunit. Previously reported results indicate that this subunit does not exhibit transglycosylation activity due to mutagenesis of active site residues [31]. In particular, we find the QTRT1 Asp279 residue to be replaced by a glutamate in QTRT2, that chemically harbors the potential to carry out a nucleophilic attack, however a potential reaction intermediate could not be released, as for this step the necessary Asp105 is replaced by a cysteine in QTRT2. Furthermore, we find the queuine binding site to be degenerated in QTRT2. While in QTRT1 queuine is coordinated by stacking between Phe109 and Met259 we do observe dislocation of the respective QTRT2 Tyr107 residue and a mutation of the methionine to cysteine (Cys252). As a result, high affinity binding of queuine to the active site of QTRT2 is unlikely. Compared to QTRT2, QTRT1 harbors all catalytic residues and is capable of binding the modified base. However previously reported biochemical assays reported that QTRT1 alone does not facilitate the transglycosylation reaction. From our perspective the heterodimerization with QTRT2 might provide an additional binding scaffold for the tRNA substrate that could be essential for TGT activity, however this needs to be proven experimentally.

Furthermore, we asked why we do observe only QTRT1 in our crystals, although the crystallization drop contained the purified and catalytically active heterodimer. Prior studies of the mouse TGT quaternary structure by Behrens et al., employing mass spectrometry under nondenaturing conditions, evaluated the main fraction of an equimolar mixture of QTRT1 and QTRT2 to form the heterodimeric arrangement of the two subunits. However, the homodimeric subunit arrangements of both QTRT1 and QTRT2 have been observed previously. Interestingly, the vast majority of the QTRT2 in absence of the catalytic subunit formed homodimers. While the biological consequence of this observation remains elusive, the authors were also able to observe a homodimeric arrangement of this subunit in the crystal structure. The same investigation of the catalytic QTRT1 subunit revealed 
the ability of the catalytic subunit to form stable homodimers, however to a much less extend with a fraction of only $20 \%$ of proteins being self-associated in solution. Analysis of the QTRT1 crystallization condition revealed the presence of highly chaotropic thiocyanate, which might promote destabilization of the TGT heterodimer in the crystallization droplet. Furthermore, we do observe a PGA molecule at the interface of the asymmetric units promoting contact of the B-chain with a symmetry mate through stacking between symmetry equivalent Arg184 residues. The interface is further extended by a bromide ion, which is also a component of the crystallization condition. We further evaluated the quaternary structure of both proteins in the asymmetric unit and found the interaction not to be promoted by presence of components of the crystallization condition. However, although the active site is exposed to solvent, binding of a bulky substrate tRNA to one or the other catalytic site is most likely sterically hindered by presence of other molecules in the crystal lattice. We calculated the interaction interface to be of $719.9 \AA^{2}$ with the free dimerization enthalpy of $\Delta \mathrm{G}=-9.8 \mathrm{kcal} / \mathrm{mol}$ which does not suggest that this dimer is stable in solution [48]. Investigation of potential biological assemblies that include one component of the asymmetric unit and a contacting symmetry mate also did not find a stable dimer nor an arrangement that exhibited similar coordination of both subunits comparably to the one observed for the Z. mobilis homodimer or the proposed QTRT2 biological assembly (Figure 5).
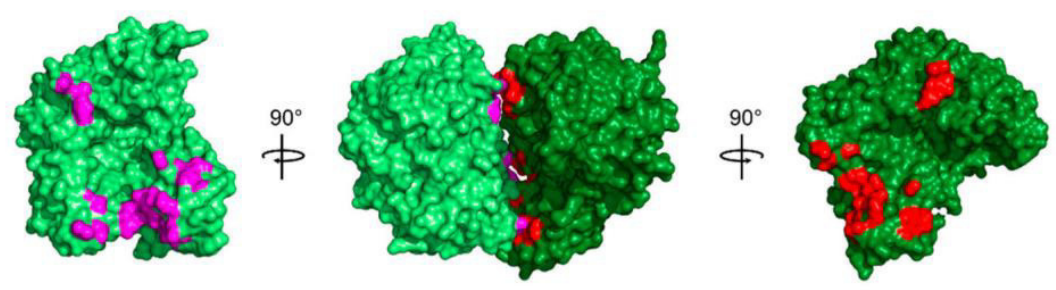

(a)
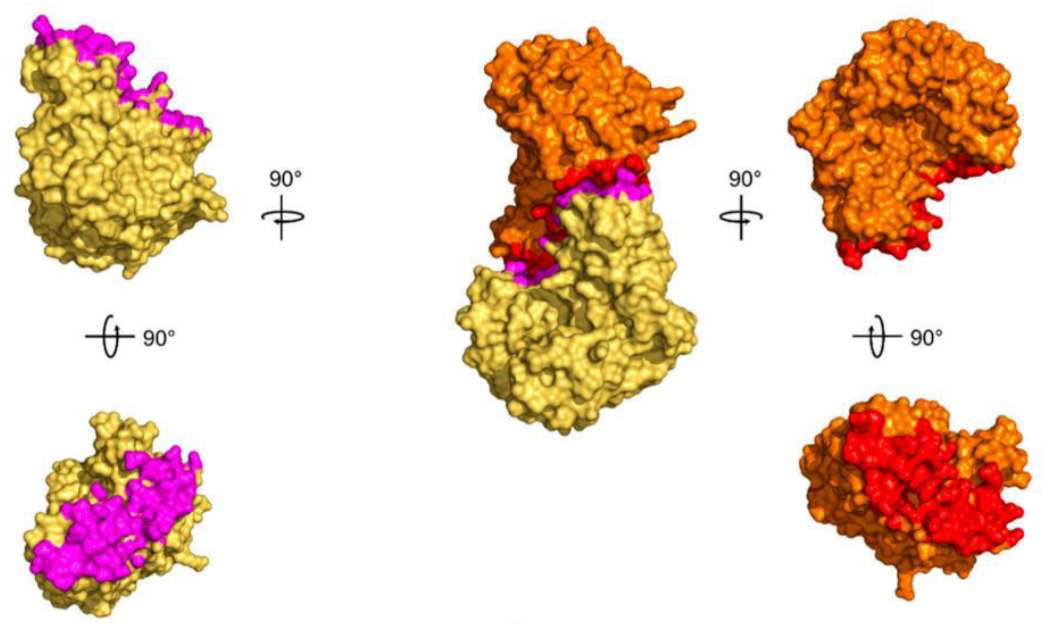

(b)

Figure 5. Comparison of the approximately $720 \AA^{2}$ interface area observed for human QTRT1 with the interface area of the bacterial TGT homodimer. (a) Both QTRT1 molecules A and B are shown in dark green and pale green, respectively. Residues within $5 \AA$ distance of the other molecule are shown in red for molecule A and in magenta for molecule B. (b) The interface area between the Z. mobilis TGT (PDB-ID: 1PUD) homodimer subunits, that are depicted in orange and pale orange, extends to approximately $1600 \AA^{2}$. The orange subunit was aligned to QTRT1 molecule A (dark green) to outline the different interaction site compared to QTRT1. Residues within $5 \AA$ of the respective other subunit are again presented in red and magenta. All structures in (a) and (b) are depicted as surface representations. 
Therefore, our crystal structure supports previous observations that QTRT1 is mainly monomeric in solution, and the dimer architecture we observe in the asymmetric unit is likely caused by crystal packing. The structures of QTRT1 and QTRT2 both support previous observations that a fraction of the eukaryotic TGT heterodimer is dissociated in solution however further biochemical studies are necessary to identify its biological significance.

The eukaryotic TGT has been proven to incorporate the fully modified queuine base, however, the introduction of queuine into the tRNA might not represent the final step for all queuine molecules after incorporation. Queuine has been observed to undergo further modification by $O$-mannosylation at the C4 hydroxy group of the cyclopentene diol moiety in humans and rats $[49,50]$. The question arises if the free queuine base is glycosylated or this hypermodification is established after introduction into the tRNA by TGT. While in humans only the O-linkage of mannose was observed, $\mathrm{O}$-mannose or $O$-galactose was found to occur on queuine in samples derived from rat liver [50]. Biochemical investigation of the isolated, endogenous glycosyltransferase from rat liver found the enzyme to be specific for queuine in the context of tRNA ${ }^{\text {Asp }}$ as no mannosylation was observed for Q34tRNA ${ }^{\text {Asn }}$ and Q34tRNA ${ }^{\text {His }}$ while tRNA ${ }^{\text {Tyr }}$ was found to harbor galactose modification exclusively [51]. Therefore, the target seems to be defined not only by queuine chemical structure but also by tRNA structural properties. In our crystal structure of the queuine QTRT1 complex, we do observe the queuine cyclopentene $\mathrm{C} 4 \mathrm{OH}$ group to be exposed to the solvent. Presence of an additional sugar at this position would be possible and likely not interfere with coordination of the queuine base by the identified residues. Therefore, our QTRT1 crystal structure renders binding of a glycosylated queuine base into the active site possible in absence of a tRNA substrate. However, whether this would also be the case with a bound tRNA molecule still needs to be structurally investigated.

\section{Conclusions}

The herein reported structure of the human QTRT1 provides the first structural insights into the structural arrangement of a eukaryotic TGT active subunit and its active site. While previous structural investigations had to rely on homology modeling and engineering of the bacterial TGT, the herein reported crystal structures provide, for the first time, direct structural data about the location of catalytic residues in the active site and how the queuine substrate is bound. Furthermore, our results provide strong evidence for the previously deducted conservation of the TGT catalytic mechanism from eubacteria to eukaryotes.

Author Contributions: S.J. and R.F. designed the experiments. S.J. performed cloning and purification of proteins. Crystallization and sample preparation was done by S.J. S.J. also performed initial molecular replacement. PN did the MR-SAD experiment and respective data interpretation. Building of the final structures was performed by S.J. The structures were interpreted by S.J., P.N. and R.F. S.J., P.N. and R.F. prepared the manuscript.

Funding: This work was supported with funding by the DFG (priority program SPP1784, INST 186/814-1, and INST 186/1117-1) to R.F. S.J. was partly supported by the Göttingen Graduate School for Neurosciences, Biophysics, and molecular biosciences (DFG grant GSC 226/2).

Acknowledgments: The synchrotron MX data was collected at beamline P14 operated by EMBL Hamburg at the PETRA III storage ring (DESY, Hamburg, Germany). We would like to thank Saravanan Panneerselvam for the assistance in using the beamline. Furthermore, we are most grateful for the queuine base which was provided by Matthias Krull and Ulf Diederichsen (University of Göttingen) and acknowledge the support of Nora Eulig during protein crystallization.

Conflicts of Interest: The authors declare no conflicts of interest. 


\section{Appendix A}

\begin{tabular}{|c|c|c|}
\hline Data Collection & QTRT1 & QTRT1·Queuine \\
\hline PDB-ID & $6 \mathrm{H} 42$ & $6 \mathrm{H} 45$ \\
\hline X-ray source & Synchrotron (PETRA III, P14) & Synchrotron (PETRA III, P14) \\
\hline Detector & Dectris Eiger & Dectris Eiger \\
\hline Wavelength $(\AA)$ & 0.97626 & 0.97626 \\
\hline Space group & $\mathrm{C} 2$ & $\mathrm{C} 2$ \\
\hline \multicolumn{3}{|l|}{ Cell dimensions } \\
\hline$a, b, c(\AA)$ & $127.70,157.82,47.77$ & $127.50,158.86,47.62$ \\
\hline$\alpha, \beta, \gamma\left({ }^{\circ}\right)$ & $90.00,108.46,90.00$ & $90.00,108.20,90.00$ \\
\hline Resolution $(\AA)$ & $48.25-2.45(2.55-2.45)$ & $48.16-2.40(2.50-2.40)$ \\
\hline $\mathrm{R}_{\text {meas }}(\%)$ & $10.6(111.2)$ & $5.8(59.0)$ \\
\hline $\mathrm{I} / \sigma(\mathrm{I})$ & $10.3(1.7)$ & $17.3(2.3)$ \\
\hline $\mathrm{CC}_{1 / 2}$ & $99.6(59.9)$ & $99.9(84.7)$ \\
\hline Completeness (\%) & $99.4(99.5)$ & $93.1(67.6)$ \\
\hline Redundancy & $4.5(4.5)$ & $3.3(2.7)$ \\
\hline \multicolumn{3}{|l|}{ Refinement } \\
\hline Resolution $(\AA)$ & $48.25-2.45$ & $48.16-2.40$ \\
\hline No. of reflections & 32849 & 32702 \\
\hline $\mathrm{R}_{\text {work }}(\%)$ & 20.24 & 20.13 \\
\hline$R_{\text {free }}(\%)$ & 23.95 & 23.96 \\
\hline \multicolumn{3}{|l|}{ No. of atoms } \\
\hline Protein & 6033 & 6010 \\
\hline Water & 99 & 103 \\
\hline Ligand & - & 40 \\
\hline Ions & 15 & 13 \\
\hline \multicolumn{3}{|l|}{ B-factors $\left(\AA^{2}\right)$} \\
\hline Protein & 67.3 & 58.0 \\
\hline Ligand & - & 73.7 \\
\hline Water and other small molecules & 61.7 & 71.7 \\
\hline \multicolumn{3}{|l|}{ r.m.s. deviations } \\
\hline Bond lengths $(\AA)$ & 0.0037 & 0.0074 \\
\hline Bond angles $\left({ }^{\circ}\right)$ & 0.643 & 0.814 \\
\hline \multicolumn{3}{|l|}{ MolProbity analysis } \\
\hline Ramachandran favored (\%) & 96.5 & 96.6 \\
\hline Ramachandran outliers (\%) & 0.0 & 0.0 \\
\hline Rotamer outliers (\%) & 1.37 & 1.99 \\
\hline Clash score & 4.96 & 5.35 \\
\hline
\end{tabular}




\section{Appendix B}

Sequence alignment of a bacterial TGT and eukaryotic TGT subunits. Sequences of the Z.mobilis TGT and mouse QTRT2 have been aligned to the sequence of human QTRT1 (UniProtKB entries P28720 B8ZXI1 and Q9BXR0) using T-Coffee.

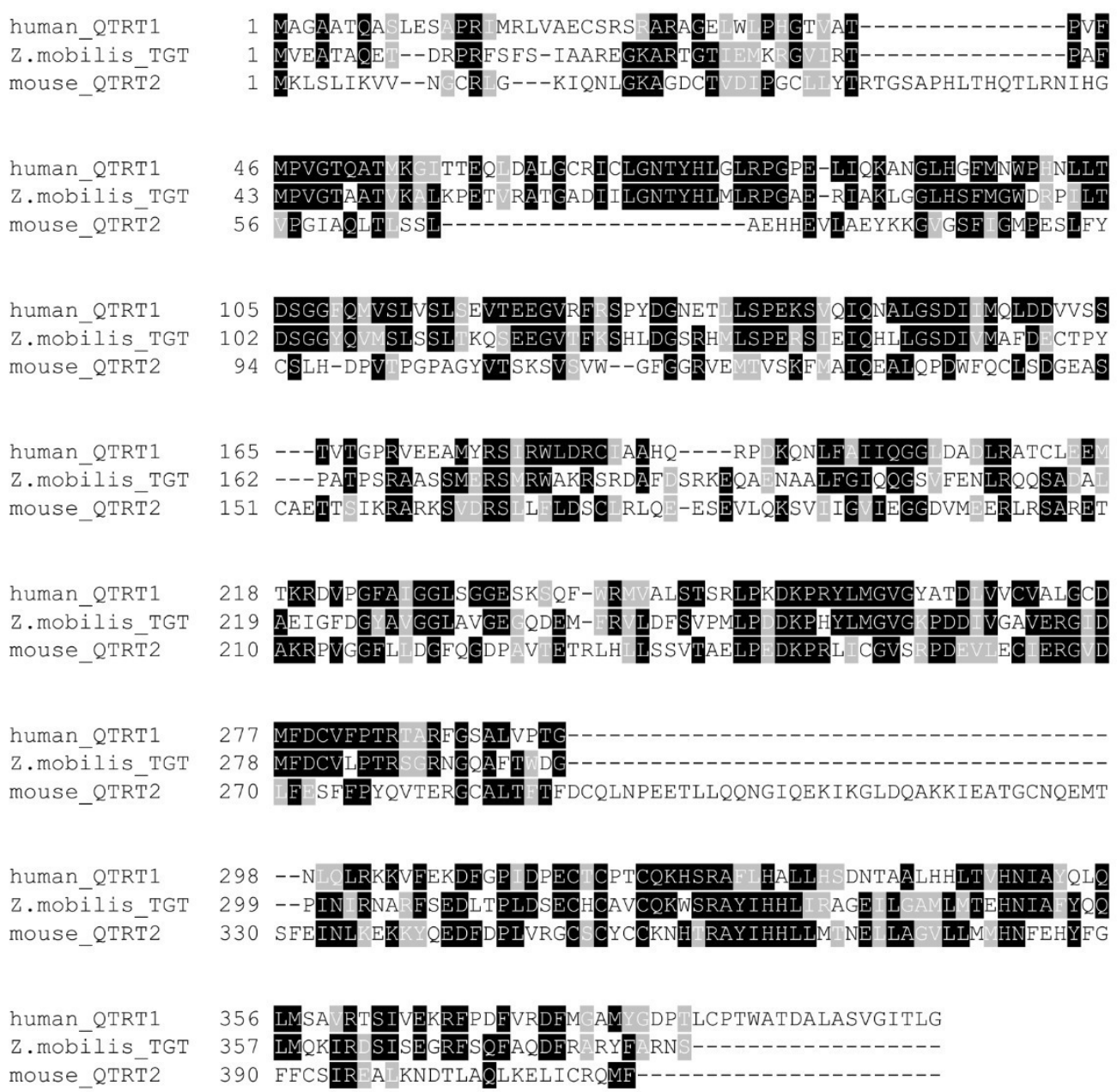

\section{References}

1. Boccaletto, P.; Machnicka, M.A.; Purta, E.; Piątkowski, P.; Bagiński, B.; Wirecki, T.K.; de Crécy-Lagard, V.; Ross, R.; Limbach, P.A.; Kotter, A.; et al. MODOMICS: A database of RNA modification pathways. 2017 update. Nucleic Acids Res. 2018, 46, D303-D307. [CrossRef] [PubMed]

2. Sokołowski, M.; Klassen, R.; Bruch, A.; Schaffrath, R.; Glatt, S. Cooperativity between different tRNA modifications and their modification pathways. Biochim. Biophys. Acta BBA Gene Regul. Mech. 2018, 1861, 409-418. [CrossRef] [PubMed]

3. Nachtergaele, S.; He, C. The emerging biology of RNA post-transcriptional modifications. RNA Biol. 2017, 14, 156-163. [CrossRef] [PubMed]

4. Lorenz, C.; Lünse, C.E.; Mörl, M. tRNA Modifications: Impact on structure and thermal adaptation. Biomolecules 2017, 7, 35. [CrossRef] [PubMed]

5. Tuorto, F.; Herbst, F.; Alerasool, N.; Bender, S.; Popp, O.; Federico, G.; Reitter, S.; Liebers, R.; Stoecklin, G.; Gröne, H.-J.; et al. The tRNA methyltransferase Dnmt2 is required for accurate polypeptide synthesis during haematopoiesis. EMBO J. 2015, 34, 2350-2362. [CrossRef] [PubMed]

6. Harada, F.; Nishimura, S. Possible anticodon sequences of tRNA His, tRNA Asm, and tRNA Asp from Escherichia coli $\mathrm{B}$. Universal presence of nucleoside $\mathrm{Q}$ in the first postion of the anticondons of these transfer ribonucleic acids. Biochemistry 1972, 11, 301-308. [CrossRef] [PubMed] 
7. Phillips, G.; Yacoubi, B.E.; Lyons, B.; Alvarez, S.; Iwata-Reuyl, D.; de Crécy-Lagard, V. Biosynthesis of 7-Deazaguanosine-Modified tRNA Nucleosides: A New Role for GTP Cyclohydrolase I. J. Bacteriol. 2008, 190, 7876-7884. [CrossRef] [PubMed]

8. McCarty, R.M.; Somogyi, Á.; Bandarian, V. Escherichia coli QueD Is a 6-Carboxy-5,6,7,8-Tetrahydropterin Synthase. Biochemistry 2009, 48, 2301-2303. [CrossRef] [PubMed]

9. McCarty, R.M.; Somogyi, Á.; Lin, G.; Jacobsen, N.E.; Bandarian, V. The deazapurine biosynthetic pathway revealed: In vitro enzymatic synthesis of PreQ0 from Guanosine 5'-Triphosphate in four steps. Biochemistry 2009, 48, 3847-3852. [CrossRef] [PubMed]

10. Dowling, D.P.; Bruender, N.A.; Young, A.P.; McCarty, R.M.; Bandarian, V.; Drennan, C.L. Radical SAM enzyme QueE defines a new minimal core fold and metal-dependent mechanism. Nat. Chem. Biol. 2014, 10, 106-112. [CrossRef] [PubMed]

11. Lanen, S.G.V.; Reader, J.S.; Swairjo, M.A.; de Crécy-Lagard, V.; Lee, B.; Iwata-Reuyl, D. From cyclohydrolase to oxidoreductase: Discovery of nitrile reductase activity in a common fold. Proc. Natl. Acad. Sci. USA 2005, 102, 4264-4269. [CrossRef] [PubMed]

12. Lee, B.W.K.; Van Lanen, S.G.; Iwata-Reuyl, D. Mechanistic Studies of Bacillus subtilis QueF, the nitrile oxidoreductase involved in queuosine biosynthesis. Biochemistry 2007, 46, 12844-12854. [CrossRef] [PubMed]

13. Chikwana, V.M.; Stec, B.; Lee, B.W.K.; de Crécy-Lagard, V.; Iwata-Reuyl, D.; Swairjo, M.A. Structural basis of biological nitrile reduction. J. Biol. Chem. 2012, 287, 30560-30570. [CrossRef] [PubMed]

14. Goodenough-Lashua, D.M.; Garcia, G.A. tRNA-Guanine Transglycosylase from E. coli: A Ping-Pong kinetic mechanism is consistent with nucleophilic catalysis. Bioorg. Chem. 2003, 31, 331-344. [CrossRef]

15. Xie, W.; Liu, X.; Huang, R.H. Chemical trapping and crystal structure of a catalytic tRNA guanine transglycosylase covalent intermediate. Nat. Struct. Mol. Biol. 2003, 10, 781-788. [CrossRef] [PubMed]

16. Romier, C.; Reuter, K.; Suck, D.; Ficner, R. Crystal structure of tRNA-guanine transglycosylase: RNA modification by base exchange. EMBO J. 1996, 15, 2850-2857. [PubMed]

17. Romier, C.; Meyer, J.E.; Suck, D. Slight sequence variations of a common fold explain the substrate specificities of tRNA-guanine transglycosylases from the three kingdoms. FEBS Lett. 1997, 416, 93-98. [CrossRef]

18. Ishitani, R.; Nureki, O.; Fukai, S.; Kijimoto, T.; Nameki, N.; Watanabe, M.; Kondo, H.; Sekine, M.; Okada, N.; Nishimura, S.; et al. Crystal structure of archaeosine tRNA-guanine transglycosylase. J. Mol. Biol. 2002, 318, 665-677. [CrossRef]

19. Watanabe, M.; Nameki, N.; Matsuo-Takasaki, M.; Nishimura, S.; Okada, N. tRNA Recognition of tRNA-guanine transglycosylase from a hyperthermophilic archaeon, Pyrococcus horikoshii. J. Biol. Chem. 2001, 276, 2387-2394. [CrossRef] [PubMed]

20. Ishitani, R.; Nureki, O.; Nameki, N.; Okada, N.; Nishimura, S.; Yokoyama, S. Alternative tertiary structure of tRNA for recognition by a posttranscriptional modification enzyme. Cell 2003, 113, 383-394. [CrossRef]

21. Romier, C.; Reuter, K.; Suck, D.; Ficner, R. Mutagenesis and crystallographic studies of Zymomonas mobilis tRNA-guanine transglycosylase reveal aspartate 102 as the active site nucleophile. Biochemistry 1996, 35, 15734-15739. [CrossRef] [PubMed]

22. Garcia, G.A.; Chervin, S.M.; Kittendorf, J.D. Identification of the rate-determining step of tRNA-guanine transglycosylase from Escherichia coli. Biochemistry 2009, 48, 11243-11251. [CrossRef] [PubMed]

23. Durand, J.M.; Okada, N.; Tobe, T.; Watarai, M.; Fukuda, I.; Suzuki, T.; Nakata, N.; Komatsu, K.; Yoshikawa, M.; Sasakawa, C. vacC, a virulence-associated chromosomal locus of Shigella flexneri, is homologous to tgt, a gene encoding tRNA-guanine transglycosylase (Tgt) of Escherichia coli K-12. J. Bacteriol. 1994, 176, 4627-4634. [CrossRef] [PubMed]

24. Durand, J.M.B.; Dagberg, B.; Uhlin, B.E.; Björk, G.R. Transfer RNA modification, temperature and DNA superhelicity have a common target in the regulatory network of the virulence of Shigella flexneri: The expression of the virF gene. Mol. Microbiol. 2000, 35, 924-935. [CrossRef] [PubMed]

25. Hurt, J.K.; Olgen, S.; Garcia, G.A. Site-specific modification of Shigella flexneri virF mRNA by tRNA-guanine transglycosylase in vitro. Nucleic Acids Res. 2007, 35, 4905-4913. [CrossRef] [PubMed]

26. Barandun, L.J.; Immekus, F.; Kohler, P.C.; Ritschel, T.; Heine, A.; Orlando, P.; Klebe, G.; Diederich, F. High-affinity inhibitors of Zymomonas mobilis tRNA-guanine transglycosylase through convergent optimization. Acta Crystallogr. D Biol. Crystallogr. 2013, 69, 1798-1807. [CrossRef] [PubMed] 
27. Immekus, F.; Barandun, L.J.; Betz, M.; Debaene, F.; Petiot, S.; Sanglier-Cianferani, S.; Reuter, K.; Diederich, F.; Klebe, G. Launching spiking ligands into a protein-protein interface: A promising strategy to destabilize and break interface formation in a tRNA modifying enzyme. ACS Chem. Biol. 2013, 8, 1163-1178. [CrossRef] [PubMed]

28. Jeltsch, A.; Ehrenhofer-Murray, A.; Jurkowski, T.P.; Lyko, F.; Reuter, G.; Ankri, S.; Nellen, W.; Schaefer, M.; Helm, M. Mechanism and biological role of Dnmt2 in Nucleic Acid Methylation. RNA Biol. 2017, 14, 1108-1123. [CrossRef] [PubMed]

29. Reyniers, J.P.; Pleasants, J.R.; Wostmann, B.S.; Katze, J.R.; Farkas, W.R. Administration of exogenous queuine is essential for the biosynthesis of the queuosine-containing transfer RNAs in the mouse. J. Biol. Chem. 1981, 256, 11591-11594. [PubMed]

30. Katze, J.R.; Gündüz, U.; Smith, D.L.; Cheng, C.S.; McCloskey, J.A. Evidence that the nucleic acid base queuine is incorporated intact into tRNA by animal cells. Biochemistry 1984, 23, 1171-1176. [CrossRef] [PubMed]

31. Chen, Y.C.; Kelly, V.P.; Stachura, S.V.; Garcia, G.A. Characterization of the human tRNA-guanine transglycosylase: Confirmation of the heterodimeric subunit structure. RNA 2010, 16, 958-968. [CrossRef] [PubMed]

32. Behrens, C.; Biela, I.; Petiot-Bécard, S.; Botzanowski, T.; Cianférani, S.; Sager, C.P.; Klebe, G.; Heine, A.; Reuter, K. Homodimer architecture of QTRT2, the noncatalytic subunit of the eukaryotic tRNA-Guanine Transglycosylase. Biochemistry 2018. [CrossRef] [PubMed]

33. Baranowski, W.; Dirheimer, G.; Jakowicki, J.A.; Keith, G. Deficiency of queuine, a highly modified purine base, in transfer RNAs from primary and metastatic ovarian malignant tumors in women. Cancer Res. 1994, 54, 4468-4471. [PubMed]

34. Huang, B.-S.; Wu, R.-T.; Chien, K.-Y. Relationship of the queuine content of transfer ribonucleic acids to histopathological grading and survival in human lung cancer. Cancer Res. 1992, 52, 4696-4700. [PubMed]

35. Dirheimer, G.; Baranowski, W.; Keith, G. Variations in tRNA modifications, particularly of their queuine content in higher eukaryotes. Its relation to malignancy grading. Biochimie 1995, 77, 99-103. [CrossRef]

36. Rakovich, T.; Boland, C.; Bernstein, I.; Chikwana, V.M.; Iwata-Reuyl, D.; Kelly, V.P. Queuosine deficiency in eukaryotes compromises tyrosine production through increased tetrahydrobiopterin oxidation. J. Biol. Chem. 2011, 286, 19354-19363. [CrossRef] [PubMed]

37. Reisser, T.; Langgut, W.; Kersten, H. The nutrient factor queuine protects HeLa cells from hypoxic stress and improves metabolic adaptation to oxygen availability. Eur. J. Biochem. 1994, 221, 979-986. [CrossRef] [PubMed]

38. Johannsson, S.; Neumann, P.; Wulf, A.; Welp, L.M.; Gerber, H.-D.; Krull, M.; Diederichsen, U.; Urlaub, H.; Ficner, R. Structural insights into the stimulation of $S$. pombe Dnmt2 catalytic efficiency by the tRNA nucleoside queuosine. Sci. Rep. 2018, 8, 8880. [CrossRef] [PubMed]

39. Müller, M.; Hartmann, M.; Schuster, I.; Bender, S.; Thüring, K.L.; Helm, M.; Katze, J.R.; Nellen, W.; Lyko, F.; Ehrenhofer-Murray, A.E. Dynamic modulation of Dnmt2-dependent tRNA methylation by the micronutrient queuine. Nucleic Acids Res. 2015, 43, 10952-10962. [CrossRef] [PubMed]

40. Kabsch, W. XDS. Acta Crystallogr. D Biol. Crystallogr. 2010, 66, 125-132. [CrossRef] [PubMed]

41. McCoy, A.J.; Grosse-Kunstleve, R.W.; Adams, P.D.; Winn, M.D.; Storoni, L.C.; Read, R.J. Phaser crystallographic software. J. Appl. Crystallogr. 2007, 40, 658-674. [CrossRef] [PubMed]

42. Winn, M.D.; Ballard, C.C.; Cowtan, K.D.; Dodson, E.J.; Emsley, P.; Evans, P.R.; Keegan, R.M.; Krissinel, E.B.; Leslie, A.G.W.; McCoy, A.; et al. Overview of the CCP4 suite and current developments. Acta Crystallogr. D Biol. Crystallogr. 2011, 67, 235-242. [CrossRef] [PubMed]

43. Leaver-Fay, A.; Tyka, M.; Lewis, S.M.; Lange, O.F.; Thompson, J.; Jacak, R.; Kaufman, K.; Renfrew, P.D.; Smith, C.A.; Sheffler, W.; et al. ROSETTA3: An object-oriented software suite for the simulation and design of macromolecules. Methods Enzymol. 2011, 487, 545-574. [CrossRef] [PubMed]

44. Adams, P.D.; Afonine, P.V.; Bunkóczi, G.; Chen, V.B.; Davis, I.W.; Echols, N.; Headd, J.J.; Hung, L.-W.; Kapral, G.J.; Grosse-Kunstleve, R.W.; et al. PHENIX: A comprehensive Python-based system for macromolecular structure solution. Acta Crystallogr. D Biol. Crystallogr. 2010, 66, 213-221. [CrossRef] [PubMed]

45. Emsley, P.; Lohkamp, B.; Scott, W.G.; Cowtan, K. Features and development of Coot. Acta Crystallogr. D Biol. Crystallogr. 2010, 66, 486-501. [CrossRef] [PubMed] 
46. Brenk, R.; Stubbs, M.T.; Heine, A.; Reuter, K.; Klebe, G. Flexible adaptations in the structure of the tRNA-modifying enzyme tRNA-Guanine transglycosylase and their implications for substrate selectivity, reaction mechanism and structure-based drug design. ChemBioChem 2003, 4, 1066-1077. [CrossRef] [PubMed]

47. Biela, I.; Tidten-Luksch, N.; Immekus, F.; Glinca, S.; Nguyen, T.X.P.; Gerber, H.-D.; Heine, A.; Klebe, G.; Reuter, K. Investigation of specificity determinants in bacterial tRNA-Guanine transglycosylase reveals queuine, the substrate of its eucaryotic counterpart, as inhibitor. PLoS ONE 2013, 8. [CrossRef] [PubMed]

48. Krissinel, E.; Henrick, K. Inference of macromolecular assemblies from crystalline state. J. Mol. Biol. 2007, 372, 774-797. [CrossRef] [PubMed]

49. Goll, M.G.; Kirpekar, F.; Maggert, K.A.; Yoder, J.A.; Hsieh, C.-L.; Zhang, X.; Golic, K.G.; Jacobsen, S.E.; Bestor, T.H. Methylation of tRNA ${ }^{\text {Asp }}$ by the DNA Methyltransferase Homolog Dnmt2. Science 2006, 311, 395-398. [CrossRef] [PubMed]

50. Kasai, H.; Nakanishi, K.; Macfarlane, R.D.; Torgerson, D.F.; Ohashi, Z.; McCloskey, J.A.; Gross, H.J.; Nishimura, S. The structure of $\mathrm{Q}^{*}$ nucleoside isolated from rabbit liver transfer ribonucleic acid. J. Am. Chem. Soc. 1976, 98, 5044-5046. [CrossRef] [PubMed]

51. Nishimura, S. Structure, biosynthesis, and function of queuosine in transfer RNA. In Progress in Nucleic Acid Research and Molecular Biology; Cohn, W.E., Ed.; Academic Press: Cambridge, MA, USA, 1983; Volume 28, pp. 49-73.

(c) 2018 by the authors. Licensee MDPI, Basel, Switzerland. This article is an open access article distributed under the terms and conditions of the Creative Commons Attribution (CC BY) license (http:/ / creativecommons.org/licenses/by/4.0/). 


\section{CHAPTER 3: INSIGHTS INTO DNMT2 STIMULATION BY QUEUOSINE}

This manuscript was originally published in Scientific Reports.

\section{Structural insights into the stimulation of S.pombe Dnmt2 methyltransferase catalytic efficiency by the tRNA nucleoside queuosine}

Sven Johannsson ${ }^{1}$, Piotr Neumann ${ }^{1}$, Alexander Wulf ${ }^{2}$, Luisa M. Welp ${ }^{2}$, Hans-Dieter Gerber ${ }^{3}$, Matthias Krull ${ }^{4}$, Ulf Diederichsen ${ }^{4}$, Henning Urlaub ${ }^{2,5}$ and Ralf Ficner ${ }^{1 *}$

\section{Author affiliations}

1 Department of Molecular Structural Biology, Institute of Microbiology and Genetics, GZMB, Georg-AugustUniversity Göttingen, 37077 Göttingen, Germany

2 Bioanalytical Mass Spectrometry Research Group, Max Planck Institute for Biophysical Chemistry, Am Faßberg 11, 37077 Göttingen, Germany;

3 Institut für Pharmazeutische Chemie der Philipps-Universität Marburg, Marbacher Weg 6, 35032 Marburg, Germany.

4 Institut für Organische und Biomolekulare Chemie, Georg-August-Universität Göttingen, Tammannstrasse 2, 37077, Göttingen, Germany.

5 Bioanalytics, Department of Clinical Chemistry, University Medical Center Göttingen, Robert-Koch-Straße 40, 37075 Göttingen, Germany

* To whom correspondence should be addressed. Tel: +49 551 3914072; Email: rficner@uni-goettingen.de

Scientific Reports, volume 8, Article number: 8880 (2018)

DOI:10.1038/s41598-018-27118

Received: 22 January 2018; Accepted: 24 May 2018; Published: 11 June 2018 


\section{Author contributions:}

S.J. and R.F. designed the experiments. S.J. performed cloning and purification of proteins and tRNA as well as data acquisition at synchrotron and determined the structure. H.-D.G. synthesized the Q-base. M.K. did the herein described novel synthesis of queuine under supervision by U.D. A.W. and L.M.W. performed and analyzed the cross-link experiments under supervision by H.U.. P.N. conducted docking experiments. Data were interpreted by S.J., P.N. and R.F. The manuscript was prepared by S.J. and R.F. 


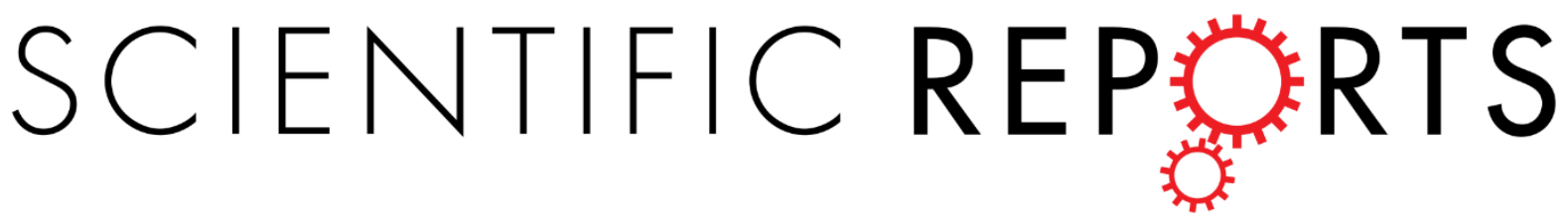

OPEN Structural insights into the stimulation of $S$. pombe Dnmt2 catalytic efficiency by the tRNA nucleoside queuosine

Sven Johannsson ${ }^{1}$, Piotr Neumann ${ }^{1}$, Alexander Wulf ${ }^{2}$, Luisa M. Welp ${ }^{2}$, Hans-Dieter Gerber ${ }^{3}$, Matthias Krull ${ }^{4}$, Ulf Diederichsen ${ }^{4}$, Henning Urlaub ${ }^{2,5}$ \& Ralf Ficner $\mathbb{D}^{1}$

Dnmt2 methylates cytosine at position 38 of tRNA ${ }^{\text {Asp }}$ in a variety of eukaryotic organisms. A correlation between the presence of the hypermodified nucleoside queuosine $(Q)$ at position 34 of tRNA ${ }^{A s p}$ and the Dnmt2 dependent C38 methylation was recently found in vivo for S. pombe and $D$. discoideum. We demonstrate a direct effect of the Q-modification on the methyltransferase catalytic efficiency in vitro, as $\mathrm{V}_{\text {max }} / \mathrm{K}_{0.5}$ of purified $S$. pombe Dnmt 2 shows an increase for in vitro transcribed $t R N A^{A s p}$ containing 034 to $6.27 * 10^{-3} \mathrm{~s}^{-1} \mu \mathrm{M}^{-1}$ compared to $1.51 * 10^{-3} \mathrm{~s}^{-1} \mu \mathrm{M}^{-1}$ for the unmodified substrate. Q34tRNA Asp exhibits an only slightly increased affinity for Dnmt2 in comparison to unmodified G34tRNA. In order to get insight into the structural basis for the Q-dependency, the crystal structure of S. pombe Dnmt2 was determined at $1.7 \AA$ resolution. It closely resembles the known structures of human and $E$. histolytica Dnmt2, and contains the entire active site loop. The interaction with tRNA was analyzed by means of mass-spectrometry using UV cross-linked Dnmt2-tRNA complex. These cross-link data and computational docking of Dnmt2 and tRNA ${ }^{\text {Asp }}$ reveal $Q 34$ positioned adjacent to the S-adenosylmethionine occupying the active site, suggesting that the observed increase of Dnmt2 catalytic efficiency by queuine originates from optimal positioning of the substrate molecules and residues relevant for methyl transfer.

An amazing diversity of chemically modified nucleosides has been identified in various RNAs in all kingdoms of life ${ }^{1,2}$. At least 93 different modifications were found in tRNA molecules. Interestingly, modification of tRNA is not just a static posttranscriptional step in tRNA maturation, but can also be dynamic and might be part of a response to stimuli such as stress ${ }^{3}$. Modifications in the anticodon-loop of tRNAs are known or proposed to affect the codon-anticodon interaction and therefore the fidelity and rate of translation, while modifications in other regions of the tRNA mainly modulate the stability and flexibility of tRNAs ${ }^{4,5}$. However, modifications of tRNAs might have also other important functions. For example, C5-methylation of C38 in the tRNA anticodon-loop by the methyltransferase Dnmt2 was shown to protect tRNAs from cleavage ${ }^{6}$, which in turn has an impact on Dicer2-dependent siRNA pathways ${ }^{7}$. Methylation of C38 also promotes the aminoacylation of tRNA $^{\text {Asp }}$ in vitro $^{8}$, and contributes to translational accuracy?

Recently, the C38 methylation of tRNA ${ }^{\text {Asp }}$ in S. pombe was found to depend on the presence of queuosine $(\mathrm{Q})$, a hypermodified nucleoside at position 34 of $\mathrm{tRNA}^{\text {Asp }}$, $\mathrm{tRNA}^{\mathrm{Asn}}, \mathrm{tRNA}^{\mathrm{Ty}}$, and $\mathrm{tRNA} \mathrm{Ais}^{\mathrm{His}}$. Even though $\mathrm{Q}$ was discovered more than 40 years ago $^{11}$, its common physiological role in eubacteria and eukaryotes is still unclear ${ }^{12}$. Due to its position in the anticodon as wobble base, $\mathrm{Q}$ has been believed to modulate codon-anticodon interaction, however, a clear effect on mRNA translation has yet not been demonstrated. Crystal structures of a ribosome

${ }^{1}$ Department of Molecular Structural Biology, Institute of Microbiology and Genetics, GZMB, Georg-AugustUniversity Göttingen, 37077, Göttingen, Germany. ${ }^{2}$ Bioanalytical Mass Spectrometry Research Group, Max Planck Institute for Biophysical Chemistry, Am Faßberg 11, 37077, Göttingen, Germany. ${ }^{3}$ Institut für Pharmazeutische Chemie der Philipps-Universität Marburg, Marbacher Weg 6, 35032, Marburg, Germany. ${ }^{4}$ Institut für Organische und Biomolekulare Chemie, Georg-August-Universität Göttingen, Tammannstrasse 2, 37077, Göttingen, Germany. ${ }^{5}$ Bioanalytics, Department of Clinical Chemistry, University Medical Center Göttingen, Robert-Koch-Straße 40, 37075 , Göttingen, Germany. Correspondence and requests for materials should be addressed to R.F. (email: rficner@ uni-goettingen.de) 


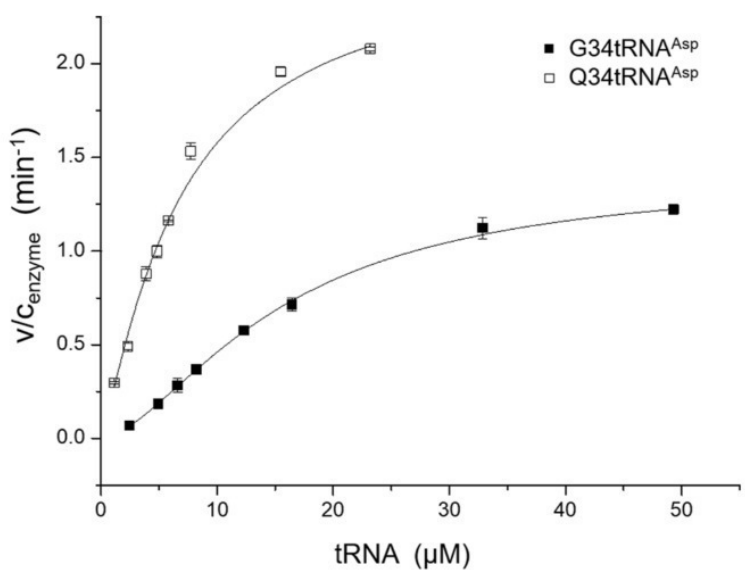

Figure 1. Methyltransferase activity assay with spDnmt2 using unmodified tRNA ${ }^{\text {Asp }}\left(G_{3} 34 \mathrm{tRNA}^{\text {Asp }}\right)$ and queuine harboring tRNA ${ }^{A s p}\left(\mathrm{Q} 34 \mathrm{TRNA}^{\mathrm{Asp}}\right)$ as substrates. Activity is plotted as substrate conversion per enzyme concentration in $\mathrm{min}^{-1}$. Measurements were performed as independent triplicates with increasing substrate concentrations. Errors are presented as standard deviation. Data points were fitted with the Hill equation employing a free Hill coefficient. $V_{\max }$ increased from $1.41 \pm 0.01 \mathrm{~min}^{-1}$ for G34tRNA Asp to $2.59 \pm 0.11 \mathrm{~min}^{-1}$ for Q34tRNA ${ }^{\text {Asp }}$. $\mathrm{K}_{0.5}$ shifted from $15.59 \pm 0.29 \mu \mathrm{M}$ to $6.88 \pm 0.58 \mu \mathrm{M}$ respectively.

with a C-A mismatch of Q34-tRNA ${ }^{\mathrm{Tyr}}$ bound to a His codon revealed a displacement of the cytosine from the codon-anticodon helix and distortion of the latter, hinting to a role of the Q-modification in translational accuracy $^{13}$. While prokaryotes are able to synthesize $\mathrm{Q}$ de novo, it is a nutrient for eukaryotes. Importantly, the enzyme tRNA-guanine-transglycosylase (TGT) replaces the guanine at position 34 of the tRNA by a base exchange mechanism $^{14}$. In case of prokaryotes the $\mathrm{G} 34$ is replaced by the queuine precursor preQ1, and in eukaryotes by queuine. In contrast to bacterial TGT, which is a homo-dimer, human TGT is a functional hetero-dimer consisting of a catalytically active subunit (QTRT1), and a homologous, but catalytically inactive subunit (QTRTD1) ${ }^{15}$.

The Q-dependency of tRNA ${ }^{\text {Asp }}$ C38 methylation was found in vivo, and the effect holds true for purified DNMT2 and tRNA extracted from S. pombe grown under queuine-deficient conditions ${ }^{10}$. However, this did not exclude the possibility that other tRNA modifications might be involved in the stimulation of DNMT2 as well, as the tRNA used was lacking $Q$, but was otherwise fully modified.

Here we demonstrate that Q34 of tRNA ${ }^{\text {Asp }}$ solely is sufficient to increase Dnmt2 catalytic efficiency. We present the crystal structure of S. pombe Dnmt2 and propose a model of the Dnmt2-tRNA complex, based on in silico docking and mass spectrometric analysis of cross-links obtained from the Dnmt2-tRNA complex, showing that Q34 is part of the active site.

\section{Results}

Methlytransferase efficiency is stimulated by 034 in tRNA Asp. Previous data from Müller et al. with purified recombinant Dnmt2 and tRNA extracted from a Dnmt2 deletion mutant of $S$. pombe showed decreased activity of Dnmt2 with tRNA prepared from S. pombe grown under queuine deficient conditions. To rule out an involvement of other modifications present on the used tRNA ${ }^{\text {Asp }}$ we established a system completely outside of $S$. pombe. Dnmt2 of S. pombe (spDnmt2) was recombinantly expressed and purified while tRNA was generated by in vitro transcription. As expected spDnmt 2 was active on this unmodified substrate. To quantify the effect of $Q$ modification in context of tRNA ${ }^{\text {Asp }}$ queuine was enzymatically introduced into tRNA ${ }^{\text {Asp }}\left(\mathrm{Q} 34 \mathrm{tRNA}^{\text {Asp }}\right)$ using the recombinant human tRNA guanine transglycosylase (TGT). Efficiency of spDnmt2 on Q34tRNA Asp was stimulated compared to unmodified G34tRNA Asp (Fig. 1). We observed an increased catalytic efficiency $\left(\mathrm{k}_{\text {cat }} / \mathrm{K}_{0.5}\right)$ from $1.51 * 10^{-3} \mathrm{~s}^{-1} \mu \mathrm{M}^{-1}$ up to $6.27 * 10^{-3} \mathrm{~s}^{-1} \mu \mathrm{M}^{-1}$ for tRNA ${ }^{\text {Asp }}$ when $\mathrm{G} 34$ is exchanged by Q34. $\mathrm{K}_{0.5}$ shifted from $15.60 \pm 0.29 \mu \mathrm{M}$ for G34tRNA ${ }^{\text {Asp }}$ down to $6.88 \pm 0.58 \mu \mathrm{M}$ for Q34tRNA ${ }^{\text {Asp. }}$. These results suggest the increased efficiency to be directly triggered by Q34 modification. We also observed that fit with the Michealis-Menten equation only poorly described the observed data (Supplementary Fig. S6). It was best described by employing the Hill equation as a free fit (Fig. 1). We observed a Hill coefficient of $n=1.63 \pm 0.02$ for the unmodified and $\mathrm{n}=1.19 \pm 0.07$ for the $\mathrm{Q} 34$ modified tRNA substrate.

Affinity of tRNA ${ }^{\text {Asp }}$ to Dnmt2. We further ought to understand how Q34 alters Dnmt2 catalytic efficiency on tRNA ${ }^{\text {Asp }}$. Quantitative analysis of binding with fluorescently labelled tRNAs revealed a change in affinity by Q34 modification (Fig. 2). Presence of queuine in the tRNA resulted in a decrease in $K_{d}$ from $0.96 \pm 0.05 \mu \mathrm{M}$ to $0.67 \pm 0.003 \mu \mathrm{M}$, which, however, may not fully explain the positive effect of Q34 on Dnmt2 efficiency observed in methyltransferase activity assay.

Structural insights into S. pombe Dnmt2. For further insights into the activation of Dnmt2 by Q we solved the spDnmt2 crystal structure in complex with S-adenosyl-homocysteine (SAH) at $1.7 \AA$ resolution by molecular replacement (Fig. 3a). This structure comprises all amino acids from position 5 to the $\mathrm{C}$-terminal end, 


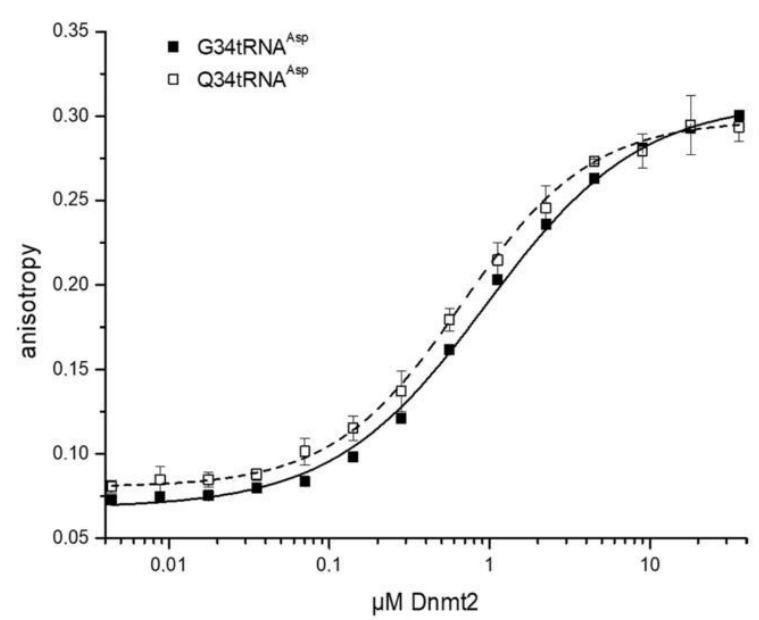

Figure 2. Quantitative analysis of Dnmt2 tRNA complex formation. Binding of the indicated fluorescein labelled G34tRNA Asp and Q34tRNA ${ }^{\text {Asp }}$ was analyzed with increasing spDnmt2 concentration. Complex formation was observed with fluorescence polarization.
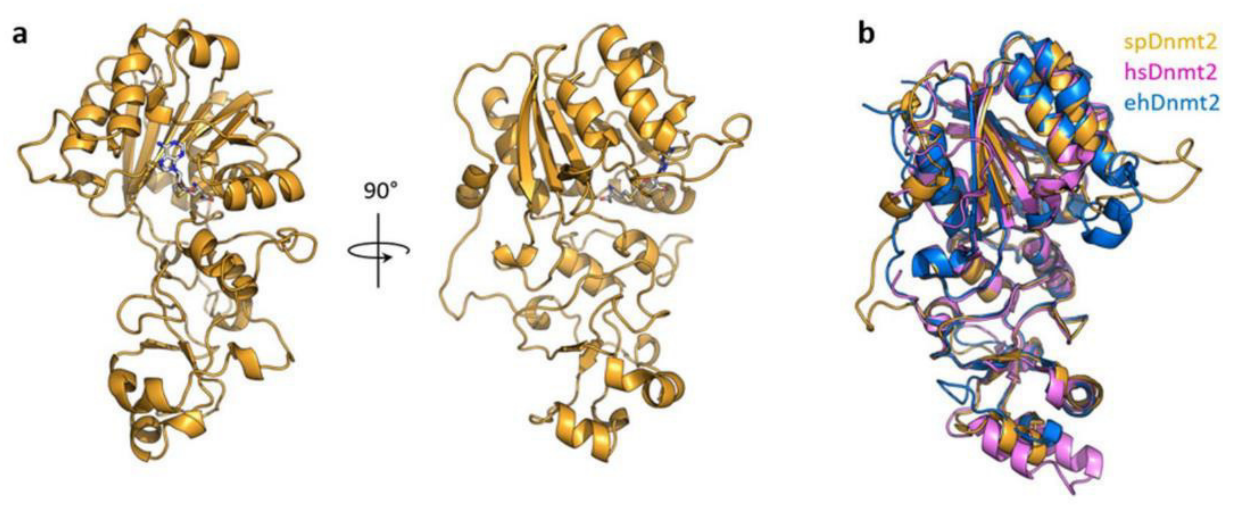

Figure 3. (a) Cartoon representation of S. pombe Dnmt2 crystal structure (yellow) co-crystallized with S-adenosyl-homocysteine depicted as sticks (grey). The crystal structure was refined at $1.7 \AA$ A. (b) Superposition of Dnmt2 crystal structures from S. pombe (yellow), H. sapiens (pink) and E. histolytica (blue) shown as cartoons.

including loop regions, and exhibits an overall similar fold with the Dnmt 2 crystal structures from human (hsD$\mathrm{nmt} 2)^{16}$ and the pathogenic amoebae E. histolytica (ehDnmt2) ${ }^{17}$ (Fig. 3b). In the spDnmt2 structure we were able to build a loop of 20 amino acids starting with Cys 80 , which was proved to be important for catalysis in hsDnmt2 and therefore is referred to this loop as the "active site loop". This loop is presumably unstructured in solution as the electron density for the corresponding amino acids was only observed for one of the four Dnmt 2 molecules in the asymmetric unit, and this loop adopted a defined conformation due to formation of crystal contacts with another Dnmt2 molecule in the crystal lattice. A corresponding loop is also resolved in ehDnmt2 structure where it exhibits a different conformation which appears to be confined by crystal contacts ${ }^{17}$. The crystal structure of hsDnmt2 does not exhibit any traceable loop comprising the corresponding amino acids, arguing flexibility of the active site loop in Dnmt2 of all three organisms.

Analysis of spDnmt2 electrostatic surface potentials unveils predominantly positively charged surface around the SAH indicating possible interaction with the tRNA phosphate backbone. However, the structure exposes a strongly negatively charged cavity in close proximity to the sulfur of SAH formed by the catalytic residue Glu121, which is also conserved in hsDnmt 2 and ehDnmt2. This cavity might accommodate the base of cytosine C 38 , which needs to be flipped out in order to be methylated. The required base flipping has been structurally observed for Dnmtl, which uses the same catalytic mechanism ${ }^{18}$.

Analysis of the DNMT2-tRNA ${ }^{\text {Asp }}$ complex with UV induced cross-linking. To elucidate how queuine impacts Dnmt2 catalytic efficiency we aimed at understanding how Dnmt2 binds its tRNA substrate. 
a

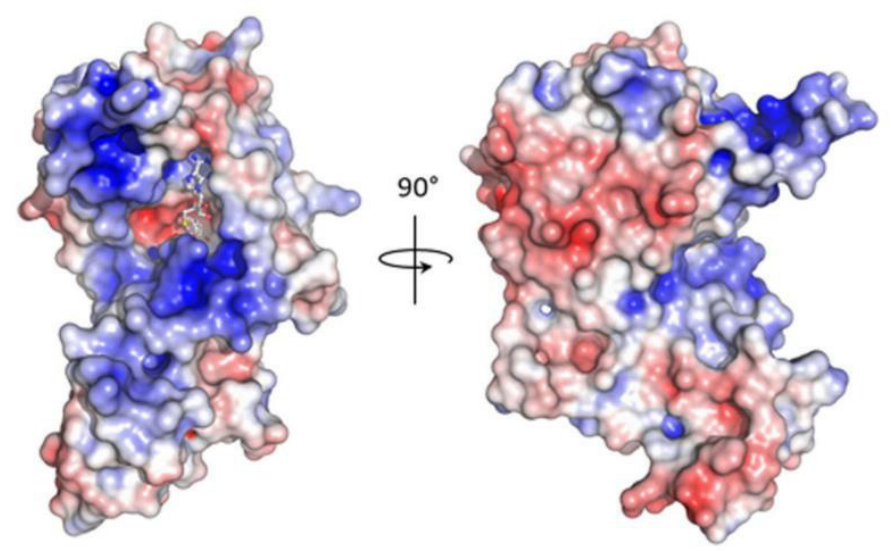

b

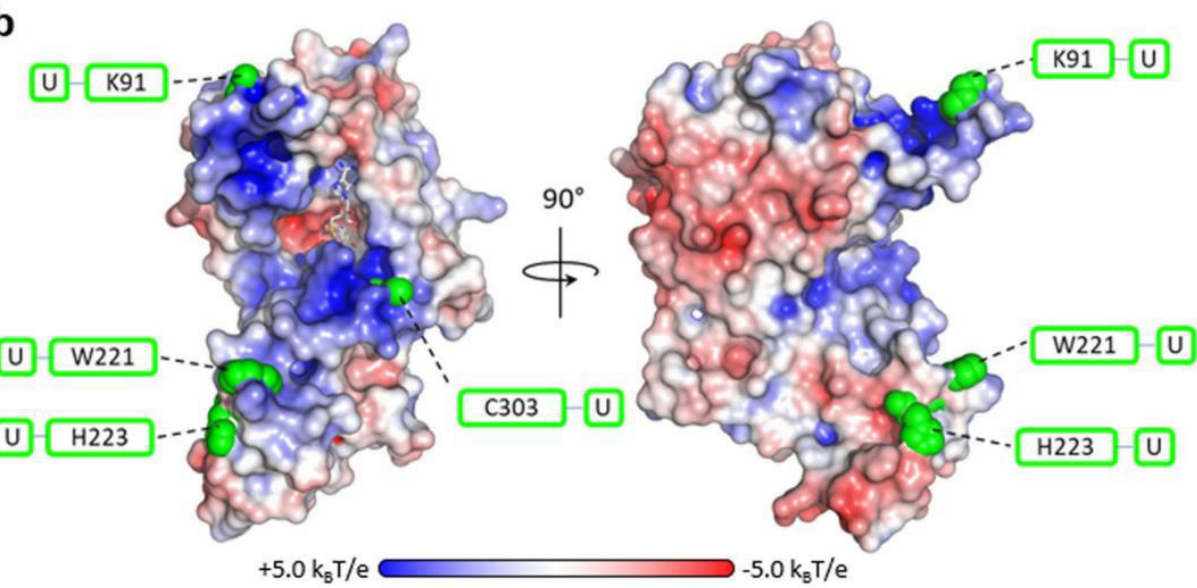

Figure 4. (a) Surface representation of $S$. pombe Dnmt2 electrostatic potentials depicted at a contour level of $\pm 5.0 \mathrm{k}_{\mathrm{B}} \mathrm{T} / \mathrm{e}$. SAH is presented as grey sticks. (b) Structural analysis of protein RNA cross-links. Residues crosslinked to tRNA ${ }^{\text {Asp }}$ are depicted as green spheres. All cross-links were observed as covalent links to uridines.

Therefore, we performed UV light induced cross-linking of the Dnmt2 tRNA ${ }^{\text {Asp }}$ complex and analyzed the cross-linked peptides with mass spectrometry. Four residues cross-linked to the RNA were identified, namely Lys91, Trp221, His223, and Cys303, with each of them cross-linked to a uridine (Fig. 4).

An overlay of spDnmt 2 and hsDnmt 2 reveals that three of these cross-links are positioned in close proximity to residues important for activity in hsDnmt2 (Fig. 5), as mutation of a single of these residues resulted in less than $25 \%$ activity compared to wildtype ${ }^{19}$. The Cys 303 cross-link is in close proximity to a positive patch formed by Arg371, Lys295 and Lys367 in hsDnmt2. Additionally, we find cross-linked residues Trp221 and His 223 to be close to hsDnmt2 Arg275 residue. Lys91 is positioned within the active loop and in close proximity to Arg95, a residue conserved in both S. pombe and human but not resolved in the structure of hsDnmt2. Strikingly, mutation of this residue in human Dnmt2 mostly abolishes activity, highlighting the importance of the flexible loop for Dnmt2 catalytic function.

Dnmt2-tRNA docking places G34 in close proximity to the active site. For a more detailed understanding of how Dnmt2 binds to tRNA and which regions might be involved in Q recognition we performed computational docking of the Dnmt 2 tRNA complex. Cross-linking data was used for model validation but docking was done without applying restrains for the cross-links. For docking we chose the herein described spDnmt2 crystal structure and tRNA structures from the PDB. The best Dnmt2-tRNA model was derived from docking of the crystal structure of yeast tRNA Asp (1VTQ). Afterwards the best docked tRNA ${ }^{\text {Asp }}$ model was manually modified, the G34 was replaced by queuine and the C 38 base was flipped, as the flipped out conformation of this base has shown to be necessary for methylation by Dnmt proteins ${ }^{18}$.

The resulting model of the tRNA Dnmt2 complex (Fig. 6) has the tRNA placed with the anticodon stem in a positively charged groove around the active site. In this orientation tRNA phosphate backbone forms numerous electrostatic interactions with Dnmt2. Interestingly, in this model Q34 is facing the protein surface close to active site with the diol ring close to the SAM binding grove. Furthermore, the cross-linked residues Cys 303 , Trp221 and His223 are adjacent to bases that correspond to uridines in sptRNA ${ }^{\text {Asp }}$ that was used in cross-linking. However cross-linked Lys91 is not positioned close to an uridine in our model, which indicates that the conformation of 

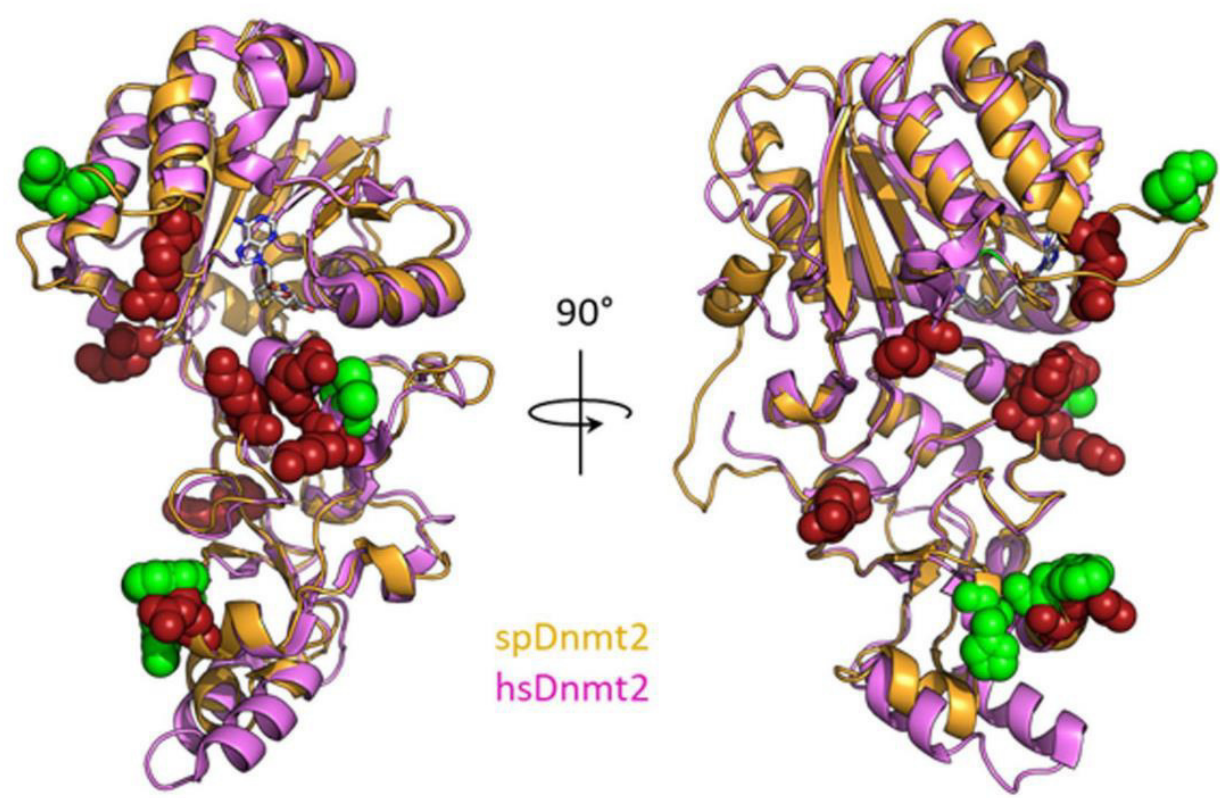

Figure 5. Overlay of spDnmt 2 cross-links to $t R N A^{A s p}$ with lysine and arginine residues known to be important for Dnmt2 catalytic activity in human. spDnmt2 and hsDnmt2 were superimposed and depicted as cartoon. spDnmt2 residues cross-linked to tRNA ${ }^{\text {Asp }}$ are depicted as green spheres, hsDnmt2 residues for which single mutation to alanine resulted in $<25 \%$ activity compared to wildtype are shown as red spheres.

a

b

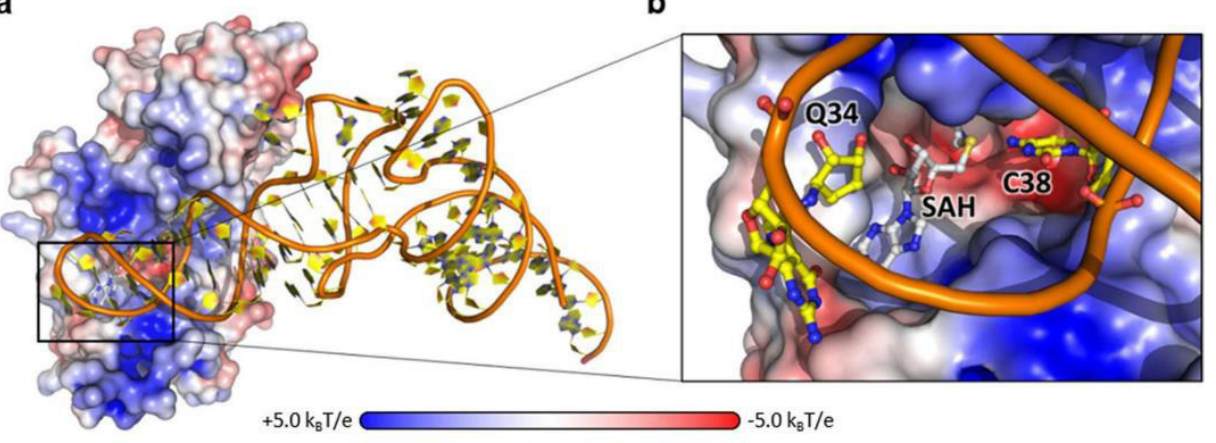

Figure 6. (a) Predicted model of the Dnmt2-tRNA complex. spDnmt2 and yeast tRNA ${ }^{\text {Asp }}$ (1VTQ) were docked computationally using Rosetta. spDnmt2 surface is depicted with surface electrostatics $\left( \pm 5.0 \mathrm{k}_{\mathrm{B}} \mathrm{T} / \mathrm{e}\right)$. tRNA ${ }^{\text {Asp }}$ is shown as cartoon with orange ribose-phosphate backbone and yellow bases. (b) Zoomed view into the active site. Queuine 34, cytosine 38 and S-adenosyl-homocysteine (SAH) are presented as balls and sticks.

the active site loop might not reflect the arrangement of the loop in the Dnmt2-tRNA complex in solution. Given the loop's likely flexibility that we described earlier the loop might exhibit an alternative conformation and be positioned closer to the tRNA when the substrate is bound.

\section{Discussion}

We proved that queuine alone is able to increase spDnmt2 catalytic efficiency when incorporated into position 34 of tRNA ${ }^{\text {Asp }}$ and did quantify this effect with a newly established system completely outside of $S$. pombe. We also observed that the spDnmt2 reaction kinetics do not follow classic Michaelis-Menten kinetics but are better described with a cooperative fit using the Hill equation. From published data and our experience there is no evidence that Dnmt2 is active as a multimer. However, kinetic cooperativity has also been confirmed for monomeric proteins with a single active site. In these cases this observed kinetic cooperativity can be caused by a slow interconversion of two or more conformations of the enzyme upon formation of the enzyme substrate complex, which shifts the equilibrium of these towards the catalytically active conformation ${ }^{20}$. We further could show that 
Q34 increases reaction velocity and lowers $\mathrm{K}_{0.5}$ by almost three fold. At the same time the modification does lower binding affinity by 1.4 fold. These two observations raise the question of how this modification results in the increase of Dnmt 2 catalytic efficiency.

In order to shed light on this question, we solved the reported structure of $S$. pombe Dnmt2. This offers direct insights into the spDnmt2 structural properties and finds its fold to be closely related to Dnmt2 molecules of other organisms. The structure includes a flexible loop adjacent to the active site, which has been found to be crucial for activity of Dnmt2 in human ${ }^{19}$.

Interestingly Pro83 in this loop exhibits a cis-peptide conformation in chains A and B of the crystal structure. This residue is conserved in Dnmt2, but neither in Dnmt1 nor in Dnmt $3^{21}$. Proline has been shown to be the only natural amino acid to form such a stable cis peptide backbone and conversion between cis and trans is hindered by a high energy barrier ${ }^{22,23}$. Rate constants for proline isomerization therefore are minimal and have been shown be as slow as $0.002 \mathrm{~s}^{-124}$. To our knowledge kinetic cooperativity in a monomeric enzyme caused by proline isomerization has not been shown so far, but earlier articles already highlight proline cis-trans isomerization to harbor this potential ${ }^{20}$. Therefore, the isomerization of Pro 83 could serve as a possible explanation for the observed cooperative effect.

Our experimental approach with UV light induced protein RNA cross-linking combined with mass spectrometry provides to our knowledge the first structural data on Dnmt2 tRNA complex formation. Comparison of protein residues involved in these cross-links with a mutational study on human Dnmt $2^{19}$ revealed that the observed cross-links appear in the same or adjacent regions as residues that could be directly linked to hsDnmt 2 activity. Additionally, comparison of the spDnmt 2 crystal structure with structures of Dnmt 2 from other organisms available in the PDB revealed that all structures exhibit an overall similar conformation, arguing in favor of a comprehensively similar substrate binding mechanism by Dnmt 2 across species.

The modeled Dnmt2-tRNA complex reveals Q34 positioned close to the active site but simultaneously too distant from the catalytic residues to directly participate in the methyltransfer reaction. This seems to be likely as Dnmt2 is also active on unmodified G34tRNA ${ }^{\text {Asp }}$.

As revealed by in silico docking experiments, the tRNA's variable loop region and the L-loop, both of which have been shown to be important for methylation by human Dnmt $2^{25}$, are located in vicinity of Dnmt 2 . Additionally, our docking model of the Dnmt2-tRNA complex is in a good agreement with three of the observed four crosslinks. However, the model does not support the fourth (Lys91) cross-link. This residue is harbored inside the active site loop, which in our spDnmt 2 structure is stabilized by crystal contacts. The docking reveals that this conformation does not interfere with the predicted potential binding of the tRNA substrate, however, our result suggests it likely adopts an alternative conformation closer to the RNA when the substrate is bound. This is supported by the mentioned previous mutational analysis of hsDnmt $2^{19}$ which reveals that the active loop harbored and conserved Lys95 is crucial for Dnmt2 activity and as thus is likely to show interaction with the substrate. Furthermore, our Dnmt2-tRNA complex docking model shows a very similar arrangement of nucleotides close to the active site compared with the crystal structure of the Dnmt1-DNA complex which uses the same catalytic mechanism ${ }^{18}$

Finally, the question arises whether the described effect by queuine is restricted to $S$. pombe. Published data describing the analysis of tRNA ${ }^{\text {Asp }}$ modifications in human revealed C38 methylation only to be present in combination with mannosylated queuine at position $34^{25}$. This finding as well as the observed structural similarities of Dnmt 2 across organisms in addition with the observation that tRNA binding of Dnmt2 in S. pombe and human are likely to be similar does argue that the influence of Q34 or hyper-modified Q34 on Dnmt2 might be a more general mechanism in other organisms.

\section{Materials and Methods}

Expression and Purification. Saccharomyces pombe Dnmt2 (spDnmt2) was expressed from pGEX 6P-3 vector as GST-spDnmt2 fusion protein in Escherichia coli BL21(DE3) cells using autoinduction. Cells were grown at $16^{\circ} \mathrm{C}$ for $50 \mathrm{~h}$ before harvesting and stored at $-80^{\circ} \mathrm{C}$ until further use. Cells were disrupted by microfluidization (M-110S Microfluidizer) with $50 \mathrm{mM}$ Tris/ $\mathrm{HCl} \mathrm{pH} \mathrm{7.5,} 150 \mathrm{mM} \mathrm{NaCl}, 1 \mathrm{M} \mathrm{LiCl}, 2 \mathrm{mM}$ DTT and soluble protein was isolated by ultracentrifugation at $50000 \mathrm{~g}$ for $30 \mathrm{~min}$. GST-spDnmt2 was loaded onto Glutathione Sepharose FastFlow (GE Healthcare) (50 mM Tris/HCl pH7.5, $150 \mathrm{mM} \mathrm{NaCl}, 2 \mathrm{mM}$ DTT) and eluted using $30 \mathrm{mM}$ reduced glutathione. GST-tag was cleaved upon addition of PreScission Protease (1:80 w/w, GE Healthcare). GST was removed by heparin-sepharose (GE Healthcare) purification ( $50 \mathrm{mM}$ Tris/ $\mathrm{HCl}$ pH7.5, $100 \mathrm{mM}-2 \mathrm{M} \mathrm{NaCl}, 2 \mathrm{mM}$ DTT). spDnmt2 was further purified by Superdex S75 size exclusion chromatography $(50 \mathrm{mM}$ Tris/ $\mathrm{HCl}, 150 \mathrm{mM}$ $\mathrm{NaCl}, 2 \mathrm{mM}$ DTT). Purified spDnmt 2 was concentrated to $3.5 \mathrm{mg} / \mathrm{mL}$ and stored at $-80^{\circ} \mathrm{C}$ until further use.

Active human tRNA guanine transglycosylase (hTGT) is a heterodimer consisting of QTRT1 and QTRTD1 ${ }^{15}$. QTRT1 and QTRTD1 were co-expressed from pCDF-Duett vector with N-terminally 6xHis tagged QTRT1 (his-hTGT) in Escherichia coli BL21(DE3)-STAR cells using autoinduction. Medium was supplemented with additional $100 \mu \mathrm{M} \mathrm{ZnCl}_{2}$. Cells were grown at $18^{\circ} \mathrm{C}$ for $50 \mathrm{~h}$ before harvesting and stored at $-80^{\circ} \mathrm{C}$ until further use. Cells were disrupted by microfluidization (M-110S Microfluidizer) (50 mM HEPES pH7.5, $100 \mathrm{mM}$ $\mathrm{NaCl}, 10 \mathrm{mM}$ Imidazole) and soluble protein was isolated by ultracentrifugation at $50000 \mathrm{~g}$ for $30 \mathrm{~min}$. His-hTGT was loaded onto His-Talon Superflow (GE Healthcare) and washed with additional $1 \mathrm{M} \mathrm{LiCl}$. Target protein was eluted in a $20 \mathrm{CV}$ gradient to $50 \mathrm{mM}$ HEPES pH7.5, $100 \mathrm{mM} \mathrm{NaCl}, 500 \mathrm{mM}$ Imidazole. Protein was further purified by Superdex S200 (GE Healthcare) size exclusion chromatography ( $20 \mathrm{mM}$ HEPES pH 7.5, $100 \mathrm{mM} \mathrm{NaCl}$ ). His-hTGT was concentrated to $16-20 \mathrm{mg} / \mathrm{mL}$ and stored at $-80^{\circ} \mathrm{C}$ until further use.

In vitro transcription and tRNA purification. Saccharomyces pombe tRNA ${ }^{\text {Asp }}$ (sptRNA ${ }^{\text {Asp }}$ ) was transcribed from linearized vector template in run off in vitro transcription. Reaction contained T7 Polymerase and $10 \mathrm{mM}$ rNTPs each in $1 \times$ HT buffer $\left(30 \mathrm{mM}\right.$ HEPES pH 8.0, $25 \mathrm{mM} \mathrm{MgCl}_{2}, 10 \mathrm{mM}$ DTT, $2 \mathrm{mM}$ Spermidine, 
$0.01 \%$ Triton X-100). After incubation for $3-16 \mathrm{~h}$ at $37^{\circ} \mathrm{C}$ reaction was stopped with $50 \mathrm{mM}$ EDTA final, concentrated and run in a 10\% Urea-Polyacrylamid gel. tRNA was cut from the gel, extracted (10 mM Tris/ $\mathrm{HCl} \mathrm{pH8.0}$ $1 \mathrm{mM}$ EDTA, $300 \mathrm{mM} \mathrm{NaCl}$ ) and subjected to ethanol precipitation. Purified tRNA was dissolved in $\mathrm{H}_{2} \mathrm{O}$ and stored at $-20^{\circ} \mathrm{C}$ until further use.

Q-Base synthesis. The queuine base was synthesized either as described previously ${ }^{26}$, or with a modified synthesis strategy for the (1R,2S,3S)-1-bromo-2,3-O-isopropylidene-cyclopent-4-ene building block starting from inexpensive methyl $\alpha$-D-galactopyranoside. This synthesis is described in detail in the supplementary information.

Q-base incorporation. For incorporation of Q-base at position 34, unlabeled or fluorescein labelled sptR$\mathrm{NA}^{\text {Asp }}$, was incubated with 100 fold molar excess of free $\mathrm{Q}$ base and under presence of $0.5 \mu \mathrm{M}$ purified his-hTGT (100 mM HEPES pH 7.5, $20 \mathrm{mM} \mathrm{MgCl}_{2}, 5 \mathrm{mM}$ DTT). After four hours the reaction was precipitated with ethanol and pelleted RNA was resolubilized in $\mathrm{H}_{2} \mathrm{O}$. Unreacted free Q-base was removed by desalting using Zeba spin desalting columns $7 \mathrm{~K}$ MWCO (ThermoFisher Scientific). Q-incorporation was confirmed by a Q-specific band-shift, a method previously described ${ }^{27}$, using denaturing $10 \%$ polyacrylamide gels containing urea and $0.5 \%$ (w/v) 3-aminophenylboronic acid (Sigma Aldrich).

tRNA labelling and affinity measurements. $5 \mathrm{nmol}$ tRNA were incubated $50 \mathrm{~min}$ in $400 \mu \mathrm{L}$ volume, containing $100 \mathrm{mM} \mathrm{NaOAc} \mathrm{pH5.5,} 2.5 \mathrm{mM} \mathrm{KIO}_{4}$ at $4{ }^{\circ} \mathrm{C}$. After ethanol precipitation the labelling reaction was carried out in $100 \mathrm{mM} \mathrm{NaOAc}$ pH 5.5 with $1 \mathrm{mM}$ fluorescein-5-thiosemicarbazide (Sigma Aldrich). RNA was again ethanol precipitated and dissolved in $\mathrm{H}_{2} \mathrm{O}$. Unreacted fluorophore was removed using Zeba spin desalting columns (ThermoFisher Scientific). spDnmt 2 was desalted into assay buffer $20 \mathrm{mM}$ Tris/ $\mathrm{HCl}, 50 \mathrm{mM} \mathrm{NaCl}$ prior to the experiment. Anisotropy measurements were performed using a Fluoromax III (Horiba Jobin Yvon) fluorimeter and data was evaluated with FluorEssence software (Horiba Jobin Yvon).

Methyl transferase assay. spDnmt2 kinetics were investigated using a methyltransferase assay kit with colorimetric detection (Cayman Chemicals). The assay is based on a previously reported detection of formation of the methyltransferase reaction co-product SAH by an enzymatic cascade Dorgan et al. ${ }^{28}$. In brief the SAH produced by de-methylation during the Dnmt2 methyl transfer reaction is cleaved by the SAH nucleosidase producing adenine which then undergoes desamination by the adenine desaminase yielding hypoxanthine. Oxidation of this product by the xanthine oxidase produces ureate and hydrogen peroxide latter of which oxidizes the colorimetric agent 3,5-dichloro-2-hydroxybenzenesulfonic acid. This oxidation is monitored at $515 \mathrm{~nm}$. For performing this assay we prepared the reaction mastermix according to the manufacturers instructions. All

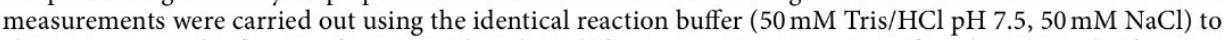
eliminate potential influence of ion strength and $\mathrm{pH}$ differences. $\mathrm{spDnmt} 2$ was transferred into assay buffer using Zeba spin desalting columns $7 \mathrm{~K}$ MWCO (ThermoFisher Scientific) and the concentration was calculated from the absorbtion at $280 \mathrm{~nm}$ using the absorption coefficient of the used spDnmt 2 construct $\left(50880 \mathrm{M}^{-1} \mathrm{~cm}^{-1}\right)$. To check that the assay is conducted under initial rate conditions we measured methyltransferase activity in triplicates with $10 \mu \mathrm{M}$ G34tRNA ${ }^{\text {Asp }}$ for $0.5 \mu \mathrm{M}$ and $0.125 \mu \mathrm{M}$ enzyme yielding a $\Delta \mathrm{A}_{280} / \mathrm{min}$ of $0.01070 \pm 0.00014$ and $0.00267 \pm 0.00021$ respectively after background subtraction. All measurements shown in Fig. 1 were carried out with enzyme derived from the identical purification. $6 \mu \mathrm{L}$ of the desired tRNA concentration were added to $60 \mu \mathrm{L}$ of the mastermix, mixed and preincubated at $20^{\circ} \mathrm{C}$ shortly before measurement $3 \mu \mathrm{L}$ of spDnmt $2.1 \mu \mathrm{M}$ enzyme final were used for measurements with G34tRNA ${ }^{\text {Asp }}$ and $0.5 \mu \mathrm{M}$ final spDnmt 2 concentration for measuring enzyme activity on Q34tRNA Asp to stay within the linear range of the assay. The reaction mixture was mixed and transferred immediately into a quartz cuvette with $10 \mathrm{mM}$ path length (Hellma) for measurement. Change of absorbtion at $515 \mathrm{~nm}$ was monitored over time. Surface treated low binding tips (nerbe plus) were used in all pipetting steps. using an Ultrospec 2100 pro UV/Vis spectrometer (GE Healthcare) until slowing down of the reaction is observed. $\Delta \mathrm{A}_{515 \mathrm{~nm}} / \mathrm{min}$ for each measurement was obtained by fitting an asymptote to the steepest climb using swift II software (Fisher Scientific). Molar activity was calculated using the extinction coefficient of the colorimetric agent $\left(\mathrm{E}_{515 \mathrm{~nm}}=26000 \mathrm{M}^{-1} \mathrm{~cm}^{-1}\right)$. Data were plotted with Origin software (OriginLab) and fitted with the Hill equation $\mathrm{v}=\mathrm{V}_{\max } * \mathrm{x}^{\mathrm{n}} /\left(\mathrm{k}^{\mathrm{n}}+\mathrm{x}^{\mathrm{n}}\right)$ with free $\mathrm{n}$ value.

Crystallization. spDnmt 2 was crystallized using sitting-drop vapor diffusion technique. spDnmt 2 was supplemented with five-fold molar excess of SAH (Sigma Aldrich) and adjusted to $2 \mathrm{mg} / \mathrm{mL}$ protein concentration as determined with Bradford reagent (Bio-Rad). Equal volumes of protein containing solution and crystallization buffer $\left(50 \mathrm{mM}\right.$ MES pH 6.5, $1 \%$ w/v PEG4000, $4 \mathrm{mM} \mathrm{MgCl}_{2}$ ) were mixed. Crystals were obtained after five to seven days at $4^{\circ} \mathrm{C}$. Crystals were cryo-protected by a stepwise increase of glycerol and PEG400 concentration to $15 \%(\mathrm{v} / \mathrm{v})$ each.

Data collection and molecular replacement and refinement. X-ray data collection was performed at $100 \mathrm{~K}$; diffraction images were collected at Petra III beamline P13 at DESY, Hamburg ${ }^{29}$. Diffraction images were indexed, integrated and scaled using the XDS-package ${ }^{30}$. The structure of spDnmt 2 in complex with SAH was solved by molecular replacement by PHASER ${ }^{31}$, as implemented in the CCP4 suite ${ }^{32}$, using the structure of the Entamoebae histolytica Dnmt2 homolog EhMeth (PBD-ID: 3QV2) ${ }^{17}$.

Model building was done with subsequent iterative cycles of automated refinement with PHENIX ${ }^{33}$ and manual model adjustments in $\operatorname{Coot}^{34}$. Crystallographic values are listed in Supplementary Table S1. 
RNA-Protein cross-linking. To reconstitute the tRNA-protein complex, $3.75 \mathrm{nmol}$ Dnmt2 and $3.75 \mathrm{nmol}$ tRNA ${ }^{\text {Asp }}$ were incubated in $100 \mu \mathrm{l} 20 \mathrm{mM}$ HEPES, $50 \mathrm{mM} \mathrm{NaCl} 2 \mathrm{mM}$ DTT, pH 7.5 for $30 \mathrm{~min}$ on ice. One sample containing tRNA ${ }^{\text {Asp }}$ was UV-irradiated at $254 \mathrm{~nm}$ for $10 \mathrm{~min}$, while a second non-irradiated sample was kept as a non-cross-linked control. Further sample processing was performed as described previously ${ }^{35}$. To generate peptides from RNA-complexed proteins, both trypsin and chymotrypsin were used as described in generating peptides from RNA cross-linked protein ${ }^{35}$.

Mass spectrometry (MS/MS) and MS data analysis. Prepared samples were loaded onto a self-packed C18 column, mounted to a Dionex Ultimate 300 UHPLC $^{+}$focused (Thermo Scientific): $3 \mu \mathrm{m}$ pore size, $75 \mu \mathrm{m}$ in diameter, $30 \mathrm{~cm}$ in length (Reprosil-Pur ${ }^{\circledR} 120 \mathrm{C} 18-\mathrm{AQ}$, Dr. Maisch GmbH). Peptides were separated by reverse-phase chromatography on a $58 \mathrm{~min}$ multi-step gradient with a flow rate of $0.3-0.4 \mu \mathrm{L} / \mathrm{min}$ before entering the mass spectrometer (OT Fusion Lumos, Thermo Scientific). MS1 spectra were recorded in profile mode with a resolution of $120 \mathrm{k}$, whereas MS 2 spectra were recorded in centroid mode with a resolution of $30 \mathrm{k}$. The isolation window was set to $1.6 \mathrm{~m} / \mathrm{z}$ and the dynamic exclusion was set to $9 \mathrm{~s}$. Raw data of RNA-protein hetero-conjugates were analysed and manually validated using the OpenMS pipeline RNPxl and OpenMS TOPPASViewer ${ }^{35}$.

Model preparation for use in Rosetta. The crystal structure of spDnmt 2 monomer was subjected to an all-atom refinement with all-heavy-atom constraints in the Rosetta force field using the default Rosetta Relax protocol ${ }^{36}$. Running relax with side-chain and backbone coordinate constraints minimizes Rosetta energy by searching the local conformational space in order to remove clashes and rotamers with unfavorable Rosetta energy, while keeping all non-hydrogen atoms as close as possible to their starting positions in the crystal structure. The relaxation was carried out 20 times and the structure with the lowest energy was selected for in silico docking experiments. tRNA models were extracted from crystal structures (PDB ids: 1VTQ, 1ASZ, 1QF6, 4WJ3, 4WT8) of both unbound and protein-bound tRNA forms and thus representing different conformations of the molecule. These models were converted to a format suitable for Rosetta (all modified bases were replaced by unmodified ones).

Protein-RNA docking experiments. Docking experiments were performed in Rosetta ${ }^{37}$ using the RosettaDock application optimized for predicting RNA-Protein complexes ${ }^{38}$. The used docking protocol was composed of two search stages: low resolution and high resolution. The low resolution stage uses a coarse-grained representation of the ligand and receptor (the side chains are replaced by a single unified pseudo-atom) making it possible to quickly sample the search space for the docking partners. The subsequent high resolution stage rebuilds the all-atom partners from the low resolution candidates to perform the full atom mode refinement including rotamer search and possibly loop optimization.

The low resolution stage was carried out for five tRNA models (PDB ids: 1VTQ, 1ASZ, 1QF6, 4WJ3, 4WT8). In order to fully sample the search space, 50.000 poses were generated for each tRNA model. Favorable initial docking orientations were identified based on the total Rosetta score as well as I_sc score (representing the energy of the interactions across the interface) and validated by the experimental crosslinks and distance constraints defined between the phosphate group of the modified nucleotide 38 and spDnmt2 active site residues These active site residues have been identified based on superposition of the receptor (spDnmt 2 crystal structure) with the crystal structure of Dnmt 1 complexed with DNA ${ }^{18}$. The best scoring pose (low resolution model) was obtained for three-dimensional structure of yeast tRNA-ASP, PDB id: 1VTQ. This low resolution model has been subjected to the high resolution docking protocol (10.000 poses). Due to some short non-bonded interactions between the docked tRNA model and the receptor molecule, the high resolution docking step resulted in a positional shift of the tRNA molecule away from the active site (rmsd calculated for all phosphate atoms $\sim 3 \AA$ ). In order to obtain a complete atomic model of the docked spDnmt2-tRNA complex satisfying both the experimental crosslinks and positional constraints (nucleotide 38 should be positioned close to the flipped nucleotide in the Dnmt1-DNA complex structure), we superimposed the IVTQ structure onto the best low resolution model, thus skipping the high resolution docking step. Modeling of the flipped 38 nucleotide as well as building queuing modification at position 34 was performed in Coot.

Data availability. Coordinates and structure factors have been deposited within the Protein Data Bank (PDB code $6 \mathrm{FDF}$ ). All other datasets generated during the current study are available from the corresponding author on reasonable request.

\section{References}

1. Machnicka, M. A. et al. MODOMICS: a database of RNA modification pathways-2013 update. Nucleic Acids Res 41, D262-267, https://doi.org/10.1093/nar/gks1007 (2013)

2. Rozenski, J., Crain, P. F. \& McCloskey, J. A. The RNA Modification Database: 1999 update. Nucleic Acids Res 27, $196-197$ gkc060 (1999).

3. Gu, C., Begley, T. J. \& Dedon, P. C. tRNA modifications regulate translation during cellular stress. FEBS Lett https://doi.org/10.1016/j. febslet.2014.09.038 (2014).

4. Helm, M. Post-transcriptional nucleotide modification and alternative folding of RNA. Nucleic Acids Res 34, 721-733, https://doi. org/10.1093/nar/gkj471 (2006).

5. Motorin, Y. \& Helm, M. tRNA stabilization by modified nucleotides. Biochemistry 49, 4934-4944, https://doi.org/10.1021/bi100408z (2010).

6. Schaefer, M. et al. RNA methylation by Dnmt2 protects transfer RNAs against stress-induced cleavage. Genes Dev 24, 1590-1595, https://doi.org/10.1101/gad.586710 (2010).

7. Durdevic, Z., Mobin, M. B., Hanna, K., Lyko, F. \& Schaefer, M. The RNA methyltransferase Dnmt2 is required for efficient Dicer-2 dependent siRNA pathway activity in Drosophila. Cell Rep 4, 931-937, https://doi.org/10.1016/j.celrep.2013.07.046 (2013).

8. Shanmugam, R. et al. Cytosine methylation of tRNA-Asp by DNMT2 has a role in translation of proteins containing poly-Asp sequences. Cell Discov 1, 15010, https://doi.org/10.1038/celldisc.2015.10 (2015).

9. Tuorto, F. et al. The tRNA methyltransferase Dnmt2 is required for accurate polypeptide synthesis during haematopoiesis. EMBO J 34, 2350-2362, https://doi.org/10.15252/embj.201591382 (2015). 
10. Muller, M. et al. Dynamic modulation of Dnmt2-dependent tRNA methylation by the micronutrient queuine. Nucleic Acids Res 43, 10952-10962, https://doi.org/10.1093/nar/gkv980 (2015).

11. Harada, F. \& Nishimura, S. Possible anticodon sequences of tRNA His, tRNAAsm, and tRNA Asp from Escherichia coli B. Universal presence of nucleoside Q in the first postion of the anticondons of these transfer ribonucleic acids. Biochemistry 11, 301-308 (1972).

12. Fergus, C., Barnes, D., Alqasem, M. A. \& Kelly, V. P. The queuine micronutrient: charting a course from microbe to man. Nutrients 7, 2897-2929, https://doi.org/10.3390/nu7042897 (2015).

13. Rozov, A., Demeshkina, N., Westhof, E., Yusupov, M. \& Yusupova, G. Structural insights into the translational infidelity mechanism. Nat Commun 6, 7251, https://doi.org/10.1038/ncomms8251 (2015).

14. Romier, C., Reuter, K., Suck, D. \& Ficner, R. Crystal structure of tRNA-guanine transglycosylase: RNA modification by base exchange. EMBO J 15, 2850-2857 (1996).

15. Chen, Y. C., Kelly, V. P., Stachura, S. V. \& Garcia, G. A. Characterization of the human tRNA-guanine transglycosylase: confirmation of the heterodimeric subunit structure. RNA 16, 958-968, https://doi.org/10.1261/rna.1997610 (2010).

16. Dong, A. et al. Structure of human DNMT2, an enigmatic DNA methyltransferase homolog that displays denaturant-resistant binding to DNA. Nucleic Acids Res 29, 439-448 (2001).

17. Schulz, E. C., Roth, H. M., Ankri, S. \& Ficner, R. Structure analysis of Entamoeba histolytica DNMT2 (EhMeth). Plos One 7, e38728, https://doi.org/10.1371/journal.pone.0038728 (2012).

18. Song, J., Teplova, M., Ishibe-Murakami, S. \& Patel, D. J. Structure-based mechanistic insights into DNMT1-mediated maintenance DNA methylation. Science 335, 709-712, https://doi.org/10.1126/science.1214453 (2012).

19. Jurkowski, T. P., Shanmugam, R., Helm, M. \& Jeltsch, A. Mapping the tRNA binding site on the surface of human DNMT2 methyltransferase. Biochemistry 51, 4438-4444, https://doi.org/10.1021/bi3002659 (2012).

20. Porter, C. M. \& Miller, B. G. Cooperativity in monomeric enzymes with single ligand-binding sites. Bioorg Chem 43, 44-50, https:// doi.org/10.1016/j.bioorg.2011.11.001 (2012).

21. Jurkowski, T. P. \& Jeltsch, A. On the evolutionary origin of eukaryotic DNA methyltransferases and Dnmt2. Plos One 6, e28104, https://doi.org/10.1371/journal.pone.0028104 (2011).

22. Fischer, M. New aspects of radionuclide therapy in metastatic bone pain. Anticancer Res 20, 5183-5189 (2000).

23. Mallis, R. J., Brazin, K. N., Fulton, D. B. \& Andreotti, A. H. Structural characterization of a proline-driven conformational switch within the Itk SH2 domain. Nature Structural Biology 9, 900-905, https://doi.org/10.1038/nsb864 (2002).

24. Grathwohl, C. \& Wuthrich, K. Nmr-Studies of the Rates of Proline Cis-Trans Isomerization in Oligopeptides. Biopolymers 20 , 2623-2633, https://doi.org/10.1002/bip.1981.360201209 (1981).

25. Goll, M. G. et al. Methylation of tRNAAsp by the DNA methyltransferase homolog Dnmt2. Science 311, 395-398, https://doi. org/10.1126/science.1120976 (2006).

26. Gerber, H. D. \& Klebe, G. Concise and efficient syntheses of preQ1 base, Q base, and (ent)-Q base. Org Biomol Chem 10, 8660-8668, https://doi.org/10.1039/c2ob26387d (2012).

27. Igloi, G. L. \& Kossel, H. Affinity electrophoresis for monitoring terminal phosphorylation and the presence of queuosine in RNA. Application of polyacrylamide containing a covalently bound boronic acid. Nucleic Acids Res 13, 6881-6898 (1985).

28. Dorgan, K. M. et al. An enzyme-coupled continuous spectrophotometric assay for S-adenosylmethionine-dependent methyltransferases. Anal Biochem 350, 249-255, https://doi.org/10.1016/j.ab.2006.01.004 (2006).

29. Cianci, M. et al. P13, the EMBL macromolecular crystallography beamline at the low-emittance PETRA III ring for high- and lowenergy phasing with variable beam focusing. J Synchrotron Radiat 24, 323-332, https://doi.org/10.1107/S1600577516016465 (2017).

30. Kabsch, W. XDS. Acta Crystallogr D Biol Crystallogr 66, 125-132, https://doi.org/10.1107/S0907444909047337 (2010).

31. McCoy, A. J. et al. Phaser crystallographic software. J Appl Crystallogr 40, 658-674, https://doi.org/10.1107/S0021889807021206 (2007).

32. Winn, M. D. et al. Overview of the CCP4 suite and current developments. Acta Crystallogr D Biol Crystallogr 67, 235-242, https:// doi.org/10.1107/S0907444910045749 (2011).

33. Adams, P. D. et al. PHENIX: a comprehensive Python-based system for macromolecular structure solution. Acta Crystallogr D Biol Crystallogr 66, 213-221, https://doi.org/10.1107/S0907444909052925 (2010).

34. Emsley, P., Lohkamp, B., Scott, W. G. \& Cowtan, K. Features and development of Coot. Acta Crystallogr D Biol Crystallogr 66, 486-501, https://doi.org/10.1107/S0907444910007493 (2010).

35. Kramer, K. et al. Photo-cross-linking and high-resolution mass spectrometry for assignment of RNA-binding sites in RNA-binding proteins. Nat Methods 11, 1064-1070, https://doi.org/10.1038/nmeth.3092 (2014).

36. Nivon, L. G., Moretti, R. \& Baker, D. A Pareto-Optimal Refinement Method for Protein Design Scaffolds. Plos One 8, https://doi. org/10.1371/journal.pone.0059004 (2013).

37. Chaudhury, S. et al. Benchmarking and analysis of protein docking performance in Rosetta v3.2. PLoS One 6, e22477, https://doi. org/10.1371/journal.pone.0022477 (2011).

38. Guilhot-Gaudeffroy, A., Froidevaux, C., Aze, J. \& Bernauer, J. Protein-RNA complexes and efficient automatic docking: expanding RosettaDock possibilities. Plos One 9, e108928, https://doi.org/10.1371/journal.pone.0108928 (2014).

\section{Acknowledgements}

The synchrotron MX data was collected at beamline P13 operated by EMBL Hamburg at the PETRA III storage ring (DESY, Hamburg, Germany). We would like to thank Isabel Bento for the assistance in using the beamline. We further thank Martin Müller and Ann Ehrenhofer-Murray for providing the cDNA of spDnmt2 and we appreciate the advice of Guiseppe Randelli and Claudia Höbartner (University of Würzburg) during RNA purification. We acknowledge funding by the DFG (priority program SPP1784, INST 186/814-1 and INST $186 / 1117-1$ ) to R.F. Parts of the project were supported by a grant to H.U. (DFG SFB860).

\section{Author Contributions}

S.J. and R.F. designed the experiments. S.J. performed cloning and purification of proteins and tRNA as well as data acquisition at synchrotron and determined the structure. H.-D.G. synthesized the Q-base. M.K. did the herein described novel synthesis of queuine under supervision by U.D. A.W. and L.M.W. performed and analyzed the cross-link experiments under supervision by H.U. P.N. conducted docking experiments. Data were interpreted by S.J., P.N. and R.F. The manuscript was prepared by S.J. and R.F.

\section{Additional Information \\ Supplementary information accompanies this paper at https://doi.org/10.1038/s41598-018-27118-5. \\ Competing Interests: The authors declare no competing interests.}

Publisher's note: Springer Nature remains neutral with regard to jurisdictional claims in published maps and institutional affiliations. 
(c) (i) Open Access This article is licensed under a Creative Commons Attribution 4.0 Internationa EY License, which permits use, sharing, adaptation, distribution and reproduction in any medium or format, as long as you give appropriate credit to the original author(s) and the source, provide a link to the Cre ative Commons license, and indicate if changes were made. The images or other third party material in this article are included in the article's Creative Commons license, unless indicated otherwise in a credit line to the material. If material is not included in the article's Creative Commons license and your intended use is not permitted by statutory regulation or exceeds the permitted use, you will need to obtain permission directly from the copyright holder. To view a copy of this license, visit http://creativecommons.org/licenses/by/4.0/.

(c) The Author(s) 2018 


\section{Supporting information}

\section{Title}

Structural insights into the stimulation of S.pombe Dnmt2 catalytic efficiency by the tRNA nucleoside queuosine

\section{Authors}

Sven Johannsson ${ }^{1}$, Piotr Neumann ${ }^{1}$, Alexander Wulf², Luisa M. Welp², Hans-Dieter Gerber $^{3}$, Matthias Krull ${ }^{4}$, Ulf Diederichsen ${ }^{4}$, Henning Urlaub ${ }^{2,5}$ and Ralf Ficner ${ }^{1^{*}}$

\section{Author affiliations}

${ }^{1}$ Department of Molecular Structural Biology, Institute of Microbiology and Genetics, GZMB, GeorgAugust-University Göttingen, 37077 Göttingen, Germany

2 Bioanalytical Mass Spectrometry Research Group, Max Planck Institute for Biophysical Chemistry, Am Faßberg 11, 37077 Göttingen, Germany;

${ }^{3}$ Institut für Pharmazeutische Chemie der Philipps-Universität Marburg, Marbacher Weg 6, 35032 Marburg, Germany.

${ }^{4}$ Institut für Organische und Biomolekulare Chemie, Georg-August-Universität Göttingen, Tammannstrasse 2, 37077, Göttingen, Germany.

${ }^{5}$ Bioanalytics, Department of Clinical Chemistry, University Medical Center Göttingen, Robert-KochStraße 40, 37075 Göttingen, Germany

* To whom correspondence should be addressed. Tel: +49 551 3914072; Email: rficner@uni-goettingen.de 


\section{Supplementary Table S1.}

Crystallographic statistics of the spDnmt2 crystal structure.

\begin{tabular}{|c|c|}
\hline \multicolumn{2}{|l|}{ Data Collection } \\
\hline X-ray source & $\begin{array}{l}\text { Synchrotron (PETRA III, } \\
\text { P13) }\end{array}$ \\
\hline Detector & Dectris Pilatus 6M-F \\
\hline Wavelength $(\AA)$ & 1.033 \\
\hline Space group & $\mathrm{C} 2$ \\
\hline \multicolumn{2}{|l|}{ Cell dimensions } \\
\hline$a, b, c(\AA)$ & $153.82,114.78,113.76$ \\
\hline$\alpha, \beta, \gamma\left({ }^{\circ}\right)$ & $90.00,131.05,90.00$ \\
\hline Resolution $(\AA)$ & $40.80-1.70(1.80-1.70)$ \\
\hline $\mathrm{R}_{\text {meas }}(\%)$ & $3.5(59.8)$ \\
\hline $1 / \sigma(\mathrm{l})$ & $20.63(2.69)$ \\
\hline $\mathrm{CC}_{1 / 2}$ & $99.9(81.3)$ \\
\hline Completeness (\%) & $99.0(90.3)$ \\
\hline Redundancy & $3.49(3.57)$ \\
\hline \multicolumn{2}{|l|}{ Refinement } \\
\hline Resolution $(\AA)$ & $30.0-1.7$ \\
\hline No. reflections & 162230 \\
\hline $\mathrm{R}_{\text {work }}(\%)$ & 17.39 \\
\hline$R_{\text {free }}(\%)$ & 19.48 \\
\hline \multicolumn{2}{|l|}{ No. of atoms } \\
\hline Protein & 10490 \\
\hline Water & 832 \\
\hline Ligand & 170 \\
\hline Ions & 0 \\
\hline \multicolumn{2}{|l|}{ B-factors $\left(\AA^{2}\right)$} \\
\hline Protein & 44.3 \\
\hline Ligand & 51.0 \\
\hline Water and other small molecules & 49.6 \\
\hline \multicolumn{2}{|l|}{ R.m.s. deviations } \\
\hline Bond lengths $(\AA)$ & 0.005 \\
\hline Bond angles $\left({ }^{\circ}\right)$ & 0.797 \\
\hline \multicolumn{2}{|l|}{ MolProbity analysis } \\
\hline Ramachandran favoured (\%) & 96.94 \\
\hline Ramachandran outliers (\%) & 0.00 \\
\hline Rotamer outliers (\%) & 1.80 \\
\hline Clashscore & 3.78 \\
\hline
\end{tabular}




\section{Supplementary Table S2.}

Molar activity of spDnmt2 of the indicated final concentration of either G34tRNA ${ }^{\text {Asp }}$ (left) or Q34tRNA ${ }^{\text {Asp }}$. Activity in $U$ per enzyme concentration is calculated as mean values from three independent replicates. $U$ is defined as substrate conversion in $\mu \mathrm{mol} / \mathrm{min}$. SD indicates standard deviation. Figure 1 represents a graphical representation of these values.

\begin{tabular}{|l|l|l|}
\hline $\begin{array}{c}\mu \mathrm{M} \\
\mathbf{G 3 4 t R N A}^{\text {Asp }}\end{array}$ & $\mathbf{U} / \boldsymbol{\mu m o l}$ & SD \\
\hline 49.304 & 1.222 & 0.010 \\
\hline 32.870 & 1.122 & 0.054 \\
\hline 16.435 & 0.715 & 0.035 \\
\hline 12.326 & 0.578 & 0.019 \\
\hline 8.217 & 0.369 & 0.006 \\
\hline 6.574 & 0.283 & 0.037 \\
\hline 4.930 & 0.187 & 0.004 \\
\hline 2.465 & 0.069 & 0.004 \\
\hline
\end{tabular}

\begin{tabular}{|l|l|l|}
\hline $\begin{array}{c}\boldsymbol{\mu} \mathbf{M} \\
\text { Q34tRNA }^{\text {Asp }}\end{array}$ & \multicolumn{1}{|c|}{$\boldsymbol{U / \mu \mathrm { mol }}$} & \multicolumn{1}{c|}{ SD } \\
\hline 23.212 & 2.078 & 0.012 \\
\hline 15.475 & 1.958 & 0.023 \\
\hline 7.737 & 1.532 & 0.044 \\
\hline 5.803 & 1.163 & 0.004 \\
\hline 4.836 & 0.999 & 0.032 \\
\hline 3.869 & 0.878 & 0.036 \\
\hline 2.321 & 0.491 & 0.018 \\
\hline 1.161 & 0.296 & 0.008 \\
\hline
\end{tabular}




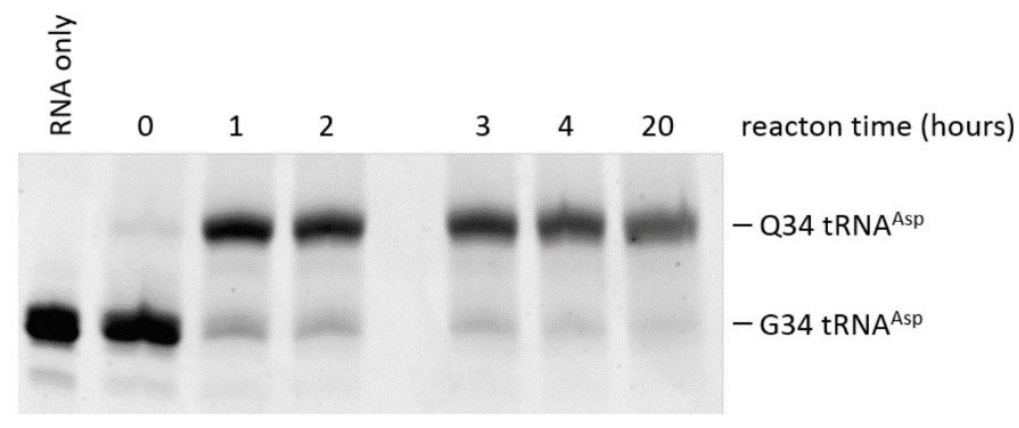

Supplementary Figure S1. Incorporation of queuine into tRNA Asp by human TGT tRNA $^{\text {Asp }}$ was incubated with free queuine and TGT samples were taken from the reaction after addition of recombinant TGT at the indicated time and resolved on 3phenylboronic acid containing denaturing $10 \%$ polyacrylamide gel. 

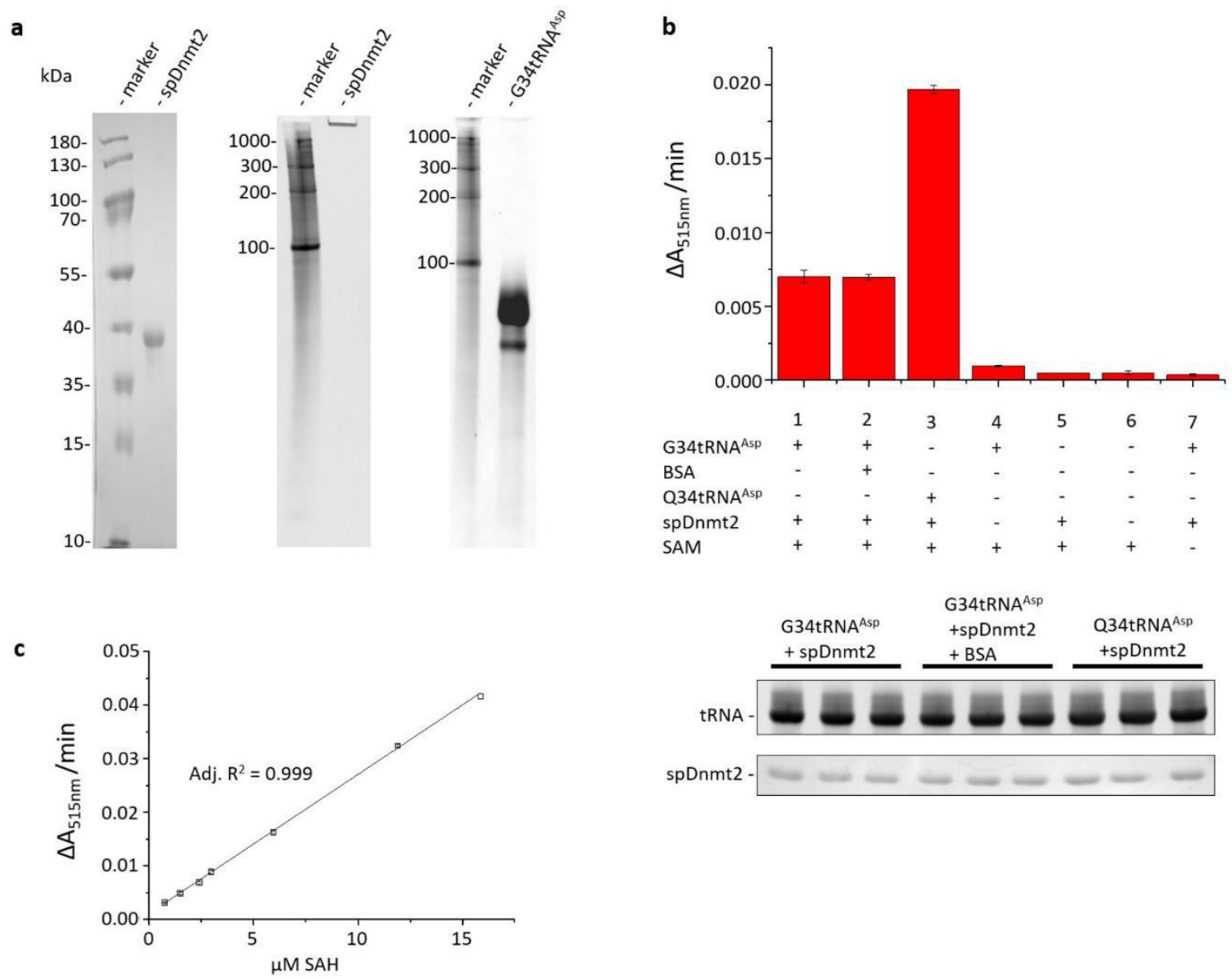

(n)

\section{Supplementary Figure S2. Methyltransferase assay supporting data}

(a) (right) Denaturing gel analysis of spDnmt2 as the enzyme is used in the assay. The left lane received $5 \mu \mathrm{L}$ Prestained Protein Ladder (Fermentas). Sizes of bands in kilo Daltons are indicated on the left. $1 \mu \mathrm{g}$ purified spDnmt2 protein was loaded in the right lane. (middle) Denaturing $8 \mathrm{M}$ Urea containing gel with $10 \%$ Polyacrylamide. Left lane received $5 \mu \mathrm{L}$ of Ribo Ruler Low Range RNA ladder (Fermentas), which equals to $175 \mu \mathrm{g}$ per band. Band sizes in bases are indicated on the left. $5 \mu \mathrm{g}$ purified spDnmt2 was loaded in the right lane. (right) Denaturing $8 \mathrm{M}$ Urea containing gel with $10 \%$ Polyacrylamide. Left lane received $5 \mu \mathrm{L}$ of Ribo Ruler Low Range RNA ladder (Fermentas). Purified G34tRNAAsp was loaded in the right lane. (b) The methyltransferase assay was performed as described in the materials but with different combinations of components. Indicated measurements received $5.5 \mu \mathrm{M}$ G34tRNA Asp Q34tRNA Asp and/or $1 \mu \mathrm{M}$ spDnmt2 final concentrations. Change in absorbance was measured at $515 \mathrm{~nm}$ over time. Every measurement was carried out as three 
independent triplicates. Error bars indicate standard deviation. No change in reaction velocity was observed for spDnmt2 activity on G34tRNA ${ }^{\text {Asp }}$ when reaction tubes were coated for several minutes with $0.1 \mathrm{M} \mathrm{BSA}$ prior to mixing of the components. spDnmt2 exhibits almost three-fold higher activity on the queuine modified compared to the unmodified tRNA at this substrate concentration. Below, equal use of tRNA and Dnmt2 in reaction triplicates is shown. Samples were taken after monitoring the reaction and mixed in equal amounts with 2x RNA loading dye or 2x SDS loading dye. equal volumes of $8 \mu \mathrm{L}$ per sample or $10 \mu \mathrm{L}$ per lane were loaded on a denaturing $10 \%$ polyacrylamide Urea gel or $12.5 \%$ polyacrylamide SDS-PAGE gel respectively. The Urea and SDS containing gels were stained with GelRed (Biotinum) or comassie respectively for visualization. (c) Overview of increasing $\triangle A_{515 n m} / \mathrm{min}$ with increasing concentrations of SAH. Reaction mixture did not receive RNA or protein but the indicated final concentration of SAH. Linear regression shows the linear dependency of change in absorbtion at $515 \mathrm{~nm}$ with increasing concentration of SAH within the measured assay range. 
a

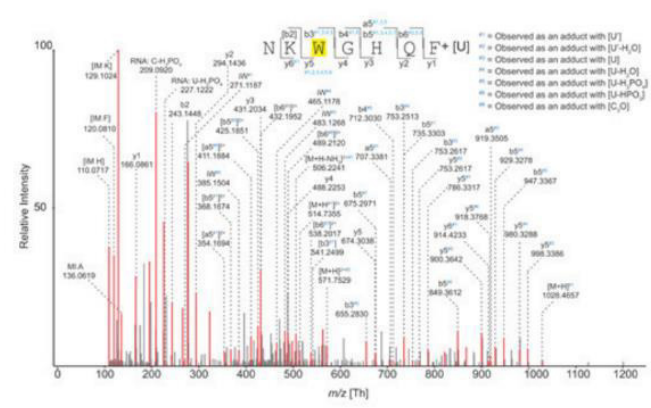

C

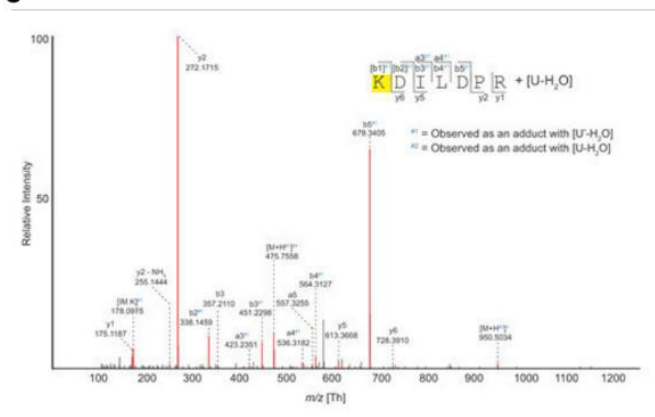

b

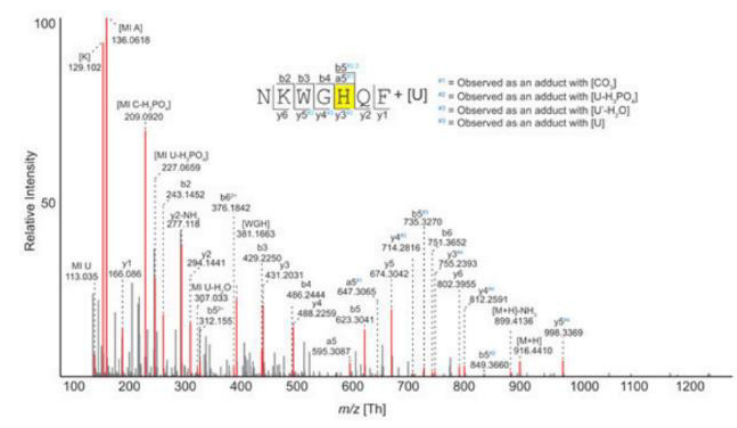

d

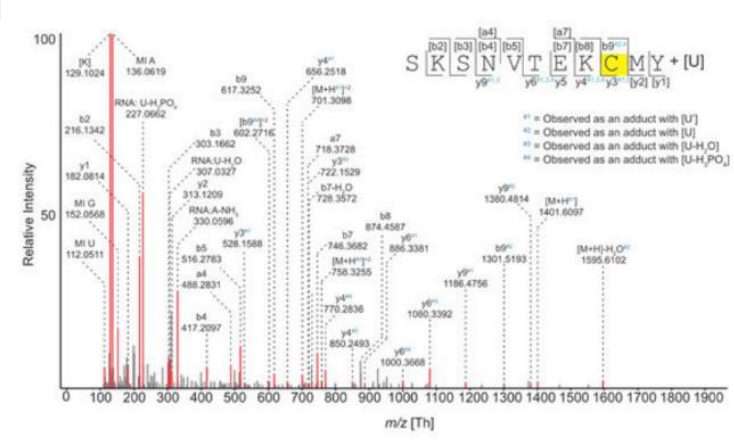

Supplementary Figure S3. MS/MS spectra of RNA (oligo) nucleotides crosslinked to spDnmt2 peptides identified for the tRNA ${ }^{\text {Asp }}$-Dnmt2 complex

(a) The chymotryptic heptapeptide $\mathrm{N}_{219}-\mathrm{F}_{225}$ cross-linked to a uracil nucleotide. The identification of both shifted $y$-ions and multiple b-ions and in addition to a critical y5/ b3 shifted ion pair, unambiguously assigns the location of the cross-link to $W_{221}$ (highlighted in yellow). (b) The same chymotryptic heptapeptide as in (a) was found to be cross-linked to a uracil nucleotide. In this case, $\mathrm{H}_{223}$ is the cross-linked amino acid as revealed by the shifted $\mathrm{y} 3, \mathrm{y} 4, \mathrm{y} 5$ and b5 fragment ions. (c) The tryptic heptapeptide $\mathrm{K}_{91}-\mathrm{R}_{97}$ was also found to be cross-linked to a uracil nucleotide, as identified by an almost complete shifted $b$-ion series starting at b1 in addition to shifted a3 and a4 ions ions. $\mathrm{K}_{91}$ was identified to be the cross-linking amino acid. (d) For the chemotryptic peptide $S_{295}-Y_{305}$, a cysteine residue was identified to be cross-linked to uracil. Shifted $y$-ions originating at the $y 3$ ion, and the shifted b9-ion identify $\mathrm{C}_{303}$ as the cross-linked amino acid. Ions with a mass shift of $\#^{1}, \#^{2}, \#^{3}, \#^{4}, \#^{5}, \#^{6}$ and $\#^{7}$ correspond to the cross-linked nucleotides U', U'- $\mathrm{H}_{2} \mathrm{O}, \mathrm{U}, \mathrm{U}-\mathrm{H}_{2} \mathrm{O}, \mathrm{U}-\mathrm{H}_{3} \mathrm{PO}_{4}, \mathrm{U}-\mathrm{HPO}_{3}$ and $\mathrm{CO}_{3}$, respectively. U: Uracil (324.04 Da); U': nucleobase of U (112.02 Da). 


\section{Synthesis of queuine}

The reported synthetic routs for the preparation of queuine ${ }^{1-5}$ require a huge synthetic effort and are based on expensive or toxic reagents. Furthermore, current syntheses afford stereoselective conversions. Gerber and Klebe reported a queuine synthesis based on fragment coupling of 7-aminoethyl-7-deazaguanine (preQ) and (1R,2S,3S)1-bromo-2,3-O-isopropylidene-cyclopent-4-ene building blocks ${ }^{6}$. Based on this strategy, herein a new route to the (1R,2S,3S)-1-bromo-2,3-O-isopropylidenecyclopent-4-ene building block is described starting from inexpensive methyl a-Dgalactopyranoside. Thereby, formation of the chiral centers by stereoselective reactions and usage of toxic pyridinium chlorochromate was avoided. $\alpha-D-$ galactopyranoside already contains the configurational pattern for the hydroxyl groups at positions 2, 3 and 4 of the cyclopentene building block used in the Q-base synthesis. The $\alpha$-D-galactopyranoside configuration was conserved, suitable protecting groups were applied and enantioselective reactions made expendable.

As outlined in supplementary figure S3 the hydroxyl groups at C2, C3 and C4 of a-Dgalactopyranoside (1) were protected as acetal and silyl ether, respectively, and the primary alcohol was converted into the iodide. Reduction of sugar 3 at C5 and C6 with zinc provided the terminal olefin and the aldehyde in intermediate 4. After conversion of the aldehyde to a second terminal olefin, ring closing metatheses yielded cyclopenteneol 7, which was then brominated to give (1R,2S,3S)-1-bromo-2,3-Oisopropylidene-cyclopent-4-ene (8) as the desired building block.

Compounds 2, 3, and 4 were synthesized following slightly modified protocols from Moynihan et al. ${ }^{7}$ Compound 7 was synthesized following a modified protocol from Smith et al. ${ }^{8}$ Compounds 8, 10, and 12 were synthesized following a protocol developed by Gerber et al. ${ }^{6}$.

\section{General Reagent Information}

All reagents, including anhydrous solvents, were purchased from commercial suppliers (ABCR, Acros-Organics, Alfa Aesar, Fisher Scientific, Merck, Roth, Sigma-Aldrich, TCI and $V W R$ ) were used without further purification. Technical solvents were distilled prior to use. Water was purified using a Simplicity water purification system from Millipore. 


\section{General Experimental Methods}

NMR spectra were recorded at Varian instruments (Mercury 300, Mercury VX 300, Unity 300, VNMRS-300) and Bruker instruments (Avance III 400, Avance III HD 400) using chloroform- $d$ or methanol- $d_{4}$ as the internal standard. Chemical shifts are quoted in ppm. Multiplicities are abbreviated as follows: $s=$ singlet, $d=$ doublet, $d d=$ doublet of doublets, $\mathrm{t}=$ triplet, $\mathrm{dt}=$ doublet of triplets, $\mathrm{m}=$ multiplet, $\mathrm{dm}=$ doublet of multiplets.

High resolution ESI spectra were obtained with a Bruker APEX-Q IV $7 T$ spectrometer. The values are given as $\mathrm{m} / \mathrm{z}$ relation.

Reactions were monitored by thin-layer chromatography (TLC) carried out on aluminium backed plates of silica gel $60 \mathrm{~F}_{254}$ (layer thickness: $0.20 \mathrm{~mm}$ ) from Merck. Spots were detected by fluorescence quenching at $254 \mathrm{~nm}$ or dying with $\mathrm{KMnO}_{4}$ solution. Purification by flash chromatography was conducted using Merck Silica Gel 60 (particle size: 40-62 $\mu \mathrm{m}$ ). 

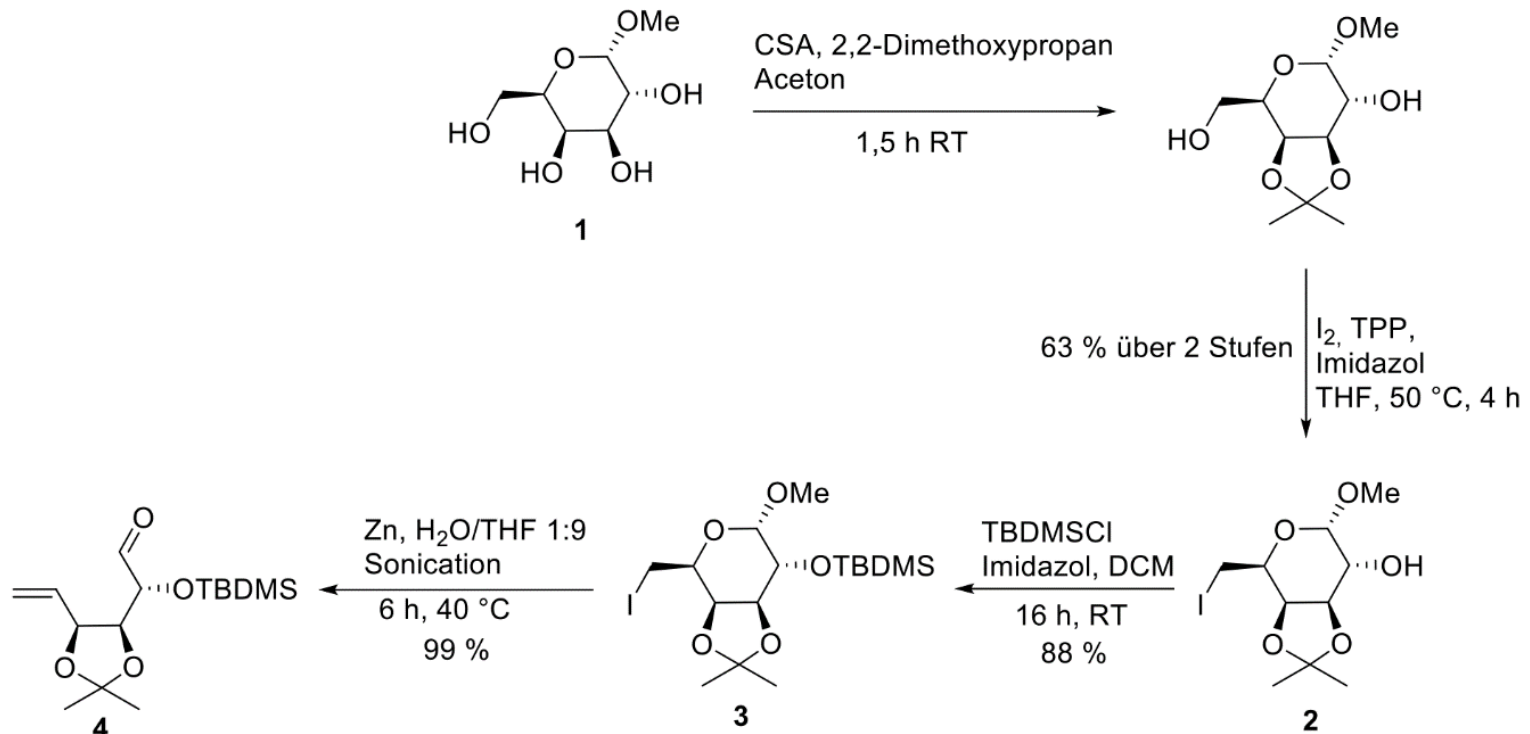

$$
67 \% \mid \begin{aligned}
& \text { Tebbe Reagent } \\
& \text { THF, } 0{ }^{\circ} \mathrm{C}, 15 \mathrm{~min}
\end{aligned}
$$

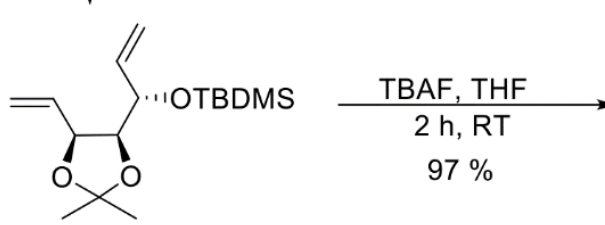

5

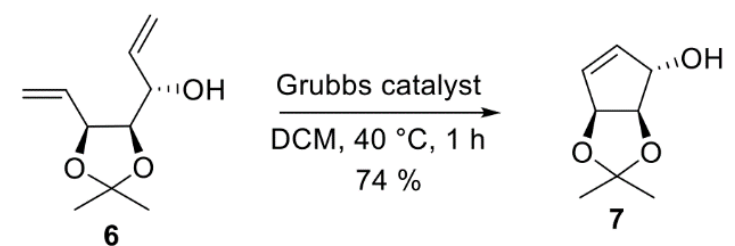

6<smiles>CC1(C)O[C@H]2C=C[C@@H](NCc3c[nH]c4nc(N)[nH]c(=O)c34)[C@H]2O1</smiles>

10

$96 \%$

$\mathrm{HCl} / \mathrm{MeOH}$ reflux, $3 \mathrm{~h}$<smiles></smiles>

Supplementary Figure S4. Synthetic route to queuine (11) 


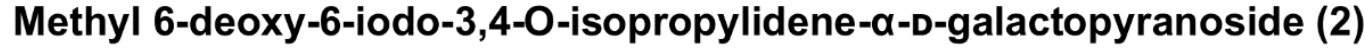

To a stirred suspension of methyl $\alpha$-D-galactopyranoside (1) (25 g, $129 \mathrm{mmol}, 1.0$ eq.) in acetone (450 mL), 2,2-dimethoxypropane (39,5 mL, $233 \mathrm{mmol}, 2.5$ eq.) and camphor sulfonic acid ( $5 \mathrm{~mol} \%, 1.5 \mathrm{~g}, 6.45 \mathrm{mmol})$ were added and the resulting solution was stirred for $2 \mathrm{~h}$ at ambient temperature. After addition of $\mathrm{NEt}_{3}(3 \mathrm{~mL})$ the mixture was evaporated in vacuo and the resulting residue was resolved in THF $(900 \mathrm{~mL})$. Triphenylphosphine (50.75 g, $194 \mathrm{mmol}, 1.5 \mathrm{eq}$.$) and imidazole (17.6 g,$ $258 \mathrm{mmol}, 2.0$ eq.) were added and the solution was heated to $50^{\circ} \mathrm{C}$. Subsequently a solution of iodine (49.2 g, $194 \mathrm{mmol}, 1.5 \mathrm{eq}$ ) in THF (180 mL) was added dropwise and the solution was stirred for $2.5 \mathrm{~h}$ at $50^{\circ} \mathrm{C}$. The solution was cooled to ambient temperature and quenched with aqueous $\mathrm{Na}_{2} \mathrm{~S}_{2} \mathrm{O}_{3}$ solution $(10 \%, 600 \mathrm{~mL})$. The layers were separated, and the aqueous layer was extracted with EtOAc ( $3 \times 200 \mathrm{~mL})$. The combined organic layers were washed with sat. aqueous $\mathrm{NaCl}$ solution $(2 \times 200 \mathrm{~mL})$, dried over $\mathrm{MgSO}_{4}$ and the solvents were evaporated in vacuo. The crude product was purified by column chromatography on silica gel using pentane/EtOAc 1:1. The desired product 2 (27.9 g, $81.1 \mathrm{mmol}, 63 \%$ over two steps) was obtained as a white solid.

${ }^{1} \mathrm{H}-\mathrm{NMR}\left(\mathrm{CDCl}_{3}, 300 \mathrm{MHz}\right): \delta=4.68(\mathrm{~d}, \mathrm{~J}=3.9 \mathrm{~Hz}, 1 \mathrm{H}, \mathrm{H}-1), 4.25-4.17(\mathrm{~m}, 2 \mathrm{H}, \mathrm{H}-3$, $H-4)$, 4.08-4.00 (m, $1 \mathrm{H}, \mathrm{H}-5), 3.81-3.75(\mathrm{~m}, 1 \mathrm{H}, \mathrm{H}-6), 3.43$ (s, $\left.3 \mathrm{H}, \mathrm{OCH}_{3}\right), 3.30-3.17$ (m, $2 \mathrm{H}, \mathrm{H}-6, \mathrm{H}-6$ '), 1.40 (s, $\left.3 \mathrm{H}, \mathrm{C}\left(\mathrm{CH}_{3}\right)_{2}\right), 1.26\left(\mathrm{~s}, 3 \mathrm{H}, \mathrm{C}\left(\mathrm{CH}_{3}\right)_{2}\right) \mathrm{ppm}$.

${ }^{13} \mathrm{C}-N M R\left(\mathrm{CDCl}_{3}, 126 \mathrm{MHz}\right): \delta=109.7\left(C\left(\mathrm{CH}_{3}\right)_{2}\right), 98.2(C-1), 75.8(C-3), 73.5(C-4)$, $69.4(\mathrm{C}-5), 68.6(\mathrm{C}-2), 55.6\left(\mathrm{OCH}_{3}\right), 27.4\left(\mathrm{C}_{\left.\left(\mathrm{CH}_{3}\right)_{2}\right),} 25.7\left(\mathrm{C}\left(\mathrm{CH}_{3}\right)_{2}\right), 2.8(\mathrm{C}-6) \mathrm{ppm}\right.$.

MS (ESI): $\mathbf{m} / \mathbf{z}(\%)=367.0(100)[\mathrm{M}+\mathrm{Na}]^{+}$.

HRMS (ESI): calc. for $\mathrm{C}_{10} \mathrm{H}_{17} \mathrm{IO}_{5} \mathrm{Na}[\mathrm{M}+\mathrm{Na}]^{+}: 367.0013$, found 367.0017 .

\section{Methyl 6-deoxy-6-iodo-3,4-0-isopropylidene-2-0-tertbutyldimethylsilyl-a-D- galactopyrano-side (3)}

Under argon atmosphere iodide $2(10.0 \mathrm{~g}, 29.1 \mathrm{mmol}, 1.0$ eq.), imidazole (2.38 g, $34.9 \mathrm{mmol}, 1.2$ eq.) and TBDMSCI (5.26 g, $34.9 \mathrm{mmol}, 1.2 \mathrm{eq}$.$) were dissolved in dry$ DCM $(120 \mathrm{~mL})$ and the solution was stirred for $16 \mathrm{~h}$ at ambient temperature. The reaction was quenched with water $(100 \mathrm{~mL})$. The layers were separated, and the 
organic layer was washed with water $(2 \times 60 \mathrm{~mL})$ and sat. aqueous $\mathrm{NaCl}$ solution $(60 \mathrm{~mL})$, dried over $\mathrm{MgSO}_{4}$ and the solvents were evaporated in vacuo. The crude product was purified by column chromatography on silica gel using pentane/EtOAc 95:5. The desired product 3 (11.35 g, $24.8 \mathrm{mmol}, 85 \%)$ was obtained as clear oil.

${ }^{1} \mathrm{H}-\mathrm{NMR}\left(\mathrm{CDCl}_{3}, 300 \mathrm{MHz}\right): \delta=4.59(\mathrm{~d}, J=3.6 \mathrm{~Hz}, 1 \mathrm{H}, \mathrm{H}-1), 4.29(\mathrm{dd}, J=5.7,2.4 \mathrm{~Hz}$, $1 \mathrm{H}, \mathrm{H}-4), 4.19-4.08(\mathrm{~m}, 2 \mathrm{H}, 3-\mathrm{H}, \mathrm{H}-3), 3.74$ (dd, J = 7.2, 3.6 Hz, $1 \mathrm{H}, \mathrm{H}-2), 3.44$ (s, $\left.3 \mathrm{H}, \mathrm{OCH}_{3}\right), 3.41-3.29\left(\mathrm{~m}, 2 \mathrm{H}, \mathrm{H}-6, \mathrm{H}-6^{\prime}\right), 1.48\left(\mathrm{~s}, 3 \mathrm{H}, \mathrm{C}\left(\mathrm{CH}_{3}\right)_{2}\right), 1.34(\mathrm{~s}, 3 \mathrm{H}$, $\left.\mathrm{C}\left(\mathrm{CH}_{3}\right)_{2}\right), 0.98\left(\mathrm{~s}, 9 \mathrm{H}, \mathrm{SiC}\left(\mathrm{CH}_{3}\right)_{3}\right), 0.10\left(\mathrm{~s}, 3 \mathrm{H}, \mathrm{Si}\left(\mathrm{CH}_{3}\right)_{2}\right), 0.08\left(\mathrm{~s}, 3 \mathrm{H}, \mathrm{Si}\left(\mathrm{CH}_{3}\right)_{2}\right) \mathrm{ppm}$.

${ }^{13} \mathrm{C}$-NMR $\left(\mathrm{CDCl}_{3}, 126 \mathrm{MHz}\right): \delta=109.2\left(\mathrm{C}_{\left.\left(\mathrm{CH}_{3}\right)_{2}\right),}, 100.2(\mathrm{C}-1), 76.9(\mathrm{C}-3), 74.0(\mathrm{C}-4)\right.$, $71.2(\mathrm{C}-2), 68.6(\mathrm{C}-5), 55.8\left(\mathrm{OCH}_{3}\right), 28.1\left(\mathrm{C}\left(\mathrm{CH}_{3}\right)_{2}\right), 26.2\left(\mathrm{C}\left(\mathrm{CH}_{3}\right)_{2}\right), 25.8\left(\mathrm{SiC}\left(\mathrm{CH}_{3}\right)_{3}\right)$, $18.1\left(\mathrm{SiC}\left(\mathrm{CH}_{3}\right)_{3}\right), 2.85(\mathrm{C}-6), 4.58\left(\mathrm{Si}\left(\mathrm{CH}_{3}\right)_{2}\right), 4.75\left(\mathrm{Si}\left(\mathrm{CH}_{3}\right)_{2}\right) \mathrm{ppm}$.

MS (ESI): $\mathbf{m} / \mathbf{z}(\%)=481.1(100)[\mathrm{M}+\mathrm{Na}]^{+}$.

HRMS (ESI): calc. for $\mathrm{C}_{16} \mathrm{H}_{31} \mathrm{O}_{5} \mathrm{SiNa}[\mathrm{M}+\mathrm{Na}]^{+}: 481.0878$, found 481.0878 .

\section{(2R,3S,4S)-3,4-0-Isopropylidene-2-0-tertbutyldimethylsilyl-hex-5-en-1-al (4)}

Galactopyranoside 3 (32.6 g, $71.2 \mathrm{mmol}, 1.0$ eq.) and was dissolved in $\mathrm{THF} / \mathrm{H}_{2} \mathrm{O}$ (9:1, $\mathrm{v}, \mathrm{v}, 600 \mathrm{~mL}$ ) and zinc powder $(46.5 \mathrm{~g}, 710 \mathrm{mmol}, 10.0 \mathrm{eq}$.) was added. The suspension was stirred at $50^{\circ} \mathrm{C}$ for $6 \mathrm{~h}$. After cooling to ambient temperature, the suspension was filtered through a silica plug and washed with $\mathrm{Et}_{2} \mathrm{O}(300 \mathrm{~mL})$. The organic layer was washed with water $(200 \mathrm{~mL})$, sat. aqueous $\mathrm{NaHCO}_{3}$ solution $\left(200 \mathrm{~mL}\right.$ ) and sat. aqueous $\mathrm{NaCl}$ solution $(200 \mathrm{~mL})$, dried over $\mathrm{MgSO}_{4}$ and the solvents were evaporated in vacuo to give the desired product $4(21.2 \mathrm{~g}, 70.6 \mathrm{mmol}, 99 \%)$ as yellowish oil.

${ }^{1} \mathrm{H}-\mathrm{NMR}\left(\mathrm{CDCl}_{3}, 300 \mathrm{MHz}\right): \delta=9.67(\mathrm{~d}, J=0.9 \mathrm{~Hz}, 1 \mathrm{H}, \mathrm{CHO}$ ), 5.92 (ddd, $J=17.3$, 10.4, $6.8 \mathrm{~Hz}, 1 \mathrm{H}, H-5), 5.35$, (dt, $J=17.3,1.5 \mathrm{~Hz}, 1 \mathrm{H}, H-6), 5.21$ (dt, $J=10.4,1.5 \mathrm{~Hz}$, $\left.1 \mathrm{H}, H-6^{\prime}\right), 4.72(\mathrm{t}, J=6.8 \mathrm{~Hz}, 1 \mathrm{H}, \mathrm{H}-4), 4.32(\mathrm{dd}, J=6.8,5.3 \mathrm{~Hz}, 1 \mathrm{H}, \mathrm{H}-3), 4.07$ (dd, $J=5.3,0.9 \mathrm{~Hz}, 1 \mathrm{H}, \mathrm{H}-2), 1.51\left(\mathrm{~s}, 3 \mathrm{H}, \mathrm{C}\left(\mathrm{CH}_{3}\right)_{2}\right), 1.35\left(\mathrm{~s}, 1 \mathrm{H}, \mathrm{C}\left(\mathrm{CH}_{3}\right)_{2}\right), 0.92(\mathrm{~s}, 9 \mathrm{H}$, $\left.\mathrm{SiC}\left(\mathrm{CH}_{3}\right)_{3}\right), 0.09\left(\mathrm{~d}, J=1.8 \mathrm{~Hz}, 6 \mathrm{H}, \mathrm{Si}\left(\mathrm{CH}_{3}\right)_{2}\right) \mathrm{ppm}$.

${ }^{13} \mathrm{C}$-NMR ( $\left.\mathrm{CDCl}_{3}, 126 \mathrm{MHz}\right): \delta=201.8(\mathrm{CHO}), 133.8(\mathrm{C}-5), 118.9(\mathrm{C}-6), 109.2$ $\left.\left(\mathrm{C}\left(\mathrm{CH}_{3}\right)_{2}\right), 79.1(\mathrm{C}-3), 78.5(\mathrm{C}-4), 77.4(\mathrm{C}-2), 27.1\left(\mathrm{C}_{(\mathrm{CH}}\right)_{2}\right), 25.9\left(\mathrm{SiC}\left(\mathrm{CH}_{3}\right)_{3}\right), 25.2$ $\left(\mathrm{C}\left(\mathrm{CH}_{3}\right)_{2}\right), 18.4\left(\mathrm{SiC}\left(\mathrm{CH}_{3}\right)_{3}\right),-4.4\left(\mathrm{Si}\left(\mathrm{CH}_{3}\right)_{2}\right),-4.7\left(\mathrm{Si}\left(\mathrm{CH}_{3}\right)_{2}\right) \mathrm{ppm}$. 
MS (ESI): $\mathbf{m} / \mathbf{z}(\%)=323.2(0.5)[\mathrm{M}+\mathrm{Na}]^{+}$.

HRMS (ESI): calc. for $\mathrm{C}_{15} \mathrm{H}_{28} \mathrm{O}_{4} \mathrm{SiNa}[\mathrm{M}+\mathrm{Na}]^{+}: 323.1649$, found 323.1648.

\section{(2S,3S,4S)-3,4-0-Isopropylidene-2-O-tertbutyldimethylsilyl-hepta-1,5-diene (5)}

Under an argon atmosphere aldehyde 4 (21.2 g, $70.6 \mathrm{mmol}, 1.0 \mathrm{eq}$.$) was dissolved in$ dry THF $(200 \mathrm{~mL})$ and the solution was cooled to $0{ }^{\circ} \mathrm{C}$. TEBbe REAGENT $(0.5 \mathrm{M}$ in toluene, $200 \mathrm{~mL}, 100 \mathrm{mmol}, 1.4$ eq.) was added dropwise and the resulting solution was stirred at $0{ }^{\circ} \mathrm{C}$ for $30 \mathrm{~min}$. Subsequently $\mathrm{Et}_{2} \mathrm{O}(200 \mathrm{~mL})$ was added and water $\left(12 \mathrm{~mL}\right.$ ) was added dropwise. The resulting suspension was dried over $\mathrm{MgSO}_{4}$, filtered and the residue was washed with $\mathrm{Et}_{2} \mathrm{O}(100 \mathrm{~mL})$. The solvents of the combined organic layers were evaporated in vacuo and the resulting crude product was purified by column chromatography on silica gel using pentane/EtOAc 95:5. The desired product 5 (14.1 g, $47.1 \mathrm{mmol}, 67 \%)$ was obtained as an orange oil.

1'H-NMR $\left(\mathrm{CDCl}_{3}, 300 \mathrm{MHz}\right): \delta=6.04-5.80(\mathrm{~m}, 2 \mathrm{H}, \mathrm{H}-2, \mathrm{H}-6), 5.41-5.12(\mathrm{~m}, 4 \mathrm{H}, \mathrm{H}-1$, $\mathrm{H}-7$ ), 4.48-4.41 (m. $1 \mathrm{H}, \mathrm{H}-5), 4.22-4.14(\mathrm{~m}, 1 \mathrm{H}, \mathrm{H}-4), 4.02-3.95(\mathrm{~m}, 1 \mathrm{H}, \mathrm{H}-3), 1.50$ (s, $\left.3 \mathrm{H}, \mathrm{C}\left(\mathrm{CH}_{3}\right)_{2}\right), 1.35\left(\mathrm{C}\left(\mathrm{CH}_{3}\right)_{2}\right), 0.91\left(\mathrm{~s}, 9 \mathrm{H}, \mathrm{SiC}\left(\mathrm{CH}_{3}\right)_{3}\right), 0.09\left(\mathrm{~s}, 3 \mathrm{H}, \mathrm{Si}\left(\mathrm{CH}_{3}\right)_{2}\right), 0.07$ (s, $\left.3 \mathrm{H}, \mathrm{Si}\left(\mathrm{CH}_{3}\right)_{2}\right) \mathrm{ppm}$.

${ }^{13}$ C-NMR $\left(\mathrm{CDCl}_{3}, 126 \mathrm{MHz}\right): \delta=136.9$ (C-2), 134.8 (C-6), 118.7 (C-7), $116.6(C-1)$, $108.7\left(\mathrm{C}\left(\mathrm{CH}_{3}\right)_{2}\right), 82.0(\mathrm{C}-4), 79.2(\mathrm{C}-5), 72.7(\mathrm{C}-3), 28.0\left(\mathrm{C}\left(\mathrm{CH}_{3}\right)_{2}\right), 26.0\left(\mathrm{SiC}\left(\mathrm{CH}_{3}\right)_{3}\right)$, $25.7\left(\mathrm{C}\left(\mathrm{CH}_{3}\right)_{2}\right), 18.6\left(\mathrm{SiC}\left(\mathrm{CH}_{3}\right)_{3}\right),-4.4\left(\mathrm{Si}\left(\mathrm{CH}_{3}\right)_{2}\right),-4.5\left(\mathrm{Si}\left(\mathrm{CH}_{3}\right)_{2}\right) \mathrm{ppm}$.

MS (ESI): $\mathbf{m} / \mathbf{z}(\%)=321.2(14)[\mathrm{M}+\mathrm{Na}]^{+}$.

HRMS (ESI): calc. for $\mathrm{C}_{16} \mathrm{H}_{30} \mathrm{O}_{3} \mathrm{SiNa}[\mathrm{M}+\mathrm{Na}]^{+}:$321.1856, found 321.1854.

(2S,3S,4S)-2-Hydroxy-3,4-O-isopropylidene-hepta-1,5-diene (6)

Diene 5 (13.6 g, $45.6 \mathrm{mmol}, 1.0$ eq.) was dissolved in THF (500 mL) and a solution of TBAF $3 \mathrm{H}_{2} \mathrm{O}$ (28.8 g, $91.2 \mathrm{mmol}, 2.0$ eq.) in THF (100 mL) was added dropwise. The solution was stirred for $2.5 \mathrm{~h}$ at ambient temperature. The solvent was evaporated in vacuo and the resulting crude product was purified by column chromatography on silica gel using pentane/EtOAc 5:1. The desired product $6(8.12 \mathrm{~g}, 44.1 \mathrm{mmol}, 97 \%)$ was obtained as yellow oil. 
${ }^{1} \mathrm{H}-\mathrm{NMR}\left(\mathrm{CDCl}_{3}, 300 \mathrm{MHz}\right): \delta=6.07-5.93(\mathrm{~m}, 1 \mathrm{H}, \mathrm{H}-2), 5.90-5.77(\mathrm{~m}, 1 \mathrm{H}, \mathrm{H}-6), 5.44-$ 5.17 (m, $4 \mathrm{H}, \mathrm{H}-1, \mathrm{H}-7$ ), 4.63-4.55 (m, $1 \mathrm{H}, \mathrm{H5}$ ), 4.16-4.04 (m, $2 \mathrm{H}, \mathrm{H}-4, \mathrm{H}-3), 1.52$ (s, $\left.3 \mathrm{H}, \mathrm{C}\left(\mathrm{CH}_{3}\right)_{2}\right), 1.38\left(\mathrm{~s}, 3 \mathrm{H}, \mathrm{C}\left(\mathrm{CH}_{3}\right)_{2}\right) \mathrm{ppm}$.

${ }^{13} \mathrm{C}-\mathrm{NMR}\left(\mathrm{CDCl}_{3}, 126 \mathrm{MHz}\right): \delta=136.8(C-2), 134.1(\mathrm{C}-6), 119.5(C-7), 117.2(C-1)$,

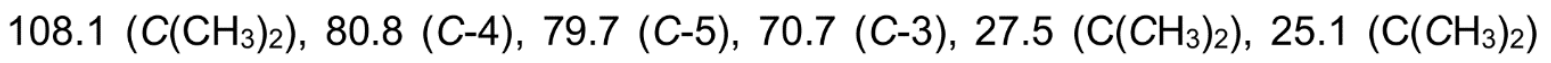
ppm.

MS (ESI): $\mathbf{m} / \mathbf{z}(\%)=207.1(7)[\mathrm{M}+\mathrm{Na}]^{+}$.

HRMS (ESI): calc. for $\mathrm{C}_{10} \mathrm{H}_{16} \mathrm{O}_{3} \mathrm{Na}[\mathrm{M}+\mathrm{Na}]^{+}: 207.0992$, found 207.0992 .

\section{(1S,2S,3S)-1-Hydroxy-2,3-0-isopropylidene-cyclopent-4-ene (7)}

Under an argon atmosphere diene 6 (8.12 g, $44.1 \mathrm{mmol}, 1.0 \mathrm{eq}$.$) and GRUBB's$ CATALYST, $2^{\text {ND }}$ GEN. (2.3 mol\%, $848 \mathrm{mg}, 1.0$ eq.) were dissolved in dry DCM $(200 \mathrm{~mL})$ and the solution was stirred under reflux for $1 \mathrm{~h}$. After cooling to ambient temperature, the solvent was evaporated in vacuo and the resulting crude product was purified by column chromatography on silica gel using pentane/EtOAc 2:1. The desired product 7 (5.2 g, $32.7 \mathrm{mmol}, 74 \%)$ was obtained as brown oil.

${ }^{1} \mathrm{H}-\mathrm{NMR}\left(\mathrm{CDCl}_{3}, 300 \mathrm{MHz}\right): \delta=6.00(\mathrm{~d}, J=5.7 \mathrm{~Hz}, 1 \mathrm{H}, H-5), 5.88(\mathrm{dd}, J=5.7,2.0 \mathrm{~Hz}$, $1 \mathrm{H}, H-4), 5.27$ (dd, J = 5.6, $1.0 \mathrm{~Hz}, 1 \mathrm{H}, H-3), 4.76$ (s, $1 \mathrm{H}, H-2), 4.49$ (d, J = $5.6 \mathrm{~Hz}$, $1 \mathrm{H}, \mathrm{H}-1), 1.38\left(\mathrm{~s}, 3 \mathrm{H}, \mathrm{C}\left(\mathrm{CH}_{3}\right)_{2}\right), 1.33\left(\mathrm{~s}, 3 \mathrm{H}, \mathrm{C}\left(\mathrm{CH}_{3}\right)_{2}\right) \mathrm{ppm}$.

${ }^{13} \mathrm{C}-\mathrm{NMR}\left(\mathrm{CDCl}_{3}, 126 \mathrm{MHz}\right): \delta=135.5(\mathrm{C}-5), 134.8(\mathrm{C}-4), 111.8\left(\mathrm{C}\left(\mathrm{CH}_{3}\right)_{2}\right), 86.0(\mathrm{C}-3)$, 84.4 (C-2), $81.1(\mathrm{C}-1), 27.4\left(\mathrm{C}\left(\mathrm{CH}_{3}\right)_{2}\right), 25.8\left(\mathrm{C}_{\left.\left(\mathrm{CH}_{3}\right)_{2}\right) \mathrm{ppm} .}\right.$

MS (ESI): $\mathbf{m} / \mathbf{z}(\%)=179.1(4)[\mathrm{M}+\mathrm{Na}]^{+}$.

HRMS (ESI): calc. for $\mathrm{C}_{8} \mathrm{H}_{11} \mathrm{O}_{2} \mathrm{BrNa}[\mathrm{M}+\mathrm{Na}]^{+}:$179.0679, found 179.0678 .

\section{(1R,2S,3S)-1-Bromo-2,3-0-isopropylidene-cyclopent-4-ene (8)}

Triphenylphosphine (16.1 g, $61.4 \mathrm{mmol}, 2.0$ eq.) was dissolved in DCM (300 mL) and cooled to $-10{ }^{\circ} \mathrm{C}$ and a solution of bromine $(3.1 \mathrm{~mL}, 61.4 \mathrm{mmol}, 2.0$ eq.) in DCM $(20 \mathrm{~mL})$ was added dropwise. The resulting solution was added slowly to a solution of alcohol 7 (4.8 g, 30.7 mmol, 1.0 eq.) and imidazole (2.3 g, $33.8 \mathrm{mmol}, 1.1$ eq.) in DCM 
$\left(150 \mathrm{~mL}\right.$ ) at $-10^{\circ} \mathrm{C}$. The reaction mixture was stirred at $-10{ }^{\circ} \mathrm{C}$ for $45 \mathrm{~min}$, then allowed to reach ambient temperature and $\mathrm{Et}_{2} \mathrm{O}(300 \mathrm{~mL})$ was added. The resulting organic solution was washed with water $(2 \times 300 \mathrm{~mL})$ and sat. aqueous $\mathrm{NaCl}$ solution $(300 \mathrm{~mL})$, dried over $\mathrm{MgSO}_{4}$ and the solvents were evaporated in vacuo. The residue was dissolved in DCM $(10 \mathrm{~mL})$ and hexane $(500 \mathrm{~mL})$ was added. The resulting suspension was filtrated, and the filtrate was concentrated in vacuo. The residue was suspended in hexane $(150 \mathrm{~mL})$ and filtrated. The filtrate was concentrated in vacuo and the resulting crude product purified by column chromatography on silica gel using pentane/EtOAc 95:5. The desired product 8 (5.1 g, $23.3 \mathrm{mmol}, 76 \%)$ was obtained as colorless oil.

${ }^{1} \mathrm{H}-\mathrm{NMR}\left(\mathrm{CDCl}_{3}, 300 \mathrm{MHz}\right): \delta=6.01-5.97(\mathrm{~m}, 1 \mathrm{H}, \mathrm{H}-5), 5.97-5.93(\mathrm{~m}, 1 \mathrm{H}, \mathrm{H}-4), 5.32$ (d, $J=5.4 \mathrm{~Hz}, 1 \mathrm{H}, H-3), 4.96(\mathrm{~d}, J=5.4 \mathrm{~Hz}, 1 \mathrm{H}, H-2), 4.86-4.82(\mathrm{~m}, 1 \mathrm{H}, H-1), 1.38$ (s, $\left.3 \mathrm{H}, \mathrm{C}\left(\mathrm{CH}_{3}\right)_{2}\right), 1.34$ (s, $\left.3 \mathrm{H}, \mathrm{C}\left(\mathrm{CH}_{3}\right)_{2}\right) \mathrm{ppm}$.

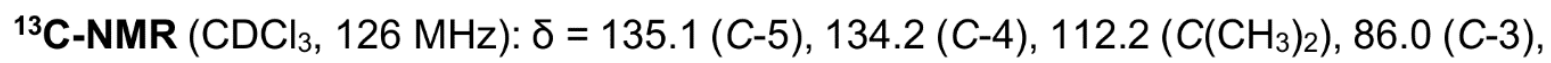

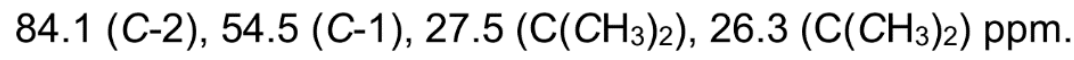

MS (ESI): $\mathbf{m} / \mathbf{z}(\%)=241.0(7)[\mathrm{M}+\mathrm{Na}]^{+}$.

HRMS (ESI): calc. for $\mathrm{C}_{8} \mathrm{H}_{11} \mathrm{O}_{2} \mathrm{BrNa}[\mathrm{M}+\mathrm{Na}]^{+}: 240.9835$, found 240.9833 .

\section{2',3'-O-Isopropylidene-queuine (10)}

Under an argon atmosphere pre-Q (9) $(1.0 \mathrm{~g}, 4.0 \mathrm{mmol}, 1.0 \mathrm{eq}$.) was dissolved in DMF (15 mL), DBU (6.0 mL, $40.0 \mathrm{mmol}, 10.0$ eq.) was added and the solution was stirred at $50^{\circ} \mathrm{C}$ for $15 \mathrm{~min}$. A solution of bromine $8(1.1 \mathrm{~g}, 4.8 \mathrm{mmol}, 1.2 \mathrm{eq}$.) in DMF $(3 \mathrm{~mL})$ was added dropwise and the solution was stirred at $50^{\circ} \mathrm{C}$ for $5 \mathrm{~h}$. After cooling to ambient temperature, the solvent was evaporated in vacuo and the residue was purified by column chromatography on silica gel using EtOAc/MeOH/NEt 3 3:1:0.1. The resulting crude product was suspended in $\mathrm{CHCl}_{3}(100 \mathrm{~mL})$ and stirred for $10 \mathrm{~min}$. The solid components were separated by filtration and suspended in methanol $(30 \mathrm{~mL})$. The suspension was filtrated, and the filtrate was concentrated in vacuo to yield the desired product 10 (581 mg, $1.64 \mathrm{mmol}, 41 \%$ ) as a slightly yellow solid.

${ }^{1} \mathrm{H}-\mathrm{NMR}\left(\mathrm{CD}_{3} \mathrm{OD}, 300 \mathrm{MHz}\right): \delta=6.90(\mathrm{~s}, 1 \mathrm{H}, \mathrm{H}-8), 6.30(\mathrm{dt}, J=5.9 \mathrm{~Hz}, 1.7 \mathrm{~Hz}, 1 \mathrm{H}$, $\left.H-5^{\prime}\right), 6.01\left(\mathrm{dm}, J=5.9 \mathrm{~Hz}, 1 \mathrm{H}, H-4^{\prime}\right), 5.36\left(\mathrm{dm}, J=5.8 \mathrm{~Hz}, 1 \mathrm{H}, H-3^{\prime}\right), 4.94(\mathrm{~d}$, 
$J=5.7 \mathrm{~Hz}, 1 \mathrm{H}, \mathrm{H}-2), 4.49-4.29\left(\mathrm{~m}, 3 \mathrm{H}, \mathrm{H}-1^{\prime}, \mathrm{H}_{2}-10\right), 1.37\left(\mathrm{~s}, 3 \mathrm{H}, \mathrm{C}\left(\mathrm{CH}_{3}\right)_{2}\right), 1.36$ (s, $\left.3 \mathrm{H}, \mathrm{C}\left(\mathrm{CH}_{3}\right)_{2}\right) \mathrm{ppm}$.

MS (ESI): $\mathbf{m} / \mathbf{z}(\%)=318.2(100)[\mathrm{M}+\mathrm{H}]^{+}, 340.2(4)[\mathrm{M}+\mathrm{Na}]^{+}$.

HRMS (ESI): calc. for $\mathrm{C}_{15} \mathrm{H}_{20} \mathrm{~N}_{5} \mathrm{O}_{3}[\mathrm{M}+\mathrm{H}]^{+}: 318.1561$, found 318.1562 .

\section{Queuine (11)}

2',3'-O-Isopropylidene-queuine (10) (581 mg, $1.64 \mathrm{mmol}, 1.0$ eq.) was dissolved in methanol $(60 \mathrm{~mL})$ and conc. $\mathrm{HCl}$ solution $(5 \mathrm{~mL})$ was added. The solution was stirred under reflux for $2 \mathrm{~h}$. After cooling to ambient temperature, the resulting suspension was concentrated in vacuo to yield queuine (11) (544 mg, $1.57 \mathrm{mmol}, 96 \%)$ as a slightly yellow solid.

${ }^{1} \mathrm{H}-\mathrm{NMR}\left(\mathrm{CD}_{3} \mathrm{OD}, 300 \mathrm{MHz}\right): \delta=7.04(\mathrm{~s}, 1 \mathrm{H}, \mathrm{H}-8), 6.28$ (dt, $\left.\mathrm{J}=6.3,2.2 \mathrm{~Hz}, 1 \mathrm{H}, H-5^{\prime}\right)$, 6.10 (dd, J = 6.3, 1.7 Hz, $\left.1 \mathrm{H}, H-4^{\prime}\right), 4.71-4.65$ (m, $\left.1 \mathrm{H}, H-3^{\prime}\right), 4.55-4.25$ (m, 4 H, H-2', $\left.H-1^{\prime}, H_{2}-10\right) \mathrm{ppm}$.

${ }^{13} \mathrm{C}-\mathrm{NMR}\left(\mathrm{CDCl}_{3}, 126 \mathrm{MHz}\right): \delta=161.8(C-6), 152.6(C-2), 146.6(C-4), 138.9\left(C-4{ }^{\prime}\right)$, 129.8 (C-5'), 121.0 (C-7), 109.8 (C-8), 99.9 (C-5), 74.7 (C-3'), $74.0\left(C-2\right.$ '), $87.8\left(C-1^{\prime}\right)$, $42.6(C-10) \mathrm{ppm}$.

MS (ESI): m/z (\%) = $278.1(95)[\mathrm{M}+\mathrm{H}]^{+}, 276.1(100)[\mathrm{M}-\mathrm{H}]^{-}, 312.1(75)[\mathrm{M}+\mathrm{Cl}]^{-}$.

HRMS (ESI): calc. for $\mathrm{C}_{12} \mathrm{H}_{15} \mathrm{~N}_{5} \mathrm{O}_{3} \mathrm{Na}[\mathrm{M}+\mathrm{Na}]^{+}:$300.1067, found 300.1063. 


\section{References}

1 Akimoto, H. et al. Queuine analogues. Their synthesis and inhibition of growth of mouse L5178Y cells in vitro. J Med Chem 29, 1749-1753 (1986).

2 Akimoto, H., Imamiya, E., Hitaka, T., Nomura, H. \& Nishimura, S. Synthesis of Queuine, the Base of Naturally-Occurring Hypermodified Nucleoside (Queuosine), and Its Analogs. Journal of the Chemical Society-Perkin Transactions 1, 1637-1644, doi:DOI 10.1039/p19880001637 (1988).

3 Klepper, F., Polborn, K. \& Carell, T. Robust synthesis and crystal-structure analysis of 7-cyano7-deazaguanine (PreQ(0) base) and 7-(aminomethyl)-7-deazaguanine (PreQ(1) base). Helvetica Chimica Acta 88, 2610-2616, doi:DOI 10.1002/hlca.200590201 (2005).

4 Barnett, C. J. \& Grubb, L. M. Total synthesis of Q Base (Queuine). Tetrahedron 56, 9221-9225, doi:Doi 10.1016/S0040-4020(00)00895-4 (2000).

5 Brooks, A. F., Garcia, G. A. \& Showalter, H. D. H. A short, concise synthesis of queuine. Tetrahedron Letters 51, 4163-4165, doi:10.1016/j.tetlet.2010.06.008 (2010).

6 Gerber, H. D. \& Klebe, G. Concise and efficient syntheses of preQ1 base, Q base, and (ent)-Q base. Org Biomol Chem 10, 8660-8668, doi:10.1039/c2ob26387d (2012).

7 Moynihan, L., Chadda, R., McArdle, P. \& Murphy, P. V. Allylic Azide Rearrangement in Tandem with Huisgen Cycloaddition for Stereoselective Annulation: Synthesis of C-Glycosyl Iminosugars. Organic Letters 17, 6226-6229, doi:10.1021/acs.orglett.5b03209 (2015).

8 Smith, A. B., Sperry, J. B. \& Han, Q. Syntheses of (-)-oleocanthal, a natural NSAID found in extra virgin olive oil, the (-)-deacetoxy-oleuropein aglycone, and related analogues. Journal of Organic Chemistry 72, 6891-6900, doi:10.1021/jo071146k (2007). 
a
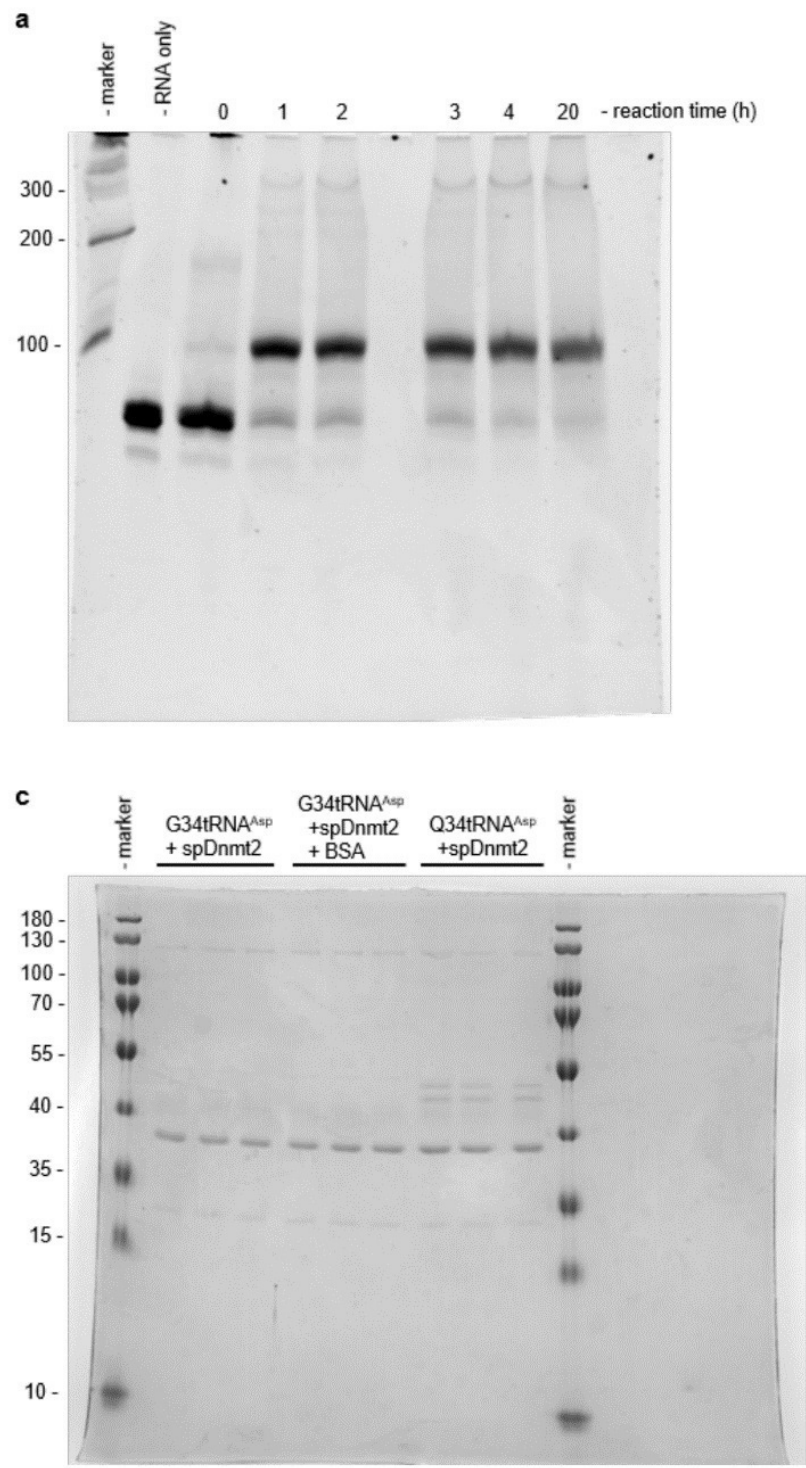

b

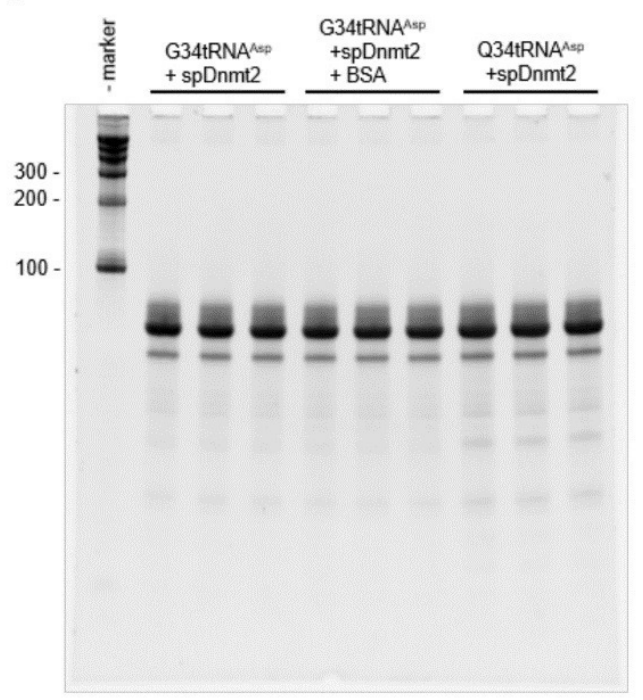

\section{Supplementary Figure S5. Uncropped gel images}

a Original image of the 3-phenylboronic acid containing denaturing $10 \%$ polyacrylamide gel shown in supplementary Figure S1. Ribo Ruler low range ladder (Fermantas) was used as a marker. Marker band-sizes are indicated as bases. b Original, image of the denaturing $10 \%$ polyacrylamide gel stained with GelRed (Biotinum) shown in supplementary Figure S2 part b. Ribo Ruler low range ladder (Fermantas) was used as a marker. Marker band-sizes are indicated as bases. c Original, image of the $12,5 \%$ SDS-polyacrylamide gel stained with Coomassie shown in supplementary Figure S2 part b. Prestained Protein Ladder (Fermentas) was used as a marker. Sizes of marker bands in kilodaltons are indicated on the left. The prominent band closely below the $40 \mathrm{kDa}$ marker band corresponds to spDnmt2. Samples containing Q34tRNA ${ }^{\text {Asp }}$ show an additional double band below $55 \mathrm{kDa}$ 
compared to G34tRNA Asp containing samples. These correspond to the two subunits of the TGT heterodimer that was used to enzymatically incorporate queuine into the tRNA. 


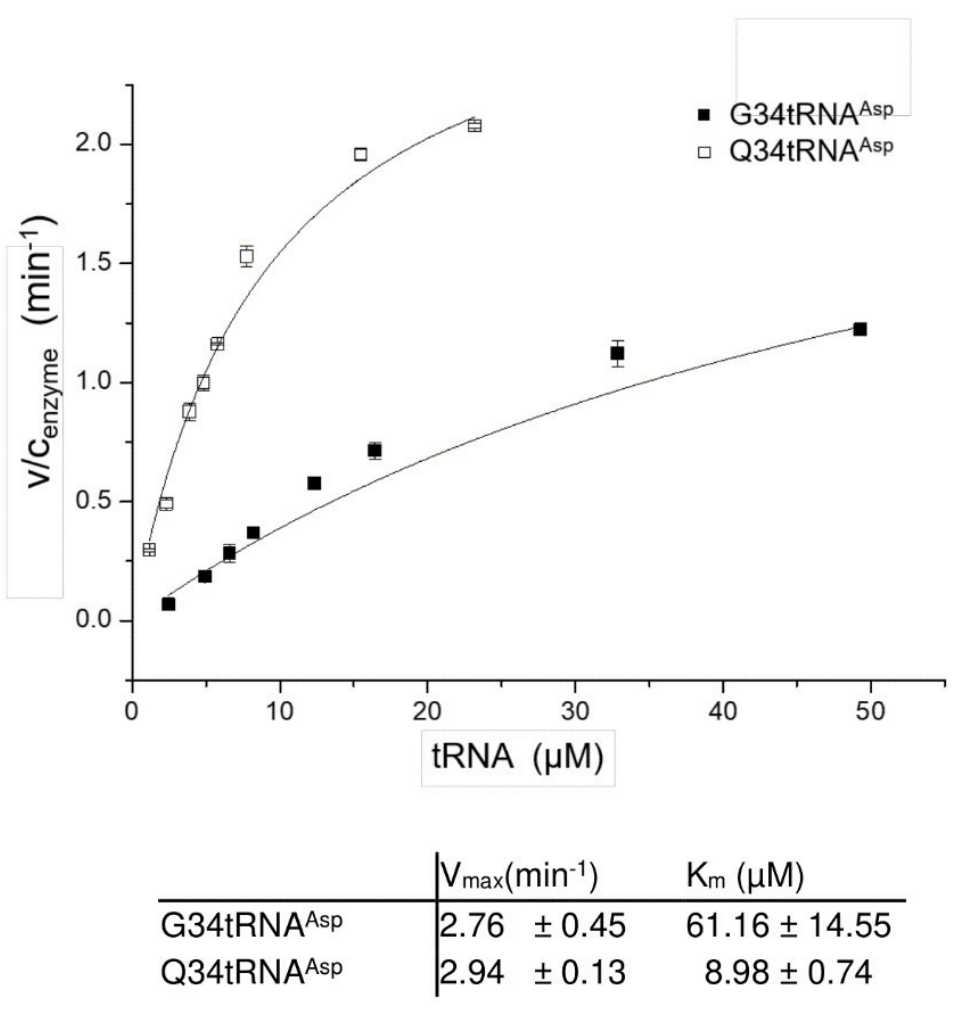

Supplementary Figure S6. Michealis-Menten fit of Dnmt2 Methyltransferase activity data using unmodified tRNA ${ }^{A s p}$ (G34tRNA ${ }^{\text {Asp }}$ ) and queuine harboring tRNA ${ }^{\text {Asp }}$ (Q34tRNA ${ }^{\text {Asp }}$ ) as substrates.

Activity is plotted as substrate conversion per enzyme concentration in $\min ^{-1}$. Measurements were performed as independent triplicates with increasing substrate concentrations. Errors are presented as standard deviation. Data points were fitted by employing the Hill equation with $\mathrm{n}$ fixed to 1 . 


\section{CHAPTER 4: DISCUSSION}

A complex cellular machinery is utilized by organisms for chemical modification of proteins/RNA after their translation/transcription to alter their chemical properties, extend their functionality, promote their structural integrity, or to fine tune cellular processes. In relation to the complex purpose of modifications, the chemical structure of these modifications is also highly diverse as are the "writers" and "readers" of these modifications. The importance of an interplay between modifications has been observed for modifications on histones, which have emerged to be highly interconnected and tightly regulated throughout the cell cycle to a complexity that this referred to as the "histone code" $(176,177)$. Such a coding of a complex interplay is not exclusive to proteins, but has also been proposed for certain glycosylations (178). The vast diversity of modifications would also provide the necessary framework for a similar interplay of modifications on RNA. However, for RNA the matter is much more diverse, due of the higher diversity of modifications and the existence of multiple RNAs. First evidence for an interplay of modifications on tRNA was reported after the start of this thesis showing that the presence of queuine modification does induce Dnmt 2 mediated methylation of the C38 base on the same tRNA molecule in vivo. The hallmark of this thesis is the structural and biochemical investigation of the two enzymes that deposit these two modifications in the eukaryotic system: The heterodimeric TGT and the methyltransferase Dnmt2.

Within this thesis, the first structure of a eukaryotic TGT catalytic subunit has been described confirming a conserved catalytic mechanism for the QTRT1 subunit from bacteria to mankind. While the publication focused of the active site, in this chapter the conservation of tRNA recognition by TGTs is further investigated and the effects of posttranslational phosphorylation will be discussed in context of the newly described QTRT1 structure.

Besides the structural investigation of the human TGT enzyme, the most extensively studied topic of this thesis is the biochemical and structural study of the influence of Q34 modification on Dnmt2 activity. As shown in chapter 3, queuine alone is sufficient to trigger Dnmt2 activity in vitro. To provide insights into how activity is modulated by queuine, a model of the enzyme substrate complex was computed, based on the newly reported S. pombe Dnmt 2 structure. Here, the model is compared to previously reported Dnmt2 substrate models. Furthermore, the crosslink data of the enzyme substrate complex offers the opportunity to evaluate the model complex 
with respect to information from in vitro complex formation. Moreover, different possibilities how queuine might influence Dnmt 2 on a molecular level will be evaluated and finally, a model for the Dnmt2 enzymatic turnover reaction will be proposed by linking previously reported data to the results of this thesis.

\subsection{INVESTIGATION OF RNA-INTERACTING RESIDUES IN QTRT1}

In the publication entitled "Crystal structure of the human TGT catalytic subunit QTRT1" (chapter 2) the general fold of the newly reported human QTRT1 crystal structure is compared to the crystal structure of the bacTGT identifying the fold of both proteins to be highly similar. Furthermore, the structural comparison of both active sites finds the catalytic residues of QTRT1 and the bacTGT at equivalent locations. This argues for a conserved reaction mechanism, despite of the different specificity for the incorporated modified base. However, the overall similar fold of both enzymes does not only allow to compare their active centers but also to evaluate the conservation of their RNA interactions. The base for this analysis is the QTRT1 structure and the complex structure of the Z. mobilis TGT enzyme which is covalently linked to an anticodon RNA substrate (PDB-ID: 1Q2R).

In the structure of the RNA in the complex with bacTGT the bases of the anticodon stem are paired, limiting their accessibility. Thus, protein RNA interactions involving this RNA region are restricted to contact formation of protein residues with the phosphate ribose backbone. In contrast, the unpaired bases of the stem loop are exposed and can interact with protein residues (Figure 12). The first base that is stacked, but not engaged in base pairing is the base 32 which is coordinated face to face by Gln290 in the complex structure. Interestingly, in the human QTRT1 enzyme the equivalent position is held by a phenylalanine, that could promote RNA interaction through $\pi$-stacking. Similarly, U33 also is coordinated by only one bacTGT residue, Asp267, which is conserved in human QTRT1. Coordination of the target ribose at position 34 is mediated mainly by the catalytic Asp280. Residues forming the catalytic site are conserved from the bacterial to the human enzyme, as it is described in chapter 2, arguing for an overall similar interaction at this base in both proteins. A base that exhibits extensive interaction with the protein is U35. This uridine is accommodated inside a grove on the bacTGT surface which is formed by two arginines. Coordination of U35 by this two residues involves both oxygens of the purine base, and thus likely is base specific. This specificity is conserved to human where 
a uridine is present at position 35 in all four tRNA substrates and the two arginine residues on the protein side (Arg285 and Arg288), highlighting the functional conservation of TGT enzymes. Another conserved residue is the Ser113 (Ser116 in human QTRT1) that coordinates A36 in the bacterial substrate complex. Despite the serine residues in both enzymes do occupy an equivalent position, the underlying polypeptide chains adopt different conformations. However, this difference might be less severe when QTRT1 is bound to a substrate tRNA, as this part of the protein chain exhibits high B-factors. This indicates flexibility in solution and furthermore the two beta strands comprised by the amino acids Gly124 to 138 adopt a more open conformation compared to the equivalent residues in the bacTGT complex. In the QTRT1 crystal, this conformation is stabilized through crystal contact formation and might be different when the tRNA substrate is bound. The RNA bases 37 and 38 in the bacTGT complex only show limited interactions with the protein despite they are not engaged in base pairing. In total, the comparison of the residues involved in contact with the RNA bases highlights a high conservation of these amino acids from the bacterial to human QTRT1. In contrast, the noncatalytic QTRT2 subunit of the eukaryotic enzyme does not exhibit this conservation with the respective residues exchanged by chemically different amino acids. Beside the incapability for base exchange, which has been discussed in chapter 2, this structural investigation agues for an impaired or even absent tRNA binding ability in QTRT2. 


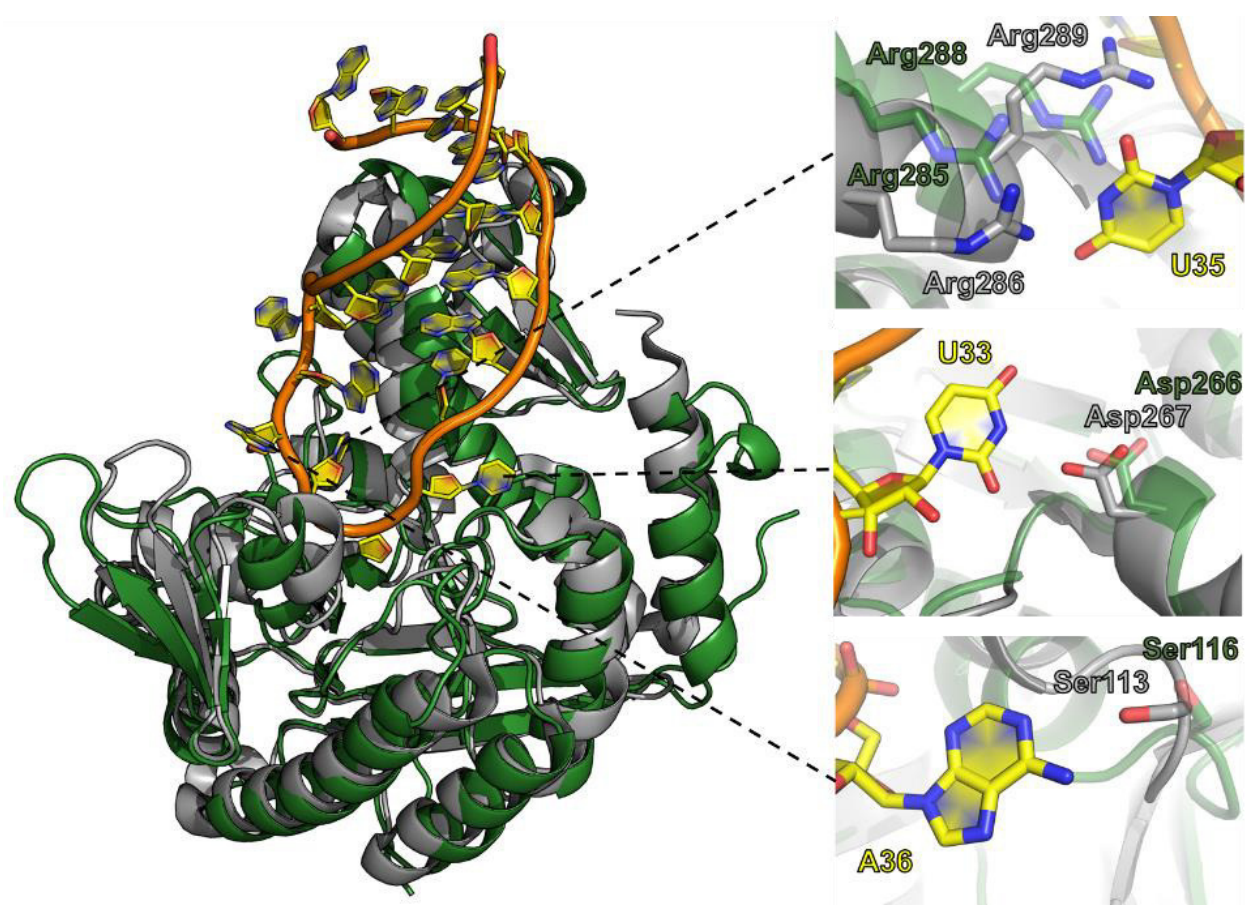

Figure 12. Conservation of TGT residues interacting with RNA.

(Left) overlay of the Z. mobilis bacTGT (grey) with the QTRT1 subunit structure (PDB-ID: 6H45) (green). The bacTGT was crystallized in complex with RNA (yellow/orange) (PDB-ID: 1Q2R). QTRT1 was aligned to the structure of the bacTGT monomer which is bound to the RNA, the other subunit is omitted from the figure. Unpaired RNA bases interact with several residues of the bacTGT. The vast majority of these residues is conserved to human QTRT1 (right).

\subsection{STRUCTURAL INVESTIGATION OF QTRT1 PHOSPHORYLATION}

Preparation of the human TGT from endogenous sources yields an active enzyme but its activity is lost over days (159). Interestingly, the activity can be restored by kinase activity, suggesting an involvement of posttranscriptional phosphorylation. Consequently, application of a phosphorylase results in a substantial decrease of TGT activity, supporting the hypothesis that indeed TGT phosphorylation regulates the enzymes activity (159). However, the underlying study fails to report the modification site resulting from PKC activity. Investigation, of reported phosphorylation for both subunits, QTRT1 and QTRT2, reveals six sites to carry phosphate modification while only one site was reported for QTRT2. However, most of these sites have been found in high throughput analysis and were automatically annotated without manual confirmation rendering it hard to confirm the validity of these results. A valuable exception is the phosphorylation of the QTRT1 Ser139 residue, which has also been confirmed in low 
throughput analysis (179). Investigation of this residue in context of the newly reported QTRT1 structure, finds Ser139 on the protein surface and exposed to the solvent and accessible for potential phosphorylation by PKC (Figure 13).

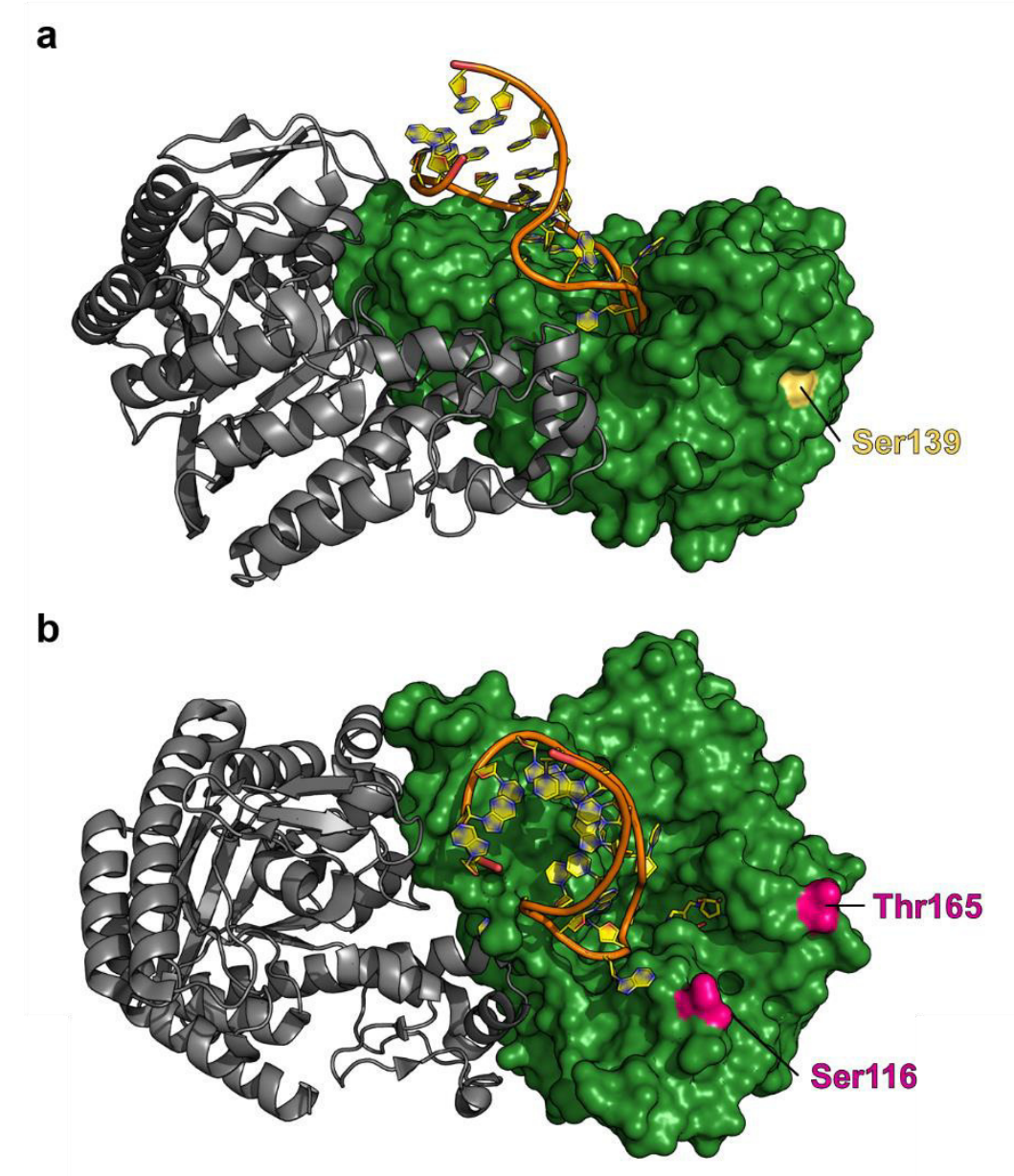

Figure 13. Analysis of QTRT1 phosphorylation.

a Overlay of the human QTRT1 monomer with the Z. mobilis homodimer crystallized in complex with RNA (PDB-ID: 1Q2R). The QTRT1 structure, shown as green surface representation, (PDB-ID: 6H45) was aligned to one subunit of the bacTGT dimer. This subunit is omitted from the image. The respective other subunit of the bacTGT homodimer is shown as grey cartoon to illustrate a potential dimerization of the human TGT. Ser139 (gold) is distant from a potential dimerization surface and the RNA (orange/yellow). Furthermore, Ser139 is also distant from the active site. b Top down view into the assembly shown in a. Ser116 (pink) is close to the RNA and Thr165 (pink) is in close proximity to the queuine base (yellow sticks).

However, Ser139 is quite distant from the active site. The distance of $15.1 \AA$ between the hydroxy group of the serine side chain and the nearest atom of the queuine base renders an influence of this peripheral residue on the architecture of the catalytic pocket questionable. Furthermore, based on an overlay of the QTRT1 structure with the structure of the bacTGT 
complex (PDB-ID: 1Q2R), interaction with a tRNA substrate is unlikely, assuming a similar tRNA binding to the human TGT. However, the Ser139 is directly C-terminal to the flexible three-stranded beta sheet, which is described in paragraph 2.4. A phosphorylation at this site could foster a conformation of the sheet and the interconnecting loops that aids tRNA binding. As a third possibility, Ser139 phosphorylation could modify the affinity of the heterodimer's subunits. Assuming a conservation of the dimer assembly, which has been observed to arrange similarly in bacTGT and QTRT2 homodimers, for the functional heterodimer, Ser139 is likely incapable to influence the dimerization as it is neither part of the interaction surface nor secondary structures involved in the contact formation between the two subunits (Figure 13a). In total, phosphorylation of Ser139 may be unlikely to promote human TGT activity. Furthermore, prediction of QTRT1 phosphorylation prediction with NetPhos 3.1 (180) finds a low probability for Ser139 phosphorylation by PKC. Instead, this residue is suggested to be a target for several other kinases, which have not been implicated into modulation of TGT activity. However, other residues of QTRT1 are suggested by the prediction to be targeted by PKC in the QTRT1 enzyme but have yet not been reported to undergo phosphorylation. Considering the impact of PKC dependent phosphorylation on TGT activity, candidate residues of the prediction pool may be close to the active site, RNA interacting regions or at the heterodimer interface, latter of which are still awaiting structural confirmation. However, no predicted phosphorylation site is located at the interface of both subunits, in case dimer architecture is conserved from bacteria to the eukaryotic TGT. Instead, Thr165, one of the putative PKC phosphorylation sites, is in close proximity to the queuine binding grove and to the hydroxy groups of cyclopentene-diol moiety (5.8 A distance between the sidechain and the C5 hydroxy group). A phosphorylation of this residue may promote binding of the queuine base through further coordination of the cyclopentene-diol upon a small rearrangement of the underlying peptide chain. This coordination could result in higher affinity and/or an optimized positioning of the queuine base for the nucleophilic attack on the reaction intermediate, thus increasing TGT activity. Interestingly, also the conserved Ser116 is predicted to be targeted by PKC. The corresponding serine in the RNA complex structure of the Z. mobilis bacTGT interacts with the adenine base at position 36 (Figure 12). Considering that this base is not conserved in the substrate tRNAs of human TGT, phosphorylation of this residue might promote contact formation with the less spacious pyrimidine bases of tRNA ${ }^{\text {Asp }}$ and RRNA $^{\text {Asn }}$. At this point it is unclear whether these depicted consequences of a Ser116 phosphorylation hold true, as Morris et al., confirmed the PKC dependent stimulation of TGT activity in context of total tRNA extract but not for the individual substrates (159). 
In total, it is undoubted that PKC mediated phosphorylation has a stimulating effect on the activity of the human TGT enzyme, however the phosphorylation of Ser139 reported for QTRT1 seems unlikely to have an impact on TGT activity. Instead, other sites on the catalytic subunit have been predicted to be targeted by PKC, with Thr165 and Ser116 being the best candidates whose phosphorylation might impact TGT activity. Anyhow, which residue indeed is targeted by PKC awaits conformation by subsequent TGT phosphorylation and its analysis by mass spectrometry.

\section{$\underline{\text { 4.3 DNMT2 SUBSTRATE SPECIFICITY }}$}

The methyltransferase Dnmt2 is the most conserved of all Dnmt enzymes (75), with Dnmt2 activity reported for several organism ranging from human, over fruit fly to yeast $(64,67,181)$. These studies mainly focus on the Dnmt 2 dependent methylation of the $\mathrm{C} 38$ base, the common target site for Dnmt2 mediated methyltransfer in all organisms that have reported Dnmt2 activity. The vast majority of published data on Dnmt 2 tRNA targets do include the accepted amino acid by the tRNA and may report the anticodon sequence, however, annotation of the underlying tRNA sequence or the genome locus is the exception. For identification of potential Dnmt 2 targets the missing link between the name of the reported target and its sequence is a general hinderance. The problem of insufficient annotation becomes apparent when a specific tRNA encoded in the human genome is of interest, as a total of 417 tRNA genes are mapped but only 61 codons code for amino acids. Moreover, different loci that code for a tRNA with a common anticodon do not necessarily have the same sequence. For example, nine genes for tRNA $^{\mathrm{Val}}$ with the anticodon $\mathrm{AAC}$ are annotated in the human genome, with five of them coding for the same sequence (tRNA ${ }^{\mathrm{Val}}-\mathrm{AAC}-1-1$ to $\left.\mathrm{tRNA}^{\mathrm{Val}}-\mathrm{AAC}-1-5\right)$. In this case a different tRNA sequence has been identified so serve as a substrate for Dnmt2, which is tRNA ${ }^{\mathrm{Val}}$-AAC-2-1 (81), highlighting the importance of sequence annotation for Dnmt2 substrates.

The report of queuine being important for Dnmt2 catalytic activity in $S$. pombe, points out the importance of the base position 34 for Dnmt 2 substrate tRNAs $(65,182)$. Whether queuine has a similar effect in the human system is currently unknown, but that C38 methylation of tRNA Asp by Dnmt2 in human is exclusively observed in concert with queuine modification of the wobble base (64) supports the idea that this position is of eminent importance for Dnmt2 activity. 
Interestingly, the importance of the wobble base for Dnmt2 activity seems not to be restricted to the tRNA $^{\text {Asp }}$ substrate. The sequence of the non cognate tRNA ${ }^{\mathrm{Val}}$ substrate in human (tRNA $^{\mathrm{Val}}$-CAC-2-1) does not code for a guanine at position 34 but for the pyrimidine base, cytosine. Interestingly, Khoddami et al. (81) observed no Dnmt2 methylation for tRNA ${ }^{\text {Val }}$ CAC-2-1 despite the almost identical sequence of both tRNAs. Notably, the only difference in the sequence of the two tRNAs is the respective exchange of the adenine at position 34 by the pyrimidine base cytosine, transforming a Dnmt2 substrate into a non-cognate tRNA. The apparent necessity for a purine base at position 34 for methyltransfer by the human enzyme is further supported by the third annotated tRNA substrate which is tRNA ${ }^{\text {Gly }}$ (tRNA ${ }^{\text {Gly }}$-GCC-11). Here, in analogy to tRNA ${ }^{\text {Asp }}$, position 34 is occupied by the purine base guanine. This finding, although it awaits further confirmation by biochemical studies, may serve as a hint that Dnmt2 indeed does distinguish its substrates by the anticodon sequence.

\subsection{IMPACT OF THE S. POMBE DNMT2 CRYSTAL STRUCTURE}

At the start of this thesis, three structures of Dnmt2 from human, S. frugiperda, and the pathogenic amoebae E. histolytica were available from X-ray crystallography (70, 72, 73). These structures offer insights into the enzyme's fold and accommodation of the methyldonor SAM. However, they provide only limited information about substrate binding as the structures do not contain a nucleic acid. Analysis of the three annotated tRNA substrates from the human Dnmt2 enzyme reveals high variances in the nucleotide sequence but their predicted secondary structures are highly similar. This structural similarity is also observed for the tRNA substrates which exhibit a high consensus of all the clover leaf secondary structures especially in the anticodon stem loop. In all three human tRNA targets the stem is comprised of five Watson-Crick base pairs, which in total have a generally high CG content, from $60 \%$ in tRNA $^{\mathrm{Val}}$ to $100 \%$ in $\mathrm{tRNA}^{\mathrm{Asp}}$, and an anticodon loop that comprises seven unpaired bases. Furthermore, these properties of the stem and the loop do also apply for the Dnmt 2 targets in D. melanogaster and S. pombe (11).

The structure of the $S$. pombe Dnmt2 (PDB-ID: 6FDF), which is reported as part of this thesis (182), exhibits a positively charged area around the negatively charged active site cavity. This cavity has to accommodate the $\mathrm{C} 38$ base in order to generate the close proximity of the substrates and catalytic residues necessary for the methyltransfer reaction. This charge 
distribution of the catalytic center and the surrounding area is not only a feature in the structure from $S$. pombe but also conserved in the other reported Dnmt2 structures. This evolutionary conservation indicates a defined purpose of this region in interaction with the tRNA. Considering the conserved positive charge and the above described seemingly conserved topology of the tRNA, interaction around the active site likely is mediated by the phosphate ribose backbone of the structurally similar tRNAs. Indeed, the positive charge is of importance for Dnmt2 activity as mutagenesis of non-catalytical residues involved in this charge topology results in decreased activity of the human Dnmt2 enzyme (69), highlighting the importance of this charge distribution. In conclusion, the conservation of surface electrostatics in regions adjacent to the active site supports the hypothesis that the protein substrate interaction is mainly facilitated by contact formation between the positively charged protein surface and the negatively charged phosphate backbone of the tRNA. This could also explain the seemingly conserved architecture, but not conserved sequence of the different tRNA substrates.

\section{$\underline{4.5 \text { BiologiCAL SigNIFICANCE OF DNMT2 COMPLEX MODELS }}$}

The molecular mechanism underlying the methyl transfer reaction catalyzed by Dnmt2 enzymes has been described by employing biochemical methods and found to resemble the mechanism used by other Dnmt family members (68). Beside this discovery, several efforts have been made to understand the modus of Dnmt2 substrate recognition providing valuable information about accepted substrates (71) and interacting regions of the protein surface (69). In total, two models of Dnmt 2 in a substrate complex have been proposed simultaneously before the start of this thesis. One reported model presents the Dnmt2 structure from E. histolytica in complex with a DNA double helix comprising a cytosine flipped out into the catalytic pocket of Dnmt2 (70). This model was generated by superposition of the Dnmt2 structure with the structure of the similar but unrelated DNA methyltransferase HhaI, which was solved in complex with a DNA double helix (PDB-ID: 1MHT). The model seems to fulfill the reported properties that are required for Dnmt 2 mediated methyltransfer. Furthermore, major clashes between both structures of the model do appear to be absent, which is aided by the structural similarity of both enzymes. This overlay represents a plausible model for DNA methylation by Dnmt2, but is still controversially discussed (59). As a consequence, the question of a biological significance of this model is yet unanswered. 
Nevertheless, Dnmt2 has indeed been shown to be capable of DNA methylation but only in the structural context of tRNA (71). In opposition to the discussion of DNA methylation, methylation of tRNA by Dnmt 2 has been observed by various groups $(64-66,81,183)$, leaving Dnmt2 activity on tRNA unquestionable. The second model of a Dnmt2 substrate complex was reported in frame of mapping the substrate binding site of the human Dnmt2 enzyme by a mutational study (69). This model comprises the structure of Dnmt2 as well as a tRNA Asp structure taken from an aspartyl-tRNA synthase complex (PDB-ID: 1ASY). The tRNA structure was manually placed into the Dnmt2 structure with respect to the surface residues which were found to be of importance for Dnmt2 activity (69). However, this model has certain flaws as monitoring of enzyme activity upon alteration of non-catalytic residues is not a direct measure for enzyme substrate interaction. Furthermore, the top down representation of the complex model, clearly limits the accessibility of details such as the distances between the two molecules. This representation may be sufficient to support the authors' conclusion that the tRNA stem and anticodon loop may be of suitable size to be accommodated by the groove around the active site, however the lack of details caused by the manual placing prevents further interpretation.

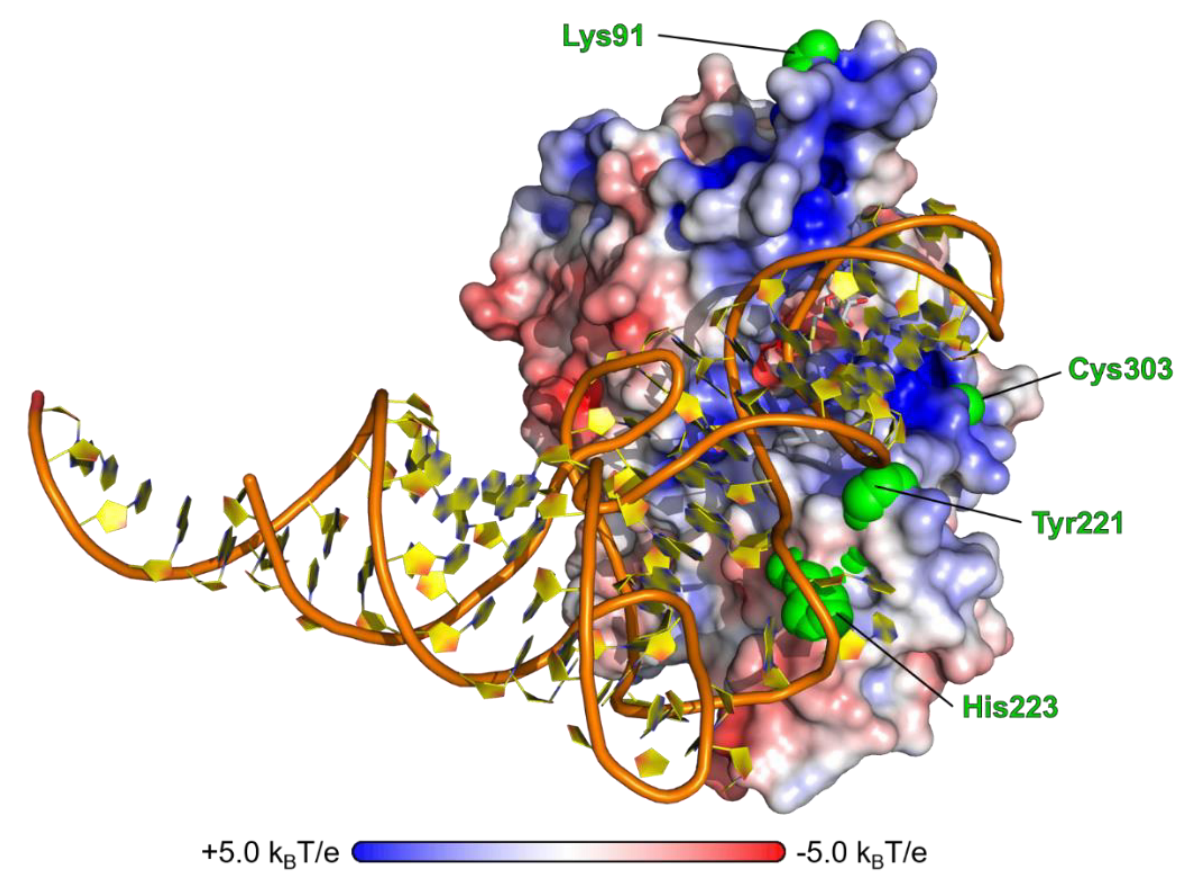

Figure 14. Validation of the Dnmt2 tRNA docking model.

The structure of tRNA ${ }^{\text {Asp }}$ from $S$. cerevisiae (PDB-ID: 1VTQ) that was docked to the $S$. pombe Dnmt2 crystal structure (PDB-ID: 6FDF) is depicted as cartoon. Dnmt2 is shown as surface representation with electrostatics ranging from $+5.0 \mathrm{k}_{\mathrm{B}} \mathrm{T} / \mathrm{e}$ (blue) to $-5.0 \mathrm{k}_{\mathrm{B}} \mathrm{T} / \mathrm{e}$ (red). Protein residues cross-linked to RNA bases are depicted as green spheres. 
The model of the tRNA ${ }^{\text {Asp }}$ Dnmt2 complex (Figure 14), which has been published under the title "Structural insights into the stimulation of $S$. pombe Dnmt2 catalytic efficiency by the tRNA nucleoside queuosine" and is reported in this thesis, is not based on manual placement or superposition. Instead, computational docking of five different tRNA structures (PDB-IDs: 1VTQ, 1ASZ, 1QF6, 4WJ3, 4WT8) was performed. Compared to manual placement, this method has the advantage of an automated quantitative ranking of the docked models through their individual internal Rosetta scores, a value that has been adjusted from normal Rosetta scores to better meet the properties protein RNA complexes (184). Application of this method prevents bias compared to manual placements of RNAs. However, selection of the best model was not solely based on a total score containing the energy of the interactions across the surface and various other values, but also on biochemical requirements. In this respect we verified the model with respect to the proximity of the methylation target $\mathrm{C} 38$ to the active site. Further justification of the model is achieved by mapping the interaction surface through cross-linking of the complex in solution that does allow the direct observation of protein-RNA interactions.

Several tRNA structures were used for docking but no confirmed Dnmt2 substrate structure was available in the Protein Data Bank. Interestingly, the best model was observed by docking the tRNA ${ }^{\text {Asp }}$ structure from S. cerevisiae (PDB-ID:1VTQ), one of the few organisms that do not encode Dnmt2 in their genome (56). This raises the question whether this model allows for a more detailed interpretation since the cross-linking was performed with tRNA and Dnmt2 both cloned from $S$. pombe sequences. Therefore, the sequences of the docked $S$. cerevisiae tRNA $^{\text {Asp }}$ and the $S$. pombe tRNA ${ }^{\text {Asp }}$ were analyzed. Both tRNA ${ }^{\text {Asp }}$ molecules are composed of 73 nucleotides, which do strongly differ in their sequence although they share the same anticodon sequence. A structural alignment of both sequences identified both tRNAs not only to encompass the same number of nucleotides but also the stems and loops are of identical sizes (185) arguing for an overall similar fold of the two tRNAs.

UV-light was used to cross-link the tRNA with the protein in the ternary complex and four amino acids were identified by terms of mass spectrometry that are in close contact with the tRNA molecule (Lys91, Trp221, His223 and Cys303). All crosslinks were observed exclusively to uridines. The closest cross-link to the active site involved the Cys303 residue. Evaluation of the proximity of the respective cysteine to the tRNA identifies the wobble base adjacent base 33 to be the closest and thus most likely to be cross-linked to Cys303 by UV-light. The corresponding base at position 33 in the $S$. pombe $\mathrm{tRNA}^{\mathrm{Asp}}$ indeed is a uridine (U33), finding the model in agreement with the biochemical data. During refinement of the structure, the 
unusual location of the tryptophan residue Trp221 was noticed as it is not buried in the protein core but exposed to the solvent. This is a rather unusual case for a hydrophobic sidechain indicating that this residue could be involved in tRNA recognition, presumably through $\pi$ stacking. Indeed, Trp221 as well as His223 apparently are in close distance to the tRNA in the ternary complex as they were found to cross-link to uridines. The docked model finds Trp221 in close contact with the tRNA bases 41 and 42, both of which are uridines in $S$. pombe tRNA ${ }^{\text {Asp }}$. On a side note, this case also nicely illustrates the limitations of the employed cross-link method, which involves the digest of the RNA during sample preparation for MS analysis. A consequence of this method is the loss of sequence information on the RNA side which makes it impossible to distinguish which one of the two uridines is involved in the cross-link. Closest to the cross-linked His223 residue are the bases 19 and 20 in the complex model, which are also the first two bases of the D-loop. In the tRNA structure, they are flipped out from the loop and facing the protein surface. Although base 20 is slightly closer to His223, only base 19 is a $\mathrm{U}$ in S. pombe tRNA ${ }^{\text {Asp }}$ and can be involved in the cross-link formation in the ternary complex. It seems that orientation of the tRNA in the model compared to the in-solution complex differs in regions that are remote to the active site. The fourth interaction between the protein and the RNA is observed for the active site loop harbored Lys91 which is rather distant from the tRNA, thus resulting in various interaction possibilities. Consequently, it is impossible to determine from the available data whether this residue interacts with U33, U35, or the even more distant nucleotides U41 or U42.

In summary, the information derived from cross-linking agrees very well with the proposed docking model of the Dnmt2 tRNA ${ }^{\text {Asp }}$ complex. However, in case of the cross-linked Lys91 our model also highlights the limitation that arise from docking of rigid body structures when it comes to flexible regions and altered conformations upon substrate binding. In this case, the conformation of the active site loop, which is observed in the crystal structure, is likely different when a substrate is bound. Interestingly, the conformation of this loop does not interfere with tRNA binding based on the docking model, arguing that it represents an "open" conformation. In case of tRNA docking the missing flexibility is of high importance as structures of tRNA complexes have been shown that the conformation of RNA molecules can undergo substantial changes when they are bound by a protein $(26,27,143)$. Usually, the most striking differences to the unbound tRNA molecule are observed in the interacting regions, which, in the case of Dnmt2, seems to involve the anticodon stem loop, the anticodon stem, and the D-loop. Consequently, these regions might adopt a different fold when the tRNA is bound by Dnmt2. 
Altogether, the application of unbiased computational docking methods, biological requirements and the evaluation of the models based on direct interactions between the tRNA and Dnmt 2 make the herein reported arrangement the most plausible model for a Dnmt 2 tRNA substrate complex reported to date.

\subsection{How Queuosine Might TRIGGER DNMT2 ACTIVITY}

Previously, Müller et al. (65) showed that methylation of tRNA Asp by Dnmt2 is dependent on presence of the nutrient queuine, which gets incorporated into position 34 of the tRNA. We could confirm that queuine modification of tRNA ${ }^{\text {Asp }}$ alone is sufficient to trigger Dnmt 2 activity in vitro and found that its presence on the substrate does lower the $\mathrm{K}_{0.5}$ value and increases $\mathrm{V}_{\max }$ in comparison to the unmodified substrate (182). However, the incorporation of queuine does only have a minor effect on binding affinity raising the question how queuosine affects the methyltransfer reaction. Co-crystallization of $S$. pombe Dnmt2 in presence of a micromolar concentration of the free queuine base, as well as soaking did not result in additional electron density that could correspond to this ligand. This finding together with the observation that queuine has only a mild effect on substrate affinity indicates that interaction of the queuosine base with the protein might be limited. At the same time, it also raises the question how queuine can have this tremendous effect on Dnmt 2 activity if it is not directly recognized by the protein. Its direct participation in the chemical reaction is also unlikely because the unmodified tRNA substrate is also methylated, however at lower turnover numbers. Furthermore, our model pictures queuosine in proximity of the active center but still too distant for a direct involvement in the reaction.

Queuosine might also have other effects in context of tRNA as modifications of the anticodon at position 34 and 37 have been implicated in tRNA conformation $(34,44)$. Initially these modifications were proposed to increase flexibility of the anticodon loop, maybe because they add more chemical groups to the loop chemistry. However, the opinion has emerged that they in fact promote rigidity of the tRNA anticodon loop (43). It is possible that Q34 may have such an effect on tRNA ${ }^{\text {Asp }}$ through stabilizing a conformation that weakens $\mathrm{C} 38$ interaction within the tRNA and promote the base flip.

As a third hypothesis Q34 could promote Dnmt2 activity through interaction with the active site loop. It is possible that the flexibility of this loop is altered by coordination of residues or 
the peptide backbone via the diol moiety of the queuine's cyclopentene ring or by stacking with the double bond. We tried to localize the queuosine protein interaction by cross-linking of the Q34 modified tRNA ${ }^{\text {Asp }}$ and Dnmt2 in the binary complex and subsequent MS analysis. However, we failed to identify a crosslink that involved either the 7-deaza-guanine or the cyclopentene-diol of the queuine base leaving the question of a direct interaction with the enzyme unanswered.

All three explanations of how queuosine might impact Dnmt2 activity on a molecular level are biochemically justifiable. Most convincing is the more general explanation that Q34 triggers Dnmt2 activity through optimized orientation of the components that are involved in the reaction. This is supported by the close proximity of Q34 to the co-substrate and the active site in the complex model. First insights into the role of queuine could be provided by determination of the $k_{\text {on }}$ and $k_{\text {off }}$ values of tRNA binding, that might very well change upon queuine modification despite the macroscopic binding constant is unchanged.

\subsection{DISCUSSION OF A DNMT2 WORKING MODEL}

In this paragraph, a model of a Dnmt2 mediated methyltransfer from the methyldonor SAM to the $\mathrm{C} 38$ of a cognate tRNA is presented. The model is focused on the structural and biochemical observations presented within this thesis for the $S$. pombe system including data reported by other groups.

In contrast to Dnmt1 and 3, which target DNA and therefore are localized in the nucleus, the Dnmt2 enzymatic reaction is likely to occur in the cytoplasm, as the human Dnmt2 enzyme has been shown to localize in this cellular compartment (64). From the docked model of the Dnmt2 substrate complex it can be concluded that binding of the methyldonor SAM to the Dnmt2 enzyme must occur prior to binding of the tRNA substrate, which otherwise would obstruct the SAM binding pocket. Thus, SAM binding likely is the first step in the enzymatic turnover. Interestingly, no Dnmt 2 crystals of a quality suitable for data collection could be observed in absence of the demethylated reaction product SAH and all structures reported previously as well as the herein reported structure of the $S$. pombe Dnmt2 were solved from crystals grown in presence of SAH. This raises the question how SAH contributes to Dnmt 2 crystallization. Given the high flexibility that is observed for the active site loop, SAH binding might foster conformational rigidity at the beginning of this loop and other regions that are involved in SAH 
coordination reducing Dnmt2 flexibility and aiding crystallization. As the second step, the binding of the tRNA substrate is likely to occur which seems to induce conformational changes on the protein and possibly also the RNA side. A striking conformational change has to involve the active site loop, as is was shown for the $S$. pombe system that this loop is in direct contact with RNA substrate. This active site loop is likely folds down onto the nucleotides upon binding of the tRNA and adopting a "closed" conformational state. It has previously been shown for Dnmt1 that methyltransfer requires a flip out of the respective cytosine base into the catalytic pocket to undergo methylation. In this Dnmt1 case a concerted action replaces the target base by a protein residue in the DNA double helix and the cytosine is flipped into the catalytic pocket. Despite the different substrate specificity of Dnmt2, the proven conservation of the catalytic mechanism throughout the Dnmt family, argues that such a base flip out may also occur with respect to this enzyme. This is further supported by the reported tRNA ${ }^{\text {Asp }}$ structure from yeast, in which the C38 base is not flipped out but is engaged in base pairing. Such a flip out could be promoted by residues of the active site loop. A similar action has been observed for the structurally similar HhaI DNA methyltransferase, which was crystallized with the target base flipped out into the active site. In this crystal structure this base is substituted by a residue of a loop that is adjacent to the active site.

The catalytic mechanism of Dnmt 2 has been described for the human enzyme before $(53,183)$. All relevant residues are also conserved in $S$. pombe as well is the structure arguing for a conserved mechanism from human to yeast. In the crystal structure of $S$. pombe Dnmt2 the catalytically relevant Glu121 is positioned towards the catalytic center. In contrast, the catalytic residue Cys81 does not face the active site but rather is turned into the inside of the protein indicating that the catalytically active conformation of Dnmt 2 is fully established only after the substrate is bound. With all residues in place, Cys81 can the nucleophilically attack the cysteine's C4 atom and form the covalent reaction intermediate. Transfer of the methylgroup would then be further promoted by the electron drawing coordination of the base by Glu121 fulfilling the conserved Dnmt reaction mechanism. The reaction product is finally formed through basic attack on the $\mathrm{C} 5$ hydrogen atom resulting in a break of the covalent link between the protein and the RNA that is now free to dissociate from the enzyme. In a last step the SAH must be released, leaving the binding site competent for the next SAM molecule and the turnover can start again. 


\section{CHAPTER 5: SYNOPSIS AND OUTLOOK}

The recently reported interplay of the tRNA modifications Q34 and $\mathrm{m}^{5} \mathrm{C} 38$ in S. pombe (65) is one of the first examples that distinct modifications on the same tRNA molecule can influence their abundance in a downstream manner. However, before the start of this thesis the structure of the enzyme, which incorporates queuine, was not known and the question how Q34 modification affects Dnmt2 on a molecular level and whether other modifications are required was unanswered.

For the structure determination of the human TGT enzyme, the experimental design focused on determination of the functional heterodimer. However, possibly aided by the crystallization condition, only the QTRT1 subunit crystallized. The solution of this structure resulted in the first reported structure of a eukaryotic TGT catalytic subunit and soaking with queuine did not only provide novel insights into the subunit architecture but also in binding of the modified base by QTRT1.

To date the crystal structures of both eukaryotic TGT subunits as well as enzyme kinetics have been reported. Future structural work on the human TGT should focus on structure determination of the functional heterodimer, as the quaternary assembly of this enzyme is still unclear. The underlying experimental design should account for the herein presented observation that the presence of chaotropic salts may promote destabilization of subunit interaction. Beside crystallization of the human TGT heterodimer, purification methods for all components of a tRNA substrate complex have been established within this thesis, supporting crystallization of the tRNA substrate complex. The resulting structure likely could provide long awaited insights into the enigmatic role of the QTRT2 subunit, that was proven to be of essence to the functionality of the enzyme but harbors no functional active site itself. Another interesting topic is the apparent dependence of TGT activity on phosphorylation by PKC. In vitro phosphorylation of the recombinantly expressed and purified heterodimer with subsequent MS analysis could resolve which TGT residue(s) are phosphorylated and crystallization could provide structural insights into conformational changes upon modification that may explain the importance of phosphorylation for TGT activity. 
Beside further investigation of the human TGT enzyme, the heart of this thesis is the structural and biochemical investigation of queuine mediated modulation of Dnmt2 activity. It was shown that queuine alone is capable to promote Dnmt2 activity in structural context of tRNA. Furthermore, a model was generated by computational docking of a tRNA to the newly reported S. pombe Dnmt2 crystal structure. Additional information on the structure of the ternary complex was obtained from cross-linking experiments and allowed to validate the docking model. The model furthermore suggests that queuine at position 34 of the tRNA substrate does trigger Dnmt2 activity through optimized positioning of relevant components rather than being recognized by a pocket on the enzyme surface.

An important element of this thesis is the model of the Dnmt2 substrate complex which was obtained from computational docking of the tRNA to the complex provides new valuable insights how Dnmt2 might bind its tRNA substrate and how queuine might alter Dnmt2 activity. It also promotes a deeper understanding regarding the mode of action of the enzyme and together with cross-linking data argue for an important role of the enzyme's active site loop. Despite the reported complex model is the most advanced as of today, docking of ridged structures has the previously described disadvantages. Therefore, a crystal structure of the Dnmt2 substrate complex is still of imminent importance for the understanding of the Dnmt2 functional mechanism.

Within the experimental work underlying this thesis, a substantial effort was undertaken to obtain the crystal structure of the Dnmt2 enzyme in complex with a substrate tRNA. Despite optimization of crystallization conditions favoring complex formation, crystals obtained from crystallization trials were either of insufficient quality for structure determination, or did contain either the protein or the tRNA, but not the ternary complex. Most promising, was the combination of the E. histolytica Dnmt2 with the human tRNA ${ }^{\text {Asp }}$, which yielded a single crystal that allowed collection of synchrotron datasets up to $3.1 \AA$ A resolution. Processing of this data identified the crystal to belong to the space group P321, which has not been reported for a Dnmt 2 enzyme or tRNA alone. The cell constants, furthermore, proved that both components of the complex could be encompassed in the unit cell of the crystal. Despite extensive data processing and application of numerous search models, the structure could not be solved by molecular replacement. This promising candidate should be in the scope of future work, which should focus on optimization of this crystallization condition to improve crystal quality and employ the use of anomalous scatterers to solve the structure of the complex. Most interesting will be the structure obtained from the use of a Q34 modified tRNA, which could in comparison 
to the unmodified tRNA complex answer the question how queuine modulates Dnmt2 activity on a structural level. Furthermore, queuine dependency of methylation by Dnmt2, although suggested, has not been reported in other organisms than $S$. pombe. The herein reported assay that allows to incorporate queuine into tRNA can serve as a basis for an investigation of Q34 dependency of Dnmt2 for the E. histolytica and the human enzyme. 


\section{CHAPTER 6: REFERENCES}

1. R. Dahm, Discovering DNA: Friedrich Miescher and the early years of nucleic acid research. Hum. Genet. 122, 565-581 (2008).

2. J. D. Watson, F. H. C. Crick, Molecular Structure of Nucleic Acids: A Structure for Deoxyribose Nucleic Acid. Nature. 171, 737-738 (1953).

3. J. D. Watson, F. H. C. Crick, Genetical Implications of the Structure of Deoxyribonucleic Acid. Nature. 171, 964-967 (1953).

4. T. R. Cech, The efficiency and versatility of catalytic RNA: implications for an RNA world. Gene. 135, 33-36 (1993).

5. S. Altman, Enzymatic cleavage of RNA by RNA. Biosci. Rep. 10, 317-337 (1990).

6. R. Lührmann, B. Kastner, M. Bach, Structure of spliceosomal snRNPs and their role in pre-mRNA splicing. Biochim. Biophys. Acta BBA - Gene Struct. Expr. 1087, 265-292 (1990).

7. T. Kiss, Small nucleolar RNA-guided post-transcriptional modification of cellular RNAs. EMBO J. 20, 3617-3622 (2001).

8. L. He, G. J. Hannon, MicroRNAs: small RNAs with a big role in gene regulation. Nat. Rev. Genet. 5, 522-531 (2004).

9. D. Voet, J. Voet, C. Pratt, Fundamentals Of Biochemistry: Life At The Molecular Level. Fundam. Biochem. Life Mol. Level (2016) (available at https://works.swarthmore.edu/facchemistry/105).

10. Lodish, H. F., Darnell, J., Molecular Cell Biology (ed. 3, 1995).

11. P. P. Chan, T. M. Lowe, GtRNAdb 2.0: an expanded database of transfer RNA genes identified in complete and draft genomes. Nucleic Acids Res. 44, D184-D189 (2016).

12. Y. Tomari, T. Suzuki, T. Ueda, tRNA Recognition by CCA-adding enzyme. Nucleic Acids Symp. Ser. 2, 77-78 (2002).

13. M. P. Deutscher, Rat Liver Glutamyl Ribonucleic Acid Synthetase I. PURIFICATION AND EVIDENCE FOR SEPARATE ENZYMES FOR GLUTAMIC ACID AND GLUTAMINE. J. Biol. Chem. 242, 1123-1131 (1967).

14. H. Betat, C. Rammelt, M. Mörl, tRNA nucleotidyltransferases: ancient catalysts with an unusual mechanism of polymerization. Cell. Mol. Life Sci. CMLS. 67, 1447-1463 (2010).

15. M. Sprinzl, C. Horn, M. Brown, A. Ioudovitch, S. Steinberg, Compilation of tRNA sequences and sequences of tRNA genes. Nucleic Acids Res. 26, 148-153 (1998). 
16. R. Green, H. F. Noller, Ribosomes and translation. Annu. Rev. Biochem. 66, 679-716 (1997).

17. M. Simonović, T. A. Steitz, Peptidyl-CCA deacylation on the ribosome promoted by induced fit and the O3'-hydroxyl group of A76 of the unacylated A-site tRNA. RNA N.Y. N. 14, 2372-2378 (2008).

18. M. Messmer et al., Tertiary network in mammalian mitochondrial tRNAAsp revealed by solution probing and phylogeny. Nucleic Acids Res. 37, 6881-6895 (2009).

19. K. Wakita et al., Higher-order structure of bovine mitochondrial tRNA(Phe) lacking the "conserved" GG and T psi CG sequences as inferred by enzymatic and chemical probing. Nucleic Acids Res. 22, 347-353 (1994).

20. D. R. Wolstenholme, J. L. Macfarlane, R. Okimoto, D. O. Clary, J. A. Wahleithner, Bizarre tRNAs inferred from DNA sequences of mitochondrial genomes of nematode worms. Proc. Natl. Acad. Sci. U. S. A. 84, 1324-1328 (1987).

21. F. Jühling, J. Pütz, C. Florentz, P. F. Stadler, Armless mitochondrial tRNAs in Enoplea (Nematoda). RNA Biol. 9, 1161-1166 (2012).

22. A. D. Wolfson, A. M. Khvorova, C. Sauter, C. Florentz, R. Giegé, Mimics of yeast tRNAAsp and their recognition by aspartyl-tRNA synthetase. Biochemistry. 38, 1192611932 (1999).

23. R. Giegé et al., Structure of transfer RNAs: similarity and variability. Wiley Interdiscip. Rev. RNA. 3, 37-61 (2012).

24. L. H. Schulman, H. Pelka, Anticodon switching changes the identity of methionine and valine transfer RNAs. Science. 242, 765-768 (1988).

25. J. Normanly, J. Abelson, tRNA identity. Annu. Rev. Biochem. 58, 1029-1049 (1989).

26. M. A. Rould, J. J. Perona, D. Söll, T. A. Steitz, Structure of E. coli glutaminyl-tRNA synthetase complexed with tRNA(Gln) and ATP at 2.8 A resolution. Science. 246, 1135 1142 (1989).

27. M. Ruff et al., Class II aminoacyl transfer RNA synthetases: crystal structure of yeast aspartyl-tRNA synthetase complexed with tRNA(Asp). Science. 252, 1682-1689 (1991).

28. T. Carell et al., Structure and Function of Noncanonical Nucleobases. Angew. Chem. Int. Ed. 51, 7110-7131 (2012).

29. P. Boccaletto et al., MODOMICS: a database of RNA modification pathways. 2017 update. Nucleic Acids Res. 46, D303-D307 (2018).

30. W. A. Cantara et al., The RNA Modification Database, RNAMDB: 2011 update. Nucleic Acids Res. 39, D195-201 (2011).

31. C. Lorenz, C. E. Lünse, M. Mörl, tRNA Modifications: Impact on Structure and Thermal Adaptation. Biomolecules. 7, 35 (2017). 
32. M. A. Machnicka, A. Olchowik, H. Grosjean, J. M. Bujnicki, Distribution and frequencies of post-transcriptional modifications in tRNAs. RNA Biol. 11, 1619-1629 (2014).

33. F. Harada, S. Nishimura, Possible anticodon sequences of tRNA His, tRNA Asm, and tRNA Asp from Escherichia coli B. Universal presence of nucleoside Q in the first postion of the anticondons of these transfer ribonucleic acids. Biochemistry. 11, 301-308 (1972).

34. A. L. Konevega et al., Purine bases at position 37 of tRNA stabilize codon-anticodon interaction in the ribosomal $\mathrm{A}$ site by stacking and $\mathrm{Mg} 2+$-dependent interactions. RNA. 10, 90-101 (2004).

35. M. Helm et al., The presence of modified nucleotides is required for cloverleaf folding of a human mitochondrial tRNA. Nucleic Acids Res. 26, 1636-1643 (1998).

36. J. M. Gregson et al., Structure of the archaeal transfer RNA nucleoside G*-15 (2-amino4,7-dihydro- 4-oxo-7-beta-D-ribofuranosyl-1H-pyrrolo[2,3-d]pyrimidine-5-carboximi dam ide (archaeosine)). J. Biol. Chem. 268, 10076-10086 (1993).

37. M. Helm, R. Giegé, C. Florentz, A Watson-Crick Base-Pair-Disrupting Methyl Group (m1A9) Is Sufficient for Cloverleaf Folding of Human Mitochondrial tRNALys. Biochemistry. 38, 13338-13346 (1999).

38. K. R. Noon et al., Influence of temperature on tRNA modification in archaea: Methanococcoides burtonii (optimum growth temperature [Topt], 23 degrees C) and Stetteria hydrogenophila (Topt, 95 degrees C). J. Bacteriol. 185, 5483-5490 (2003).

39. J. A. Kowalak, J. J. Dalluge, J. A. McCloskey, K. O. Stetter, The role of posttranscriptional modification in stabilization of transfer RNA from hyperthermophiles. Biochemistry. 33, 7869-7876 (1994).

40. P. C. Durant, D. R. Davis, Stabilization of the anticodon stem-loop of tRNALys, 3 by an A+-C base-pair and by pseudouridine11Edited by I. Tinoco. J. Mol. Biol. 285, 115-131 (1999).

41. R. Oliva, A. Tramontano, L. Cavallo, Mg2+ binding and archaeosine modification stabilize the G15-C48 Levitt base pair in tRNAs. RNA. 13, 1427-1436 (2007).

42. P. F. Agris, F. A. P. Vendeix, W. D. Graham, tRNA's Wobble Decoding of the Genome: 40 Years of Modification. J. Mol. Biol. 366, 1-13 (2007).

43. E. M. Gustilo, F. A. Vendeix, P. F. Agris, tRNA's modifications bring order to gene expression. Curr. Opin. Microbiol. 11, 134-140 (2008).

44. P. F. Agris, Wobble position modified nucleosides evolved to select transfer RNA codon recognition: A modified-wobble hypothesis. Biochimie. 73, 1345-1349 (1991).

45. W. C. Clark, M. E. Evans, D. Dominissini, G. Zheng, T. Pan, tRNA base methylation identification and quantification via high-throughput sequencing. RNA. 22, 1771-1784 (2016). 
46. C. T. Y. Chan et al., A Quantitative Systems Approach Reveals Dynamic Control of tRNA Modifications during Cellular Stress. PLOS Genet. 6, e1001247 (2010).

47. F. Lyko, The DNA methyltransferase family: a versatile toolkit for epigenetic regulation. Nat. Rev. Genet. 19, 81-92 (2018).

48. M. A. Sánchez-Romero, I. Cota, J. Casadesús, DNA methylation in bacteria: from the methyl group to the methylome. Curr. Opin. Microbiol. 25, 9-16 (2015).

49. M. J. Koziol et al., Identification of methylated deoxyadenosines in vertebrates reveals diversity in DNA modifications. Nat. Struct. Mol. Biol. 23, 24-30 (2016).

50. J. Liu et al., Abundant DNA 6mA methylation during early embryogenesis of zebrafish and pig. Nat. Commun. 7, 13052 (2016).

51. A. Zemach, I. E. McDaniel, P. Silva, D. Zilberman, Genome-Wide Evolutionary Analysis of Eukaryotic DNA Methylation. Science. 328, 916-919 (2010).

52. S. Feng et al., Conservation and divergence of methylation patterning in plants and animals. Proc. Natl. Acad. Sci. 107, 8689-8694 (2010).

53. A. Jeltsch, W. Nellen, F. Lyko, Two substrates are better than one: dual specificities for Dnmt2 methyltransferases. Trends Biochem. Sci. 31, 306-308 (2006).

54. G. Raddatz et al., Dnmt2-dependent methylomes lack defined DNA methylation patterns. Proc. Natl. Acad. Sci. 110, 8627-8631 (2013).

55. C. V. Patel, K. P. Gopinathan, Determination of trace amounts of 5-methylcytosine in DNA by reverse-phase high-performance liquid chromatography. Anal. Biochem. 164, 164-169 (1987).

56. F. Capuano, M. Mülleder, R. Kok, H. J. Blom, M. Ralser, Cytosine DNA Methylation Is Found in Drosophila melanogaster but Absent in Saccharomyces cerevisiae, Schizosaccharomyces pombe, and Other Yeast Species. Anal. Chem. 86, 3697-3702 (2014).

57. S. Tweedie et al., Vestiges of a DNA methylation system in Drosophila melanogaster? Nat. Genet. 23, 389-390 (1999).

58. F. Lyko et al., Mammalian (cytosine-5) methyltransferases cause genomic DNA methylation and lethality in Drosophila. Nat. Genet. 23, 363-366 (1999).

59. V. Krauss, G. Reuter, in Progress in Molecular Biology and Translational Science, X. Cheng, R. M. Blumenthal, Eds. (Academic Press, 2011; http://www.sciencedirect.com/science/article/pii/B9780123876850000032), vol. 101 of Modifications of Nuclear DNA and its Regulatory Proteins, pp. 177-191.

60. A. Salzberg, O. Fisher, R. Siman-Tov, S. Ankri, Identification of methylated sequences in genomic DNA of adult Drosophila melanogaster. Biochem. Biophys. Res. Commun. 322, 465-469 (2004). 
61. N. D. Singh, P. F. Arndt, D. A. Petrov, Genomic Heterogeneity of Background Substitutional Patterns in Drosophila melanogaster. Genetics. 169, 709-722 (2005).

62. S. Phalke et al., Retrotransposon silencing and telomere integrity in somatic cells of Drosophila depends on the cytosine-5 methyltransferase DNMT2. Nat. Genet. 41, 696702 (2009).

63. D. Ferres-Marco et al., Epigenetic silencers and Notch collaborate to promote malignant tumours by $R b$ silencing. Nature. 439, 430-436 (2006).

64. M. G. Goll et al., Methylation of tRNAAsp by the DNA Methyltransferase Homolog Dnmt2. Science. 311, 395-398 (2006).

65. M. Müller et al., Dynamic modulation of Dnmt2-dependent tRNA methylation by the micronutrient queuine. Nucleic Acids Res. 43, 10952-10962 (2015).

66. R. Shanmugam et al., The Dnmt2 RNA methyltransferase homolog of Geobacter sulfurreducens specifically methylates tRNA-Glu. Nucleic Acids Res. 42, 6487-6496 (2014).

67. M. Becker et al., Pmt1, a Dnmt2 homolog in Schizosaccharomyces pombe, mediates tRNA methylation in response to nutrient signaling. Nucleic Acids Res. 40, 11648-11658 (2012).

68. A. Jeltsch et al., Mechanism and biological role of Dnmt2 in Nucleic Acid Methylation. RNA Biol. 14, 1108-1123 (2017).

69. T. P. Jurkowski, R. Shanmugam, M. Helm, A. Jeltsch, Mapping the tRNA Binding Site on the Surface of Human DNMT2 Methyltransferase. Biochemistry. 51, 4438-4444 (2012).

70. E. C. Schulz, H. M. Roth, S. Ankri, R. Ficner, Structure Analysis of Entamoeba histolytica DNMT2 (EhMeth). PLoS ONE. 7, e38728 (2012).

71. S. Kaiser et al., The RNA methyltransferase Dnmt2 methylates DNA in the structural context of a tRNA. RNA Biol. 14, 1241-1251 (2017).

72. A. Dong et al., Structure of human DNMT2, an enigmatic DNA methyltransferase homolog that displays denaturant-resistant binding to DNA. Nucleic Acids Res. 29, 439448 (2001).

73. S. Li et al., Functional and structural characterization of DNMT2 from Spodoptera frugiperda. J. Mol. Cell Biol. 5, 64-66 (2013).

74. S. Klimasauskas, S. Kumar, R. J. Roberts, X. Cheng, Hhal methyltransferase flips its target base out of the DNA helix. Cell. 76, 357-369 (1994).

75. G. C. Vieira et al., Evolution of DNMT2 in drosophilids: Evidence for positive and purifying selection and insights into new protein (pathways) interactions. Genet. Mol. Biol. 41, 215-234 (2018).

76. M. Schaefer, F. Lyko, Solving the Dnmt2 enigma. Chromosoma. 119, 35-40 (2009). 
77. M. Schaefer et al., RNA methylation by Dnmt2 protects transfer RNAs against stressinduced cleavage. Genes Dev. 24, 1590-1595 (2010).

78. Z. Durdevic, M. B. Mobin, K. Hanna, F. Lyko, M. Schaefer, The RNA Methyltransferase Dnmt2 Is Required for Efficient Dicer-2-Dependent siRNA Pathway Activity in Drosophila. Cell Rep. 4, 931-937 (2013).

79. P. Ivanov, M. M. Emara, J. Villen, S. P. Gygi, P. Anderson, Angiogenin-Induced tRNA Fragments Inhibit Translation Initiation. Mol. Cell. 43, 613-623 (2011).

80. R. Shanmugam et al., Cytosine methylation of tRNA-Asp by DNMT2 has a role in translation of proteins containing poly-Asp sequences. Cell Discov. 1, 15010 (2015).

81. V. Khoddami, B. R. Cairns, Identification of direct targets and modified bases of RNA cytosine methyltransferases. Nat. Biotechnol. 31, 458-464 (2013).

82. B. Brzezicha et al., Identification of human tRNA:m5C methyltransferase catalysing intron-dependent $\mathrm{m} 5 \mathrm{C}$ formation in the first position of the anticodon of the $\$\{$ lhbox \{ pre-tRNA $\}\}_{\{}\{\operatorname{left}(\backslash \text { hbox }\{\text { CAA }\} \backslash \text { right })\}^{\wedge}\{$ ไhbox $\{$ Leu $\}\} \$$. Nucleic Acids Res. 34, 6034-6043 (2006).

83. F. Tuorto et al., RNA cytosine methylation by Dnmt 2 and NSun 2 promotes tRNA stability and protein synthesis. Nat. Struct. Mol. Biol. 19, 900-905 (2012).

84. F. Tuorto et al., The tRNA methyltransferase Dnmt 2 is required for accurate polypeptide synthesis during haematopoiesis. EMBO J. 34, 2350-2362 (2015).

85. Q. Chen et al., Sperm tsRNAs contribute to intergenerational inheritance of an acquired metabolic disorder. Science. 351, 397-400 (2016).

86. Y. Zhang et al., Dnmt2 mediates intergenerational transmission of paternally acquired metabolic disorders through sperm small non-coding RNAs. Nat. Cell Biol. 20, 535-540 (2018).

87. U. Sharma et al., Biogenesis and function of tRNA fragments during sperm maturation and fertilization in mammals. Science. 351, 391-396 (2016).

88. H. Peng et al., A novel class of tRNA-derived small RNAs extremely enriched in mature mouse sperm. Cell Res. 22, 1609-1612 (2012).

89. A. B. Rodgers, C. P. Morgan, N. A. Leu, T. L. Bale, Transgenerational epigenetic programming via sperm microRNA recapitulates effects of paternal stress. Proc. Natl. Acad. Sci. U. S. A. 112, 13699-13704 (2015).

90. K. Gapp et al., Implication of sperm RNAs in transgenerational inheritance of the effects of early trauma in mice. Nat. Neurosci. 17, 667-669 (2014).

91. H. M. Goodman, J. Abelson, A. Landy, S. Brenner, J. D. Smith, Amber Suppression: a Nucleotide Change in the Anticodon of a Tyrosine Transfer RNA. Nature. 217, 10191024 (1968). 
92. B. P. Doctor, J. E. Loebel, M. A. Sodd, D. B. Winter, Nucleotide Sequence of Escherichia coli Tyrosine Transfer Ribonucleic Acid. Science. 163, 693-695 (1969).

93. M. Vinayak, C. Pathak, Queuosine modification of tRNA: its divergent role in cellular machinery. Biosci. Rep. 30, 135-148 (2010).

94. S. Yokoyama et al., Three-dimensional structure of hyper-modified nucleoside Q located in the wobbling position of tRNA. Nature. 282, 107-109 (1979).

95. H. Kasai et al., Structure of the modified nucleoside Q isolated from Escherichia coli transfer ribonucleic acid. 7-(4,5-cis-Dihydroxy-1-cyclopenten-3-ylaminomethyl)-7deazaguanosine. Biochemistry. 14, 4198-4208 (1975).

96. Y. Kuchino, H. Kasai, K. Nihei, S. Nishimura, Biosynthesis of the modified nucleoside Q in transfer RNA. Nucleic Acids Res. 3, 393-398 (1976).

97. N. Okada et al., Novel mechanism of post-transcriptional modification of tRNA. Insertion of bases of Q precursors into tRNA by a specific tRNA transglycosylase reaction. J. Biol. Chem. 254, 3067-3073 (1979).

98. Katze, B. Basile, J. A. McCloskey, Queuine, a modified base incorporated posttranscriptionally into eukaryotic transfer RNA: wide distribution in nature. Science. 216, 55-56 (1982).

99. G. Phillips et al., Biosynthesis of 7-Deazaguanosine-Modified tRNA Nucleosides: a New Role for GTP Cyclohydrolase I. J. Bacteriol. 190, 7876-7884 (2008).

100. G. Katzenmeier, C. Schmid, A. Bacher, Cloning and expression of the putative gene coding for GTP cyclohydrolase I from Escherichia coli. FEMS Microbiol. Lett. 66, 231234.

101. H. Nar et al., Active site topology and reaction mechanism of GTP cyclohydrolase I. Proc. Natl. Acad. Sci. 92, 12120-12125 (1995).

102. J. J. Yim, G. M. Brown, Characteristics of guanosine triphosphate cyclohydrolase I purified from Escherichia coli. J. Biol. Chem. 251, 5087-5094 (1976).

103. R. M. McCarty, Á. Somogyi, V. Bandarian, Escherichia coli QueD Is a 6-Carboxy5,6,7,8-tetrahydropterin Synthase. Biochemistry. 48, 2301-2303 (2009).

104. Z. D. Miles, S. A. Roberts, R. M. McCarty, V. Bandarian, Biochemical and Structural Studies of 6-Carboxy-5,6,7,8-tetrahydropterin Synthase Reveal the Molecular Basis of Catalytic Promiscuity within the Tunnel-fold Superfamily. J. Biol. Chem. 289, 2364123652 (2014).

105. D. P. Dowling et al., Radical SAM enzyme QueE defines a new minimal core fold and metal-dependent mechanism. Nat. Chem. Biol. 10, 106-112 (2014).

106. R. M. McCarty, Á. Somogyi, G. Lin, N. E. Jacobsen, V. Bandarian, The deazapurine biosynthetic pathway revealed: In vitro enzymatic synthesis of preQ0 from guanosine-5'triphosphate in four steps. Biochemistry. 48, 3847-3852 (2009). 
107. R. Gaur, U. Varshney, Genetic Analysis Identifies a Function for the queC (ybaX) Gene Product at an Initial Step in the Queuosine Biosynthetic Pathway in Escherichia coli. J. Bacteriol. 187, 6893-6901 (2005).

108. N. Cicmil, R. H. Huang, Crystal structure of QueC from Bacillus subtilis: An enzyme involved in preQ1 biosynthesis. Proteins Struct. Funct. Bioinforma. 72, 1084-1088.

109. B. W. K. Lee, S. G. Van Lanen, D. Iwata-Reuyl, Mechanistic Studies of Bacillus subtilis QueF, the Nitrile Oxidoreductase Involved in Queuosine Biosynthesis. Biochemistry. 46, 12844-12854 (2007).

110. V. M. Chikwana et al., Structural Basis of Biological Nitrile Reduction. J. Biol. Chem. 287, 30560-30570 (2012).

111. R. K. Slany, M. Bösl, H. Kersten, Transfer and isomerization of the ribose moiety of AdoMet during the biosynthesis of queuosine tRNAs, a new unique reaction catalyzed by the QueA protein from Escherichia coli. Biochimie. 76, 389-393 (1994).

112. C. Grimm et al., Crystal structure of Bacillus subtilis S-adenosylmethionine:tRNA ribosyltransferase-isomerase. Biochem. Biophys. Res. Commun. 351, 695-701 (2006).

113. S. G. Van Lanen, D. Iwata-Reuyl, Kinetic Mechanism of the tRNA-Modifying Enzyme S-Adenosylmethionine:tRNA Ribosyltransferase-Isomerase (QueA). Biochemistry. 42, 5312-5320 (2003).

114. Z. D. Miles, W. K. Myers, W. M. Kincannon, R. D. Britt, V. Bandarian, Biochemical and Spectroscopic Studies of Epoxyqueuosine Reductase: A Novel Iron-Sulfur Clusterand Cobalamin-Containing Protein Involved in the Biosynthesis of Queuosine. Biochemistry. 54, 4927-4935 (2015).

115. K. A. P. Payne et al., Epoxyqueuosine Reductase Structure Suggests a Mechanism for Cobalamin-dependent tRNA Modification. J. Biol. Chem. 290, 27572-27581 (2015).

116. D. P. Dowling et al., Molecular basis of cobalamin-dependent RNA modification. Nucleic Acids Res. 44, 9965-9976 (2016).

117. D. Iwata-Reuyl, in DNA and RNA Modifi cation Enzymes: Structure, Mechanism, Function and Evolution (Landes Bioscience, Austin, Texas).

118. R. Zallot, Y. Yuan, V. de Crécy-Lagard, The Escherichia coli COG1738 Member YhhQ Is Involved in 7-Cyanodeazaguanine (preQ0) Transport. Biomolecules. 7, 12 (2017).

119. G. Ott, H. Kersten, S. Nishimura, Dictyostelium discoideum: a useful model system to evaluate the function of queuine and of the Q-family of tRNAs. FEBS Lett. 146, 311-314.

120. G. M. Kirtland et al., Novel salvage of queuine from queuosine and absence of queuine synthesis in Chlorella pyrenoidosa and Chlamydomonas reinhardtii. J. Bacteriol. 170, 5633-5641 (1988).

121. T. J. Siard, K. B. Jacobson, W. R. Farkas, Queuine metabolism and cadmium toxicity in Drosophila melanogaster. BioFactors Oxf. Engl. 3, 41-47 (1991). 
122. R. Gaur, G. R. Björk, S. Tuck, U. Varshney, Diet-dependent depletion of queuosine in tRNAs in Caenorhabditis elegans does not lead to a developmental block. J. Biosci. 32, 747-754 (2007).

123. J. P. Reyniers, J. R. Pleasants, B. S. Wostmann, J. R. Katze, W. R. Farkas, Administration of exogenous queuine is essential for the biosynthesis of the queuosinecontaining transfer RNAs in the mouse. J. Biol. Chem. 256, 11591-11594 (1981).

124. C. Fergus, D. Barnes, M. A. Alqasem, V. P. Kelly, The Queuine Micronutrient: Charting a Course from Microbe to Man. Nutrients. 7, 2897-2929 (2015).

125. N. Okada, S. Nishimura, Isolation and characterization of a guanine insertion enzyme, a specific tRNA transglycosylase, from Escherichia coli. J. Biol. Chem. 254, 3061-3066 (1979).

126. Y.-C. Chen et al., Evolution of eukaryal tRNA-guanine transglycosylase: insight gained from the heterocyclic substrate recognition by the wild-type and mutant human and Escherichia coli tRNA-guanine transglycosylases. Nucleic Acids Res. 39, 2834-2844 (2011).

127. J. R. Katze, Queuosine metabolism: possible relation to B-cell activation by C8 derivatives of guanosine. Proc. Soc. Exp. Biol. Med. Soc. Exp. Biol. Med. N. Y. N. 179, 492-496 (1985).

128. J. R. Katze, W. T. Beck, C. S. Cheng, J. A. McCloskey, Why is tumor tRNA hypomodified with respect to Q nucleoside? Recent Results Cancer Res. Fortschritte Krebsforsch. Progres Dans Rech. Sur Cancer. 84, 146-159 (1983).

129. U. Gündüz, J. R. Katze, Queuine salvage in mammalian cells. Evidence that queuine is generated from queuosine 5'-phosphate. J. Biol. Chem. 259, 1110-1113 (1984).

130. W. R. Farkas, K. B. Jacobson, J. R. Katze, Substrate and inhibitor specificity of tRNAguanine ribosyltransferase. Biochim. Biophys. Acta BBA - Gene Struct. Expr. 781, 64-75 (1984).

131. R. C. Morris, M. C. Galicia, K. L. Clase, M. S. Elliott, Determination of Queuosine Modification System Deficiencies in Cultured Human Cells. Mol. Genet. Metab. 68, 5667 (1999).

132. M. S. Elliott, R. W. Trewyn, J. R. Katze, Inhibition of Queuine Uptake in Cultured Human Fibroblasts by Phorbol-12,13-didecanoate. Cancer Res. 45, 1079-1085 (1985).

133. H. Kasai, Y. Kuchino, K. Nihei, S. Nishimura, Distribution of the modified nucleoside $\mathrm{Q}$ and its derivatives in animal and plant transfer RNA's. Nucleic Acids Res. 2, 19311940 (1975).

134. H. Kasai et al., The structure of $\mathrm{Q}^{*}$ nucleoside isolated from rabbit liver transfer ribonucleic acid. J. Am. Chem. Soc. 98, 5044-5046 (1976).

135. N. Okada, S. Nishimura, Enzymatic synthesis of $Q^{*}$ nucleoside containing mannose in the anticodon of tRNA: isolation of a novel mannosyltransferase from a cell-free extract of rat liver. Nucleic Acids Res. 4, 2931-2937 (1977). 
136. L. L. Lairson, B. Henrissat, G. J. Davies, S. G. Withers, Glycosyltransferases: structures, functions, and mechanisms. Annu. Rev. Biochem. 77, 521-555 (2008).

137. B. Stengl, K. Reuter, G. Klebe, Mechanism and Substrate Specificity of tRNAGuanine Transglycosylases (TGTs): tRNA-Modifying Enzymes from the Three Different Kingdoms of Life Share a Common Catalytic Mechanism. ChemBioChem. 6, 1926-1939 (2005).

138. D. Iwata-Reuyl, Biosynthesis of the 7-deazaguanosine hypermodified nucleosides of transfer RNA. Bioorganic Chem. 31, 24-43 (2003).

139. C. Romier, K. Reuter, D. Suck, R. Ficner, Crystal structure of tRNA-guanine transglycosylase: RNA modification by base exchange. EMBO J. 15, 2850-2857 (1996).

140. R. Ishitani et al., Crystal structure of archaeosine tRNA-guanine transglycosylase. $J$. Mol. Biol. 318, 665-677 (2002).

141. C. Behrens et al., Homodimer Architecture of QTRT2, the Noncatalytic Subunit of the Eukaryotic tRNA-Guanine Transglycosylase. Biochemistry (2018), doi:10.1021/acs.biochem.8b00294.

142. W. Xie, X. Liu, R. H. Huang, Chemical trapping and crystal structure of a catalytic tRNA guanine transglycosylase covalent intermediate. Nat. Struct. Mol. Biol. 10, 781-788 (2003).

143. R. Ishitani et al., Alternative Tertiary Structure of tRNA for Recognition by a Posttranscriptional Modification Enzyme. Cell. 113, 383-394 (2003).

144. S. Nakanishi et al., A UGU sequence in the anticodon loop is a minimum requirement for recognition by Escherichia coli tRNA-guanine transglycosylase. J. Biol. Chem. 269, 32221-32225 (1994).

145. A. W. Curnow, G. A. Garcia, tRNA-guanine Transglycosylase from Escherichia coli MINIMAL tRNA STRUCTURE AND SEQUENCE REQUIREMENTS FOR RECOGNITION. J. Biol. Chem. 270, 17264-17267 (1995).

146. J. Song, M. Teplova, S. Ishibe-Murakami, D. J. Patel, Structure-Based Mechanistic Insights into DNMT1-Mediated Maintenance DNA Methylation. Science. 335, 709-712 (2012).

147. A. Guelorget, B. Golinelli-Pimpaneau, Mechanism-Based Strategies for Trapping and Crystallizing Complexes of RNA-Modifying Enzymes. Structure. 19, 282-291 (2011).

148. C. Romier, J. E. Meyer, D. Suck, Slight sequence variations of a common fold explain the substrate specificities of tRNA-guanine transglycosylases from the three kingdoms. FEBS Lett. 416, 93-98 (1997).

149. Y. Bai, D. T. Fox, J. A. Lacy, S. G. V. Lanen, D. Iwata-Reuyl, Hypermodification of tRNA in Thermophilic Archaea CLONING, OVEREXPRESSION, AND CHARACTERIZATION OF tRNA-GUANINE TRANSGLYCOSYLASE FROM METHANOCOCCUS JANNASCHII. J. Biol. Chem. 275, 28731-28738 (2000). 
150. M. Watanabe et al., Biosynthesis of Archaeosine, a Novel Derivative of 7Deazaguanosine Specific to Archaeal tRNA, Proceeds via a Pathway Involving Base Replacement on the tRNA Polynucleotide Chain. J. Biol. Chem. 272, 20146-20151 (1997).

151. G. Phillips et al., Discovery and Characterization of an Amidinotransferase Involved in the Modification of Archaeal tRNA . J. Biol. Chem. 285, 12706-12713 (2010).

152. H. Shi, P. B. Moore, The crystal structure of yeast phenylalanine tRNA at 1.93 A resolution: a classic structure revisited. $R N A$. 6, 1091-1105 (2000).

153. R. Zallot et al., Plant, Animal, and Fungal Micronutrient Queuosine Is Salvaged by Members of the DUF2419 Protein Family. ACS Chem. Biol. 9, 1812-1825 (2014).

154. Y. C. Chen, V. P. Kelly, S. V. Stachura, G. A. Garcia, Characterization of the human tRNA-guanine transglycosylase: Confirmation of the heterodimeric subunit structure. RNA. 16, 958-968 (2010).

155. S. G. Ozanick, J. M. Bujnicki, D. S. Sem, J. T. Anderson, Conserved amino acids in each subunit of the heteroligomeric tRNA m 1 A58 Mtase from Saccharomyces cerevisiae contribute to tRNA binding. Nucleic Acids Res. 35, 6808-6819 (2007).

156. P. Barraud et al., Crystal Structure of Thermus thermophilus tRNA m1A58 Methyltransferase and Biophysical Characterization of Its Interaction with tRNA. J. Mol. Biol. 377, 535-550 (2008).

157. C. Boland, P. Hayes, I. Santa-Maria, S. Nishimura, V. P. Kelly, Queuosine Formation in Eukaryotic tRNA Occurs via a Mitochondria-localized Heteromeric Transglycosylase. J. Biol. Chem. 284, 18218-18227 (2009).

158. I. Biela et al., Investigation of Specificity Determinants in Bacterial tRNA-Guanine Transglycosylase Reveals Queuine, the Substrate of Its Eucaryotic Counterpart, as Inhibitor. PLoS ONE. 8 (2013), doi:10.1371/journal.pone.0064240.

159. R. C. Morris et al., Activation of transfer RNA-guanine ribosyltransferase by protein kinase C. Nucleic Acids Res. 23, 2492-2498 (1995).

160. S. Nachtergaele, C. He, The emerging biology of RNA post-transcriptional modifications. RNA Biol. 14, 156-163 (2017).

161. A. E. Ehrenhofer-Murray, Cross-Talk between Dnmt2-Dependent tRNA Methylation and Queuosine Modification. Biomolecules. 7, 14 (2017).

162. M. S. Elliott, J. R. Katze, R. W. Trewyn, Relationship between a Tumor Promoterinduced Decrease in Queuine Modification of Transfer RNA in Normal Human Cells and the Expression of an Altered Cell Phenotype. Cancer Res. 44, 3215-3219 (1984).

163. R. W. Trewyn, H. B. Gatz, Altered growth properties of normal human cells induced by phorbol 12,13-didecanoate. In Vitro. 20, 409-415 (1984). 
164. M. S. Elliott, J. R. Katze, Inhibition of queuine uptake in diploid human fibroblasts by phorbol-12,13-didecanoate. Requirement for a factor derived from early passage cells. $J$. Biol. Chem. 261, 13019-13025 (1986).

165. M. S. Elliott, D. L. Crane, Interferon induced inhibition of queuine uptake in cultured human fibroblasts. Biochem. Biophys. Res. Commun. 171, 384-392 (1990).

166. G. Dirheimer, W. Baranowski, G. Keith, Variations in tRNA modifications, particularly of their queuine content in higher eukaryotes. Its relation to malignancy grading. Biochimie. 77, 99-103 (1995).

167. B.-S. Huang, R.-T. Wu, K.-Y. Chien, Relationship of the Queuine Content of Transfer Ribonucleic Acids to Histopathological Grading and Survival in Human Lung Cancer. Cancer Res. 52, 4696-4700 (1992).

168. B. Emmerich et al., Relationship of Queuine-lacking Transfer RNAs to the Grade of Malignancy in Human Leukemias and Lymphomas. Cancer Res. 45, 4308-4314 (1985).

169. U. Aytaç, U. Gündüz, Q-modification of tRNAs in human brain tumors. Cancer Biochem. Biophys. 14, 93-98 (1994).

170. A. Bednářová et al., Lost in Translation: Defects in Transfer RNA Modifications and Neurological Disorders. Front. Mol. Neurosci. 10 (2017), doi:10.3389/fnmol.2017.00135.

171. N. Shindo-Okada, M. Terada, S. Nishimura, Changes in amount of hypo-modified tRNA having guanine in place of queuine during erythroid differentiation of murine erythroleukemia cells. Eur. J. Biochem. 115, 423-428 (1981).

172. G. C. Blobe, L. M. Obeid, Y. A. Hannun, Regulation of protein kinase C and role in cancer biology. Cancer Metastasis Rev. 13, 411-431 (1994).

173. J. R. Katze, W. T. Beck, Administration of queuine to mice relieves modified nucleoside queuosine deficiency in Ehrlich ascites tumor tRNA. Biochem. Biophys. Res. Commun. 96, 313-319 (1980).

174. D. A. Heldman, M. R. Grever, C. E. Speicher, R. W. Trewyn, Urinary excretion of modified nucleosides in chronic myelogenous leukemia. J. Lab. Clin. Med. 101, 783-792 (1983).

175. E. Borek et al., High Turnover Rate of Transfer RNA in Tumor Tissue. Cancer Res. 37, 3362-3366 (1977).

176. S. B. Rothbart, B. D. Strahl, Interpreting the language of histone and DNA modifications. Biochim. Biophys. Acta BBA - Gene Regul. Mech. 1839, 627-643 (2014).

177. B. J. Wilkins et al., A Cascade of Histone Modifications Induces Chromatin Condensation in Mitosis. Science. 343, 77-80 (2014).

178. L. Zhao et al., 'O-GlcNAc Code' Mediated Biological Functions of Downstream Proteins. Molecules. 23, 1967 (2018). 
179. H. Zhou et al., Toward a comprehensive characterization of a human cancer cell phosphoproteome. J. Proteome Res. 12, 260-271 (2013).

180. N. Blom, S. Gammeltoft, S. Brunak, Sequence and structure-based prediction of eukaryotic protein phosphorylation sites. J. Mol. Biol. 294, 1351-1362 (1999).

181. L.-Y. Tang et al., The Eukaryotic DNMT2 Genes Encode a New Class of Cytosine-5 DNA Methyltransferases. J. Biol. Chem. 278, 33613-33616 (2003).

182. S. Johannsson et al., Structural insights into the stimulation of S. pombe Dnmt2 catalytic efficiency by the tRNA nucleoside queuosine. Sci. Rep. 8, 8880 (2018).

183. T. P. Jurkowski et al., Human DNMT2 methylates tRNAAsp molecules using a DNA methyltransferase-like catalytic mechanism. RNA. 14, 1663-1670 (2008).

184. A. Guilhot-Gaudeffroy, C. Froidevaux, J. Azé, J. Bernauer, Protein-RNA Complexes and Efficient Automatic Docking: Expanding RosettaDock Possibilities. PLOS ONE. 9, e108928 (2014).

185. F. Jühling et al., tRNAdb 2009: compilation of tRNA sequences and tRNA genes. Nucleic Acids Res. 37, D159-D162 (2009). 


\section{ABBREVIATIONS}

\begin{tabular}{|c|c|}
\hline$-1-$ & double knock out \\
\hline $6 \mathrm{xHis}$ tag & hexa-histidine tag \\
\hline $9 \mathrm{dzG}$ & 9-deaza-guanine \\
\hline A & adenosine \\
\hline $\mathrm{A}_{280}$ & absorption at $280 \mathrm{~nm}$ wavelength \\
\hline aaRS & aminoacyl tRNA synthase \\
\hline $\operatorname{arcTGT}$ & archaebacterial tRNA guanine transglycosylase \\
\hline ATP & adenosine 5'-triphosphate \\
\hline bacTGT & bacterial tRNA guanine transglycosylase \\
\hline BSA & bovine serum albumin \\
\hline $\mathrm{C}$ & cytidine \\
\hline $\mathrm{CpG}$ & cytidine-phosphate-guanosine \\
\hline $\mathrm{CPH}_{4}$ & 6-carboxy-5,6,7,8-tetrahydropterin \\
\hline $\mathrm{CV}$ & column volume \\
\hline $\mathrm{dA}$ & deoxyadenosine \\
\hline $\mathrm{dC}$ & deoxycytidine \\
\hline DESY & Deutsches Elektronen-Syncrotron \\
\hline DFG & deutsche Forschungsgemeinschaft \\
\hline $\mathrm{dG}$ & deoxyguanosine \\
\hline DNA & deoxyribonucleic acid \\
\hline Dnmt & DNA methyltransferase \\
\hline $\mathrm{dT}$ & deoxythymidine \\
\hline DTT & dithiothreitol \\
\hline $\mathrm{E}_{515}$ & extinction coefficient at $515 \mathrm{~nm}$ wavelength \\
\hline EDTA & ethylenediaminetetraacetic acid \\
\hline EMBL & European Molecular Biology Laboratory \\
\hline ESI & electrospray ionization \\
\hline
\end{tabular}




\begin{tabular}{|c|c|}
\hline euTGT & eukaryotic tRNA guanine transglycosylase \\
\hline G & guanosine \\
\hline $\mathrm{G}^{*}$ & archaeosine \\
\hline galQ & galactosyl-queuosine \\
\hline GST & glutathione S-transferase \\
\hline GTP & guanosine 5'-triphosphate \\
\hline $\mathrm{H}_{2} \mathrm{NTP}$ & 7,8-dihydroneopterin triphosphate \\
\hline HEPES & 4-(2-Hydroxyethyl)piperazine-1-ethanesulfonic acid \\
\hline HFD & high-fat diet \\
\hline HFF & human foreskin fibroblasts \\
\hline HPLC & high pressure liquid chromatography \\
\hline HT & HEPES transcription \\
\hline $\mathrm{m}^{1} \mathrm{~A}$ & N1-methyladenosine \\
\hline $\mathrm{m}^{4} \mathrm{C}$ & N4-methylcytosine \\
\hline $\mathrm{m}^{5} \mathrm{C}$ & C5-methylcytosine \\
\hline $\mathrm{m}^{6} \mathrm{~A}$ & N6-methyladenine \\
\hline manQ & mannosyl-queuosine \\
\hline MES & 2-(N-morpholino)ethanesulfonic acid \\
\hline micro RNAs & micro ribonucleic acid \\
\hline MR & molecular replacement \\
\hline mRNA & messenger ribonucleic acid \\
\hline MS & mass spectrometry \\
\hline MSC & multipotent stem cell \\
\hline MWCO & molecular weight cut out \\
\hline $\mathrm{NADPH} / \mathrm{H}^{+}$ & reduced form of nicotinamide adenine dinucleotide phosphate \\
\hline NMR & nuclear magnetic resonance \\
\hline PDB & Protein Data Bank \\
\hline PDD & phorbol-12,13-didecanoate \\
\hline PEG & polyethylene glycol \\
\hline PGA & $\gamma$-polyglutamic acid \\
\hline
\end{tabular}


PKC

Q

$\mathrm{Q}^{*}$

RMSD

RNA

$\mathrm{rQT}_{3}$

SAD

$\mathrm{SAH}$

SAM

SD

SDS-PAGE

siRNA

snoRNA

snRNA

TGT

TLC

Tris

tRNA

tsRNAs

U

UDP

$\mathrm{UV} / \mathrm{Vis}$

WT

$\mathrm{yW}$

$\psi$ protein kinase $\mathrm{C}$

queuosine

modified queuine base/queuosine

root mean square deviation

ribonucleic acid

reduced queuine tritium derivative

single-wavelength anomalous dispersion

S-adenosyl-homocysteine

S-adenosyl-methionine

standard deviation

sodium dodecyl sulfate polyacrylamide gel electrophoresis

small interfering ribonucleic acid

small nucleolar ribonucleic acid

small nuclear ribonucleic acid

tRNA guanine transglycosylase

thin-layer chromatography

tris(hydroxymethyl)aminomethane

transfer ribonucleic acid

tRNA-derived small ribonucleic acid

uridine

uridine diphosphate

ultraviolet and visible fraction of electromagnetic radiation

wild type

wybutosine

pseudouridine 


\section{ACKNOWLEDGEMENTS}

The work underlying this thesis was carried out in the Department for Molecular Structural Biology at the Göttingen Centre for Molecular Biosciences (GZMB). I am deeply grateful for the opportunity to work on this thesis, given to me by my supervisor Prof. Dr. Ralf Ficner who provided a great and productive research environment that made this thesis in its herein described extend possible. I truly appreciated his sincere interest in this project and his outstanding and continuous support. Furthermore, I would like to thank him for the multiple opportunities to present my work to the scientific community at international conferences.

As well, I acknowledge the support of the members of my thesis committee, Prof. Dr. Holger Stark and Prof. Dr. Claudia Höbartner, who showed great interest in my work and supported this thesis with their advice and guidance.

I am grateful to Prof. Dr. Henning Urlaub, Prof. Dr. Kai Tittmann and Prof. Dr. Markus Bohnsack for being part of the extended examination board.

During my thesis I highly appreciated the outstanding support of Dr. Piotr Neumann, who was always available for the discussion on crystallographic matters. I am most thankful for sharing his extraordinary understanding of biomolecular crystallography on numerous occasions during these three years. Also, I kindly like to thank Dr. Achim Dickmanns, who was always helpful with his expertise on laboratory work.

I express my sincere thanks to all members of the MSB department for their friendship and creating a welcoming working atmosphere. Especially I thank Dr. Marcel Tauchert, Johannes Arens, Florian, Hamann and Katharina Blersch for their support and friendship. I also like to thank my students, Alexander Lockhorn and Nora Eulig, for the opportunity of working together and participate in their scientific education.

Furthermore, I like to thank the members of the group of Prof. Dr. Kai Tittman. Especially I appreciated the scientific advice of Fabienne Libuda and Sören Rindfleisch. I like to thank Fabienne Libuda for the scientific discussions and her long-lasting friendship around the globe. I like to send many thanks to Sven Haroth and Hanna Brandt for their friendship and for the outstanding sportive adventures we shared. These made this time truly rememberable. 
This thesis would not have been possible without the continuous support of my parents, Marina Johannsson and Harald Johannsson as well as my grandparents Magrit Salota and Herbert Salota. As of their extraordinary support during my scientific career, I dedicate this thesis to them. 

